\title{
Secondary large-scale index theory and positive scalar curvature
}

\author{
Dissertation \\ zur Erlangung des mathematisch-naturwissenschaftlichen Doktorgrades \\ "Doctor rerum naturalium" \\ der Georg-August-Universität Göttingen \\ im Promotionsprogramm „School of Mathematical Sciences“ \\ der Georg-August University School of Science (GAUSS)
}

vorgelegt von

Rudolf Zeidler

aus Wien

Göttingen, 2016 


\section{Betreungsausschuss}

Erstbetreuer: Prof. Dr. Thomas Schick

Mathematisches Institut, Georg-August-Universität Göttingen

Zweitbetreuer: Prof. Dr. Ralf Meyer

Mathematisches Institut, Georg-August-Universität Göttingen

\section{Mitglieder der Prüfungskommission}

Referent: Prof. Dr. Thomas Schick

Mathematisches Institut, Georg-August-Universität Göttingen

Koreferent: Prof. Dr. Ralf Meyer

Mathematisches Institut, Georg-August-Universität Göttingen

\section{Weitere Mitglieder der Prüfungskommission}

\section{Prof. Dr. Dorothea Bahns}

Mathematisches Institut, Georg-August-Universität Göttingen

Prof. Dr. Karl-Henning Rehren

Institut für Theoretische Physik, Georg-August-Universität Göttingen

Prof. Dr. Max Wardetzky

Institut für Numerische und Angewandte Mathematik,

Georg-August-Universität Göttingen

\section{Prof. Dr. Chenchang Zhu}

Mathematisches Institut, Georg-August-Universität Göttingen

\section{Tag der mündlichen Prüfung}

24.08 .2016 


\begin{abstract}
We develop a theory of secondary invariants associated to complete Riemannian metrics of uniformly positive scalar curvature outside a prescribed subset on a spin manifold. We work in the context of large-scale (or "coarse") index theory. These invariants can be used to distinguish such Riemannian metrics up to concordance relative to the prescribed subset. We exhibit a general external product formula for partial secondary invariants, from which we deduce product formulas for the $\rho$-invariant of a metric with uniformly positive scalar curvature as well as for the coarse index difference of two metrics with uniformly positive scalar curvature.

Our methods yield a new conceptual proof of the secondary partitioned manifold index theorem and a refined version of the delocalized APS-index theorem of PiazzaSchick for the spinor Dirac operator in all dimensions. We establish a partitioned manifold index theorem for the coarse index difference. Moreover, we reprove the existence of a transformation from the positive scalar curvature sequence of Stolz to the analytic surgery sequence of Higson-Roe for real K-theory.

As applications of our theory, we construct several complete metrics of uniformly positive scalar curvature on non-compact spin manifolds which can be distinguished up to concordance relative to certain subsets. Moreover, we establish variants of obstructions to existence and concordance of positive scalar curvature metrics via index invariants on submanifolds.

From a technical standpoint, the central novelty of this thesis is that we use Yu's localization algebras in combination with the description of K-theory for graded $\mathrm{C}^{*}$ algebras due to Trout. This formalism allows direct definitions of all the invariants we consider in terms of the functional calculus of the Dirac operator and enables us to give concise proofs of the product formulas. It also allows us to consistently work in the setting of real K-theory.
\end{abstract}





\section{Acknowledgments}

My primary thanks go to my thesis advisor Thomas Schick for suggesting fascinating topics, sharing his knowledge during many fruitful discussions, and for his continuous encouragement as well as allowing me great freedom in my work.

I also would like to thank my second advisor Ralf Meyer for the discussions during the annual meetings and for the useful comments on the preprint of my article that forms the base of this thesis.

Furthermore, I thank my family and friends who have accompanied and supported me in my personal and professional development. I am indebted to Elisabeth for being ever so kind and understanding, my mother and grandparents for their unconditional support, and my father who always fostered my intellectual curiosity.

Funding acknowledgment: This thesis was supported by the German Research Foundation (DFG) through the Research Training Group 1493 "Mathematical structures in modern quantum physics" at the University of Göttingen. 



\section{Contents}

$\begin{array}{lr}\text { Introduction } & 1\end{array}$

1 Preliminaries $\quad 11$

1.1 K-theory of $\mathrm{C}^{*}$-algebras . . . . . . . . . . . . . . . . . . . . . . . . 11

1.1.1 Graded $\mathrm{C}^{*}$-algebras . . . . . . . . . . . . . . . . 11

1.1 .2 External Product . . . . . . . . . . . . . . . . . . 12

1.1 .3 Bott periodicity . . . . . . . . . . . . . 15

1.1.4 Long exact sequences . . . . . . . . . . . . . . . . . . . . . . . . . . . . . 16

1.2 Spin geometry. . . . . . . . . . . . . . . . . . . . . . . . . . . . . . . . . . .

1.2 .1 Spin structures . . . . . . . . . . . . . . . 17

1.2.2 The spinor Dirac operator . . . . . . . . . . . . . . 17

1.3 Metric considerations on non-compact manifolds . . . . . . . . . . 18

1.3.1 Admissible metrics on manifolds with boundary . . . . . . . . . 18

1.3.2 Concordance of positive scalar curvature metrics . . . . . . . . . 20

1.3.3 Attaching a cylindrical end . . . . . . . . . . . . . 21

1.4 Yu's localization algebras and K-homology . . . . . . . . . . . . . 21

1.4.1 Localization algebras . . . . . . . . . . . . . . . 21

1.4.2 Functoriality of localization algebras . . . . . . . . . . 25

1.4.3 Analytic K-homology . . . . . . . . . . . . 28

2 Secondary large-scale index theory for psc 31

2.1 Partial structure groups . . . . . . . . . . . . . . . . . . . . . . . . . 31

2.1.1 Generalized Higson-Roe sequences . . . . . . . . . . . . . . . 31

2.1.2 Mayer-Vietoris sequences . . . . . . . . . . . . . . . 32

2.1.3 Flasque spaces and suspension isomorphisms . . . . . . . . . 37

2.1.4 External products .................... 38

2.2 Definition of secondary invariants . . . . . . . . . . . . . . . . . . . . . . . . . . . . . . . 49

2.2.1 The fundamental class via localization algebras . . . . . . . . . . 40

2.2.2 The partial $\rho$-invariant and the localized coarse index . . . . . . 41

2.2.3 The coarse APS-index for partial psc at the boundary . . . . . . 43

2.2.4 The index difference . . . . . . . . . . . . . . . . . 44

2.3 Product formulas for secondary invariants . . . . . . . . . . . . . . . . . . . . . 45

2.3.1 "Boundary of Dirac is Dirac" . . . . . . . . . . . . . . . . . . 47

2.3.2 Hypereuclidean manifolds ... . . . . . . . . . . 50

2.4 Secondary index theorems . . . . . . . . . . . . . . 51

2.4.1 Partitioned manifold index theorems . . . . . . . . . . . . 51

2.4 .2 The coarse APS-index theorem . . . . . . . . . . . . . 58

2.5 Explicit descriptions in terms of projections and unitaries . . . . . . 61

2.5.1 K-theory of trivially graded $\mathrm{C}^{*}$-algebras . . . . . . . . . . . 61 
2.5 .2 Reduced spinor bundles . . . . . . . . . . . . . . . . . . 62

2.5.3 Local index classes in terms of projections and unitaries . . . . 63

3 Secondary invariants on compact manifolds $\quad 67$

3.1 Mapping positive scalar curvature to analysis . . . . . . . . . . . 67

3.2 Stability of higher secondary invariants . . . . . . . . . . . . . . 70

3.3 From closed manifolds to non-compact complete manifolds . . . . . . . . 71

4 Obstructions via submanifolds

4.1 Secondary obstructions via submanifolds of low codimension . . . . . . 75

4.1 .1 Codimension one . . . . . . . . . . . . . . . 75

4.1 .2 Codimension two . . . . . . . . . . . . . . . . 76

4.2 A multi-partitioned manifold index theorem . . . . . . . . . . . . . . 78

4.2.1 Multi-partitioned manifolds . . . . . . . . . . . . . . . . 81

4.2.2 Fiber bundles over aspherical manifolds . . . . . . . . . . . . 82

4.3 The failure of secondary multi-partitioned manifold index theorems . . . 83

\section{Bibliography}




\section{Introduction}

A major theme in differential geometry and geometric topology is the study of Riemannian metrics of positive scalar curvature $(p s c)$. The fundamental questions in this area are both existence, that is, whether a given smooth manifold admits a metric of psc, and classification, that is, if it does, how many different ones there are with respect to a suitable equivalence relation.

The most successful approach to these problems has been through index theory of the spinor Dirac operators on spin manifolds. The main ingredient is the SchrödingerLichnerowicz formula,

$$
\not{D}^{2}=\nabla^{*} \nabla+\frac{\text { scal }}{4},
$$

which shows that in the presence of uniformly psc, the spinor Dirac operator $\not{D}$ of a spin manifold is invertible. In particular, a closed spin manifold where the Fredholm index of $\mathscr{D}$ is non-zero does not support a metric of psc. This fact was first established by Lichnerowicz [Lic63], who used it together with the Atiyah-Singer index theorem to provide topological obstructions to the existence of psc metrics on highdimensional closed manifolds. Later, this method was expanded in different directions via refined versions of the index, notably by Hitchin [Hit74], Gromov and Lawson [GL83], Rosenberg [Ros83], and Roe [Roe96]. These refined indices lie in different K-theory groups. All have the common feature that they vanish in the presence of psc and hence provide information on the existence problem. Hitchin [Hit74] also constructed an index difference associated to two metrics of psc, a secondary index whose non-vanishing is an obstruction to concordance of positive scalar curvature metrics.

In this thesis, we approach the whole subject from the point of view of Roe's coarse index theory, which was designed to deal with non-compact manifolds. The coarse index of the Dirac operator on a complete spin manifold $X$, possibly endowed with a suitable action of a discrete group $\Gamma$, resides in the $\mathrm{K}$-theory group $\mathrm{K}_{*}\left(\mathrm{C}_{\Gamma}^{*}(X)\right)$, where $\mathrm{C}_{\Gamma}^{*}(X)$ is the equivariant Roe algebra of $X$. The latter is a certain $\mathrm{C}^{*}$-algebra that encodes information both about the group action and the large-scale geometry of $X$. There is a long exact sequence of abelian groups,

$$
\cdots \rightarrow \mathrm{K}_{*+1}\left(\mathrm{C}_{\Gamma}^{*}(X)\right) \stackrel{\partial^{\Gamma}}{\longrightarrow} \mathrm{S}_{*}^{\Gamma}(X) \rightarrow \mathrm{K}_{*}^{\Gamma}(X) \stackrel{\operatorname{Ind}^{\Gamma}}{\longrightarrow} \mathrm{K}_{*}\left(\mathrm{C}_{\Gamma}^{*}(X)\right) \rightarrow \cdots,
$$

where $\mathrm{K}_{*}^{\Gamma}(X)$ is equivariant analytic K-homology and $\mathrm{S}_{*}^{\Gamma}(X)$ is the analytic structure group of Higson and Roe. From this abstract point of view, the coarse index is obtained by applying the index map $\operatorname{Ind}^{\Gamma}: \mathrm{K}_{n}^{\Gamma}(X) \rightarrow \mathrm{K}_{n}\left(\mathrm{C}_{\Gamma}^{*}(X)\right)$ to the K-homological fundamental class $[X] \in \mathrm{K}_{n}^{\Gamma}(X)$. If the $\Gamma$-invariant Riemannian metric $g$ on $X$ has uniformly positive scalar curvature (upsc), then there is a secondary invariant, the $\rho$-invariant $\rho^{\Gamma}(g) \in \mathrm{S}_{n}^{\Gamma}(X)$ which depends on the metric $g$ and lifts the fundamental class to the structure group. By exactness, this shows that the index is zero, but 
more so, a quantitative reason for its vanishing. Two psc metrics with different $\rho$ invariants cannot be bordant in a suitable sense. For two uniform psc metrics $g_{0}$, $g_{1}$, there is a secondary index inspired by Hitchin's index difference, the coarse index difference $\operatorname{Ind}_{\text {diff }}^{\Gamma}\left(g_{0}, g_{1}\right) \in \mathrm{K}_{n+1}\left(\mathrm{C}_{\Gamma}^{*}(X)\right)$. This is related to the $\rho$-class via the formula $\partial^{\Gamma}\left(\operatorname{Ind}_{\text {diff }}^{\Gamma}\left(g_{0}, g_{1}\right)\right)=\rho^{\Gamma}\left(g_{0}\right)-\rho^{\Gamma}\left(g_{1}\right)$. These secondary invariants have been the focus of intensive study in the recent past, see for instance [HR10; Sie12a; XY14a; PS14; WY13; XY14b; XY13].

In the framework of coarse index theory, the index difference of two upsc metrics $g_{0}, g_{1}$ can be defined as a "localized" index associated to a metric on $X \times \mathbb{R}$ which interpolates between $g_{0}$ and $g_{1}$ (but does not necessarily have upsc along the way), see Subsection 2.2.4. This has led us to consider complete Riemannian metrics which have upsc outside a given $\Gamma$-invariant subset $Z \subseteq X$ and develop a secondary index theory adopted to such situations. In fact, Roe has already shown that if the metric has upsc outside $Z$, then the coarse index can be "localized" to the subset $Z$ by constructing an index class in $\mathrm{K}_{*}\left(\mathrm{C}_{\Gamma}^{*}(Z \subset X)\right)$, see [Roe96; Roe16]. Here $\mathrm{C}_{\Gamma}^{*}(Z \subset X)$ denotes the ideal in the Roe algebra generated by operators supported near $Z$. Based on this observation, we introduce and study partial secondary invariants associated to metrics which have upsc outside a given subset $Z \subseteq X$. This includes the construction of a partial structure group $\mathrm{S}_{*}^{\Gamma}(X / / Z)$ together with partial $\rho$-classes $\rho_{Z}^{\Gamma}(g) \in \mathrm{S}_{n}^{\Gamma}(X / / Z)$ for Riemannian metrics $g$ which have upsc outside the subset $Z$. The partial structure group interpolates between the Higson-Roe structure group and K-homology: There are identifications $\mathrm{S}_{*}^{\Gamma}(X / / \emptyset)=\mathrm{S}_{*}^{\Gamma}(X)$ and $\mathrm{S}_{*}^{\Gamma}(X / / X)=\mathrm{K}_{*}^{\Gamma}(X)$ such that $\rho_{\emptyset}^{\Gamma}(g)=\rho^{\Gamma}(g)$ and $\rho_{X}^{\Gamma}(g)=[X]^{\Gamma}$.

The partial structure group fits into a long exact sequence

$$
\mathrm{K}_{*+1}\left(\frac{\mathrm{C}_{\Gamma}^{*}(X)}{\mathrm{C}_{\Gamma}^{*}(Z \subset X)}\right) \stackrel{\partial_{/ / Z}^{\Gamma}}{\longrightarrow} \mathrm{S}_{*}^{\Gamma}(X / / Z) \stackrel{q_{Z, X}^{\Gamma}}{\longrightarrow} \mathrm{K}_{*}^{\Gamma}(X) \stackrel{\operatorname{Ind}_{/ / Z}^{\Gamma}}{\longrightarrow} \mathrm{K}_{*+1}\left(\frac{\mathrm{C}_{\Gamma}^{*}(X)}{\mathrm{C}_{\Gamma}^{*}(Z \subset X)}\right) .
$$

If a metric $g$ has upsc outside $Z$, so that $\rho_{Z}^{\Gamma}(g) \in \mathrm{S}_{n}^{\Gamma}(X / / Z)$ exists, then we have $q_{Z, X}^{\Gamma}\left(\rho_{Z}^{\Gamma}(g)\right)=[X]^{\Gamma}$. In particular, the index class $\operatorname{Ind}_{/ / Z}^{\Gamma}\left([X]^{\Gamma}\right)$ is an obstruction to the existence of a metric of upsc outside $Z$. Moreover, given two metrics $g_{0}, g_{1}$ of upsc outside $Z$, we introduce a partial index difference,

$$
\operatorname{Ind}_{\text {diff }}^{\Gamma}\left(g_{0}, g_{1} / / Z\right) \in \mathrm{K}_{n+1}\left(\frac{\mathrm{C}_{\Gamma}^{*}(X)}{\mathrm{C}_{\Gamma}^{*}(Z \subset X)}\right),
$$

which satisfies

$$
\partial_{/ / Z}^{\Gamma}\left(\operatorname{Ind}_{\mathrm{diff}}^{\Gamma}\left(g_{0}, g_{1} / / Z\right)\right)=\rho_{Z}^{\Gamma}\left(g_{0}\right)-\rho_{Z}^{\Gamma}\left(g_{1}\right) .
$$

We will now explain in which sense the partial $\rho$-class and the index difference can distinguish metrics of psc. Since the receptacles of the coarse indices as well as the (partial) $\rho$-classes are sensitive to the coarse type of the manifold, coarse index theory cannot be used to compare two arbitrary complete Riemannian metrics on a non-compact manifold. We need to restrict the large-scale structure of the metrics we allow in our considerations. Our approach to this is to fix a proper reference metric on $X$ and consider only those Riemannian metrics that uniformly dominate the chosen 
reference metric (see Section 1.3 for details). Given an implicitly fixed reference metric, we then denote by $\mathcal{R}(X)$ the set of all those Riemannian metrics on $X$. The subset of $\mathcal{R}(X)$ of those metrics with upsc outside $Z$ is denoted by $\mathcal{R}_{Z}^{+}(X)$. If $X$ is endowed with a $\Gamma$-action, we denote the subset of invariant metrics by $\mathcal{R}_{Z}^{+}(X)^{\Gamma}$.

Definition 1.3.5. Let $g_{0}, g_{1} \in \mathcal{R}_{Z}^{+}(X)^{\Gamma}$. We call $g_{0}$ and $g_{1}$ concordant relative to $Z$ if there exists a Riemannian metric $h \in \mathcal{R}_{Z \times \mathbb{R}}^{+}(X \times \mathbb{R})^{\Gamma}$ such that

(i) $h \uparrow X \times(-\infty, 0]=g_{0} \oplus \mathrm{d} t^{2}$

(ii) $h \uparrow X \times[1, \infty)=g_{1} \oplus \mathrm{d} t^{2}$.

If $Z=\emptyset, g_{0}$ and $g_{1}$ are called concordant.

For closed manifolds and $Z=\emptyset$, this reduces to the usual notion of concordance for metrics of psc.

The partial index difference $\operatorname{Ind}_{\text {diff }}^{\Gamma}\left(g_{0}, g_{1}\right)$ vanishes if $g_{0}$ and $g_{1}$ are concordant relative to $Z$ in the sense of the following definition. So, the partial $\rho$-class is an invariant of relative concordance.

One of our initial motivations for the partial $\rho$-classes was to use them as a formal tool to deal with the index difference and Atiyah-Patodi-Singer-type indices. However, they are also of intrinsic interest in the study of certain positive scalar curvature phenomena on non-compact manifolds. Indeed, as will be explained below, using these invariants we are able to construct examples of Riemannian metrics of upsc on non-compact manifolds which are not concordant relative to certain subsets.

\section{Outline of main results}

In Chapter 2, the main part of this thesis, we develop the theory of secondary invariants for Riemannian metrics of partial positive scalar curvature. We prove all results for complete spin manifolds endowed with a proper and free (but not necessarily cocompact) action of a discrete group. Moreover, all our constructions consistently work in all dimensions and irrespective of whether we choose to work with real or complex Ktheory. This is in contrast to much of the previous literature on the subject such as [PS14; XY14b], where only the complex case is treated by considering even- and odd-dimensional manifolds separately.

\section{Product formulas}

The central principle of our theory is the following external product formula for partial $\rho$-classes.

Theorem 2.3.1. For $i \in\{1,2\}$, let $X_{i}$ be a complete spin manifold endowed with a free and proper action of a discrete group $\Gamma_{i}$. Let $Z_{1} \subseteq X_{1}$ be some $\Gamma_{1}$-invariant subset. Set $X:=X_{1} \times X_{2}$ and $Z:=Z_{1} \times X_{2}$ and $\Gamma:=\Gamma_{1} \times \Gamma_{2}$. 
Then for all $g_{1} \in \mathcal{R}_{Z_{1}}^{+}\left(X_{1}\right)^{\Gamma_{1}}$ and $g_{2} \in \mathcal{R}\left(X_{2}\right)^{\Gamma_{2}}$ with $g:=g_{1} \oplus g_{2} \in \mathcal{R}_{Z}^{+}(X)$, the following product formula holds:

$$
\rho_{Z}^{\Gamma}(g)=\rho_{Z_{1}}^{\Gamma_{1}}\left(g_{1}\right) \otimes\left[X_{2}\right]^{\Gamma_{2}} .
$$

The external product $\mathrm{S}_{*}^{\Gamma_{1}}\left(X_{1} / / Z_{1}\right) \otimes \mathrm{K}_{*}^{\Gamma_{2}}\left(X_{2}\right) \rightarrow \mathrm{S}_{*}^{\Gamma}(X / / Z)$ we use here is defined in Subsection 2.1.4.

For $Z=X$, this theorem reduces to the classical product formula for fundamental classes in K-homology. For $Z=\emptyset$, it yields a new proof of the product formula for the $\rho$-class,

$$
\rho^{\Gamma_{1}}\left(g_{1}\right) \otimes\left[X_{2}\right]^{\Gamma_{2}}=\rho^{\Gamma_{1} \times \Gamma_{2}}\left(g_{1} \oplus g_{2}\right) .
$$

The formula (5) was proved in the thesis of Siegel [Sie12a], where a construction of the structure group in terms of a customized notion of Kasparov cycles is used. However, in Siegel's construction the compatibility between the exterior product and the MayerVietoris boundary map appears to be not straightforward. Siegel's approach has also been studied by Zenobi [Zen14] with a focus on the signature operator and secondary invariants associated to homotopy equivalences. Moreover, the product formula (5) can be deduced using the geometric picture of the structure group due to DeeleyGoffeng [DG15]. Another discussion of (5) is implicit in the work of Xie-Yu [XY14b, pp. 838-839] using Yu's localization algebras.

Our approach is a variant of Xie-Yu's, entailing technical simplifications in the construction of the invariants and in the proof of the product formula. Indeed, similarly as in [XY14b], we use variants of Yu's localization algebra to construct the partial structure groups and realize the Higson-Roe exact sequence (1) as well as the generalized version (2). The main novelty is that we combine Yu's localization algebra with the description of $\mathrm{K}$-theory for graded $\mathrm{C}^{*}$-algebras due to Trout [Tro00]. This formalism allows tautological definitions of the fundamental classes and of the (partial) $\rho$-classes in terms of the functional calculus of the Dirac operator, see Section 2.2. In order to treat all dimensions at once, we consider a $\mathrm{Cl}_{n}$-linear variant of the localization algebras (see Section 1.4) and consistently work with the $\mathrm{Cl}_{n}$-linear Dirac operator. In particular, our approach also works straightforwardly in the setting of real K-theory. Using this setup we give a concise and self-contained proof of the product formulas, see Section 2.3.

\section{Secondary partitioned manifold index theorems}

It is crucial for applications that the external product is compatible with Mayer-Vietoris boundary maps. In our construction, this is the case because the external product we use is induced by the external product in K-theory, where compatibility with boundary maps can be checked abstractly. Together with the product formula we obtain a partitioned manifold index theorem for the partial $\rho$-class as follows:

Let $W$ be a complete spin manifold. Let $X \subset W$ be a closed submanifold of codimension one with trivial normal bundle. We suppose that $W \backslash X$ has two connected components and denote the closures of the connected components of $W \backslash X$ by $W_{ \pm}$. If $W$ is endowed with a $\Gamma$-action, we additionally require that $X, W_{-}$and $W_{+}$are all 
$\Gamma$-invariant subsets. In this situation, we say that $W$ is partitioned by $X$. Moreover, a Riemannian metric $h \in \mathcal{R}(W)$ is partitioned by $g \in \mathcal{R}(X)$ if near $X$ the metric $h$ can be written as $g \oplus \mathrm{d} t^{2}$ with respect to a tubular neighborhood of $X$.

Theorem 2.4.6. Let $W$ be a complete spin manifold endowed with a free and proper $\Gamma$ action and suppose that it is partitioned by $X$. Let $Z \subseteq W$ be a closed $\Gamma$-invariant subset that is admissible with respect to $W_{+}$and suppose that $h \in \mathcal{R}_{Z}^{+}(W)^{\Gamma}$ is partitioned by $g \in \mathcal{R}_{Z \cap X}^{+}(X)^{\Gamma}$. Then the Mayer-Vietoris boundary map

$$
\partial_{\mathrm{MV}}: \mathrm{S}_{*+1}^{\Gamma}(W / / Z) \rightarrow \mathrm{S}_{*}^{\Gamma}(X / / Z \cap X)
$$

associated to the cover $W=W_{+} \cup W_{-}$satisfies

$$
\partial_{\mathrm{MV}}\left(\rho_{Z}^{\Gamma}(h)\right)=\rho_{Z \cap X}^{\Gamma}(g) .
$$

Here the subset $Z$ is called "admissible" if it satisfies a suitable large-scale condition, see Definition 2.4.2. Valid choices include $Z=X, Z=\emptyset$ and $Z=W_{-} \backslash(X \times(-\varepsilon, 0])$ (with respect to a tubular neighborhood).

In the case $Z=\emptyset$, we obtain a new proof of the secondary partitioned manifold index theorem of Piazza-Schick [PS14, Theorem 1.22] for all dimensions. We also have a partitioned manifold index theorem for the index difference (see Corollary 2.4.9).

\section{Coarse APS-index theory}

The other main index theorem of [PS14], the "delocalized Atiyah-Patodi-Singer (APS) index theorem", is a consequence of our partitioned manifold index theorem for partial secondary invariants. Before explaining this, we first state the theorem and some generalizations. Consider a complete spin manifold $Y$ with boundary $\partial Y=X$ endowed with a free, proper $\Gamma$-action. Suppose that the inclusion $X \hookrightarrow Y$ is a coarse equivalence. Then for every $g \in \mathcal{R}^{+}(X)^{\Gamma}$, there is an APS-type index $\operatorname{Ind}_{\text {APS }}^{\Gamma}(Y, X, g) \in \mathrm{K}_{n+1}\left(\mathrm{C}_{\Gamma}^{*}(X)\right)$ whose non-vanishing is an obstruction to extending $g$ to a metric of upsc on $Y$. This index has already been studied in [PS14; XY14b]. In addition, we construct in Definition 2.4 .13 a " $\rho$-invariant of the null-bordism $Y$ " as an element $\rho^{\Gamma}(Y) \in \mathrm{S}_{*}^{\Gamma}(X)$ which maps to the fundamental class $[X]^{\Gamma} \in \mathrm{K}_{n}^{\Gamma}(X)$. In particular, the index $\operatorname{Ind}^{\Gamma}(X)$ vanishes; this is a variant of bordism invariance for the coarse index, compare [Wul12]. These invariants are related in the following way:

Corollary 2.4.16. For every $g \in \mathcal{R}^{+}(X)^{\Gamma}$ the following identity holds:

$$
\partial^{\Gamma}\left(\operatorname{Ind}_{\mathrm{APS}}^{\Gamma}(Y, X, g)\right)=\rho^{\Gamma}(g)-\rho^{\Gamma}(Y) \in \mathrm{S}_{n}^{\Gamma}(X) .
$$

This is a refinement of the original delocalized APS-index theorem ([PS14, Theorem 1.14]) because we have an equality in $\mathrm{S}_{*}^{\Gamma}(X)$ instead of merely in $\mathrm{S}_{*}^{\Gamma}(Y)$. In fact, the element $\rho^{\Gamma}(Y)$ vanishes after pushing it forward to $\mathrm{S}_{*}^{\Gamma}(Y)$, and thus the original result is recovered. As a further generalization, we also construct an APS-index $\operatorname{Ind}_{\mathrm{APS}}^{\Gamma}(Y, X / / Z, g) \in \mathrm{K}_{n+1}\left(\mathrm{C}_{\Gamma}^{*}(X) / \mathrm{C}_{\Gamma}^{*}(Z \subset X)\right)$ for Riemannian metrics of upsc outside a subset $Z$ on the boundary, that is, $g \in \mathcal{R}_{Z}^{+}(X)^{\Gamma}$, see Subsection 2.2.3. We then establish the following coarse APS-index theorem for partial upsc: 
Theorem 2.4.15. For every $g \in \mathcal{R}_{Z}^{+}(X)^{\Gamma}$ the following identity holds:

$$
\partial_{/ / Z}^{\Gamma}\left(\operatorname{Ind}_{\mathrm{APS}}^{\Gamma}(Y, X / / Z, g)\right)=\rho_{Z}^{\Gamma}(g)-q_{0, Z}^{\Gamma}\left(\rho^{\Gamma}(Y)\right) \in \mathrm{S}_{n}^{\Gamma}(X / / Z) .
$$

Here $q_{0, Z}^{\Gamma}$ is a natural map $\mathrm{S}_{*}^{\Gamma}(X) \rightarrow \mathrm{S}_{*}^{\Gamma}(X / / Z)$, and the boundary map is the one appearing in the generalized Higson-Roe sequence (2). The proof of Theorem 2.4.15 (and hence Corollary 2.4.16) applies the partitioned manifold index theorem (Theorem 2.4.6) to the partitioned manifold $Y_{\infty}:=Y \cup_{X} X \times \mathbb{R}_{\geqslant 0}$ obtained by gluing an infinite cylindrical end to $Y$ along its boundary.

The index difference (3) can be realized as a special case of the coarse APS-index (see Subsection 2.2.4). Then formula (4) follows from Theorem 2.4.15 (see Corollary 2.4.19). In addition, Theorem 2.4.15 implies that the partial $\rho$-class is a coarse bordism invariant in a suitable sense (see Corollary 2.4.20).

\section{Applications}

As a sample application, we recall the coarse-geometric construction of higher secondary invariants associated to metrics of psc on compact spin manifolds. This is done by passing to the universal covering and considering equivariant $\rho$-classes, APS-indices and index differences with respect to the deck-transformation action of the fundamental group. More precisely, let $M$ be a closed spin manifold together with a continuous map $u: M \rightarrow \mathrm{B} \Gamma$. Let $\tilde{M} \rightarrow M$ be the $\Gamma$-covering classified by $u$. Then for every Riemannian metric $g$ on $M$ of psc, we define the higher $\rho$-invariant $\rho^{u}(g) \in \mathrm{S}(\Gamma)$ as the push-forward of $\rho^{\Gamma}(\tilde{g}) \in \mathrm{S}_{n}^{\Gamma}(\tilde{M})$ to the universal structure group for $\Gamma$ via $\tilde{u}: \tilde{M} \rightarrow \mathrm{E} \Gamma$. Since $\tilde{M}$ is $\Gamma$-cocompact, there is a canonical isomorphism $\mathrm{K}_{*}\left(\mathrm{C}_{\Gamma}^{*}(\tilde{M})\right)=\mathrm{K}_{*}\left(\mathrm{C}_{\mathrm{r}}^{*} \Gamma\right)$, where $\mathrm{C}_{\mathrm{r}}^{*} \Gamma$ denotes the reduced group $\mathrm{C}^{*}$-algebra. Hence we may consider $\alpha^{u}(M):=$ $\operatorname{Ind}^{\Gamma}(\tilde{M}) \in \mathrm{K}_{n}\left(\mathrm{C}_{\mathrm{r}}^{*} \Gamma\right)$ and $\alpha_{\mathrm{diff}}^{u}\left(g_{0}, g_{1}\right):=\operatorname{Ind}_{\text {diff }}^{\Gamma}\left(\tilde{g}_{0}, \tilde{g}_{1}\right) \in \mathrm{K}_{n+1}\left(\mathrm{C}_{\mathrm{r}}^{*} \Gamma\right)$, the latter for $g_{0}, g_{1} \in \mathcal{R}^{+}(M)$. If $u$ is the classifying map of the universal covering, we just write $\rho^{\Gamma}(g), \alpha^{\Gamma}(M)$ and $\alpha_{\text {diff }}^{\Gamma}\left(g_{0}, g_{1}\right)$.

The most conceptual approach to these invariants is via a transformation from Stolz' positive scalar curvature sequence to the universal Higson-Roe sequence associated to some discrete group $\Gamma$, as it has been established by Piazza-Schick and Xie-Yu:

Theorem 3.1.13 ([PS14; XY14b]). We have a well-defined commutative diagram:

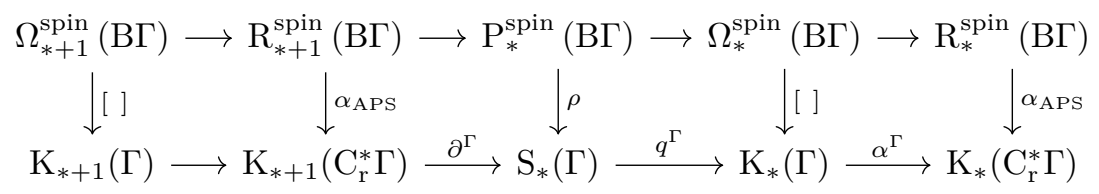

In Section 3.1, we reprove this result based on the secondary coarse index theory we have established in Chapter 2. In particular, this includes a proof of bordism invariance of the higher APS-index based solely on methods from coarse index theory. Since all our methods are designed to work with real K-theory, our approach to Theorem 3.1.13 
also establishes the transformation from the Stolz sequence to the real Higson-Roe sequence.

The main application of secondary invariants for positive scalar curvature is to distinguish metrics of upsc up to concordance or bordism. Of course, this is only interesting if there indeed exist pairs of metrics of upsc which are distinguished by the $\rho$-class or the index difference. If $M$ is a closed spin manifold that admits a metric of psc and $\Gamma:=\pi_{1}(M)$ has torsion and satisfies the analytic Novikov conjecture, then there always exist metrics $g_{0}, g_{1} \in \mathcal{R}^{+}(M)$ with $\rho^{\Gamma}\left(g_{0}\right) \neq \rho^{\Gamma}\left(g_{1}\right) \in \mathrm{S}_{n}(\Gamma)$. This statement follows, for example, from work of Weinberger-Yu [WY13], where a more quantative version based on the amount of torsion in $\Gamma$ is established. Since such examples exist for closed manifolds, our structural results like the product formulas and the partitioned manifold index theorems can be utilized to construct further examples of upsc metrics that can be distinguished by our secondary index invariants. In the following, we present several incarnations of this idea.

The first result is a corollary of the secondary partitioned manifold index theorem, Theorem 2.4.6.

Corollary 3.3.1. Let $M$ be a closed spin manifold together with a map $u: M \rightarrow \mathrm{B} \Gamma$ and $g_{0}, g_{1} \in \mathcal{R}^{+}(M)$ such that $\rho^{u}\left(g_{0}\right) \neq \rho^{u}\left(g_{1}\right)$. Let $W$ be a complete spin manifold with $h_{0}, h_{1} \in \mathcal{R}^{+}(W)$ such that $W$ is partitioned by $M$ and $h_{i}$ is partitioned by $g_{i}$, $i=0,1$ (see Definition 2.4.1). Suppose that $u$ extends to a map $W \rightarrow \mathrm{B} \Gamma$. Then $h_{0}$ and $h_{1}$ are not concordant relative to $W_{-}\left(\right.$or $\left.W_{+}\right)$, where $W_{ \pm}$are the connected components of $W \backslash M$.

Before stating a further corollary, we recall a geometric notion due to Gromov [Gro93]. A complete Riemannian manifold $Y$ is called hypereuclidean if it admits a proper Lipschitz map $Y \rightarrow \mathbb{R}^{q}$ of degree 1 into some Euclidean space $\mathbb{R}^{q}$ (if this is the case, then $q=\operatorname{dim} Y$ ). We say that $Y$ is stably hypereuclidean if $Y \times \mathbb{R}^{k}$ is hypereuclidean for some $k \geqslant 0$.

Corollary 3.3.6. Let $M$ be a closed spin manifold together with a map $u: M \rightarrow \mathrm{B} \Gamma$ and $g_{0}, g_{1} \in \mathcal{R}^{+}(M)$. Moreover, let $Y$ be a complete spin manifold, $g_{Y} \in \mathcal{R}(Y)$ and $Z \subseteq Y$ some subset. Suppose that

(i) $\alpha_{\text {diff }}^{u}\left(g_{0}, g_{1}\right) \neq 0 \in \mathrm{K}_{n+1}\left(\mathrm{C}_{\mathrm{r}}^{*} \Gamma\right)$,

(ii) $g_{i} \oplus g_{Y} \in \mathcal{R}^{+}(M \times Y)$ for $i=0,1$,

(iii) $\left(Y, g_{Y}\right)$ is stably hypereuclidean,

(iv) $Z$ is coarsely negligible in $Y$.

Then the metrics $g_{0} \oplus g_{Y}$ and $g_{1} \oplus g_{Y}$ are not concordant on $M \times Y$ relative to $M \times Z$.

Here we say that a subset $Z \subseteq Y$ is coarsely negligible if the inclusion map coarsely factors through a flasque space (see Definition 3.3.2). Examples of coarsely negligible subsets include compact subsets of complete Riemannian manifolds and half-spaces in Euclidean spaces. 
By a result of Dranishnikov [Dra06, Theorem 3.5], the universal covering of an aspherical manifold is stably hypereuclidean if the fundamental group has finite asymptotic dimension. We use this to conclude the following product result for the higher $\rho$-invariant for closed manifolds.

Corollary 3.2.2. Let $M_{i}$ be closed spin, $u_{i}: M_{i} \rightarrow \mathrm{B} \Gamma$ and $g_{i} \in \mathcal{R}^{+}\left(M_{i}\right), i=0,1$, such that $\rho^{u_{0}}\left(g_{0}\right) \neq \rho^{u_{1}}\left(g_{1}\right)$. Let $N$ be a closed aspherical spin q-manifold such that $\Lambda=\pi_{1}(N)$ has finite asymptotic dimension. Let $g_{N} \in \mathcal{R}(N)$ such that $g_{i} \oplus g_{N} \in$ $\mathcal{R}^{+}(M \times N)$ for $i=0,1$. Then $\rho^{u_{0} \times \operatorname{id}_{N}}\left(g_{0} \oplus g_{N}\right) \neq \rho^{u_{1} \times \operatorname{id}_{N}}\left(g_{1} \oplus g_{N}\right)$.

Going in a slightly different direction, we apply our methods to obtain obstructions to concordance on closed manifolds via secondary index invariants on submanifolds. To understand the context of this, note that Hanke-Pape-Schick [HPS15] have shown that, under suitable conditions, the $\alpha$-invariant of a codimension two submanifold is an obstruction to positive scalar curvature on an ambient closed spin manifold. We establish a variety of modifications of this result. For instance, we have a secondary codimension two obstruction as follows.

Theorem 4.1.3. Let $M$ be a closed spin manifold and $N \subseteq M$ a closed submanifold of codimension two with trivial normal bundle. Fix a tubular neighborhood $t: N \times \mathrm{D}_{\varepsilon} \hookrightarrow M$. Suppose that the inclusion induces an injection $\Lambda:=\pi_{1} N \hookrightarrow \pi_{1} M=: \Gamma$ and a surjection $\pi_{2} N \rightarrow \pi_{2} M$. Let $g_{0}, g_{1} \in \mathcal{R}^{+}(M)$ such that $t^{*}\left(g_{i}\right)=g_{N, i} \oplus g_{\mathrm{D}, i}$, where $g_{\mathrm{D}, i}$ is cylindrical near the boundary for $i \in\{0,1\}$. If $\rho^{\Lambda}\left(g_{N, 0}\right) \neq \rho^{\Lambda}\left(g_{N, 1}\right)$ in $\mathrm{S}_{n-2}(\Lambda)$, then $g_{0}$ and $g_{1}$ are not concordant on $M$.

The proof of this statement relies on the secondary partitioned manifold index theorem and the language of partial $\rho$-classes. Moreover, we attempt to generalize both primary and secondary obstructions to codimensions other than two. The picture is most complete for codimension one:

Theorem 4.1.1. Let $M$ be a closed spin manifold and $\Gamma=\pi_{1} M$. Let $N \subset M$ be a closed submanifold of codimension 1 with trivializable normal bundle. Suppose that the inclusion induces an injection $\pi_{1} N=: \Lambda \hookrightarrow \Gamma$. Fix a tubular neighborhood $\iota: N \times(-\varepsilon, \varepsilon) \hookrightarrow M$. There exists a commutative diagram,

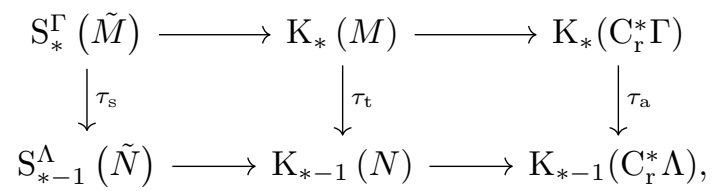

with the following properties:

(i) $\tau_{\mathrm{t}}([M])=[N]$,

(ii) $\tau_{\mathrm{a}}\left(\alpha^{\Gamma}(M)\right)=\alpha^{\Lambda}(N)$,

(iii) $\tau_{\mathrm{s}}\left(\rho^{\Gamma}(\tilde{g})\right)=\rho^{\Lambda}\left(\tilde{g}_{N}\right)$ for all $g_{M} \in \mathcal{R}^{+}(M)$ with producture structure $\iota^{*} g=g_{N} \oplus \mathrm{d} t^{2}$ on the tubular neighborhood of $N$, 
(iv) $\tau_{\mathrm{a}}\left(\alpha_{\text {diff }}^{\Gamma}\left(g_{0}, g_{1}\right)\right)=\alpha_{\text {diff }}^{\Lambda}\left(g_{N, 0}, g_{N, 1}\right)$ for all $g_{0}, g_{1} \in \mathcal{R}^{+}(M)$ with product structure $\iota^{*} g_{i}=g_{N, i} \oplus \mathrm{d} t^{2}, i=0,1$, on the tubular neighborhood of $N$.

Corollary 4.1.2. Suppose that the hypotheses of Theorem 4.1.1 hold. Then:

- If $\alpha^{\Lambda}(N) \neq 0$ in $\mathrm{K}_{*}\left(\mathrm{C}_{\mathrm{r}}^{*} \Lambda\right)$, then $\alpha^{\Gamma}(M) \neq 0$ and $M$ does not admit a metric of positive scalar curvature.

For $i \in\{0,1\}$ given $g_{i} \in \mathcal{R}^{+}(M)$ with product structure $g_{i}=g_{N, i} \oplus \mathrm{d} t^{2}, i=0,1$, near $N$, we deduce:

- If $\alpha_{\mathrm{diff}}^{\Lambda}\left(g_{N, 0}, g_{N, 1}\right) \neq 0$, then $\alpha_{\mathrm{diff}}^{\Gamma}\left(g_{M, 0}, g_{M, 1}\right) \neq 0$ and $g_{0}$ is not concordant to $g_{1}$ on $M$.

- If $\rho^{\Lambda}\left(g_{N, 0}\right) \neq \rho^{\Lambda}\left(g_{N, 1}\right)$, then $\rho^{\Gamma}\left(g_{0}\right) \neq \rho^{\Gamma}\left(g_{1}\right)$.

Finally, for arbitrarily high codimensions, we obtain a primary obstruction for fiber bundles over certain aspherical manifolds based on a new variant of the primary multipartitioned manifold index theorem, see Section 4.2. In Section 4.3, we demonstrate that, for codimensions greater than two, there cannot exist a general secondary multipartitioned manifold index theorem that is naively analogous to the partitioned manifold index theorem.

\section{Organization of the thesis}

In Chapter 1 we exhibit some prerequisites for the following chapters. In particular, we review the spectral picture of K-theory, discuss Yu's localization algebras and establish some technical conventions pertaining to the comparison of Riemannian metrics of psc on non-compact manifolds.

Chapter 2, which is the core part of this thesis, develops our secondary index theory for metrics of partial positive scalar curvature. In particular, this includes the product formulas, the secondary partitioned manifold index theorem and the coarse APS-index theorem. In Chapter 3, we discuss applications of the theory to closed manifolds and then how to use them to construct interesting examples on non-compact manifolds. Chapters 2 and 3 grew out of the author's article [Zei16] published in the Journal of Topology.

Finally, in Chapter 4, we discuss our applications to primary and secondary obstructions via submanifolds. The primary multi-partitioned manifold index theorem, its consequences on fiber bundles, and the primary codimension one result have been previously made available on the arXiv [Zei15]. 



\section{Preliminaries}

This chapter serves as a technical preparation for the rest of this thesis to recall requisite facts and set up the notation. We discuss the spectral picture of K-theory, elements of spin geometry, concordance of Riemannian metrics on non-compact manifolds, and Yu's localization algebras. Proofs in this chapter are usually abridged or replaced with references to the relevant literature.

\subsection{K-theory of $\mathrm{C}^{*}$-algebras}

\subsubsection{Graded $\mathrm{C}^{*}$-algebras}

We use the approach to K-theory for graded $\mathrm{C}^{*}$-algebras due to Trout [Tro00] following the exposition in the lecture notes [HG04].

Unless stated otherwise, we work with Real $\mathrm{C}^{*}$-algebras by default. However, the reader may "complexify" everything simply by ignoring the Real structure. A Real $\mathrm{C}^{*}$-algebra is a complex $\mathrm{C}^{*}$-algebra $A$ together with an involutive conjugate-linear *-automorphism $A \rightarrow A, a \mapsto \bar{a}$. We require *-homomorphisms $\varphi: A \rightarrow B$ between Real $\mathrm{C}^{*}$-algebras to preserve the Real structure, that is, $\varphi(\bar{a})=\overline{\varphi(a)}$ for all $a \in A$.

A grading on a (Real) $\mathrm{C}^{*}$-algebra $A$ is a Real *-automorphism $\alpha: A \rightarrow A$ such that $\alpha^{2}=\mathrm{id}$. A $\mathrm{C}^{*}$-algebra together with a grading is called a graded $\mathrm{C}^{*}$-algebra. Alternatively, a grading may be viewed as a direct sum decomposition $A=A^{(0)} \oplus A^{(1)}$ into selfadjoint Real subspaces such that $A^{(i)} A^{(j)} \subseteq A^{(i+j)}$, where $A^{(i)}$ is the $(-1)^{i}$ eigenspace of $\alpha, i \in \mathbb{Z}_{2}$. All $*$-homomorphisms $\varphi: A \rightarrow B$ between graded $\mathrm{C}^{*}$-algebras will be assumed to preserve the grading in the sense that $\varphi \circ \alpha=\alpha \circ \varphi$. Any $\mathrm{C}^{*}$-algebra can be trivially graded by setting $\alpha=\mathrm{id}$.

The Real $\mathrm{C}^{*}$-algebra of continuous functions on the real line which vanish at infinity admits a grading defined by the reflection map $f \mapsto(x \mapsto f(-x))$. We will denote this graded Real $\mathrm{C}^{*}$-algebra by $\mathcal{S}$.

Let $\mathcal{H}=\mathcal{H}^{(0)} \oplus \mathcal{H}^{(1)}$ be a fixed graded Real Hilbert space, where $\mathcal{H}^{(0)}=\mathcal{H}^{(1)}$ is countably infinite-dimensional. Let $\mathbb{K}$ denote the Real $\mathrm{C}^{*}$-algebra of compact operators on $\mathcal{H}$, graded by the decomposition into diagonal and off-diagonal matrices. Such a grading is known as a standard even grading.

Given two graded $C^{*}$-algebras $A$ and $B$, we denote their maximal graded tensor product by $A \widehat{\otimes} B$. We will always use maximal tensor products unless specified otherwise.

A central feature of this thesis is the use of Clifford algebras. See [ABS64; LM89] for general references. We use the following notation. The Clifford algebra $\mathrm{Cl}_{n, m}$ is the Real $\mathrm{C}^{*}$-algebra generated by real, odd generators $\left\{e_{1}, \ldots, e_{n}, \varepsilon_{1}, \ldots, \varepsilon_{m}\right\}$ subject to the relations $e_{i} e_{j}+e_{j} e_{i}=-2 \delta_{i j}, \varepsilon_{k} \varepsilon_{l}+\varepsilon_{l} \varepsilon_{k}=+2 \delta_{k l}, e_{i} \varepsilon_{k}+\varepsilon_{k} e_{i}=0, e_{i}^{*}=-e_{i}$, $\varepsilon_{k}^{*}=\varepsilon_{k}$. As shorthands we denote $\mathrm{Cl}_{n, 0}$ by $\mathrm{Cl}_{n}$ and $\mathrm{Cl}_{0, n}$ by $\mathrm{Cl}_{n}^{*}$. There is a canonical 
isomorphism $\mathrm{Cl}_{n, m} \widehat{\otimes} \mathrm{Cl}_{n^{\prime}, m^{\prime}}=\mathrm{Cl}_{n+n^{\prime}, m+m^{\prime}}$. In cases where we do not use the Real structure, we will denote the Clifford algebras by $\mathbb{C l}_{n}$. Moreover, $\mathrm{Cl}_{n, n}$ is isomorphic to the matrix algebra $\mathrm{M}_{2^{n}}(\mathbf{C})$. Here " $\mathbf{C}$ " denotes the Real algebra $\mathbb{C}$ endowed with the standard complex conjugation. For $n>0$, the algebra $\mathrm{Cl}_{n, n}$ is endowed with a standard even grading using an identification $\mathrm{Cl}_{n, n} \cong \mathrm{M}_{2^{n}}(\mathbf{C})=\mathrm{M}_{2}\left(\mathrm{M}_{2^{n-1}}(\mathbf{C})\right)$. In particular, we have an isomorphism $\mathrm{Cl}_{n, n} \widehat{\otimes} \mathbb{K} \cong \mathbb{K}$ for all $n \geqslant 0$.

Let $A, B$ be graded Real $\mathrm{C}^{*}$-algebras. Let $[A, B]$ be the set of homotopy classes of $*$-homomorphisms $A \rightarrow B$ (with respect to homotopies preserving the given Real structure and grading). In other words, $[A, B]=\pi_{0}(\operatorname{Hom}(A, B))$, where $\operatorname{Hom}(A, B)$ denotes the space of $*$-homomorphisms $A \rightarrow B$ endowed with the point-norm topology. The homotopy class of a $*$-homomorphism $\varphi: A \rightarrow B$ will be denoted by $[\varphi]$.

Definition 1.1.1. Let $A$ be a graded Real $C^{*}$-algebra. For $n \geqslant 0$, we define the group

$$
\mathrm{K}_{n}(A):=\pi_{n}(\underline{\mathrm{K}}(A)),
$$

where $\underline{K}(A):=\operatorname{Hom}(\mathcal{S}, A \widehat{\otimes} \mathbb{K})$ with the zero map as base-point.

One can verify that the $n$-fold loop space of $\underline{\mathrm{K}}(A)$ is canonically homeomorphic to $\underline{\mathrm{K}}\left(\Sigma^{n} A\right)$. Here $\Sigma^{n} A$ denotes the $n$-fold suspension of $A$, that is, $\Sigma^{n} A=\mathcal{C}_{0}\left(\mathbb{R}^{n}\right) \widehat{\otimes} A$, where $\mathcal{C}_{0}\left(\mathbb{R}^{n}\right)$ is endowed with the trivial grading. In particular, we have $\mathrm{K}_{n}(A)=$ $\pi_{0}\left(\underline{\mathrm{K}}\left(\Sigma^{n} A\right)\right)=\mathrm{K}_{0}\left(\Sigma^{n} A\right)=\left[\mathcal{S}, \Sigma^{n} A \widehat{\otimes} \mathbb{K}\right]$.

The direct sum induces a map $\underline{\mathrm{K}}(A) \times \underline{\mathrm{K}}(A) \rightarrow \underline{\mathrm{K}}(A)$, taking a pair $(\phi, \psi) \in$ $\underline{\mathrm{K}}(A) \times \underline{\mathrm{K}}(A)$ to the composition $\mathcal{S} \stackrel{\phi \oplus \psi}{\rightarrow}(A \widehat{\otimes} \mathbb{K}) \oplus(A \widehat{\otimes} \mathbb{K})=A \widehat{\otimes}(\mathbb{K} \oplus \mathbb{K}) \subset A \widehat{\otimes} \mathbb{K}$, where we use an embedding $\mathbb{K} \oplus \mathbb{K} \subset \mathbb{K}$ coming from the diagonal embedding $\mathbb{K} \oplus \mathbb{K} \subset \mathrm{M}_{2}(\mathbb{K})$ and an even unitary isomorphism $\mathcal{H} \oplus \mathcal{H} \cong \mathcal{H}$. The choice of such a unitary does not matter up to homotopy. It can be shown that this defines a commutative H-group structure on $\underline{\mathrm{K}}(A)$, thereby turning $\mathrm{K}_{n}(A)=\pi_{n}(\underline{\mathrm{K}}(A))$ into an abelian group for all $n \geqslant 0$. By a general principle in homotopy theory, this agrees with the homotopy group structure on $\pi_{n}$ for $n \geqslant 1$. It is possible to turn $\underline{K}(A)$ into a spectrum so that its homotopy groups are precisely the K-theory groups we have just defined, see [Del+11]. Remark 1.1.2. Any graded $*$-homomorphism $\varphi: \mathcal{S} \rightarrow A$ defines an element $[\varphi]:=$ $\left[\varphi \widehat{\otimes} e_{11}\right] \in \mathrm{K}_{0}(A)$, where $e_{11}$ is an even rank 1 projection in $\mathbb{K}$.

\subsubsection{External Product}

There is a comultiplication $\triangle: \mathcal{S} \rightarrow \mathcal{S} \widehat{\otimes} \mathcal{S}, f \mapsto f(\mathrm{x} \widehat{\otimes} 1+1 \widehat{\otimes} \mathrm{x})$, given by the functional calculus of the unbounded multiplier $\mathrm{x} \widehat{\otimes} 1+1 \widehat{\otimes} \mathrm{x}$. The comultiplication $\triangle$ is coassociative and counital (with counit $\eta: \mathcal{S} \rightarrow \mathbf{C}, \eta(f)=f(0)$ ).

On the generators $\left\{\mathrm{e}^{-\mathrm{x}^{2}}, \mathrm{xe}^{-\mathrm{x}^{2}}\right\}$ of $\mathcal{S}$ the comultiplication satisfies

$$
\triangle\left(\mathrm{e}^{-\mathrm{x}^{2}}\right)=\mathrm{e}^{-\mathrm{x}^{2}} \widehat{\otimes} \mathrm{e}^{-\mathrm{x}^{2}}, \quad \triangle\left(\mathrm{xe}^{-\mathrm{x}^{2}}\right)=\mathrm{xe}^{-\mathrm{x}^{2}} \widehat{\otimes} \mathrm{e}^{-\mathrm{x}^{2}}+\mathrm{e}^{-\mathrm{x}^{2}} \widehat{\otimes} \mathrm{xe}^{-\mathrm{x}^{2}} .
$$

We now present an explicit construction of $\triangle$ avoiding the use of unbounded multipliers. We have a Banach space isomorphism $\mathcal{S} \widehat{\otimes} \mathcal{S} \cong \mathcal{C}_{0}(\mathbb{R}) \otimes \mathcal{C}_{0}(\mathbb{R}) \cong \mathcal{C}_{0}\left(\mathbb{R}^{2}\right)$, which is determined by $f \widehat{\otimes} g \mapsto((x, y) \mapsto f(x) g(y))$. Clearly this is not an algebra isomorphism 
since the graded tensor product algebra $\mathcal{S} \widehat{\otimes} \mathcal{S}$ is not commutative. However, we can easily graft the algebra structure of $\mathcal{S} \widehat{\otimes} \mathcal{S}$ onto $\mathcal{C}_{0}\left(\mathbb{R}^{2}\right)$. In order to do that, let $\alpha_{\mathrm{x}}, \alpha_{\mathrm{y}}: \mathcal{C}_{0}\left(\mathbb{R}^{2}\right) \rightarrow \mathcal{C}_{0}\left(\mathbb{R}^{2}\right)$ denote the reflection automorphisms $f \mapsto((x, y) \mapsto f(-x, y))$ and $f \mapsto((x, y) \mapsto f(x,-y))$, respectively. Define

$$
\mathcal{C}_{0}^{(i, j)}\left(\mathbb{R}^{2}\right)=\left\{f \in \mathcal{C}_{0}\left(\mathbb{R}^{2}\right) \mid \alpha_{\mathrm{x}}(f)=(-1)^{i} f \text { and } \alpha_{\mathrm{y}}(f)=(-1)^{j} f\right\} .
$$

Since $\alpha_{\mathrm{x}}$ and $\alpha_{\mathrm{y}}$ are commuting involutions, there is a direct sum decomposition,

$$
\mathcal{C}_{0}\left(\mathbb{R}^{2}\right)=\mathcal{C}_{0}^{(0,0)}\left(\mathbb{R}^{2}\right) \oplus \mathcal{C}_{0}^{(1,1)}\left(\mathbb{R}^{2}\right) \oplus \mathcal{C}_{0}^{(1,0)}\left(\mathbb{R}^{2}\right) \oplus \mathcal{C}_{0}^{(0,1)}\left(\mathbb{R}^{2}\right)
$$

This defines a $\left(\mathbb{Z}_{2} \oplus \mathbb{Z}_{2}\right)$-grading on $\mathcal{C}_{0}\left(\mathbb{R}^{2}\right)$ in the sense that $\mathcal{C}_{0}^{(i, j)}\left(\mathbb{R}^{2}\right) \cdot \mathcal{C}_{0}^{(k, l)}\left(\mathbb{R}^{2}\right) \subseteq$ $\mathcal{C}_{0}^{(i+k, j+l)}\left(\mathbb{R}^{2}\right)$. Considering the first two summands together as the even component and the latter two as the odd component defines an ordinary $\left(\mathbb{Z}_{2^{-}}\right)$grading on $\mathcal{C}_{0}\left(\mathbb{R}^{2}\right)$ (in other words, use the grading operator $\alpha_{\mathrm{x}} \circ \alpha_{\mathrm{y}}$ ).

Using the above decomposition, we define a new product and star operation on $\mathcal{C}_{0}\left(\mathbb{R}^{2}\right)$ by setting

$$
f \hat{\bullet} g:=(-1)^{j k} f \cdot g, \quad f^{*}=(-1)^{i j} \bar{f} \quad \text { for } f \in \mathcal{C}_{0}^{(i, j)}\left(\mathbb{R}^{2}\right), g \in \mathcal{C}_{0}^{(k, l)}\left(\mathbb{R}^{2}\right),
$$

and extending linearly (the dot - denotes the usual pointwise product of functions). These operations have been constructed in such a way that $f \widehat{\otimes} g \mapsto((x, y) \mapsto f(x) g(y))$ induces a graded $*$-isomorphism $\mathcal{S} \widehat{\otimes} \mathcal{S} \cong\left(\mathcal{C}_{0}\left(\mathbb{R}^{2}\right), \hat{\bullet}\right)$.

Now we aim to construct $\triangle$. Let $r: \mathbb{R}^{2} \rightarrow \mathbb{R}$ be the Euclidean norm, that is, $r(x, y)=\sqrt{x^{2}+y^{2}}$, and define the function

$$
\xi: \mathbb{R}^{2} \rightarrow \mathbb{R}, \quad \xi(x, y)= \begin{cases}\frac{x+y}{r(x, y)} & \text { if }(x, y) \neq(0,0) \\ 1 & \text { otherwise }\end{cases}
$$

Since $\xi$ is globally bounded and continuous everywhere except at $(0,0)$, the pointwise product $\xi \cdot g$ is an element of $\mathcal{C}_{0}\left(\mathbb{R}^{2}\right)$ for every $g \in \mathcal{C}_{0}\left(\mathbb{R}^{2}\right)$ which satisfies $g(0,0)=0$. We define linear maps,

$$
\begin{aligned}
\widetilde{\triangle}^{(0)}: \mathcal{S}^{(0)} & \rightarrow \mathcal{C}_{0}^{(0,0)}\left(\mathbb{R}^{2}\right) \subset \mathcal{C}_{0}\left(\mathbb{R}^{2}\right), \\
f & \mapsto f \circ r, \\
\widetilde{\triangle}^{(1)}: \mathcal{S}^{(1)} & \rightarrow \mathcal{C}_{0}^{(1,0)}\left(\mathbb{R}^{2}\right) \oplus \mathcal{C}_{0}^{(0,1)}\left(\mathbb{R}^{2}\right) \subset \mathcal{C}_{0}\left(\mathbb{R}^{2}\right), \\
f & \mapsto \xi \cdot(f \circ r),
\end{aligned}
$$

and let $\widetilde{\triangle}:=\widetilde{\triangle}^{(0)} \oplus \widetilde{\triangle}^{(1)}: \mathcal{S} \rightarrow \mathcal{C}_{0}\left(\mathbb{R}^{2}\right)$. Direct calculation shows that $\widetilde{\triangle}$ is a graded *-homomorphism $\mathcal{S} \rightarrow\left(\mathcal{C}_{0}\left(\mathbb{R}^{2}\right), \hat{\bullet}\right)$ which satisfies

$$
\widetilde{\triangle}\left(\mathrm{e}^{-\mathrm{x}^{2}}\right)=\mathrm{e}^{-\mathrm{x}^{2}-\mathrm{y}^{2}}, \quad \widetilde{\triangle}\left(\mathrm{xe}^{-\mathrm{x}^{2}}\right)=(\mathrm{x}+\mathrm{y}) \mathrm{e}^{-\mathrm{x}^{2}-\mathrm{y}^{2}} .
$$

As (1.1.4) agrees with (1.1.1) up to the identification $\mathcal{S} \widehat{\otimes} \mathcal{S} \cong\left(\mathcal{C}_{0}\left(\mathbb{R}^{2}\right), \hat{\bullet}\right)$, we conclude that $\widetilde{\triangle}$ is really an implementation of $\triangle$.

Let $\varepsilon>0$ and let $\mathcal{S}(-\varepsilon, \varepsilon)$ be the graded ideal in $\mathcal{S}$ consisting of those functions which vanish outside the interval $(-\varepsilon, \varepsilon)$. 
Corollary 1.1.3. The comultiplication $\triangle$ preserves $\mathcal{S}(-\varepsilon, \varepsilon)$ in the sense that

$$
\triangle(\mathcal{S}(-\varepsilon, \varepsilon)) \subseteq \mathcal{S}(-\varepsilon, \varepsilon) \widehat{\otimes} \mathcal{S}(-\varepsilon, \varepsilon)
$$

Proof. Since $\mathcal{S}(-\varepsilon, \varepsilon) \widehat{\otimes} \mathcal{S}(-\varepsilon, \varepsilon) \cong\left(\mathcal{C}_{0}\left((-\varepsilon, \varepsilon)^{2}\right)\right.$, $\left.\hat{\bullet}\right)$, this follows from the explicit construction of $\triangle$ given in (1.1.2) and (1.1.3)

We explicitly note the following elementary lemma and a direct corollary because both are essential to our discussion of secondary invariants in Subsection 2.2.2.

Lemma 1.1.4. The inclusion maps $\mathcal{S}(-r, r) \hookrightarrow \mathcal{S}$ and $\mathcal{S}(-r, r) \widehat{\otimes} \mathcal{S}(-r, r) \hookrightarrow \mathcal{S} \widehat{\otimes} \mathcal{S}$ are graded homotopy equivalences for all $r>0$.

Proof. Let $0<r^{\prime}<r$ and choose an odd, monotonically increasing homeomorphism $\vartheta:(-r, r) \rightarrow \mathbb{R}$ which satisfies $\vartheta(s)=s$ for all $s \in\left(-r^{\prime}, r^{\prime}\right)$. Then $\vartheta^{*}: \mathcal{S} \rightarrow \mathcal{S}(-r, r)$ is a graded $*$-homomorphism which is a graded homotopy inverse to $\iota: \mathcal{S}(-r, r) \hookrightarrow \mathcal{S}$. Indeed, the identity map on $\mathcal{S}(-r, r)$ is graded homotopic to a $*$-homomorphism $\phi: \mathcal{S}(-r, r) \rightarrow \mathcal{S}(-r, r)$ the range of which is contained in $\mathcal{S}\left(-r^{\prime}, r^{\prime}\right)$. The map $\vartheta^{*}$ acts as the identity on $\mathcal{S}\left(-r^{\prime}, r^{\prime}\right)$ and thus we have $\vartheta^{*} \circ \iota \circ \phi=\phi$. This implies $\vartheta^{*} \circ \iota \simeq \vartheta^{*} \circ \iota \circ \phi=\phi \simeq \mathrm{id}$, that is, $\vartheta^{*}$ is a homotopy left-inverse to $\iota$.

To find a right-inverse, fix again an odd and monotonically increasing homeomorphism $\gamma:(-1,1) \rightarrow \mathbb{R}$, and let $\widetilde{h}_{s}: \mathcal{S}(-1,1) \rightarrow \mathcal{S}(-1,1), s \in[1, \infty]$, be defined as

$$
\widetilde{h}_{s}(f)(t)= \begin{cases}f(s t) & |t| \leqslant \frac{1}{s} \\ 0 & |t|>\frac{1}{s}\end{cases}
$$

Then consider the homotopy of graded $*$-homomorphisms $h_{s}:=\left(\gamma^{-1}\right)^{*} \circ \widetilde{h}_{s} \circ \gamma^{*}: \mathcal{S} \rightarrow \mathcal{S}$, $s \in[1, \infty)$. Clearly, $h_{1}=$ id and the range of $h_{s}$ is contained in $\mathcal{S}\left(-r_{s}, r_{s}\right)$ for $s>1$ where $r_{s}=\gamma\left(\frac{1}{s}\right)$. Since $r_{s} \searrow 0$ as $s \rightarrow \infty$, we have that $h_{s}: \mathcal{S} \rightarrow \mathcal{S}\left(-r_{s}, r_{s}\right)$ is a homotopy right-inverse to the inclusion $\iota$ for sufficiently large $s$. Finally, to obtain a homotopy inverse to $\mathcal{S}(-r, r) \widehat{\otimes} \mathcal{S}(-r, r) \hookrightarrow \mathcal{S} \widehat{\otimes} \mathcal{S}$, just use $\vartheta^{*} \widehat{\otimes} \vartheta^{*}$.

Corollary 1.1.5. Let $\psi: \mathcal{S} \rightarrow \mathcal{S}(-r, r)$ be a graded $*$-homomorphism such that the composition with the inclusion $\mathcal{S}(-r, r) \hookrightarrow \mathcal{S}$ is graded homotopic to the identity on $\mathcal{S}$. Then the following diagram of *-homomorphisms commutes up to graded homotopy.

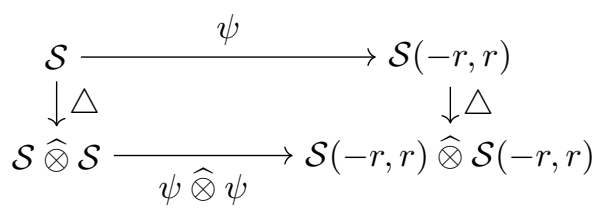

Proof. Follows immediately from Lemma 1.1.4 because $\triangle \circ \psi: \mathcal{S} \rightarrow \mathcal{S}(-r, r) \widehat{\otimes} \mathcal{S}(-r, r)$ and $\psi \widehat{\otimes} \psi \circ \triangle: \mathcal{S} \rightarrow \mathcal{S}(-r, r) \widehat{\otimes} \mathcal{S}(-r, r)$ are both homotopic to $\triangle: \mathcal{S} \rightarrow \mathcal{S} \widehat{\otimes} \mathcal{S}$ after composition with the inclusion $\mathcal{S}(-r, r) \widehat{\otimes} \mathcal{S}(-r, r) \hookrightarrow \mathcal{S} \widehat{\otimes} \mathcal{S}$. 
Definition 1.1.6 ([HG04, Section 1.7]). The external product

$$
\mathrm{K}_{n}(A) \otimes \mathrm{K}_{m}(B) \stackrel{\times}{\rightarrow} \mathrm{K}_{n+m}(A \widehat{\otimes} B)
$$

is induced by the map $\underline{\mathrm{K}}(A) \wedge \underline{\mathrm{K}}(B) \rightarrow \underline{\mathrm{K}}(A \widehat{\otimes} B)$, taking a pair $(\phi, \psi) \in \underline{\mathrm{K}}(A) \times \underline{\mathrm{K}}(B)$ to the composition $\mathcal{S} \stackrel{\leftrightarrow}{\rightarrow} \widehat{\otimes} \mathcal{S} \stackrel{\phi \widehat{\otimes} \psi}{\rightarrow} A \widehat{\otimes} \mathbb{K} \widehat{\otimes} B \widehat{\otimes} \mathbb{K} \cong A \widehat{\otimes} B \widehat{\otimes}(\mathbb{K} \widehat{\otimes} \mathbb{K}) \cong A \widehat{\otimes} B \widehat{\otimes} \mathbb{K}$.

Here we implicitly use a fixed isomorphism $\mathbb{K} \widehat{\otimes} \mathbb{K} \cong \mathbb{K}$ coming from an even unitary isomorphism $\mathcal{H} \widehat{\otimes} \mathcal{H} \cong \mathcal{H}$. As in the case of the direct sum, the choice of such an identification does not matter up to homotopy.

Remark 1.1.7. If $x=[\phi] \in \mathrm{K}_{0}(A)$ and $y=[\psi] \in \mathrm{K}_{0}(B)$ are represented by homomorphisms $\phi: \mathcal{S} \rightarrow A$ and $\psi: \mathcal{S} \rightarrow B$ as in Remark 1.1.2, then $x \times y$ is represented by $\phi \widehat{\otimes} \psi \circ \triangle: \mathcal{S} \rightarrow A \widehat{\otimes} B$ (the rank 1 projections take care of themselves because $e_{11} \widehat{\otimes} e_{11} \in \mathbb{K} \widehat{\otimes} \mathbb{K} \cong \mathbb{K}$ is again an even rank 1 projection).

\subsubsection{Bott periodicity}

In this subsection, we briefly sketch a variant of the "Dirac-dual-Dirac" approach to Bott periodicity using Clifford algebras. For more elaborations and proofs we refer to [HG04, Section 1.10], [Dum05, Lemma 4.3].

The dual Dirac element or Bott element is the class $b_{n} \in \mathrm{K}_{0}\left(\mathcal{C}_{0}\left(\mathbb{R}^{n}\right) \widehat{\otimes} \mathrm{Cl}_{n}^{*}\right)$ defined by the graded $*$-homomorphism

$$
\beta_{n}: \mathcal{S} \rightarrow \mathcal{C}_{0}\left(\mathbb{R}^{n}, \mathrm{Cl}_{n}^{*}\right), \quad \beta(f)=(v \mapsto f(v)),
$$

where " $f(v)$ " denotes the application of $f$ on $v \in \mathbb{R}^{n} \subseteq \mathrm{Cl}_{n}^{*}$ via the functional calculus in $\mathrm{Cl}_{n}^{*}$.

We will occasionally use asymptotic morphisms and ideas from E-theory. For general references see [GHT00; HG04]. This first surfaces in the following, where we use that an asymptotic morphism $\alpha: \mathcal{S} \widehat{\otimes} A \rightarrow B$ induces a map on K-theory $\alpha_{*}: \mathrm{K}_{*}(A) \rightarrow \mathrm{K}_{*}(B)$, see [HG04, Remark 1.11]. There is an asymptotic morphism $\alpha: \mathcal{S} \widehat{\otimes} \mathcal{C}_{0}\left(\mathbb{R}^{n}\right)-\rightarrow \mathbb{K} \widehat{\otimes} \mathrm{Cl}_{n}$, called the Dirac element. Indeed, $\alpha$ is defined using the Dirac operator $\mathfrak{D}_{\mathbb{R}^{n}}=\sum_{i=1}^{n} e_{i}$. $\frac{\partial}{\partial x_{i}}$ on $\mathrm{L}^{2}\left(\mathbb{R}^{n}, \mathrm{Cl}_{n}\right), \alpha_{t}(f \widehat{\otimes} g)=f\left(\frac{1}{t} \mathfrak{D}_{\mathbb{R}^{n}}\right) g$. It is the inverse of the dual Dirac element in the sense that the induced homomorphism $\alpha_{*}: \mathrm{K}_{0}\left(\mathcal{C}_{0}\left(\mathbb{R}^{n}\right) \widehat{\otimes} \mathrm{Cl}_{n}^{*}\right) \rightarrow \mathrm{K}_{0}(\mathbb{K})=\mathrm{K}_{0}(\mathbf{C})$ maps $b_{n}$ to the unit element $1 \in \mathrm{K}_{0}(\mathbf{C})$. A variant of Atiyah's rotation trick shows that the Bott map,

$$
\mathrm{K}_{0}(A) \rightarrow \mathrm{K}_{0}\left(A \widehat{\otimes} \mathcal{C}_{0}\left(\mathbb{R}^{n}\right) \widehat{\otimes} \mathrm{Cl}_{n}^{*}\right), \quad x \mapsto x \times b_{n},
$$

is an isomorphism for any graded $\mathrm{C}^{*}$-algebra $A$ and all $n \in \mathbb{N}$. In particular, there is a natural isomorphism

$$
\mathrm{K}_{0}\left(A \widehat{\otimes} \mathrm{Cl}_{n}\right) \cong \mathrm{K}_{0}\left(A \widehat{\otimes} \mathcal{C}_{0}\left(\mathbb{R}^{n}\right) \widehat{\otimes} \mathrm{Cl}_{n} \widehat{\otimes} \mathrm{Cl}_{n}^{*}\right) \cong \mathrm{K}_{0}\left(\Sigma^{n} A\right)=\mathrm{K}_{n}(A),
$$

where the second isomorphism follows because $\mathrm{Cl}_{n} \widehat{\otimes} \mathrm{Cl}_{n}^{*}=\mathrm{Cl}_{n, n} \cong \mathrm{M}_{2^{n}}(\mathbf{C})$ with a standard even grading. Moreover, $\mathrm{Cl}_{8} \cong \mathrm{M}_{16}(\mathbf{C})$ with a standard even grading, which together with (1.1.5) implies 8-fold periodicity of real K-theory. Similarly, in the complex case, we get 2 -fold periodicity because $\mathbb{C l}_{2} \cong \mathrm{M}_{2}(\mathbb{C})$. 


\subsubsection{Long exact sequences}

Let $0 \rightarrow I \rightarrow A \rightarrow A / I \rightarrow 0$ be a short exact sequence of graded $\mathrm{C}^{*}$-algebras. Then the induced map $\underline{\mathrm{K}}(A) \rightarrow \underline{\mathrm{K}}(A / I)$ is a Serre fibration with fiber $\underline{\mathrm{K}}(I)$. Thus, there is a long exact sequence of homotopy groups, which yields the natural long exact sequence in K-theory,

$$
\cdots \rightarrow \mathrm{K}_{n+1}(A / I) \stackrel{\partial}{\rightarrow} \mathrm{K}_{n}(I) \rightarrow \mathrm{K}_{n}(A) \rightarrow \mathrm{K}_{n}(A / I) \rightarrow \cdots \rightarrow \mathrm{K}_{0}(A / I) .
$$

Using Bott periodicity, one can also define K-theory for negative degrees and extend the exact sequence to the right. In fact, it then becomes the 24 term (respectively 6 term in the complex case) cyclic exact sequence.

If $B$ is another graded $\mathrm{C}^{*}$-algebra, then the sequence $0 \rightarrow I \widehat{\otimes} B \rightarrow A \widehat{\otimes} B \rightarrow$ $A / I \widehat{\otimes} B \rightarrow 0$ is exact, ${ }^{1}$ and the boundary map is compatible with the external product, that is, $\partial(x) \times y=\partial(x \times y)$ for $x \in \mathrm{K}_{*}(A / I), y \in \mathrm{K}_{*}(B)$.

In addition, there is a Mayer-Vietoris sequence for the K-theory of graded $\mathrm{C}^{*}$ algebras. Here we consider a graded $\mathrm{C}^{*}$-algebra $A$ and two closed two-sided graded ideals $I_{1}, I_{2} \unlhd A$ such that $I_{1}+I_{2}=A$. Then the long exact Mayer-Vietoris sequence reads as follows:

$$
\cdots \rightarrow \mathrm{K}_{n+1}(A) \stackrel{\partial_{\mathrm{MY}}}{\longrightarrow} \mathrm{K}_{n}\left(I_{1} \cap I_{2}\right) \rightarrow \mathrm{K}_{n}\left(I_{1}\right) \oplus \mathrm{K}_{n}\left(I_{2}\right) \rightarrow \mathrm{K}_{n}(A) \rightarrow \cdots
$$

To construct this sequence, consider the auxiliary $\mathrm{C}^{*}$-algebra $\Omega\left(A ; I_{1}, I_{2}\right)$ which consists of paths $f:[-\infty, \infty] \rightarrow A$ such that $f(-\infty) \in I_{1}$ and $f(+\infty) \in I_{2}$. The inclusion $I_{1} \cap I_{2} \hookrightarrow \Omega\left(A ; I_{1}, I_{2}\right)$ sending $b \in I_{1} \cap I_{2}$ to the constant path at $b$ is a K-isomorphism, see for instance [Sie12b, Lemma 3.1]. The short exact sequence $0 \rightarrow \Sigma A \rightarrow \Omega\left(A ; I_{1}, I_{2}\right) \stackrel{\mathrm{ev}_{\mp \infty}}{\longrightarrow}$ $I_{1} \oplus I_{2} \rightarrow 0$ induces a long exact sequence

$$
\cdots \rightarrow \mathrm{K}_{n}(\Sigma A) \rightarrow \mathrm{K}_{n}\left(\Omega\left(A ; I_{1}, I_{2}\right)\right) \rightarrow \mathrm{K}_{n}\left(I_{1} \oplus I_{2}\right) \rightarrow \mathrm{K}_{n-1}(\Sigma A) \rightarrow \cdots
$$

which is precisely of the form (1.1.6). The Mayer-Vietoris sequence is compatible with external products. In particular, there is a commutative diagram

$$
\begin{aligned}
& \mathrm{K}_{n+1}(A) \otimes \mathrm{K}_{m}(B) \stackrel{\partial_{\mathrm{MV}} \otimes \mathrm{id}}{\longrightarrow} \mathrm{K}_{n}\left(I_{1} \cap I_{2}\right) \otimes \mathrm{K}_{m}(B) \\
& \downarrow \times \\
& \mathrm{K}_{n+m+1}(A \widehat{\otimes} B) \stackrel{\partial_{\mathrm{MV}}}{\longrightarrow} \mathrm{K}_{n+m}\left(\left(I_{1} \widehat{\otimes} B\right) \cap\left(I_{2} \widehat{\otimes} B\right)\right) .
\end{aligned}
$$

Here we use the natural identification $\left(I_{1} \widehat{\otimes} B\right) \cap\left(I_{2} \widehat{\otimes} B\right)=\left(I_{1} \cap I_{2}\right) \widehat{\otimes} B$.

Remark 1.1.8. There is an alternative description of the Mayer-Vietoris boundary map in terms of the boundary map of some short exact sequence. Indeed, the inclusion induces an isomorphism $\iota: I_{1} / I_{1} \cap I_{2} \cong A / I_{2}$. Consider the boundary map $\partial_{1}: \mathrm{K}_{*}\left(I_{1} / I_{1} \cap I_{2}\right) \rightarrow \mathrm{K}_{*-1}\left(I_{1} \cap I_{2}\right)$ associated to $0 \rightarrow I_{1} \cap I_{2} \rightarrow I_{1} \rightarrow I_{1} / I_{1} \cap I_{2} \rightarrow 0$ and the canonical projection $\pi_{2}: A \rightarrow A / I_{2}$. Then

$$
\partial_{\mathrm{MV}}(x)=\partial_{1}\left(\iota^{-1} \circ \pi_{2}\right)_{*}(x) \in \mathrm{K}_{*-1}\left(I_{1} \cap I_{2}\right)
$$

for all $x \in \mathrm{K}_{*}(A)$.

\footnotetext{
${ }^{1}$ Recall that we use the maximal tensor product.
} 


\subsection{Spin geometry}

In this section, we briefly discuss essential facts from spin geometry. For a comprehensive introduction we refer the reader to [LM89].

\subsubsection{Spin structures}

All the manifolds and related structures (such as bundles over manifolds and their sections) we consider are smooth. Let $(X, g)$ be an oriented Riemannian $n$-manifold. Let $\mathrm{P}_{\mathrm{GL}^{+}}(\mathrm{T} X)$ and $\mathrm{P}_{\mathrm{SO}}(\mathrm{T} X)$ denote the oriented and the special orthogonal frame bundles, respectively. A spin structure on $X$ is a principal $\operatorname{Spin}(n)$-bundle $\mathrm{P}_{\text {Spin }}(\mathrm{T} X)$ together with a two-sheeted covering $\xi: \mathrm{P}_{\mathrm{Spin}}(\mathrm{T} X) \rightarrow \mathrm{P}_{\mathrm{SO}}(\mathrm{T} X)$ such that $\xi(p g)=\xi(p) \xi_{0}(g)$ for all $p \in \mathrm{P}_{\mathrm{Spin}}(\mathrm{T} X)$ and $g \in \operatorname{Spin}(n)$, where $\xi_{0}: \operatorname{Spin}(n) \rightarrow \mathrm{SO}(n)$ is the (connected, if $n \geqslant 2)$ two-sheeted covering of $\mathrm{SO}(n)$.

Spin structures are in one-to-one correspondence with two-sheeted coverings of $\mathrm{P}_{\mathrm{SO}}(\mathrm{T} X)$ which are non-trivial over the fibers (if $\left.n \geqslant 2\right)^{2}$. Let $g^{\prime}$ be some (other) Riemannian metric on $X$ and $\mathrm{P}_{\mathrm{SO}^{\prime}}(\mathrm{T} X)$ the corresponding special orthogonal frame bundle. Then we have inclusions $\mathrm{P}_{\mathrm{SO}}(\mathrm{T} X) \hookrightarrow \mathrm{P}_{\mathrm{GL}^{+}}(\mathrm{T} X) \hookleftarrow \mathrm{P}_{\mathrm{SO}^{\prime}}(\mathrm{T} X)$ which are both fiberwise homotopy equivalences. Therefore, every spin structure on $(X, g)$ induces a unique spin structure on $\left(X, g^{\prime}\right)$ and vice versa.

In view of the previous discussion, the concept of a spin structure on $X$ makes sense independently of the choice of the Riemannian metric $g$. A spin manifold is an oriented manifold together with a spin structure.

\subsubsection{The spinor Dirac operator}

Let $l: \operatorname{Spin}(n) \rightarrow \mathrm{GL}\left(\mathrm{Cl}_{n}\right)$ denote the representation by left multiplication of $\operatorname{Spin}(n)$ viewed as a subgroup of the multiplicative units of the Clifford algebra.

Let $X$ be a spin manifold. Then the $\mathrm{Cl}_{n}$-linear spinor bundle associated to a Riemannian metric $g$ is the associated bundle

$$
\Phi_{g}:=\mathrm{P}_{\text {Spin }}(\mathrm{T} X) \times{ }_{l} \mathrm{Cl}_{n}
$$

The right-multiplication action of $\mathrm{Cl}_{n}$ on itself turns $\phi_{g}$ into a bundle of free graded right- $\mathrm{Cl}_{n}$-modules of rank one. Moreover, the $\mathrm{Cl}_{n}$-valued inner product $\mathrm{Cl}_{n} \ni a, b \mapsto$ $(a \mid b):=a^{*} b$ on $\mathrm{Cl}_{n}$ induces a $\mathrm{Cl}_{n}$-valued fiberwise inner product on $\Phi_{g}$, thereby turning it into a bundle of graded Hilbert $\mathrm{Cl}_{n}$-modules. Its compactly supported smooth sections, denoted by $\Gamma_{\mathrm{c}}^{\infty}\left(X, \Phi_{g}\right)$, are also endowed with a $\mathrm{Cl}_{n}$-linear inner product:

$$
\langle s \mid t\rangle:=\int_{X}(s \mid t) \operatorname{vol}_{g}, \quad s, t \in \Gamma_{\mathrm{c}}^{\infty}\left(X, \Phi_{g}\right) .
$$

The space of $\mathrm{L}^{2}$-sections $\mathrm{L}^{2}\left(X, \Phi_{g}\right)$ of $\Phi_{g}$ is the completion of $\Gamma_{\mathrm{c}}^{\infty}\left(X, \Phi_{g}\right)$ with respect to this inner product; it is a graded Real Hilbert- $\mathrm{Cl}_{n}$-module.

There exists a unique grading-preserving connection $\nabla^{g}$ on $\Phi_{g}$ with the following properties for all $a \in \mathrm{Cl}_{n}, \xi, \eta \in \Gamma^{\infty}(X, \mathrm{~T} X), s, t \in \Gamma^{\infty}\left(X, \Phi_{g}\right)$ :

\footnotetext{
${ }^{2}$ For $n=1$, a spin structure is simply a two-fold covering of $X$.
} 
(i) $\nabla_{\xi}^{g}(s a)=\left(\nabla_{\xi}^{g} s\right) a$,

(ii) $\nabla_{\xi}^{g}(c(\eta) s)=c\left(\nabla_{\xi}^{\mathrm{LC}, g} \eta\right) s+c(\eta) \nabla_{\xi}^{g} s$, where $\nabla^{\mathrm{LC}, g}$ is the Levi-Civita connection,

(iii) $\xi \cdot\langle s \mid t\rangle=\left\langle\nabla_{\xi}^{g} s \mid t\right\rangle+\left\langle s \mid \nabla_{\xi}^{g} t\right\rangle$.

The spinor Dirac operator associated to $g$ is the following composition:

$$
\mathscr{D}_{g}: \Gamma^{\infty}\left(X, \mathscr{\phi}_{g}\right) \stackrel{\nabla^{g}}{\rightarrow} \Gamma^{\infty}\left(X, \mathrm{~T}^{*} X \otimes \overleftarrow{\phi}_{g}\right) \stackrel{c}{\rightarrow} \Gamma^{\infty}\left(X, \oiint_{g}\right),
$$

where $c$ denotes the Clifford multiplication action of the cotangent bundle.

\subsection{Metric considerations on non-compact manifolds}

Although the notion of a spin structure does not depend on the Riemannian metric, in our subsequent applications of large-scale index theory to positive scalar curvature problems on non-compact manifolds some global restrictions on the Riemannian metric will become necessary. Instead of looking at all Riemannian metrics on a given manifold at once, we will only study classes of Riemannian metrics at a time which are uniformly controlled by a fixed reference metric. Moreover, if the manifold has a boundary, we need to make some restrictions to ensure that the metrics we consider are well-behaved near the boundary. The conventions we set up in this section will be used implicitly throughout this thesis.

\subsubsection{Admissible metrics on manifolds with boundary}

Let $X$ be a manifold with boundary. A collar of $\partial X$ is an embedding $c: \partial X \times(-1,0] \rightarrow$ $X$ such that the following diagram commutes:

$$
\partial X \times(-1,0] \stackrel{c}{\longrightarrow} \underset{\iota_{\iota_{0}}^{X}}{\longrightarrow} \underset{\partial X .}{\uparrow}
$$

Collar neighborhoods always exist, see for example [Hir94, Chapter 4].

We say that a Riemannian metric $g$ on $X$ has product structure near $\partial X$ with respect to the collar $c$ if there exists $\varepsilon>0$ such that $c^{*} g\left\lceil\partial X \times(-\varepsilon, 0]=\partial g \oplus \mathrm{d} t^{2}\right.$, where $\partial g$ denotes the restriction of $g$ to $\partial X$.

Given a Riemannian metric $g$ on $X$, we denote the induced distance function by $d_{g}: X \times X \rightarrow \mathbb{R}_{\geqslant 0}$. If $g$ has product structure near the boundary with respect to some collar, then there exists a $\delta>0$ such that for all $x, y \in \partial X$ and $t, s \in(-\delta, 0]$ with $d_{g}(c(x, t), c(y, s))<\delta$, we have

$$
d_{g}(c(x, t), c(y, s))=\sqrt{d_{\partial g}(x, y)^{2}+|t-s|^{2}} .
$$


In particular, $d_{g}(x, y)=d_{\partial g}(x, y)$ for all $x, y \in \partial X$ with $d_{g}(x, y)<\delta$. Moreover, it is always true that $d_{g}(x, y) \leqslant d_{\partial g}(x, y)$ for all $x, y \in \partial X$.

Usually, the distance functions on manifolds we consider are induced by Riemannian metrics. However, when working with manifolds that are obtained by attaching a cylindrical end to a manifold with boundary, we will modify the distance function slightly (see Subsection 1.3.3 below). To treat both cases in a uniform way, we introduce the following auxilliary concept. We say that a metric $d_{X}$ (in the sense of a distance function) on $X$ is admissible if it generates the manifold topology, is proper, and is of product structure near the boundary in the sense that there exists $\delta>0$ such that for all $x, y \in \partial X$ and $t, s \in(-\delta, 0]$,

$$
d_{X}(c(x, t), c(y, s))<\delta \quad \Rightarrow \quad d_{X}(c(x, t), c(y, s))=\sqrt{d_{X}(x, y)^{2}+|t-s|^{2}}
$$

for some fixed collar neighborhood $c$. In addition, we require an admissible distance function to be uniformly dominated by a Riemannian metric, that is, there exists a Riemannian metric $g$ with id: $\left(X, d_{g}\right) \rightarrow\left(X, d_{X}\right)$ uniformly continuous. A distance function induced by a Riemannian metric with product structure near the boundary is always admissible by the discussion in the previous paragraph.

Definition 1.3.1. Let $X$ be a manifold, possibly with boundary and, if so, with an implicitly fixed collar. Fix an admissible distance function $d_{X}$. We define $\mathcal{R}\left(X, d_{X}\right)$ to be the set of all Riemannian metrics $g$ on $X$ such that

(i) it has product structure near $\partial X$ with respect to the fixed collar,

(ii) the identity map $\left(X, d_{g}\right) \rightarrow\left(X, d_{X}\right)$ is uniformly continuous.

The set $\mathcal{R}\left(X, d_{X}\right)$ only depends on the uniform equivalence class of $d_{X}$. If the choice of the uniform equivalence class of the distance function $d_{X}$ is implicit in the context, we will simply write $\mathcal{R}(X)$. Since the distance function associated to a Riemannian metric is a length metric, it follows that the identity $\left(X, d_{g}\right) \rightarrow\left(X, d_{X}\right)$ is a large-scale Lipschitz map for all $g \in \mathcal{R}\left(X, d_{X}\right)$, see [Roe03, Lemma 1.10].

Remark 1.3.2. Given $\left(X, d_{X}\right)$ and $\left(Y, d_{Y}\right)$, we endow $X \times Y$ with the Euclidean product metric

$$
d_{X \times Y}\left(\left(x_{1}, y_{1}\right),\left(x_{2}, y_{2}\right)\right):=\sqrt{d_{X}\left(x_{1}, x_{2}\right)^{2}+d_{Y}\left(y_{1}, y_{2}\right)^{2}} .
$$

For many arguments it is crucial that one can use convex combinations to interpolate between any two Riemannian metrics. Given the importance of this fact, we include the following elementary lemma to show that this is still possible in our present context.

Lemma 1.3.3. Let $g_{0}, g_{1} \in \mathcal{R}\left(X, d_{X}\right), t_{0}<t_{1} \in \mathbb{R}$, and let $\chi: \mathbb{R} \rightarrow[0,1]$ be a smooth function with $\chi(t)=0$ for $t \leqslant t_{0}$ and $\chi(t)=1$ for $t \geqslant t_{1}$. Then the Riemannian metric on $X \times \mathbb{R}$ defined as follows,

$$
h_{(x, t)}:=\left((1-\chi(t))\left(g_{0}\right)_{x}+\chi(t)\left(g_{1}\right)_{x}\right) \oplus \mathrm{d} t^{2}
$$

belongs to $\mathcal{R}\left(X \times \mathbb{R}, d_{X \times \mathbb{R}}\right)$. 
Proof. Let $\varepsilon>0$. Fix $\varepsilon>\delta>0$ such that for all $t<s \in \mathbb{R}$ with $s-t<\delta$, we have $\chi(\tau) \in(1 / 4,1]$ for all $\tau \in[t, s]$ or $\chi(\tau) \in[0,3 / 4)$ for all $\tau \in[t, s]$.

Now let $a=(x, t), b=(y, s) \in X \times \mathbb{R}$ with $t<s$ and $d_{h}(a, b)<\delta$. Note that $\mathrm{pr}_{2}: X \times \mathbb{R} \rightarrow \mathbb{R}$ is distance non-increasing. Hence $s-t<\delta$. Without loss of generality, we may assume that $\chi(\tau) \in(1 / 4,1]$ for all $\tau \in[t, s]$. Let $\gamma:[0,1] \rightarrow X \times \mathbb{R}$ be a piecewise smooth path with $\gamma(0)=a$ and $\gamma(1)=b$ such that its length with respect to the Riemannian metric $h$ is less than $d_{h}(a, b)+\varepsilon$. We may assume that $\left(\operatorname{pr}_{2} \circ \gamma\right)([0,1]) \subseteq[t, s]$ and hence $\left(\chi \circ \operatorname{pr}_{2} \circ \gamma\right)([0,1]) \subseteq(1 / 4,1]$. We may compute as follows:

$$
\begin{aligned}
2 \varepsilon>\operatorname{length}_{h}(\gamma)=\int_{0}^{1} \sqrt{h(\dot{\gamma}, \dot{\gamma})} \geqslant \int_{0}^{1} \sqrt{\left(\chi \circ \operatorname{pr}_{2} \circ \gamma\right) g_{1}\left(\operatorname{pr}_{1} \dot{\gamma}, \operatorname{pr}_{1} \dot{\gamma}\right)} \\
\quad>\frac{1}{2} \int_{0}^{1} \sqrt{g_{1}\left(\operatorname{pr}_{1} \dot{\gamma}, \operatorname{pr}_{1} \dot{\gamma}\right)}=\frac{1}{2} \operatorname{length}_{g_{1}}\left(\operatorname{pr}_{1} \circ \gamma\right) \geqslant \frac{1}{2} d_{g_{1}}(x, y) .
\end{aligned}
$$

We deduce $d_{g_{1}}(x, y)<4 \varepsilon$. If $\chi(\tau) \in\left[0, \frac{3}{4}\right)$ for all $\tau \in[t, s]$, then an analogous argument shows $d_{g_{0}}(x, y)<4 \varepsilon$.

Hence for all $a=(x, t), b=(y, s) \in X \times \mathbb{R}$ with $d_{h}(a, b)<\delta$, we always have $|t-s|<\varepsilon$ and $d_{g_{0}}(x, y)<4 \epsilon$ or $d_{g_{1}}(x, y)<4 \varepsilon$. Since the identity map $\left(X, d_{g_{i}}\right) \rightarrow(X, d)$ is uniformly continuous for $i \in\{0,1\}$, this shows that the identity $\left(X \times \mathbb{R}, d_{h}\right) \rightarrow$ $\left(X \times \mathbb{R}, d_{X \times \mathbb{R}}\right)$ is uniformly continuous.

\subsubsection{Concordance of positive scalar curvature metrics}

We now introduce some notation which will be convenient for discussing positive scalar curvature phenomena.

Definition 1.3.4. Let $X$ be a manifold together with an admissible distance function and let $Z \subseteq X$ be some subset. We say that $g \in \mathcal{R}(X)$ has uniformly positive scalar curvature ("upsc") outside $Z$ if

$$
\inf \left\{\operatorname{scal}_{g}(x) \mid x \in X \backslash Z\right\}>0 .
$$

We let $\mathcal{R}_{Z}^{+}(X)$ denote set of all $g \in \mathcal{R}(X)$ such that $g$ has upsc outside $Z$.

Furthermore, we set $\mathcal{R}^{+}(X):=\mathcal{R}_{\emptyset}^{+}(X)$.

In the following, we suppose that $X$ is endowed with an isometric (with respect to $d_{X}$ ), proper, and free action of a discrete group $\Gamma$ by diffeomorphisms. If $Z \subseteq X$ is a $\Gamma$-invariant subset, we will denote the subset of $\Gamma$-invariant metrics in $\mathcal{R}_{Z}^{+}(X)$ by $\mathcal{R}_{Z}^{+}(X)^{\Gamma}$.

Definition 1.3.5. Let $g_{0}, g_{1} \in \mathcal{R}_{Z}^{+}(X)^{\Gamma}$. We call $g_{0}$ and $g_{1}$ concordant relative to $Z$ if there exists a Riemannian metric $h \in \mathcal{R}_{Z \times \mathbb{R}}^{+}(X \times \mathbb{R})^{\Gamma}$ such that

(i) $h\left\lceil X \times(-\infty, 0]=g_{0} \oplus \mathrm{d} t^{2}\right.$,

(ii) $h \uparrow X \times[1, \infty)=g_{1} \oplus \mathrm{d} t^{2}$. 
If $Z=\emptyset, g_{0}$ and $g_{1}$ are called concordant.

Remark 1.3.6. Lemma 1.3.3 shows that if $g_{0}, g_{1} \in \mathcal{R}_{Z}^{+}(X)^{\Gamma}$ are equal outside $Z$, then they are concordant relative to $Z$. In particular, any two Riemannian metrics in $\mathcal{R}(X)^{\Gamma}$ are concordant relative to $X$.

\subsubsection{Attaching a cylindrical end}

Let $X$ be a manifold with boundary endowed with a proper metric $d_{X}$. Let $d_{\partial X}$ denote the restriction of $d_{X}$ to $\partial X$ and endow $\partial X \times \mathbb{R}_{\geqslant 0}$ with the Euclidean product metric $d_{\partial X \times \mathbb{R} \geqslant 0}$.

Definition 1.3.7. Let

$$
X_{\infty}:=X \cup_{\partial X}\left(\partial X \times \mathbb{R}_{\geqslant 0}\right),
$$

where we identify $\partial X$ with the subset $\partial X \times\{0\}$ of $\partial X \times \mathbb{R}_{\geqslant 0}$. We endow $X_{\infty}$ with the metric $d_{X_{\infty}}$ obtained by gluing $d_{X}$ and $d_{\partial X \times \mathbb{R}_{\geqslant 0}}$ along $\partial X$. More precisely, $d_{X_{\infty}} \uparrow X:=d_{X}, d_{X_{\infty}} \uparrow\left(\partial X \times \mathbb{R}_{\geqslant 0}\right):=d_{\partial X \times \mathbb{R}_{\geqslant 0}}$, and for $x \in X$ and $y \in \partial X \times \mathbb{R}_{\geqslant 0}$ we set

$$
d_{X_{\infty}}(x, y):=\inf _{z \in \partial X}\left(d_{X}(x, z)+d_{\partial X \times \mathbb{R} \geqslant 0}(z, y)\right) .
$$

Remark 1.3.8. One can endow $X_{\infty}$ with a smooth structure via the (implicitly fixed) collar of $X$, compare [Hir94, Section 8.2]. Using this smooth structure, any Riemmanian metric $g \in \mathcal{R}\left(X, d_{X}\right)$ extends uniquely to a Riemannian metric $g_{\infty} \in \mathcal{R}\left(X_{\infty}, d_{X_{\infty}}\right)$ on $X_{\infty}$ which restricts to $g$ on $X$ and to the product metric $\partial g \oplus \mathrm{d} t^{2}$ on $\partial X \times \mathbb{R}_{\geqslant 0}$.

Remark 1.3.9. If $X$ is endowed with a group action as in the previous subsection, then this induces a group action on $X_{\infty}$ with the same properties.

\subsection{Yu's localization algebras and K-homology}

$\mathrm{Yu}$ [Yu97] has introduced the localization algebra $\mathrm{C}^{*} \mathrm{~L}(X)$ to provide an alternative model for the analytic $\mathrm{K}$-homology of a proper metric space $X$. Indeed, there is an isomorphism $\mathrm{K}_{*}(X) \stackrel{\cong}{\rightarrow} \mathrm{K}_{*}\left(\mathrm{C}^{*} \mathrm{~L}(X)\right)$, called the "local index map", under which the coarse index map Ind: $\mathrm{K}_{*}(X) \rightarrow \mathrm{K}_{*}\left(\mathrm{C}^{*}(X)\right)$ can be implemented by a canonical *-homomorphism; see Theorem 1.4 .20 for a precise statement. Here $\mathrm{C}^{*}(X)$ denotes the Roe algebra of $X$.

\subsubsection{Localization algebras}

In this subsection, we review the definition of the localization algebras and introduce a $\mathrm{Cl}_{n}$-linear version of them.

Let $X$ be a proper metric space endowed with an isometric, free and proper action of a countable discrete group $\Gamma$.

A $\Gamma$-equivariant $X$-module, or simply $(X, \Gamma)$-module, is a Hilbert space $H$ together with a $*$-representation $\rho: \mathcal{C}_{0}(X) \rightarrow \mathbb{B}(H)$ and a unitary representation $U: \Gamma \rightarrow \mathscr{U}(H)$ 
such that $U_{\gamma} \rho(f) U_{\gamma}^{*}=\rho(\gamma \cdot f)$ for all $\gamma \in \Gamma$ and $f \in \mathcal{C}_{0}(X)$, where $(\gamma \cdot f)(x)=f\left(\gamma^{-1} x\right)$. If $\Gamma$ is the trivial group we refer to it just as an $X$-module.

$\mathrm{A} \mathrm{Cl}_{n}$-linear $\Gamma$-equivariant $X$-module, or simply $\left(X, \Gamma, \mathrm{Cl}_{n}\right)$-module, is defined analogously, but we replace the Hilbert space $H$ by a graded Hilbert $\mathrm{Cl}_{n}$-module $\mathfrak{H}$ and require that the representations are by even bounded $\mathrm{Cl}_{n}$-linear operators. ${ }^{3}$

An $(X, \Gamma)$-module $H$ is called ample if the representation $\rho$ is non-degenerate and $\rho(f)$ is not a compact operator for any non-zero $f \in \mathcal{C}_{0}(X)$. In the $\mathrm{Cl}_{n}$-linear setting, we call an $\left(X, \Gamma, \mathrm{Cl}_{n}\right)$-module ample if it is isomorphic to $H \widehat{\otimes} \mathrm{Cl}_{n}$, where $H$ is an ample $(X, \Gamma)$-module.

Henceforth, we will simplify the notation and suppress the representation $\rho: \mathcal{C}_{0}(X) \rightarrow$ $\mathbb{B}(H)$ by identifying $f \in \mathcal{C}_{0}(X)$ with $\rho(f) \in \mathbb{B}(H)$.

In the remainder of this subsection, $X, Y, Z$ will be proper metric spaces endowed with free and proper $\Gamma$-actions, and $\mathfrak{H}_{W}$ will be ample $\left(W, \Gamma, \mathrm{Cl}_{n}\right)$-modules for $W \in\{X, Y, Z\}$. Given two Hilbert $\mathrm{Cl}_{n}$-modules $E, F$, we write $\mathbb{B}(E, F)$ for the bounded $\mathrm{Cl}_{n}$-linear operators $E \rightarrow F$. If $E$ is a graded Hilbert $\mathrm{Cl}_{n}$-module, then $\mathbb{B}(E)=\mathbb{B}(E, E)$ is a graded $\mathrm{C}^{*}$-algebra.

Definition 1.4.1. The support of $\xi \in \mathfrak{H}_{X}$ is the subset $\operatorname{supp}(\xi) \subseteq X$ such that $x \notin \operatorname{supp}(\xi)$ if and only if there exists $f \in \mathcal{C}_{0}(X)$ such that $f \xi=0$ and $f(x) \neq 0$.

Definition 1.4.2. The support of $T \in \mathbb{B}\left(\mathfrak{H}_{X}, \mathfrak{H}_{Y}\right)$ is the $\operatorname{subset} \operatorname{supp}(T) \subseteq Y \times X$ such that $(y, x) \notin \operatorname{supp}(T)$ if and only if there exist $f \in \mathcal{C}_{0}(X)$ and $g \in \mathcal{C}_{0}(Y)$ such that $g T f=0$ and $f(x) \neq 0 \neq g(y)$.

Definition 1.4.3. The propagation of $T \in \mathbb{B}\left(\mathfrak{H}_{X}\right)$ is

$$
\operatorname{prop}(T):=\sup \left\{d_{X}(y, x) \mid(y, x) \in \operatorname{supp}(T)\right\} .
$$

Definition 1.4.4. Let $A \subset X$ be a subset. We say that $T \in \mathbb{B}\left(\mathfrak{H}_{X}\right)$ is supported near $A$ if there exists $R \geqslant 0$ such that $\operatorname{supp}(T) \subseteq \mathcal{U}_{R}(A) \times \mathcal{U}_{R}(A)$, where $\mathcal{U}_{R}(A)$ denotes the open $R$-neighborhood of $A$ in $X$.

Remark 1.4.5 (Calculus of supports, see [HR00, Lemma 6.3.6]). For subsets $A \subseteq X$, $B \subseteq Y \times X$ and $C \subseteq Z \times Y$, we write

$$
\begin{gathered}
B \cdot A:=\{y \mid \exists x \in X:(y, x) \in B, x \in A\} \subseteq Y \\
C \circ B:=\{(z, x) \mid \exists y \in Y:(z, y) \in B,(y, x) \in A\} \subseteq Z \times X, \\
\left.B^{*}:=\{(x, y) \mid(y, x) \in A\}\right\} \subseteq X \times Y .
\end{gathered}
$$

Let $T \in \mathbb{B}\left(\mathfrak{H}_{X}, \mathfrak{H}_{Y}\right)$. Then

$$
\operatorname{supp}\left(T^{*}\right)=\operatorname{supp}(T)^{*}
$$

Furthermore, $\operatorname{supp}(T)$ is the smallest closed subset $B \subseteq Y \times X$ such that $\operatorname{supp}(T \xi) \subseteq$ $B \cdot \operatorname{supp}(\xi)$ for all $\xi \in \mathfrak{H}_{X}$ with $\operatorname{supp}(\xi)$ compact. Now suppose that $T$ is proper in

\footnotetext{
${ }^{3}$ In the case of $\mathrm{Cl}_{n}$, an operator is bounded and $\mathrm{Cl}_{n}$-linear iff it is an adjointable Hilbert-module map.
} 
the sense that $\operatorname{supp}(T \xi)$ is compact for all $\xi \in \mathfrak{H}_{X}$ with $\operatorname{supp}(\xi)$ compact. Then for all $S \in \mathbb{B}\left(\mathfrak{H}_{Y}, \mathfrak{H}_{Z}\right)$ the following inclusion holds:

$$
\operatorname{supp}(S \circ T) \subseteq \operatorname{supp}(S) \circ \operatorname{supp}(T) .
$$

Since operators of finite propagation over a proper metric space are always proper, it follows that

$$
\operatorname{prop}(S \circ T) \leqslant \operatorname{prop}(S)+\operatorname{prop}(T)
$$

for all bounded operators $T, S \in \mathbb{B}\left(\mathfrak{H}_{X}\right)$.

Before introducing the Roe and localization algebras, we review some more preliminary concepts of operators on $X$-modules.

Definition 1.4.6. Let $T \in \mathbb{B}\left(\mathfrak{H}_{X}\right)$. We say that $T$ is

(i) locally compact if $f T \in \mathbb{K}\left(\mathfrak{H}_{X}\right) \ni T f$ for all $f \in \mathcal{C}_{0}(X)$,

(ii) pseudo-local if $f T-T f \in \mathbb{K}\left(\mathfrak{H}_{X}\right)$ for all $f \in \mathcal{C}_{0}(X)$.

Definition 1.4.7 (Roe and localization algebras). Let $A \subseteq B \subseteq X$ be $\Gamma$-invariant subsets and let $B$ be closed.

(1) Let $\mathbf{C}\left[X ; \mathrm{Cl}_{n}\right]^{\Gamma}$ denote the set of all $\Gamma$-equivariant, bounded, $\mathrm{Cl}_{n}$-linear operators on $\mathfrak{H}_{X}$ which are locally compact and of finite propagation. The $\mathrm{Cl}_{n}$-linear $\Gamma$-equivariant Roe algebra, denoted by $\mathrm{C}_{\Gamma}^{*}\left(X ; \mathrm{Cl}_{n}\right)$, is the closure of $\mathbf{C}\left[X ; \mathrm{Cl}_{n}\right]^{\Gamma}$ in the norm topology.

(2) Let $\mathbf{C}\left[A \subset X ; \mathrm{Cl}_{n}\right]^{\Gamma}$ denote the set of all $T \in \mathbf{C}\left[X ; \mathrm{Cl}_{n}\right]^{\Gamma}$ such that $T$ is supported near $A$. Its closure in the norm topology is denoted by $\mathrm{C}_{\Gamma}^{*}\left(A \subset X ; \mathrm{Cl}_{n}\right)$.

(3) Let $\mathbf{C L}\left[X ; \mathrm{Cl}_{n}\right]^{\Gamma}$ denote the set of all bounded and uniformly continuous functions $L:[1, \infty) \rightarrow \mathbf{C}\left[X ; \mathrm{Cl}_{n}\right]^{\Gamma}$ such that the propagation of $L(t)$ tends to zero as $t \rightarrow \infty$. The $\mathrm{Cl}_{n}$-linear $\Gamma$-equivariant localization algebra, denoted by $\mathrm{C}_{\Gamma}^{*} \mathrm{~L}(X)$, is the completion of $\mathrm{CL}\left[X ; \mathrm{Cl}_{n}\right]^{\Gamma}$ with respect to the uniform-norm topology.

(4) Let $\mathbf{C L}\left[B \subset X ; \mathrm{Cl}_{n}\right]^{\Gamma}$ be the set of those $L \in \mathbf{C L}\left[X ; \mathrm{Cl}_{n}\right]^{\Gamma}$ such that

$$
\operatorname{supp}(L(t)) \subseteq \mathcal{U}_{S(r)}(B) \times \mathcal{U}_{S(r)}(B)
$$

for some function $S:[1, \infty) \rightarrow \mathbb{R}_{\geqslant 0}$ with $S(r) \rightarrow 0$ as $r \rightarrow \infty$.

Let $\mathrm{C}_{\Gamma}^{*} \mathrm{~L}\left(B \subset X ; \mathrm{Cl}_{n}\right)$ be the closure of $\mathbf{C L}\left[B \subset X ; \mathrm{Cl}_{n}\right]^{\Gamma}$.

(5) Evaluation at 1 yields a surjective $*$-homomorphism

$$
\mathrm{ev}_{1}: \mathbf{C L}\left[X ; \mathrm{Cl}_{n}\right]^{\Gamma} \rightarrow \mathbf{C}\left[X ; \mathrm{Cl}_{n}\right]^{\Gamma} .
$$

Let $\mathrm{CL}_{0}\left[X ; \mathrm{Cl}_{n}\right]^{\Gamma}$ be its kernel. More generally, let $\mathbf{C L}_{A}\left[X ; \mathrm{Cl}_{n}\right]^{\Gamma}$ be the preimage of $\mathbf{C}\left[A \subset X ; \mathrm{Cl}_{n}\right]^{\Gamma}$ under ev $\mathrm{ev}_{1}$ Let $\mathrm{C}_{\Gamma}^{*} \mathrm{~L}_{0}\left(X ; \mathrm{Cl}_{n}\right)$ and $\mathrm{C}_{\Gamma}^{*} \mathrm{~L}_{A}\left(X ; \mathrm{Cl}_{n}\right)$ be the closures of $\mathrm{CL}_{0}\left[X ; \mathrm{Cl}_{n}\right]^{\Gamma}$ and $\mathrm{CL}_{A}\left[X ; \mathrm{Cl}_{n}\right]^{\Gamma}$, respectively. 
(6) Let $\mathbf{C L}_{A}\left[B \subset X ; \mathrm{Cl}_{n}\right]^{\Gamma}$ be the intersection $\mathbf{C L}_{A}\left[X ; \mathrm{Cl}_{n}\right]^{\Gamma} \cap \mathbf{C L}\left[B \subset X ; \mathrm{Cl}_{n}\right]^{\Gamma}$. Let $\mathrm{C}_{\Gamma}^{*} \mathrm{~L}_{A}\left(B \subset X ; \mathrm{Cl}_{n}\right)$ be its closure.

Remark 1.4.8. The relations (1.4.1) and (1.4.3) imply that $\mathbf{C}\left[A \subset X ; \mathrm{Cl}_{n}\right]^{\Gamma}$ and $\mathrm{CL}_{A}\left[B \subset X ; \mathrm{Cl}_{n}\right]^{\Gamma}$ are graded $*$-subalgebras of the graded $\mathrm{C}^{*}$-algebras $\mathbb{B}\left(\mathfrak{H}_{X}\right)$ and $\mathcal{C}_{\mathrm{b}}\left([1, \infty), \mathbb{B}\left(\mathfrak{H}_{X}\right)\right)$, respectively. Hence $\mathrm{C}_{\Gamma}^{*}\left(A \subset X ; \mathrm{Cl}_{n}\right)$ and $\mathrm{C}_{\Gamma}^{*} \mathrm{~L}_{A}\left(B \subset X ; \mathrm{Cl}_{n}\right)$ are graded $\mathrm{C}^{*}$-algebras.

Remark 1.4.9. The algebras $\mathbf{C}\left[A \subset X ; \mathrm{Cl}_{n}\right]^{\Gamma}, \mathbf{C L}_{A}\left[B \subset X ; \mathrm{Cl}_{n}\right]^{\Gamma}, \mathrm{C}_{\Gamma}^{*}\left(A \subset X ; \mathrm{Cl}_{n}\right)$, and $\mathrm{C}_{\Gamma}^{*} \mathrm{~L}_{A}\left(B \subset X ; \mathrm{Cl}_{n}\right)$ include all others as special cases for appropriate choices of the subsets $A$ and $B$.

Remark 1.4.10 (Notation). If we wish to emphasize which $\left(X, \Gamma, \mathrm{Cl}_{n}\right)$-module is used, we will write (for example) $\mathrm{C}_{\Gamma}^{*} \mathrm{~L}\left(X, \mathfrak{H}_{X} ; \mathrm{Cl}_{n}\right)$. However, since the K-theory of these algebras does not depend on the choice of the ample module (see Subsection 1.4.2), we will usually not do so. There are also simpler versions of the Roe and localization algebras where there is no group action or no Clifford algebra present (take $\Gamma=1$ or $n=0)$. We denote these variants by dropping the group or the Clifford algebra in our notation, for instance $\mathrm{C}^{*} \mathrm{~L}\left(X ; \mathrm{Cl}_{n}\right), \mathrm{C}_{\Gamma}^{*} \mathrm{~L}(X), \mathrm{C}^{*} \mathrm{~L}(X), \mathrm{C}^{*} \mathrm{~L}(X, H), \ldots$

Lemma 1.4.11. The subsets $\mathbf{C}\left[A \subset X ; \mathrm{Cl}_{n}\right]^{\Gamma}$ and $\mathrm{C}_{\Gamma}^{*}\left(A \subset X ; \mathrm{Cl}_{n}\right)$ are selfadjoint ideals in $\mathbf{C}\left[X ; \mathrm{Cl}_{n}\right]^{\Gamma}$ and $\mathrm{C}_{\Gamma}^{*}\left(X ; \mathrm{Cl}_{n}\right)$, respectively. Similary, $\mathrm{CL}_{A}\left[B \subset X ; \mathrm{Cl}_{n}\right]^{\Gamma}$ and $\mathrm{C}_{\Gamma}^{*} \mathrm{~L}_{A}\left(B \subset X ; \mathrm{Cl}_{n}\right)$ are selfadjoint ideals in $\mathrm{CL}\left[X ; \mathrm{Cl}_{n}\right]^{\Gamma}$ and $\mathrm{C}_{\Gamma}^{*} \mathrm{~L}\left(X ; \mathrm{Cl}_{n}\right)$, respectively.

Proof. Let $T \in \mathbf{C}\left[A \subset X ; \mathrm{Cl}_{n}\right]^{\Gamma}$ and $S \in \mathbf{C}\left[X ; \mathrm{Cl}_{n}\right]^{\Gamma}$. Then $T^{*} \in \mathbf{C}\left[A \subset X ; \mathrm{Cl}_{n}\right]^{\Gamma}$ by (1.4.1). Let $R>0$ and $R^{\prime}>0$ be such that $\operatorname{supp}(T) \subseteq \mathcal{U}_{R}(A) \times \mathcal{U}_{R}(A)$ and $\operatorname{prop}(S)<R^{\prime}$. We deduce from (1.4.3) that

$$
\operatorname{supp}(S T) \subseteq \operatorname{supp}(S) \circ\left(\mathcal{U}_{R}(A) \times \mathcal{U}_{R}(A)\right) \subseteq \mathcal{U}_{R+R^{\prime}}(A) \times \mathcal{U}_{R}(A) .
$$

Hence $S T \in \mathbf{C}\left[A \subset X ; \mathrm{Cl}_{n}\right]^{\Gamma}$. Moreover, $T S=\left(S^{*} T^{*}\right)^{*} \in \mathbf{C}\left[A \subset X ; \mathrm{Cl}_{n}\right]^{\Gamma}$. This shows that $\mathbf{C}\left[A \subset X ; \mathrm{Cl}_{n}\right]^{\Gamma}$ is a selfadjoint ideal in $\mathbf{C}\left[X ; \mathrm{Cl}_{n}\right]^{\Gamma}$. An analogous argument using (1.4.1) and (1.4.4) shows that $\mathrm{CL}_{A}\left[B \subset X ; \mathrm{Cl}_{n}\right]^{\Gamma}$ is a selfadjoint ideal in $\mathrm{CL}\left[X ; \mathrm{Cl}_{n}\right]^{\Gamma}$. The remaining statements follow by passing to the completions.

Remark 1.4.12. Evaluation at 1 extends to a surjective $*$-homomorphism

$$
\mathrm{ev}_{1}: \mathrm{C}_{\Gamma}^{*} \mathrm{~L}\left(X ; \mathrm{Cl}_{n}\right) \rightarrow \mathrm{C}_{\Gamma}^{*}\left(X ; \mathrm{Cl}_{n}\right) .
$$

An approximation argument shows:

$$
\mathrm{C}_{\Gamma}^{*} \mathrm{~L}_{A}\left(X ; \mathrm{Cl}_{n}\right)=\mathrm{ev}_{1}^{-1}\left(\mathrm{C}_{\Gamma}^{*}\left(A \subset X ; \mathrm{Cl}_{n}\right)\right) .
$$

Remark 1.4.13. Due to the favourable properties of closed ideals in $\mathrm{C}^{*}$-algebras, it is usually straightforward to evaluate intersections of ideals in the Roe- and localization algebras. The following calculation illustrates what we mean: 
We will show that $\mathrm{C}_{\Gamma}^{*} \mathrm{~L}_{A}\left(B \subset X ; \mathrm{Cl}_{n}\right)=\mathrm{C}_{\Gamma}^{*} \mathrm{~L}_{A}\left(X ; \mathrm{Cl}_{n}\right) \cap \mathrm{C}_{\Gamma}^{*} \mathrm{~L}\left(B \subset X ; \mathrm{Cl}_{n}\right)$. It follows directly from the definitions that $\mathrm{C}_{\Gamma}^{*} \mathrm{~L}_{A}\left(B \subset X ; \mathrm{Cl}_{n}\right) \subseteq \mathrm{C}_{\Gamma}^{*} \mathrm{~L}_{A}\left(X ; \mathrm{Cl}_{n}\right) \cap$ $\mathrm{C}_{\Gamma}^{*} \mathrm{~L}\left(B \subset X ; \mathrm{Cl}_{n}\right)$. Conversely,

$$
\begin{aligned}
\mathrm{C}_{\Gamma}^{*} \mathrm{~L}_{A}\left(X ; \mathrm{Cl}_{n}\right) \cap \mathrm{C}_{\Gamma}^{*} \mathrm{~L}\left(B \subset X ; \mathrm{Cl}_{n}\right) & =\left(\mathrm{C}_{\Gamma}^{*} \mathrm{~L}_{A}\left(X ; \mathrm{Cl}_{n}\right)\right)\left(\mathrm{C}_{\Gamma}^{*} \mathrm{~L}\left(B \subset X ; \mathrm{Cl}_{n}\right)\right) \\
= & \overline{\left(\mathrm{CL}_{A}\left[X ; \mathrm{Cl}_{n}\right]^{\Gamma}\right)\left(\mathrm{CL}\left[B \subset X ; \mathrm{Cl}_{n}\right]^{\Gamma}\right)} \subseteq \overline{\mathrm{CL}_{A}\left[X ; \mathrm{Cl}_{n}\right]^{\Gamma} \cap \mathbf{C L}\left[B \subset X ; \mathrm{Cl}_{n}\right]^{\Gamma}} \\
= & \overline{\mathrm{CL}_{A}\left[B \subset X ; \mathrm{Cl}_{n}\right]^{\Gamma}}=\mathrm{C}_{\Gamma}^{*} \mathrm{~L}_{A}\left(B \subset X ; \mathrm{Cl}_{n}\right) .
\end{aligned}
$$

Here we have used that the intersection of two closed ideals in a $\mathrm{C}^{*}$-algebra equals its product (see for example [Mur90, p. 82]).

The most important feature of the ideals such as $\mathrm{C}^{*} \mathrm{~L}(B \subset X)$ is that their K-theory agrees with the K-theory of the corresponding algebra associated with the subspace $B$, see Lemma 1.4.18 below.

In addition, we will sometimes need the structure algebra $\mathrm{D}_{\Gamma}^{*}(X)$, which is defined to be the $\mathrm{C}^{*}$-algebra generated by all $\Gamma$-equivariant, pseudo-local operators of finite propagation. We also have the corresponding localization algebra version, $\mathrm{D}_{\Gamma}^{*} \mathrm{~L}(X)$, see also [QR10].

\subsubsection{Functoriality of localization algebras}

The K-theory of the localization algebra is functorial with respect to uniformly continuous and coarse maps. In the context of Roe algebras (and similarly, in the Paschke duality picture of K-homology), the induced map on the algebra level is given by conjugation with an isometry which in a suitable sense "covers" the original map on the space level. In this section, we review these ideas in a way that is adapted to the $\mathrm{Cl}_{n}$-linear setting and all the different versions of the localization algebra introduced in the previous subsection. However, we will only sketch the proofs in this subsection since they are straightforward modifications of constructions that appear in the standard literature on analytic K-homology and coarse index theory. Indeed, detailed treatments for the Roe algebra can be found in [HR00, Chapters 5 and 6], [Roe96; Sie12b] and for the localization algebras in [Yu97; QR10].

Let $X$ and $Y$ be proper metric spaces endowed with free and proper $\Gamma$-actions. A proper map $f: X \rightarrow Y$ is uniformly continuous and coarse ("ucc") if and only if there exists a non-decreasing function $S: \mathbb{R}_{\geqslant 0} \rightarrow \mathbb{R}_{\geqslant 0}$ which is continuous at 0 with $S(0)=0$ such that $d_{Y}\left(f\left(x_{1}\right), f\left(x_{2}\right)\right)<S\left(d_{X}\left(x_{1}, x_{2}\right)\right)$ for all $x_{1}, x_{2} \in X$.

Definition 1.4.14. Let $f: X \rightarrow Y$ be a $\Gamma$-equivariant ucc map. Fix an $\left(X, \Gamma, \mathrm{Cl}_{n}\right)$ module $\mathfrak{H}_{X}$ and a $\left(Y, \Gamma, \mathrm{Cl}_{n}\right)$-module $\mathfrak{H}_{Y}$. We say that a uniformly continuous family of $\Gamma$-equivariant $\mathrm{Cl}_{n}$-linear even isometries $V(t): \mathfrak{H}_{X} \rightarrow \mathfrak{H}_{Y}, t \in[1, \infty)$, covers $f$ if $\sup \{d(y, f(x)) \mid(y, x) \in \operatorname{supp}(V(t))\} \rightarrow 0$ as $t \rightarrow \infty$.

In [Yu97; QR10] functoriality is discussed only with respect to proper Lipschitz maps, but the construction generalizes directly to the case of ucc maps.

From now on we fix an ample $\left(X, \Gamma, \mathrm{Cl}_{n}\right)$-module $\mathfrak{H}_{X}$ and an ample $\left(Y, \Gamma, \mathrm{Cl}_{n}\right)$-module $\mathfrak{H}_{Y}$. 
Lemma 1.4.15. Let $f: X \rightarrow Y$ be a $\Gamma$-equivariant coarse map. Then there exists a family of isometries $V(t): \mathfrak{H}_{X} \rightarrow \mathfrak{H}_{Y} \oplus \mathfrak{H}_{Y}, t \in[1, \infty)$, that covers $f$ as in Definition 1.4.14.

Proof. Since an ample $\left(X, \Gamma, \mathrm{Cl}_{n}\right)$-module $\mathfrak{H}_{X}$ is by definition isomorphic to $H_{X} \widehat{\otimes} \mathrm{Cl}_{n}$ for an ample $(X, \Gamma)$-module $H_{X}$, we can ignore the Clifford algebra coefficients without loss of generality. A $\Gamma$-equivariant variant of the proof of [HR00, Proposition 6.3.12] and [HR00, Claim 6.3.14] shows that for every uniformly bounded countable open cover $\left(U_{l}\right)_{l \in \mathbb{N}}$ of $Y$, there exists an isometry $\tilde{V}: H_{X} \rightarrow H_{Y}$ such that

$$
\operatorname{supp}(\tilde{V}) \subseteq \bigcup_{l \in \mathbb{N}} U_{l} \times f^{-1}\left(U_{l}\right)
$$

By choosing the open cover so that each $U_{l}$ has sufficiently small diameter, we deduce that for each $k \in \mathbb{N}$, there exists an isometry $\tilde{V}_{k}: H_{X} \rightarrow H_{Y}$ such that

$$
\sup \left\{d(y, f(x)) \mid(y, x) \in \operatorname{supp}\left(\tilde{V}_{k}\right)\right\} \leqslant \frac{1}{k} .
$$

The proof will be complete if we manage to find a family of isometries that suitably interpolates between the $\tilde{V}_{k}$. This is achieved by the construction from [Yu97, p. 313]: Define for $k \in \mathbb{N}, t \in[0,1], \xi \in H_{X}$ :

$$
V(k+t) \xi:=\left(\begin{array}{cc}
\cos \left(\frac{\pi t}{2}\right) & \sin \left(\frac{\pi t}{2}\right) \\
-\sin \left(\frac{\pi t}{2}\right) & \cos \left(\frac{\pi t}{2}\right)
\end{array}\right)\left(\begin{array}{cc}
\tilde{V}_{k} & 0 \\
0 & \tilde{V}_{k+1}
\end{array}\right)\left(\begin{array}{cc}
\cos \left(\frac{\pi t}{2}\right) & -\sin \left(\frac{\pi t}{2}\right) \\
\sin \left(\frac{\pi t}{2}\right) & \cos \left(\frac{\pi t}{2}\right)
\end{array}\right)\left(\begin{array}{l}
\xi \\
0
\end{array}\right) .
$$

Proposition 1.4.16. Let $f: X \rightarrow Y$ be a $\Gamma$-equivariant ucc map and $V(t): \mathfrak{H}_{X} \rightarrow$ $\mathfrak{H}_{Y} \oplus \mathfrak{H}_{Y}$ be a family of isometries that covers $f$. Then conjugation by $V$ induces a *-homomorphism

$$
\mathrm{Ad}_{V}: \mathrm{C}_{\Gamma}^{*} \mathrm{~L}\left(X ; \mathrm{Cl}_{n}\right) \rightarrow \mathrm{M}_{2}\left(\mathrm{C}_{\Gamma}^{*} \mathrm{~L}\left(Y ; \mathrm{Cl}_{n}\right)\right), \quad L \mapsto\left(t \mapsto V(t) L(t) V(t)^{*}\right) .
$$

The induced map $\left(\mathrm{Ad}_{V}\right)_{*}: \mathrm{K}_{*}\left(\mathrm{C}_{\Gamma}^{*} \mathrm{~L}\left(X ; \mathrm{Cl}_{n}\right)\right) \rightarrow \mathrm{K}_{*}\left(\mathrm{C}_{\Gamma}^{*} \mathrm{~L}\left(Y ; \mathrm{Cl}_{n}\right)\right)$ does not depend on the choice of the family of isometries $V$ covering $f$. In particular, the $\mathrm{K}$-theory of $\mathrm{C}_{\Gamma}^{*} \mathrm{~L}\left(X ; \mathrm{Cl}_{n}\right)$ does not depend on the choice of the ample $\left(X, \Gamma, \mathrm{Cl}_{n}\right)$-module up to canonical isomorphism.

Proof. We only show that the propagation of $\operatorname{Ad}_{V}(L)$ tends to zero as $t \rightarrow \infty$ for all $L \in \mathbf{C L}\left[X ; \mathrm{Cl}_{n}\right]^{\Gamma}$. The rest of the proof proceeds along the same lines as in $[\mathrm{Yu} 97$; QR10], see also [HR00, Chapters 5-6]. Remark 1.4.5 shows

$$
\operatorname{supp}\left(\operatorname{Ad}_{V}(L)(t)\right) \subseteq \operatorname{supp}(V(t)) \circ \operatorname{supp}(L(t)) \circ \operatorname{supp}(V(t))^{*} .
$$

Let $\varepsilon>0$. Find $\delta>0$ and $R \geqslant 1$ such that:

$$
\begin{aligned}
\forall x^{\prime}, x \in X: & d_{X}\left(x^{\prime}, x\right)<\delta \Longrightarrow d_{Y}\left(f\left(x^{\prime}\right), f(x)\right)<\varepsilon, \\
\forall t \geqslant R: & \operatorname{prop}(L(t))<\delta, \\
\forall t \geqslant R: & \sup \left\{d_{Y}(y, f(x)) \mid(y, x) \in \operatorname{supp}(V(t))\right\}<\varepsilon .
\end{aligned}
$$


Fix $t \geqslant R$ and let $\left(y^{\prime}, y\right) \in \operatorname{supp}\left(\operatorname{Ad}_{V}(L)(t)\right)$. By (1.4.5), there exists $x^{\prime}, x \in X$ with $\left(y^{\prime}, x^{\prime}\right) \in \operatorname{supp}(V(t)),\left(x^{\prime}, x\right) \in \operatorname{supp}(L(t)),(y, x) \in \operatorname{supp}(V(t))$. We deduce

$$
d_{Y}\left(y^{\prime}, y\right) \leqslant d_{Y}\left(y^{\prime}, f\left(x^{\prime}\right)\right)+d_{Y}\left(f\left(x^{\prime}\right), f(x)\right)+d_{Y}(f(x), y)<3 \varepsilon .
$$

Therefore, $f$ induces a well-defined map on the K-theory of the localization algebras. We will incorporate this in our notation by writing

$$
f_{*}:=\left(\mathrm{Ad}_{V}\right)_{*}: \mathrm{K}_{*}\left(\mathrm{C}_{\Gamma}^{*} \mathrm{~L}\left(X ; \mathrm{Cl}_{n}\right)\right) \rightarrow \mathrm{K}_{*}\left(\mathrm{C}_{\Gamma}^{*} \mathrm{~L}\left(Y ; \mathrm{Cl}_{n}\right)\right) .
$$

If $A \subseteq X$ and $B \subseteq Y$ are $\Gamma$-invariant subsets with $f(A) \subseteq B$, then $\operatorname{Ad}_{V}$ takes $\mathrm{C}_{\Gamma}^{*} \mathrm{~L}_{A}\left(X ; \mathrm{Cl}_{n}\right)$ to $\mathrm{C}_{\Gamma}^{*} \mathrm{~L}_{B}\left(Y ; \mathrm{Cl}_{n}\right)$. Hence the same procedure yields a well-defined induced map

$$
f_{*}: \mathrm{K}_{*}\left(\mathrm{C}_{\Gamma}^{*} \mathrm{~L}_{A}\left(X ; \mathrm{Cl}_{n}\right)\right) \rightarrow \mathrm{K}_{*}\left(\mathrm{C}_{\Gamma}^{*} \mathrm{~L}_{B}\left(Y ; \mathrm{Cl}_{n}\right)\right)
$$

and shows that $\mathrm{K}_{*}\left(\mathrm{C}_{\Gamma}^{*} \mathrm{~L}_{A}\left(X ; \mathrm{Cl}_{n}\right)\right)$ does not depend on the choice of the ample $\left(X, \Gamma, \mathrm{Cl}_{n}\right)$-module up to canonical isomorphism. Moreover, for a family of isometries $V(t)$ which covers a ucc map $f$, the isometry $V(1)$ covers $f$ in the coarse sense, and hence the maps on K-theory of the localization algebras are compatible with the induced maps on the K-theory of the Roe algebras as they are defined in [HR00, Chapter 6].

Remark 1.4.17. Let $H$ be an ample $(X, \Gamma)$-module. Using the ample $\left(X, \Gamma, \mathrm{Cl}_{n}\right)$-module $\mathfrak{H}=H \widehat{\otimes} \mathrm{Cl}_{n}$, one can directly verify that $\mathrm{C}_{\Gamma}^{*} \mathrm{~L}\left(X, \mathfrak{H} ; \mathrm{Cl}_{n}\right)=\mathrm{C}_{\Gamma}^{*} \mathrm{~L}(X, H) \widehat{\otimes} \mathrm{Cl}_{n}$. This means that we have a canonical identification on the level of K-theory,

$$
\mathrm{K}_{0}\left(\mathrm{C}_{\Gamma}^{*} \mathrm{~L}\left(X ; \mathrm{Cl}_{n}\right)\right)=\mathrm{K}_{0}\left(\mathrm{C}_{\Gamma}^{*} \mathrm{~L}(X) \widehat{\otimes} \mathrm{Cl}_{n}\right)=\mathrm{K}_{n}\left(\mathrm{C}_{\Gamma}^{*} \mathrm{~L}(X)\right),
$$

where the latter identification is due to the Bott map from Subsection 1.1.3.

Lemma 1.4.18. Let $B \subseteq X$ be a closed $\Gamma$-invariant subset. Let $V(t): H_{B} \rightarrow H_{X}$ be a family of isometries which covers the inclusion $B \hookrightarrow X$, where $H_{Y}$ is an ample $(Y, \Gamma)$-module for $Y \in\{X, B\}$. Then conjugation by $V$ determines $*$-homomorphisms as follows:

$$
\begin{aligned}
\operatorname{Ad}_{V}: \mathrm{C}_{\Gamma}^{*} \mathrm{~L}_{0}(B) & \rightarrow \mathrm{C}_{\Gamma}^{*} \mathrm{~L}_{0}(B \subset X), \\
\operatorname{Ad}_{V}: \mathrm{C}_{\Gamma}^{*} \mathrm{~L}(B) & \rightarrow \mathrm{C}_{\Gamma}^{*} \mathrm{~L}(B \subset X), \\
\operatorname{Ad}_{V}: \mathrm{C}_{\Gamma}^{*}(B) & \rightarrow \mathrm{C}_{\Gamma}^{*}(B \subset X) .
\end{aligned}
$$

These *-homomorphisms induce (canonical) isomorphisms on K-theory.

Moreover, if $A \subseteq B$ is a further $\Gamma$-invariant subset, then $\operatorname{Ad}_{V}: \mathrm{C}_{\Gamma}^{*} \mathrm{~L}_{A}(B) \rightarrow$ $\mathrm{C}_{\Gamma}^{*} \mathrm{~L}_{A}(B \subset X)$ induces a canonical isomorphism on $\mathrm{K}$-theory.

Proof. This has been proved for the Roe algebra in [HRY93]. For the localization algebra $\mathrm{C}^{*} \mathrm{~L}(B)$, this has been directly established in [Yu97] in the special case of metric simplicial complexes. However, one can reduce the general case for $\mathrm{C}_{\Gamma}^{*} \mathrm{~L}(B)$ to the corresponding statement in (equivariant) K-homology. Indeed, if we suppose that $H_{B}$ and $H_{X}$ are both very ample ${ }^{4}$, it is shown in [QR10] that we have isomorphisms,

$$
\mathrm{K}_{*+1}\left(\mathrm{D}_{\Gamma}^{*}(B) / \mathrm{C}_{\Gamma}^{*}(B)\right) \stackrel{\cong}{\leftrightarrows} \mathrm{K}_{*+1}\left(\mathrm{D}_{\Gamma}^{*} \mathrm{~L}(B) / \mathrm{C}_{\Gamma}^{*} \mathrm{~L}(B)\right) \stackrel{\cong}{\rightrightarrows} \mathrm{K}_{*}\left(\mathrm{C}_{\Gamma}^{*} \mathrm{~L}(B)\right) .
$$

\footnotetext{
${ }^{4}$ That is, each is a countably infinite direct sum of an ample module.
} 
Similarly, we obtain isomorphisms

$$
\begin{aligned}
& \mathrm{K}_{*+1}\left(\mathrm{D}_{\Gamma}^{*}(B \subset X) / \mathrm{C}_{\Gamma}^{*}(B \subset X)\right) \stackrel{\cong}{\leftarrow} \mathrm{K}_{*+1}\left(\mathrm{D}_{\Gamma}^{*} \mathrm{~L}(B \subset X) / \mathrm{C}_{\Gamma}^{*} \mathrm{~L}(B \subset X)\right) \\
& \stackrel{\cong}{\rightrightarrows} \mathrm{K}_{*}\left(\mathrm{C}_{\Gamma}^{*} \mathrm{~L}(B \subset X)\right) \text {, }
\end{aligned}
$$

where the ideals $\mathrm{D}_{\Gamma}^{*}(B \subset X)$ and $\mathrm{D}_{\Gamma}^{*} \mathrm{~L}(B \subset X)$ are defined analogously as their counterparts in the setting of Roe algebras, however, with the additional condition that all operators should be locally compact on the complement of $B$. Furthermore, we observe that $\mathrm{Ad}_{V}$ intertwines these two sequences of isomorphisms, so it would suffice if

$$
\operatorname{Ad}_{V_{1}}: \mathrm{D}_{\Gamma}^{*}(B) / \mathrm{C}_{\Gamma}^{*}(B) \rightarrow \mathrm{D}_{\Gamma}^{*}(B \subset X) / \mathrm{C}_{\Gamma}^{*}(B \subset X)
$$

induced isomorphisms on K-theory. This, however, is just the corresponding statement in the Paschke duality picture of K-homology, which is proved in [HR00, Chapter 5], see also [Sie12b, Proposition 3.8].

Having established the isomorphisms for $\mathrm{C}_{\Gamma}^{*}(B)$ and $\mathrm{C}_{\Gamma}^{*} \mathrm{~L}(B)$, one can deduce from the Five Lemma that the map $\operatorname{Ad}_{V}: \mathrm{C}_{\Gamma}^{*} \mathrm{~L}_{0}(B) \rightarrow \mathrm{C}_{\Gamma}^{*} \mathrm{~L}_{0}(B \subset X)$ must also be an isomorphism on K-theory. Finally, the last statement follows similarly from a Five Lemma argument by considering the short exact sequence

$$
0 \rightarrow \mathrm{C}_{\Gamma}^{*} \mathrm{~L}_{0}(B \subset X) \rightarrow \mathrm{C}_{\Gamma}^{*} \mathrm{~L}_{A}(B \subset X) \rightarrow \mathrm{C}_{\Gamma}^{*}(A \subset X) \rightarrow 0 .
$$

\subsubsection{Analytic K-homology}

We will use the notation $\mathrm{K}_{*}^{\Gamma}(X)$ to denote the (equivariant) analytic K-homology of a locally compact space $X$ endowed with a proper and free group action. In terms of bivariant $\mathrm{K}$-theory for $\mathrm{C}^{*}$-algebras, this group is defined as $\mathrm{K}_{*}^{\Gamma}(X):=\mathrm{KK}_{*}^{\Gamma}\left(\mathcal{C}_{0}(X), \mathbf{C}\right)$. For a standard treatment of analytic K-homology, we refer to [HR00].

Since we have assumed the group action to be free and proper, we have an identification

$$
\mathrm{K}_{*}^{\Gamma}(X) \cong \mathrm{K}_{*}(\Gamma \backslash X),
$$

see for instance [HR00, Lemma 12.5.4] and [Sie12b, Subsection 2.4].

To avoid confusion, we note that, from a topological point of view, analytic Khomology on locally compact spaces is a locally finite homology theory; in particular, it is functorial with respect to proper continuous maps. Indeed, as can for instance be seen in the Paschke duality picture [HR00, Chapter 5], the group $\mathrm{K}_{*}(X)$ is the same as the reduced K-homology group of the one-point compactification of $X$.

Remark 1.4.19. The group $\mathrm{K}_{*}^{\Gamma}\left(X \times \mathbb{R}_{\geqslant 0}\right)$ always vanishes if $\Gamma$ acts freely and properly on $X$ and trivially on $\mathbb{R}_{\geqslant 0}$. This follows because the one-point compactification of $(\Gamma \backslash X) \times \mathbb{R}_{\geqslant 0}$ is contractible; it is simply the cone over the one-point compactification of $\Gamma \backslash X$. Alternatively it can be proved directly in the analytic setup via an Eilenberg swindle (see also Subsection 2.1.3 for a similar statement in the coarse category).

Moreover, as previously mentioned, Yu's localization algebras provide an alternative approach to the K-homology of proper metric spaces: 
Theorem 1.4.20 ([Yu97; QR10]). Let $X$ be a proper metric space endowed with a proper and free isometric action by a discrete group $\Gamma$. Then there exists an isomorphism $\operatorname{Ind}_{\mathrm{L}}^{\Gamma}: \mathrm{K}_{*}^{\Gamma}(X) \stackrel{\cong}{\rightarrow} \mathrm{K}_{*}\left(\mathrm{C}_{\Gamma}^{*} \mathrm{~L}(X)\right)$ such that the equivariant coarse index map is described by the following commutative diagram:

$$
\mathrm{K}_{*}^{\Gamma}(X) \stackrel{\operatorname{Ind}_{\mathrm{L}}^{\Gamma}}{\longrightarrow} \mathrm{K}_{*}\left(\mathrm{C}_{\Gamma}^{*} \mathrm{~L}(X)\right)
$$

In further chapters of this thesis, we will use Theorem 1.4.20 implicitly by suppressing the isomorphism $\operatorname{Ind}_{\mathrm{L}}^{\Gamma}$ and simply identifying $\mathrm{K}_{*}^{\Gamma}(X)$ with $\mathrm{K}_{*}\left(\mathrm{C}_{\Gamma}^{*} \mathrm{~L}(X)\right)$ whenever it is appropriate and convenient. 



\section{Secondary large-scale index theory for psc}

This chapter grew out of the author's article [Zei16] and constitutes the core part of this thesis. Here we develop our theory of secondary invariants for Riemannian metrics of positive scalar curvature outside a given subset and study the receptacles of these invariants.

\subsection{Partial structure groups}

In this section, we introduce and study the partial structure groups from an abstract point of view. These groups will serve as the receptacles for secondary invariants associated to metrics of uniformly positive scalar curvature outside a given subset.

Definition 2.1.1. Let $X$ be a proper metric space endowed with a free and proper $\Gamma$-action by isometries. Let $A \subseteq X$ be a $\Gamma$-invariant subset. The partial structure group of $X$ relative to $A$ is defined as

$$
\mathrm{S}_{*}^{\Gamma}(X / / A):=\mathrm{K}_{*}\left(\mathrm{C}_{\Gamma}^{*} \mathrm{~L}_{A}(X)\right)
$$

In the special case of $A=\emptyset$, we write $\mathrm{S}_{*}^{\Gamma}(X):=\mathrm{S}_{*}^{\Gamma}(X / / \emptyset)=\mathrm{K}_{*}\left(\mathrm{C}_{\Gamma}^{*} \mathrm{~L}_{0}(X)\right)$; this agrees with the analytic structure group of Higson-Roe (compare [XY14b, Section 6]).

Moreover, as indicated in Subsection 1.4.3 and justified by Theorem 1.4.20, we identify

$$
\mathrm{K}_{*}^{\Gamma}(X)=\mathrm{S}_{*}^{\Gamma}(X / / X)=\mathrm{K}_{*}\left(\mathrm{C}_{\Gamma}^{*} \mathrm{~L}(X)\right) .
$$

Remark 2.1.2. Remark 1.4.17 implies that for all $n \in \mathbb{N}$ there is a canonical identification

$$
\mathrm{S}_{n}^{\Gamma}(X / / A)=\mathrm{K}_{0}\left(\mathrm{C}_{\Gamma}^{*} \mathrm{~L}_{A}\left(X ; \mathrm{Cl}_{n}\right)\right)
$$

We will use this heavily in our construction of secondary invariants.

\subsubsection{Generalized Higson-Roe sequences}

A main feature of the analytic structure group is that it fits into a long exact sequence with the coarse index map (see for instance [HR05]), the so-called Higson-Roe sequence. The following long exact sequences involving the partial structure groups are straightforward generalizations of the Higson-Roe sequence: 
Proposition 2.1.3. In the setup of Definition 2.1.1, let $A \subseteq B \subseteq X$ be $\Gamma$-invariant subsets. Then there is a long exact sequence:

$$
\begin{gathered}
\mathrm{S}_{*+1}^{\Gamma}(X / / A) \stackrel{q_{A, B}^{\Gamma}}{\longrightarrow} \mathrm{S}_{*+1}^{\Gamma}(X / / B) \stackrel{\operatorname{Ind}_{B / / A}^{\Gamma}}{\longrightarrow} \mathrm{K}_{*+1}\left(\frac{\mathrm{C}_{\Gamma}^{*}(B)}{\mathrm{C}_{\Gamma}^{*}(A \subset B)}\right) \\
\mathrm{S}_{*}^{\Gamma}(X / / A) \stackrel{q_{A, B}^{\Gamma}}{\longrightarrow} S_{*}^{\Gamma}(X / / B) \stackrel{\operatorname{Ind}_{B / / A}^{\Gamma}}{\longrightarrow} \mathrm{K}_{*}\left(\frac{\mathrm{C}_{\Gamma}^{*}(B)}{\mathrm{C}_{\Gamma}^{*}(A \subset B)}\right)
\end{gathered}
$$

Proof. The sequence is the long exact sequence in K-theory associated to the following short exact sequence:

$$
0 \rightarrow \mathrm{C}_{\Gamma}^{*} \mathrm{~L}_{A}(X) \rightarrow \mathrm{C}_{\Gamma}^{*} \mathrm{~L}_{B}(X) \stackrel{\pi \text { oev }_{1}}{\longrightarrow} \frac{\mathrm{C}_{\Gamma}^{*}(B \subset X)}{\mathrm{C}_{\Gamma}^{*}(A \subset X)} \rightarrow 0,
$$

where $\pi: \mathrm{C}_{\Gamma}^{*}(B \subset X) \rightarrow \frac{\mathrm{C}_{\Gamma}^{*}(B \subset X)}{\mathrm{C}_{\Gamma}^{*}(A \subset X)}$ is the canonical quotient map. We also use Lemma 1.4.18.

We explicitly note the following important special cases:

Corollary 2.1.4. In the situation of Definition 2.1.1, there are long exact sequences

$$
\begin{gathered}
\mathrm{S}_{*+1}^{\Gamma}(X) \stackrel{q_{0, A}^{\Gamma}}{\longrightarrow} \mathrm{S}_{*+1}^{\Gamma}(X / / A) \stackrel{\operatorname{Ind}_{A}^{\Gamma}}{\longrightarrow} \mathrm{K}_{*+1}\left(\mathrm{C}_{\Gamma}^{*}(A)\right) \\
\mathrm{S}_{*}^{\Gamma}(X) \stackrel{q_{0, A}^{\Gamma}}{\longrightarrow} \mathrm{S}_{*}^{\Gamma}(X / / A) \stackrel{\partial_{A}^{\Gamma}}{\stackrel{\operatorname{Ind}_{A}^{\Gamma}}{\longrightarrow}} \mathrm{K}_{*}\left(\mathrm{C}_{\Gamma}^{*}(A)\right),
\end{gathered}
$$

and

$$
\begin{aligned}
& \mathrm{S}_{*+1}^{\Gamma}(X / / A) \stackrel{q_{A, X}^{\Gamma}}{\longrightarrow} \mathrm{K}_{*+1}^{\Gamma}(X) \stackrel{\operatorname{Ind}_{/ / A}^{\Gamma}}{\longrightarrow} \mathrm{K}_{*+1}\left(\frac{\mathrm{C}_{\Gamma}^{*}(X)}{\mathrm{C}_{\Gamma}^{*}(A \subset X)}\right)
\end{aligned}
$$

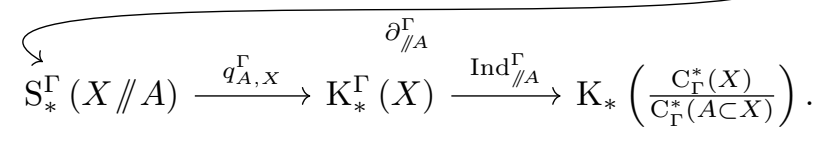

\subsubsection{Mayer-Vietoris sequences}

Let $X=X_{1} \cup X_{2}$ be a cover of a proper metric space by two closed subspaces. We wish to construct Mayer-Vietoris sequences relating the K-theory groups of the localization algebras (and hence of the partial structure groups) of the spaces $X, X_{1}, X_{2}$ and $X_{1} \cap X_{2}$. To do this, the general principle is to employ the ideals associated to subspaces from Definition 1.4.7 and then apply the abstract Mayer-Vietoris sequence 
we have discussed in Subsection 1.1.4. For the Roe algebra this has been implemented in [HRY93]. A recent treatment, which also deals with the structure algebra, can be found in [Sie12b]. In this section, we sketch how to carry out this program for localization algebras, compare [Yu97].

Definition 2.1.5. A cover $X=X_{1} \cup X_{2}$ by two subsets $X_{1}, X_{2}$ is coarsely excisive if there exists a function $S: \mathbb{R}_{>0} \rightarrow \mathbb{R}_{>0}$ such that

$$
\mathcal{U}_{R}\left(X_{1}\right) \cap \mathcal{U}_{R}\left(X_{2}\right) \subseteq \mathcal{U}_{S(R)}\left(X_{1} \cap X_{2}\right) \text { for all } R>0
$$

If, in addition, the function $S$ can be chosen such that $S(t) \rightarrow 0$ as $t \rightarrow 0$, then we say that the cover is uniformly excisive.

If the cover $X=X_{1} \cup X_{2}$ is coarsely excisive with $X_{1}, X_{2}$ closed and $X_{1} \cap X_{2} \Gamma$ cocompact, then a compactness argument shows that it is uniformly excisive. Moreover, if $X$ is a geodesic metric space, then any cover by two closed subsets is automatically uniformly excisive.

The following notation will be used in the proofs below: For a subset $A \subseteq X$, we denote by $\mathbf{1}_{A}$ the projection on an $X$-module corresponding to the characteristic function of $A$.

Lemma 2.1.6 (compare [HRY93]). Let $X=X_{1} \cup X_{2}$ be a cover and let $A \subseteq X$ be a subset such that the cover of $A$ by $A \cap X_{1}$ and $A \cap X_{2}$ is coarsely excisive. Then:

$$
\begin{aligned}
& \mathrm{C}_{\Gamma}^{*}\left(A \cap X_{1} \subset X\right) \cap \mathrm{C}_{\Gamma}^{*}\left(A \cap X_{2} \subset X\right)=\mathrm{C}_{\Gamma}^{*}\left(A \cap X_{1} \cap X_{2} \subset X\right), \\
& \mathrm{C}_{\Gamma}^{*}\left(A \cap X_{1} \subset X\right)+\mathrm{C}_{\Gamma}^{*}\left(A \cap X_{2} \subset X\right)=\mathrm{C}_{\Gamma}^{*}(A \subset X) .
\end{aligned}
$$

Proof. To show the first equality, we proceed as in Remark 1.4.13. Since the cover is coarsely excisive, we observe

$$
\mathbf{C}\left[A \cap X_{1} \subset X\right]^{\Gamma} \cap \mathbf{C}\left[A \cap X_{2} \subset X\right]^{\Gamma} \subseteq \mathbf{C}\left[A \cap X_{1} \cap X_{2} \subset X\right]^{\Gamma} .
$$

We conclude that

$$
\begin{aligned}
\mathrm{C}_{\Gamma}^{*}\left(A \cap X_{1} \subset X\right) \cap \mathrm{C}_{\Gamma}^{*}\left(A \cap X_{2} \subset X\right) & =\mathrm{C}_{\Gamma}^{*}\left(A \cap X_{1} \subset X\right) \mathrm{C}_{\Gamma}^{*}\left(A \cap X_{2} \subset X\right) \\
& =\overline{\mathbf{C}\left[A \cap X_{1} \subset X\right]^{\Gamma} \mathbf{C}\left[A \cap X_{2} \subset X\right]^{\Gamma}} \\
& \subseteq \overline{\mathbf{C}\left[A \cap X_{1} \cap X_{2} \subset X\right]^{\Gamma}} \\
& =\mathrm{C}_{\Gamma}^{*}\left(A \cap X_{1} \cap X_{2} \subset X\right) .
\end{aligned}
$$

The converse inclusion of the first equality is straightforward.

To show the second equality, it suffices to prove that

$$
\mathbf{C}[A \subset X]^{\Gamma} \subseteq \mathbf{C}\left[A \cap X_{1} \subset X\right]^{\Gamma}+\mathbf{C}\left[A \cap X_{2} \subset X\right]^{\Gamma} .
$$

Indeed, let $T \in \mathbf{C}[A \subset X]^{\Gamma}$ and choose $R>0$ such that $\operatorname{prop}(T)<R$ and $\operatorname{supp}(T) \subseteq$ $\mathcal{U}_{R}(A) \times \mathcal{U}_{R}(A)$. Now set $T_{1}:=\mathbf{1}_{\mathcal{U}_{R}\left(A \cap X_{1}\right)} T$ and $T_{2}:=T-T_{1}$. Then

$$
\operatorname{supp}\left(T_{1}\right) \subseteq \operatorname{supp}(T) \cap\left(\mathcal{U}_{R}\left(A \cap X_{1}\right) \times X\right) \subseteq \mathcal{U}_{R}\left(A \cap X_{1}\right) \times \mathcal{U}_{2 R}\left(A \cap X_{1}\right),
$$


where the second inclusion follows from $\operatorname{prop}(T)<R$. Hence $T_{1} \in \mathbf{C}\left[A \cap X_{1} \subset X\right]^{\Gamma}$. Moreover, $T_{2} \in \mathbf{C}\left[A \cap X_{2} \subset X\right]^{\Gamma}$ as the following calculation shows:

$$
\begin{aligned}
\operatorname{supp}\left(T_{2}\right) & \subseteq \operatorname{supp}(T) \cap\left(X \backslash \mathcal{U}_{R}\left(A \cap X_{1}\right) \times X\right) \\
& \subseteq \operatorname{supp}(T) \cap\left(\mathcal{U}_{R}(A) \times \mathcal{U}_{R}(A)\right) \cap\left(X \backslash \mathcal{U}_{R}\left(A \cap X_{1}\right) \times X\right) \\
& \subseteq \operatorname{supp}(T) \cap\left(\mathcal{U}_{R}\left(A \cap X_{2}\right) \times \mathcal{U}_{R}(A)\right) \\
& \subseteq\left(\mathcal{U}_{R}\left(A \cap X_{2}\right) \times \mathcal{U}_{2 R}\left(A \cap X_{2}\right)\right) .
\end{aligned}
$$

Hence $T=T_{1}+T_{2} \in \mathbf{C}\left[A \cap X_{1} \subset X\right]^{\Gamma}+\mathbf{C}\left[A \cap X_{2} \subset X\right]^{\Gamma}$.

Lemma 2.1.7. Let $X=X_{1} \cup X_{2}$ be a uniformly excisive cover and $A \subseteq X$ a subset such that the cover of $A$ by $A \cap X_{1}$ and $A \cap X_{2}$ is coarsely exicive. Then

$$
\begin{aligned}
& \mathrm{C}_{\Gamma}^{*} \mathrm{~L}_{A \cap X_{1}}\left(X_{1} \subset X\right) \cap \mathrm{C}_{\Gamma}^{*} \mathrm{~L}_{A \cap X_{2}}\left(X_{2} \subset X\right)=\mathrm{C}_{\Gamma}^{*} \mathrm{~L}_{A \cap X_{1} \cap X_{2}}\left(X_{1} \cap X_{2} \subset X\right), \\
& \mathrm{C}_{\Gamma}^{*} \mathrm{~L}_{A \cap X_{1}}\left(X_{1} \subset X\right)+\mathrm{C}_{\Gamma}^{*} \mathrm{~L}_{A \cap X_{2}}\left(X_{2} \subset X\right)=\mathrm{C}_{\Gamma}^{*} \mathrm{~L}_{A}(X) .
\end{aligned}
$$

Proof. The proof of the first equality can be carried out as in Lemma 2.1.6. To show the second equality, we need to extend the idea somewhat.

Since the sum of two closed ideals in a $\mathrm{C}^{*}$-algebra is always closed, it suffices to show that $\mathbf{C L}_{A \cap X_{1}}\left[X_{1} \subset X\right]^{\Gamma}+\mathbf{C L}_{A \cap X_{2}}\left[X_{2} \subset X\right]^{\Gamma}$ is dense in $\mathbf{C L}_{A}[X]^{\Gamma}$ (and hence in $\left.\mathrm{C}_{\Gamma}^{*} \mathrm{~L}_{A}(X)\right)$. So let $L \in \mathbf{C L}_{A}[X]^{\Gamma}$ and set $T:=L(1)$. We may assume that there exists an $1>\varepsilon>0$ such that $L(t)=T$ for all $1 \leqslant t<1+\varepsilon$; this is because the set of all such $L$ is dense in $\mathbf{C L}[X]^{\Gamma}$ by continuity and a linear interpolation argument. Choose $R>0$ such that $\operatorname{prop}(T)<R$ and $\operatorname{supp}(T) \subseteq \mathcal{U}_{R}(A) \times \mathcal{U}_{R}(A)$. Let $L_{1}(t):=P(t) L(t)$, where

$$
P(t):= \begin{cases}\frac{1}{\varepsilon}\left((1+\varepsilon-t) \mathbf{1}_{\mathcal{U}_{R}\left(X_{1} \cap A\right)}+(t-1) \mathbf{1}_{\mathcal{U}_{R}\left(X_{1}\right)}\right) & t \in[1,1+\varepsilon], \\ \frac{1}{1-\varepsilon}\left((2-t) \mathbf{1}_{\mathcal{U}_{R}\left(X_{1}\right)}+(t-1-\varepsilon) \mathbf{1}_{X_{1}}\right) & t \in[1+\varepsilon, 2], \\ \mathbf{1}_{X_{1}} & t \in[2, \infty) .\end{cases}
$$

Moreover, we set $L_{2}:=L-L_{1}$. Then $T_{1}:=L_{1}(1)=\mathbf{1}_{\mathcal{U}_{R}\left(X_{1} \cap A\right)} T$ and $T_{2}:=$ $L_{2}(1)=\mathbf{1}_{X \backslash \mathcal{U}_{R}\left(X_{1} \cap A\right)} T$. Hence it follows from the proof of Lemma 2.1.6 that $T_{1} \in \mathbf{C}\left[A \cap X_{1} \subset X\right]^{\Gamma}$ and $T_{2} \in \mathbf{C}\left[A \cap X_{2} \subset X\right]^{\Gamma}$.

It follows from the definition of $L_{1}$ that

$$
\begin{aligned}
& \operatorname{supp}\left(L_{1}(t)\right) \subseteq \mathcal{U}_{R}\left(X_{1}\right) \times \mathcal{U}_{R+\operatorname{prop}(L(t))}\left(X_{1}\right) \quad t \in[1,2] \\
& \operatorname{supp}\left(L_{1}(t)\right) \subseteq X_{1} \times \mathcal{U}_{\operatorname{prop}(L(t))}\left(X_{1}\right) \quad t \in[2, \infty) .
\end{aligned}
$$

Since $\operatorname{prop}(L(t)) \rightarrow 0$ as $t \rightarrow \infty$, we deduce that $L_{1} \in \mathbf{C L}_{A \cap X_{1}}\left[X_{1} \subset X\right]^{\Gamma}$.

Furthermore, for $t \in[1,1+\varepsilon]$, the support of $1-P(t)$ is contained in $X \backslash \mathcal{U}_{R}\left(A \cap X_{1}\right)$. Therefore $\operatorname{supp}\left(L_{2}(t)\right) \subseteq \operatorname{supp}(T) \cap\left(X \backslash \mathcal{U}_{R}\left(X_{1} \cap A\right) \times X\right)$ for $t \in[1,1+\varepsilon]$ and the same argument as for $T_{2}$ in Lemma 2.1.6 shows that

$$
L_{2}(t) \in \mathbf{C}\left[X_{2} \cap A \subset X\right]^{\Gamma} \quad \text { for } t \in[1,1+\varepsilon] .
$$


Finally, since the support of $1-P(t)$ is contained in $X_{2}$ for $t \in[1+\varepsilon, \infty)$, an analogous argument as for $L_{1}$ shows that

$$
\operatorname{supp}\left(L_{2}(t)\right) \subseteq X_{2} \times \mathcal{U}_{\operatorname{prop}(L(t))}\left(X_{2}\right) \text { for } t \in[1+\varepsilon, \infty)
$$

and hence $L_{2} \in \mathbf{C L}_{A \cap X_{2}}\left[X_{2} \subset X\right]^{\Gamma}$.

In view of Lemmas 2.1.6, 2.1.7 and 1.4.18, the abstract Mayer-Vietoris sequence from Subsection 1.1.4 yields (compatible) Mayer-Vietoris sequences for the K-theory of the Roe algebra and the partial structure groups. We summarize this in the following propositions:

Proposition 2.1.8 ([HRY93]). Let $X=X_{1} \cup X_{2}$ be a cover and let $A \subset X$ be a $\Gamma$-invariant subset. Suppose that $A=\left(A \cap X_{1}\right) \cup\left(A \cap X_{2}\right)$ is a coarsely excisive cover. Then there is a long exact Mayer-Vietoris sequence as follows:

$$
\begin{array}{r}
\mathrm{K}_{*+1}\left(\mathrm{C}_{\Gamma}^{*}\left(A \cap X_{1} \cap X_{2}\right)\right) \longrightarrow \begin{array}{l}
\mathrm{K}_{*+1}\left(\mathrm{C}_{\Gamma}^{*}\left(A \cap X_{1}\right)\right) \\
\mathrm{K}_{*+1}\left(\mathrm{C}_{\Gamma}^{*}\left(A \cap X_{2}\right)\right)
\end{array} \longrightarrow \mathrm{K}_{*+1}\left(\mathrm{C}_{\Gamma}^{*}(A)\right) \\
\mathrm{K}_{*}\left(\mathrm{C}_{\Gamma}^{*}\left(A \cap X_{1} \cap X_{2}\right)\right) \longrightarrow \mathrm{K}_{*}\left(\mathrm{C}_{\Gamma}^{*}(A)\right) . \\
\begin{array}{l}
\mathrm{K}_{*}\left(\mathrm{C}_{\Gamma}^{*}\left(A \cap X_{1}\right)\right) \\
\mathrm{K}_{*}\left(\mathrm{C}_{\Gamma}^{*}\left(A \cap X_{2}\right)\right)
\end{array}
\end{array}
$$

Proposition 2.1.9 (compare [Sie12b] for $A=\emptyset$ ). Let $X=X_{1} \cup X_{2}$ be uniformly excisive and let $A \subset X$ be a closed $\Gamma$-invariant subset. Suppose furthermore that $A=\left(A \cap X_{1}\right) \cup\left(A \cap X_{2}\right)$ is a coarsely excisive cover. Then there is a long exact Mayer-Vietoris sequence as follows:

$$
\begin{aligned}
& \mathrm{S}_{*+1}^{\Gamma}\left(X_{1} \cap X_{2} / / A \cap X_{1} \cap X_{2}\right) \longrightarrow \underset{\mathrm{S}_{*+1}^{\Gamma}\left(X_{2} / / A \cap X_{2}\right)}{\mathrm{S}_{*+1}^{\Gamma}\left(X_{1} / / A \cap X_{1}\right)} \rightarrow \mathrm{S}_{*+1}^{\Gamma}(X / / A) \\
& \mathrm{S}_{*}^{\Gamma}\left(X_{1} \cap X_{2} / / A \cap X_{1} \cap X_{2}\right) \longrightarrow \mathrm{S}_{*}^{\Gamma}(X / / A) .
\end{aligned}
$$

Moreover, the index maps $\operatorname{Ind}_{A \cap Y}^{\Gamma}: \mathrm{S}_{*}^{\Gamma}(Y / / A \cap Y) \rightarrow \mathrm{K}_{*}\left(\mathrm{C}_{\Gamma}^{*}(A \cap Y)\right)$ where $Y$ ranges over $\left\{X, X_{1}, X_{2}, X_{1} \cap X_{2}\right\}$ intertwine this sequence with the sequence from Proposition 2.1.8.

Under an additional condition, there is also a Mayer-Vietoris sequence for the $\mathrm{K}$ theory of the quotient algebras $\mathrm{C}_{\Gamma}^{*}(X) / \mathrm{C}_{\Gamma}^{*}(A \subset X)$. We start with a technical lemma before stating the result. 
Lemma 2.1.10. Let $X=X_{1} \cup X_{2}$ be a coarsely excicive cover and let $A \subset X$ be a subset such that

$$
\forall R>0 \exists S>0: \quad \mathcal{U}_{R}\left(A \cap X_{2}\right) \cap \mathcal{U}_{R}\left(X_{1} \cap X_{2}\right) \subseteq \mathcal{U}_{S}\left(A \cap X_{1} \cap X_{2}\right) .
$$

Then the cover of $A$ by $X_{1} \cap A$ and $X_{2} \cap A$ is coarsely excicive and

$$
\forall R>0 \exists S>0: \quad \mathcal{U}_{R}(A) \cap \mathcal{U}_{R}\left(X_{1}\right) \subseteq \mathcal{U}_{S}\left(A \cap X_{1}\right) .
$$

Proof. Let $R>0$. Choose $\tilde{S} \geqslant R$ such that $\mathcal{U}_{R}\left(X_{1}\right) \cap \mathcal{U}_{R}\left(X_{2}\right) \subseteq \mathcal{U}_{\tilde{S}}\left(X_{1} \cap X_{2}\right)$. By (2.1.4), there exists $S \geqslant \tilde{S}$ such that $\mathcal{U}_{\tilde{S}}\left(A \cap X_{2}\right) \cap \mathcal{U}_{\tilde{S}}\left(X_{1} \cap X_{2}\right) \subseteq \mathcal{U}_{S}\left(A \cap X_{1} \cap X_{2}\right)$. We deduce

$$
\begin{aligned}
\mathcal{U}_{R}(A) \cap \mathcal{U}_{R}\left(X_{1}\right) & \subseteq\left(\mathcal{U}_{R}\left(A \cap X_{1}\right) \cup \mathcal{U}_{R}\left(A \cap X_{2}\right)\right) \cap \mathcal{U}_{R}\left(X_{1}\right) \\
& \subseteq \mathcal{U}_{R}\left(A \cap X_{1}\right) \cup\left(\mathcal{U}_{R}\left(A \cap X_{2}\right) \cap \mathcal{U}_{R}\left(X_{1}\right)\right) \\
& \subseteq \mathcal{U}_{R}\left(A \cap X_{1}\right) \cup\left(\mathcal{U}_{R}\left(A \cap X_{2}\right) \cap \mathcal{U}_{R}\left(X_{2}\right) \cap \mathcal{U}_{R}\left(X_{1}\right)\right) \\
& \subseteq \mathcal{U}_{R}\left(A \cap X_{1}\right) \cup\left(\mathcal{U}_{\tilde{S}}\left(A \cap X_{2}\right) \cap \mathcal{U}_{\tilde{S}}\left(X_{1} \cap X_{2}\right)\right) \\
& \subseteq \mathcal{U}_{R}\left(A \cap X_{1}\right) \cup \mathcal{U}_{S}\left(A \cap X_{1} \cap X_{2}\right) \\
& \subseteq \mathcal{U}_{S}\left(A \cap X_{1}\right) .
\end{aligned}
$$

Similarly,

$$
\begin{aligned}
\mathcal{U}_{R}\left(A \cap X_{1}\right) \cap \mathcal{U}_{R}\left(A \cap X_{2}\right) & \subseteq \mathcal{U}_{R}\left(X_{1}\right) \cap \mathcal{U}_{R}\left(X_{2}\right) \cap \mathcal{U}_{R}\left(A \cap X_{2}\right) \\
& \subseteq \mathcal{U}_{\tilde{S}}\left(X_{1} \cap X_{2}\right) \cap \mathcal{U}_{\tilde{S}}\left(A \cap X_{2}\right) \\
& \subseteq \mathcal{U}_{S}\left(A \cap X_{1} \cap X_{2}\right) .
\end{aligned}
$$

Proposition 2.1.11. Let $X=X_{1} \cup X_{2}$ be a coarsely excicive cover and let $A \subset X$ be a $\Gamma$-invariant subset such that

$$
\forall R>0 \exists S>0: \quad \mathcal{U}_{R}(A) \cap \mathcal{U}_{R}\left(X_{1} \cap X_{2}\right) \subseteq \mathcal{U}_{S}\left(A \cap X_{1} \cap X_{2}\right) .
$$

Then there is a long exact Mayer-Vietoris sequence as follows:

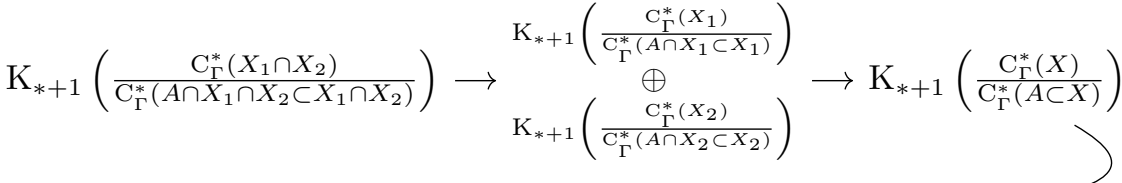

$$
\begin{aligned}
& \mathrm{K}_{*}\left(\frac{\mathrm{C}_{\Gamma}^{*}\left(X_{1} \cap X_{2}\right)}{\mathrm{C}_{\Gamma}^{*}\left(A \cap X_{1} \cap X_{2} \subset X_{1} \cap X_{2}\right)}\right) \longrightarrow \mathrm{K}_{*}\left(\frac{\mathrm{C}_{\Gamma}^{*}(X)}{\mathrm{C}_{\Gamma}^{*}(A \subset X)}\right) .
\end{aligned}
$$


Proof. Condition (2.1.6) implies both (2.1.4) itself and (2.1.4) with the roles of $X_{1}$ and $X_{2}$ swapped. Thus the cover of $A$ by $A \cap X_{1}$ and $A \cap X_{2}$ is also coarsely excicive and for $i \in\{1,2\}$

$$
\forall R>0 \exists S>0: \quad \mathcal{U}_{R}(A) \cap \mathcal{U}_{R}\left(X_{i}\right) \subseteq \mathcal{U}_{S}\left(A \cap X_{i}\right) .
$$

Thus Lemma 2.1.6 implies:

$$
\begin{gathered}
\mathrm{C}_{\Gamma}^{*}(A \subset X) \cap \mathrm{C}_{\Gamma}^{*}\left(X_{1} \cap X_{2} \subset X\right)=\mathrm{C}_{\Gamma}^{*}\left(A \cap X_{1} \cap X_{2} \subset X\right), \\
\mathrm{C}_{\Gamma}^{*}(A \subset X) \cap \mathrm{C}_{\Gamma}^{*}\left(X_{i} \subset X\right)=\mathrm{C}_{\Gamma}^{*}\left(A \cap X_{i} \subset X\right),
\end{gathered}
$$

Hence there are injections for $i \in\{1,2\}$ as follows

$$
\frac{\mathrm{C}_{\Gamma}^{*}\left(X_{1} \cap X_{2} \subset X\right)}{\mathrm{C}_{\Gamma}^{*}\left(A \cap X_{1} \cap X_{2} \subset X\right)} \hookrightarrow \frac{\mathrm{C}_{\Gamma}^{*}\left(X_{i} \subset X\right)}{\mathrm{C}_{\Gamma}^{*}\left(A \cap X_{i} \subset X\right)} \hookrightarrow \frac{\mathrm{C}_{\Gamma}^{*}(X)}{\mathrm{C}_{\Gamma}^{*}(A \subset X)} .
$$

Now Lemma 2.1.6 implies

$$
\begin{gathered}
\frac{\mathrm{C}_{\Gamma}^{*}\left(X_{1} \subset X\right)}{\mathrm{C}_{\Gamma}^{*}\left(A \cap X_{1} \subset X\right)}+\frac{\mathrm{C}_{\Gamma}^{*}\left(X_{2} \subset X\right)}{\mathrm{C}_{\Gamma}^{*}\left(A \cap X_{2} \subset X\right)}=\frac{\mathrm{C}_{\Gamma}^{*}(X)}{\mathrm{C}_{\Gamma}^{*}(A \subset X)}, \\
\frac{\mathrm{C}_{\Gamma}^{*}\left(X_{1} \subset X\right)}{\mathrm{C}_{\Gamma}^{*}\left(A \cap X_{1} \subset X\right)} \cap \frac{\mathrm{C}_{\Gamma}^{*}\left(X_{1} \subset X\right)}{\mathrm{C}_{\Gamma}^{*}\left(A \cap X_{1} \subset X\right)} \\
=\left(\frac{\mathrm{C}_{\Gamma}^{*}\left(X_{1} \subset X\right)}{\mathrm{C}_{\Gamma}^{*}\left(A \cap X_{1} \subset X\right)}\right)\left(\frac{\mathrm{C}_{\Gamma}^{*}\left(X_{1} \subset X\right)}{\mathrm{C}_{\Gamma}^{*}\left(A \cap X_{1} \subset X\right)}\right)=\frac{\mathrm{C}_{\Gamma}^{*}\left(X_{1} \cap X_{2} \subset X\right)}{\mathrm{C}_{\Gamma}^{*}\left(A \cap X_{1} \cap X_{2} \subset X\right)} .
\end{gathered}
$$

The existence of the stated Mayer-Vietoris sequence follows again from the abstract Mayer-Vietoris sequence from Subsection 1.1.4 and Lemma 1.4.18.

\subsubsection{Flasque spaces and suspension isomorphisms}

Definition 2.1.12. A proper metric space $X$ endowed with a free and proper $\Gamma$-action is called ( $\Gamma$-equivariantly) flasque if there exists a $\Gamma$-equivariant coarse map $s: X \rightarrow X$ such that

(i) $s$ is coarsely equivalent to $\operatorname{id}_{X}$,

(ii) for every compact subset $K \subseteq X$, there exists $l_{0} \in \mathbb{N}$ such that $s^{l}(X) \cap K=\emptyset$ for all $l \geqslant l_{0}$,

(iii) for all $R>0$, there exists $S>0$ such that $d_{X}\left(s^{l}(x), s^{l}\left(x^{\prime}\right)\right)<S$ for all $l \geqslant 0$ and $x, x^{\prime} \in X$ with $d_{X}\left(x, x^{\prime}\right)<R$.

By definition, $X \times Y$ is flasque if $X$ is flasque and $Y$ is an arbitrary proper metric space.

An Eilenberg swindle argument implies the following:

Proposition 2.1.13 (see [Roe96, Proposition 9.4], [HR00, Lemma 6.4.2]). If $X$ is $\Gamma$-equivariantly flasque, then $\mathrm{K}_{*}\left(\mathrm{C}_{\Gamma}^{*}(X)\right)=0$ in all degrees. 
Corollary 2.1.14. Let $X$ be a proper metric space endowed with a free and proper $\Gamma$-action. Then the $\mathrm{K}$-theory groups $\mathrm{K}_{*}\left(\mathrm{C}_{\Gamma}^{*}\left(X \times \mathbb{R}_{\geqslant 0}\right)\right)$ and $\mathrm{K}_{*}\left(\mathrm{C}_{\Gamma}^{*}\left(X \times \mathbb{R}_{\leqslant 0}\right)\right)$ vanish in all degrees. In particular, the Mayer-Vietoris boundary map

$$
\partial_{\mathrm{MV}}: \mathrm{K}_{*+1}\left(\mathrm{C}_{\Gamma}^{*}(X \times \mathbb{R})\right) \rightarrow \mathrm{K}_{*}\left(\mathrm{C}_{\Gamma}^{*}(X)\right)
$$

associated to the cover $X \times \mathbb{R}=X \times \mathbb{R}_{\geqslant 0} \cup X \times \mathbb{R}_{\leqslant 0}$ is an isomorphism.

It is also possible to define suitable notions of flasqueness that imply vanishing of the K-homology and (partial) structure groups. The following will suffice for our purposes:

Proposition 2.1.15. Let $X$ be a proper metric space endowed with a free and proper $\Gamma$-action and $A \subseteq X$ some $\Gamma$-invariant subset. Then $\mathrm{S}_{*}^{\Gamma}\left(X \times \mathbb{R}_{\geqslant 0} / / A \times \mathbb{R}_{\geqslant 0}\right)$ and $\mathrm{S}_{*}^{\Gamma}\left(X \times \mathbb{R}_{\leqslant 0} / / A \times \mathbb{R}_{\leqslant 0}\right)$ vanish. In particular, the Mayer-Vietoris boundary map

$$
\partial_{\mathrm{MV}}: \mathrm{S}_{*+1}^{\Gamma}(X \times \mathbb{R} / / A \times \mathbb{R}) \rightarrow \mathrm{S}_{*}^{\Gamma}(X / / A)
$$

associated to the cover $X \times \mathbb{R}=X \times \mathbb{R}_{\geqslant 0} \cup X \times \mathbb{R}_{\leqslant 0}$ is an isomorphism.

Proof. The statement holds for $A=X$ because $\mathrm{K}_{*}^{\Gamma}\left(X \times \mathbb{R}_{\geqslant 0}\right)$ vanishes by an Eilenberg swindle (or see Remark 1.4.19 for a topological explanation). Together with Corollary 2.1.14 and the exact sequence

$$
\mathrm{K}_{*+1}\left(\mathrm{C}_{\Gamma}^{*}\left(X \times \mathbb{R}_{\geqslant 0}\right)\right) \rightarrow \mathrm{S}_{*}^{\Gamma}\left(X \times \mathbb{R}_{\geqslant 0}\right) \rightarrow \mathrm{K}_{*}^{\Gamma}\left(X \times \mathbb{R}_{\geqslant 0}\right),
$$

this implies that $S_{*}^{\Gamma}\left(X \times \mathbb{R}_{\geqslant 0}\right)$ vanishes (this is the statement for $\left.A=\emptyset\right)$. Using this and Corollary 2.1.14 again, we deduce the result for a general subset $A$ from the exact sequence

$$
\mathrm{S}_{*}^{\Gamma}\left(X \times \mathbb{R}_{\geqslant 0}\right) \rightarrow \mathrm{S}_{*}^{\Gamma}\left(X \times \mathbb{R}_{\geqslant 0} / / A \times \mathbb{R}_{\geqslant 0}\right) \rightarrow \mathrm{K}_{*}\left(\mathrm{C}_{\Gamma}^{*}\left(A \times \mathbb{R}_{\geqslant 0}\right)\right) .
$$

\subsubsection{External products}

Let $X_{i}$ be proper metric spaces endowed with proper and free $\Gamma_{i}$-actions for $i=1,2$. Suppose that $\mathfrak{H}_{i}$ is a $\Gamma_{i}$-equivariant $\mathrm{Cl}_{n_{i}}$-linear ample $X_{i}$-module for $i=1,2$. Then $\mathfrak{H}:=\mathfrak{H}_{1} \widehat{\otimes} \mathfrak{H}_{2}$ is an ample $\Gamma$-equivariant $\mathrm{Cl}_{n}$-linear $X$-module for $\Gamma:=\Gamma_{1} \times \Gamma_{2}$ and $n:=n_{1}+n_{2}$. Then there is a canonical $*$-homomorphism

$$
\begin{aligned}
& \mathrm{t}_{X_{1}, X_{2}}: \mathrm{C}_{\Gamma_{1}}^{*} \mathrm{~L}\left(X_{1} ; \mathrm{Cl}_{n_{1}}\right) \widehat{\otimes} \mathrm{C}_{\Gamma_{2}}^{*} \mathrm{~L}\left(X_{2} ; \mathrm{Cl}_{n_{2}}\right) \rightarrow \mathrm{C}_{\Gamma_{1} \times \Gamma_{2}}^{*} \mathrm{~L}\left(X_{1} \times X_{2} ; \mathrm{Cl}_{n}\right), \\
& \left.\mathrm{t}_{X_{1}, X_{2}}\left(L_{1} \widehat{\otimes} L_{2}\right)(t)=L_{1}(t) \widehat{\otimes} L_{2}(t), \quad L_{i} \in \mathrm{C}_{\Gamma_{i}}^{*} \mathrm{~L}\left(X_{i} ; \mathrm{Cl}_{n_{i}}\right)\right), t \in[1, \infty] .
\end{aligned}
$$

If $Z_{1} \subset X_{1}$ is a $\Gamma_{1}$-invariant subset, then $\mathrm{t}_{X_{1}, X_{2}}$ restricts to a map

$$
\mathrm{t}_{X_{1}, X_{2}}: \mathrm{C}_{\Gamma_{1}}^{*} \mathrm{~L}_{Z_{1}}\left(X_{1} ; \mathrm{Cl}_{n_{1}}\right) \widehat{\otimes} \mathrm{C}_{\Gamma_{2}}^{*} \mathrm{~L}\left(X_{2} ; \mathrm{Cl}_{n_{2}}\right) \rightarrow \mathrm{C}_{\Gamma_{1} \times \Gamma_{2}}^{*} \mathrm{~L}_{Z_{1} \times X_{2}}\left(X_{1} \times X_{2} ; \mathrm{Cl}_{n}\right) .
$$

Combining this with the external product in K-theory, we obtain the following external product for the partial structure group:

$$
\mathrm{S}_{n_{1}}^{\Gamma_{1}}\left(X_{1} / / Z_{1}\right) \otimes \mathrm{K}_{n_{2}}^{\Gamma_{2}}\left(X_{1}\right) \stackrel{\otimes}{\rightarrow} \mathrm{S}_{n_{1}+n_{2}}^{\Gamma_{1} \times \Gamma_{2}}\left(X_{1} \times X_{2} / / Z_{1} \times X_{2}\right),
$$


where for each $x \in \mathrm{K}_{0}\left(\mathrm{C}_{\Gamma_{1}}^{*} \mathrm{~L}_{Z_{1}}\left(X_{1} ; \mathrm{Cl}_{n_{1}}\right)\right)$ and $y \in \mathrm{K}_{0}\left(\mathrm{C}_{\Gamma_{2}}^{*} \mathrm{~L}\left(X_{2} ; \mathrm{Cl}_{n_{2}}\right)\right)$, we define

$$
x \otimes y:=\left(\mathrm{t}_{X_{1}, X_{2}}\right)_{*}(x \times y) .
$$

The external product is functorial, that is, $\left(f_{1} \times f_{2}\right)_{*}(x \otimes y)=\left(f_{1}\right)_{*}(x) \otimes\left(f_{2}\right)_{*}(y)$ for ucc maps $f_{i}: X_{i} \rightarrow Y_{i}, i=1,2$.

Restricting the construction to $t=1$ yields

$$
\mathrm{t}_{X_{1}, X_{2}}: \mathrm{C}_{\Gamma_{1}}^{*}\left(X_{1}\right) \otimes \mathrm{C}_{\Gamma_{2}}^{*}\left(X_{2}\right) \rightarrow \mathrm{C}_{\Gamma_{1} \times \Gamma_{2}}^{*}\left(X_{1} \times X_{2}\right)
$$

and we obtain the external product for Roe algebras,

$$
\mathrm{K}_{n_{1}}\left(\mathrm{C}_{\Gamma_{1}}^{*}\left(X_{1}\right)\right) \otimes \mathrm{K}_{n_{2}}\left(\mathrm{C}_{\Gamma_{2}}^{*}\left(X_{2}\right)\right) \stackrel{\otimes}{\rightarrow} \mathrm{K}_{n_{1}+n_{2}}\left(\mathrm{C}_{\Gamma_{1} \times \Gamma_{2}}^{*}\left(X_{1} \times X_{2}\right)\right) .
$$

Let $Z_{1} \subseteq X_{1}$ be a $\Gamma_{1}$-invariant subset. Then

$$
\begin{aligned}
\mathrm{t}_{X_{1}, X_{2}}\left(\mathrm{C}_{\Gamma_{1}}^{*}\left(Z_{1} \subset X_{1} ; \mathrm{Cl}_{n_{1}}\right) \widehat{\otimes} \mathrm{C}_{\Gamma_{2}}^{*}\left(X_{2} ; \mathrm{Cl}_{n_{2}}\right)\right) & \\
& \subseteq \mathrm{C}_{\Gamma_{1} \times \Gamma_{2}}^{*}\left(Z_{1} \times X_{2} \subset X_{1} \times X_{2} ; \mathrm{Cl}_{n}\right) .
\end{aligned}
$$

Thus we also get an external product as follows:

$$
\begin{aligned}
\mathrm{K}_{n_{1}}\left(\frac{\mathrm{C}_{\Gamma_{1}}^{*}\left(X_{1}\right)}{\left.\mathrm{C}_{\Gamma_{1}}^{*}\left(Z_{1} \subset X_{1}\right)\right)}\right) \otimes \mathrm{K}_{n_{2}}\left(\mathrm{C}_{\Gamma_{2}}^{*}\left(X_{2}\right)\right) \\
\quad \stackrel{\otimes}{\rightarrow} \mathrm{K}_{n_{1}+n_{2}}\left(\frac{\mathrm{C}_{\Gamma_{1} \times \Gamma_{2}}^{*}\left(X_{1} \times X_{2}\right)}{\mathrm{C}_{\Gamma_{1} \times \Gamma_{2}}^{*}\left(Z_{1} \times X_{2} \subset X_{1} \times X_{2}\right)}\right) .
\end{aligned}
$$

\subsection{Definition of secondary invariants}

In this section, we combine the $\mathrm{Cl}_{n}$-linear localization algebras with the picture of K-theory from Section 1.1 in order to construct the (equivariant) fundamental classes and partial secondary invariants on complete spin manifolds. Indeed, an element of $\mathrm{K}_{0}\left(\mathrm{C}^{*} \mathrm{~L}\left(X ; \mathrm{Cl}_{n}\right)\right)$ may be defined by a $*$-homomorphism $\mathcal{S} \rightarrow \mathrm{C}^{*} \mathrm{~L}\left(X ; \mathrm{Cl}_{n}\right)$. Our definition of the fundamental class is essentially the $*$-homomorphism given by the functional calculus of the $\mathrm{Cl}_{n}$-linear spinor Dirac operator, see (2.2.1) below. A slight modification of this idea allows to define the (partial) secondary invariants in almost the same fashion in case the underlying Riemannian metric has uniformly positive scalar curvature, see Subsection 2.2.2. In Subsection 2.2.3, we then discuss a coarse version of the Atiyah-Patodi-Singer index for manifolds with (partial) positive scalar curvature at the boundary.

We will use the following conventions and shorthands to simplify the statements of several results:

Conventions. Manifolds are without boundary unless explicitly stated otherwise.

A "complete spin manifold" $X$ is a spin manifold together with a fixed admissible proper distance function $d_{X}$ in the sense of Section 1.3. 
A "complete spin manifold endowed with a $\Gamma$-action" is a complete spin manifold together with a proper and free action of a discrete group $\Gamma$ which preserves the spin structure and the fixed admissible proper distance function.

Although we allow greater generality in our definition, the only cases of proper distance functions we have in mind come from complete Riemannian metrics or the construction of attaching a cylindrical end (compare Subsection 1.3.3).

\subsubsection{The fundamental class via localization algebras}

Let $X$ be a complete spin $n$-manifold endowed with a $\Gamma$-action. Fix $g \in \mathcal{R}(X)^{\Gamma}$. Let $\mathcal{C}_{0}(X)$ act on sections of the spinor bundle $\Phi_{g}$ by multiplication operators. This turns $\mathrm{L}^{2}\left(\mathscr{\Phi}_{g}\right)$ it into an $\left(X, \Gamma, \mathrm{Cl}_{n}\right)$-module (compare Subsection 1.2.2). Using a measurable trivialization of $\Phi_{g}$ one can show that $\mathrm{L}^{2}\left(\Phi_{g}\right)$ is isomorphic to $\mathrm{L}^{2}(X) \widehat{\otimes} \mathrm{Cl}_{n}$, hence it is ample. In the following, we consider the localization algebra to be formed with respect to the distance function $d_{X}$ on the ample $\left(X, \Gamma, \mathrm{Cl}_{n}\right)$-module $\mathrm{L}^{2}\left(X, \Phi_{g}\right)$.

For $f \in \mathcal{S}$ and $t \in[1, \infty)$, define

$$
\alpha_{g}(f)(t):=f\left(\frac{1}{t} \mathscr{D}_{g}\right) \in \mathbb{B}\left(\mathrm{L}^{2}\left(X, \Phi_{g}\right)\right) .
$$

By [HR00, Proposition 10.5.2], $f\left(\frac{1}{t} \mathfrak{P}_{g}\right)$ is locally compact for each $t$. Moreover, if the (distributional) Fourier transform of $f \in \mathcal{C}_{0}(\mathbb{R})$ satisfies $\operatorname{supp}(\hat{f}) \subseteq[-R, R]$ for some $R>0$, then it follows from the unit propagation speed property of Dirac wave operators that $\operatorname{prop}\left(f\left(\mathscr{D}_{g}\right)\right) \leqslant R$, see [HR00, Chapter 10.3]. In this case, if we set $f_{t}(x):=f\left(t^{-1} x\right)$, then $\hat{f}_{t}(s)=t \hat{f}(t s)$ and hence $\operatorname{supp}\left(\hat{f}_{t}\right) \subseteq\left[-\frac{R}{t}, \frac{R}{t}\right]$. This demonstrates that $\operatorname{prop}\left(f\left(\frac{1}{t} \mathscr{D}_{g}\right)\right) \rightarrow 0$ as $t \rightarrow \infty$ whenever $\hat{f}$ is compactly supported. We deduce that $\alpha_{g}(f) \in \mathbf{C L}\left[X ; \mathrm{Cl}_{n}\right]^{\Gamma}$ for all $f \in \mathcal{S}$ with compactly supported Fourier transform. Since those functions are dense in $\mathcal{S}$, we conclude that $\alpha_{g}(f) \in \mathrm{C}_{\Gamma}^{*} \mathrm{~L}\left(X ; \mathrm{Cl}_{n}\right)$ for all $f \in \mathcal{S}$. Thus $\alpha_{g}$ defines a $*$-homomorphism

$$
\alpha_{g}: \mathcal{S} \rightarrow \mathrm{C}_{\Gamma}^{*} \mathrm{~L}\left(X ; \mathrm{Cl}_{n}\right),
$$

which preserves the grading since $\mathscr{D}_{g}$ is an odd operator (use [HR00, Lemma 10.6.2]).

Definition 2.2.1. The equivariant fundamental class of $X$ is the K-theory class represented by the $*$-homomorphism $\alpha_{g}$ above,

$$
[X]^{\Gamma}=\left[\alpha_{g}\right] \in \mathrm{K}_{0}\left(\mathrm{C}_{\Gamma}^{*} \mathrm{~L}\left(X ; \mathrm{Cl}_{n}\right)\right) \cong \mathrm{K}_{n}\left(\mathrm{C}_{\Gamma}^{*} \mathrm{~L}(X)\right)=\mathrm{K}_{n}^{\Gamma}(X) .
$$

Definition 2.2.2. The (equivariant) coarse index of $X$ is

$$
\operatorname{Ind}^{\Gamma}(X):=\operatorname{Ind}^{\Gamma}\left([X]^{\Gamma}\right)
$$

where $\operatorname{Ind}^{\Gamma}=\left(\mathrm{ev}_{1}\right)_{*}: \mathrm{K}_{n}^{\Gamma}(X)=\mathrm{K}_{n}\left(\mathrm{C}_{\Gamma}^{*} \mathrm{~L}(X)\right) \rightarrow \mathrm{K}_{n}\left(\mathrm{C}_{\Gamma}^{*}(X)\right)$.

More generally, for a $\Gamma$-invariant subset $Z \subseteq X$, the (equivariant) coarse index modulo $Z$ is defined by

$$
\operatorname{Ind}_{/ / Z}^{\Gamma}(X):=\operatorname{Ind}_{/ / Z}^{\Gamma}\left([X]^{\Gamma}\right) \in \mathrm{K}_{*}\left(\frac{\mathrm{C}_{\Gamma}^{*}(X)}{\mathrm{C}_{\Gamma}^{*}(Z \subset X)}\right)
$$


where we use the map $\operatorname{Ind}_{/ / Z}^{\Gamma}$ from Corollary 2.1.4.

Remark 2.2.3. The fundamental class (and hence the coarse index) does not depend on the choice of the Riemannian metric. In our framework, this will become apparent in Proposition 2.2.11.

Remark 2.2.4 ([QR10, Section 4], [Dum05, Section 3]). There is an asymptotic morphism $\gamma: \mathrm{C}_{\Gamma}^{*} \mathrm{~L}(X) \widehat{\otimes} \mathcal{C}_{0}(X) \rightarrow \mathbb{K}, \gamma_{t}(L \widehat{\otimes} f)=L(t) f$. Using a suitable product in E-theory, this induces a map $\gamma_{*}: \mathrm{K}_{*}\left(\mathrm{C}_{\Gamma}^{*} \mathrm{~L}(X)\right) \rightarrow \mathrm{K}_{\Gamma}^{-*}\left(\mathcal{C}_{0}(X)\right)=\mathrm{K}_{*}^{\Gamma}(X)$. The class $\gamma_{*}\left([X]^{\Gamma}\right)$ is represented by the $\Gamma$-equivariant asymptotic morphism $\alpha: \mathcal{S} \widehat{\otimes} \mathcal{C}_{0}(X) \rightarrow-\rightarrow \mathbb{K}\left(\mathrm{L}^{2}\left(\phi_{g}\right)\right) \cong$ $\mathbb{K} \widehat{\otimes} \mathrm{Cl}_{n}, \alpha_{t}(f \widehat{\otimes} g)=f\left(\frac{1}{t} \mathscr{D}_{g}\right) g$. Specializing to $X=\mathbb{R}^{n}$, we observe that $\gamma_{*}\left(\left[\mathbb{R}^{n}\right]\right)$ is precisely the Dirac element from Subsection 1.1.3.

\subsubsection{The partial $\rho$-invariant and the localized coarse index}

In addition to the setup of the previous subsection, let $Z \subseteq X$ be some $\Gamma$-invariant subset. We will now define secondary invariants associated to Riemannian metrics $g \in \mathcal{R}_{Z}^{+}(X)^{\Gamma}$. We will use the following analytic fact.

Lemma 2.2.5 ([Roe16, Lemma 2.3],[HPS15, Proposition 3.15]). Let $g \in \mathcal{R}_{Z}^{+}(X)^{\Gamma}$. Choose $\varepsilon>0$ such that $\operatorname{scal}_{g}(x)>4 \varepsilon^{2}$ for all $x \in X \backslash Z$. Then $\left(\operatorname{ev}_{1} \circ \alpha_{g}\right)(f)=f\left(\not{D}_{g}\right) \in$ $\mathrm{C}_{\Gamma}^{*}\left(Z \subset X ; \mathrm{Cl}_{n}\right)$ for all $f \in \mathcal{S}(-\varepsilon, \varepsilon)$.

In other words, the restriction of $\alpha_{g}$ to $\mathcal{S}(-\varepsilon, \varepsilon)$ takes values in $\mathrm{C}_{\Gamma}^{*} \mathrm{~L}_{Z}\left(X ; \mathrm{Cl}_{n}\right)$. Since the inclusion of $\mathcal{S}(-\varepsilon, \varepsilon) \hookrightarrow \mathcal{S}$ is a homotopy equivalence, this means that the fundamental class $[X]^{\Gamma}$ can be lifted to an element of $\mathrm{K}_{n}\left(\mathrm{C}_{\Gamma}^{*} \mathrm{~L}_{Z}(X)\right)$. To make this precise, we fix a graded $*$-homomorphism $\psi: \mathcal{S} \rightarrow \mathcal{S}(-\varepsilon, \varepsilon)$ which is homotopy inverse to the inclusion $\mathcal{S}(-\varepsilon, \varepsilon) \hookrightarrow \mathcal{S}$, see Lemma 1.1.4.

Definition 2.2.6. The equivariant partial $\rho$-invariant of $g \in \mathcal{R}_{Z}^{+}(X)$ is defined as follows:

$$
\rho_{Z}^{\Gamma}(g):=\left[\alpha_{g} \circ \psi\right] \in \mathrm{K}_{0}\left(\mathrm{C}_{\Gamma}^{*} \mathrm{~L}_{Z}\left(X ; \mathrm{Cl}_{n}\right)\right)=\mathrm{K}_{n}\left(\mathrm{C}_{\Gamma}^{*} \mathrm{~L}_{Z}(X)\right)=\mathrm{S}_{n}^{\Gamma}(X / / Z),
$$

where $\psi: \mathcal{S} \rightarrow \mathcal{S}(-\varepsilon, \varepsilon)$ and $\varepsilon>0$ are chosen as above.

For $Z=\emptyset$ we write $\rho^{\Gamma}(g) \in \mathrm{S}_{n}^{\Gamma}(X)$.

Here we have included $g$ in the notation of the partial secondary invariant to emphasize that this class may indeed depend on the metric. However, since $\psi$ is unique up to homotopy as a homotopy inverse to the inclusion $\mathcal{S}(-\varepsilon, \varepsilon) \hookrightarrow \mathcal{S}$, the partial $\rho$-invariant $\rho_{Z}^{\Gamma}(g)$ does not depend on the particular choices of $\varepsilon$ and $\psi$.

The following proposition is immediate from the construction:

Proposition 2.2.7. Let $Z \subseteq B \subseteq X$ be $\Gamma$-invariant subsets and $g \in \mathcal{R}_{Z}^{+}(X)^{\Gamma} \subseteq$ $\mathcal{R}_{B}^{+}(X)^{\Gamma}$. Using the notation from Subsection 2.1.1, the following equality holds:

$$
q_{Z, B}^{\Gamma}\left(\rho_{Z}^{\Gamma}(g)\right)=\rho_{B}^{\Gamma}(g) .
$$

In particular, we have $q_{Z, X}^{\Gamma}\left(\rho_{Z}^{\Gamma}(g)\right)=[X]^{\Gamma}$ and $\rho_{X}^{\Gamma}(g)=[X]^{\Gamma}$. 
By the exact sequence from Corollary 2.1.4, we conclude:

Corollary 2.2.8. If $\operatorname{Ind}_{/ / Z}^{\Gamma}(X) \neq 0$, then $\mathcal{R}_{Z}^{+}(X)^{\Gamma}$ is empty.

Definition 2.2.9. Let $g \in \mathcal{R}_{Z}^{+}(X)^{\Gamma}$. The (equivariant) localized coarse index of $(X, g)$ is defined as follows:

$$
\operatorname{Ind}_{Z}^{\Gamma}(X, g):=\operatorname{Ind}_{Z}^{\Gamma}\left(\rho_{Z}^{\Gamma}(g)\right) \in \mathrm{K}_{n}\left(\mathrm{C}_{\Gamma}^{*}(Z)\right) .
$$

Moreover, if $A \subseteq Z$ is another $\Gamma$-invariant subset, then we define the (equivariant) localized coarse index modulo $A$ as

$$
\operatorname{Ind}_{Z / / A}^{\Gamma}(X, g):=\operatorname{Ind}_{Z / / A}^{\Gamma}\left(\rho_{Z}^{\Gamma}(g)\right) \in \mathrm{K}_{n}\left(\frac{\mathrm{C}_{\Gamma}^{*}(Z)}{\mathrm{C}_{\Gamma}^{*}(A \subset Z)}\right)
$$

Here we have again used the $\operatorname{maps} \operatorname{Ind}_{Z}^{\Gamma}$ and $\operatorname{Ind}_{Z / / A}^{\Gamma}$ from Corollary 2.1.4 and Proposition 2.1.3.

This localized coarse index has been previously introduced and studied by Roe, see [Roe96, Proposition 3.11] and [Roe16].

Remark 2.2.10. If the $\Gamma$-action on $X$ is cocompact, then any non-empty $\Gamma$-invariant subset $Z$ is coarsely equivalent to $X$ and so $\mathrm{S}_{*}^{\Gamma}(X / / Z)=\mathrm{K}_{*}^{\Gamma}(X)$ for all $Z \neq \emptyset$. Moreover, note that it is a consequence of the Kazdan-Warner theorem [KW75] that on a closed manifold (such as $\Gamma \backslash X$ if the action is cocompact) of dimension $\geqslant 3$ there are no obstructions to positive scalar curvature outside a subset of non-empty interior. We conclude that partial secondary invariants for subsets other than $Z=X$ or $Z=\emptyset$ are only interesting if $X$ is not $\Gamma$-cocompact.

The partial $\rho$-invariant is a concordance invariant in the following sense:

Proposition 2.2.11. Let $g_{0}, g_{1} \in \mathcal{R}_{Z}^{+}(X)^{\Gamma}$ be concordant relative to $Z$ as in Definition 1.3.5. Then $\rho_{Z}^{\Gamma}\left(g_{0}\right)=\rho_{Z}^{\Gamma}\left(g_{1}\right)$.

We defer the proof of Proposition 2.2.11 to Subsection 2.4.1, where it will be a consequence of the partitioned manifold index theorem for partial $\rho$-invariants.

We deduce the following result (see Remark 1.3.6):

Corollary 2.2.12. Let $g_{0}, g_{1} \in \mathcal{R}_{Z}^{+}(X)^{\Gamma}$ be equal on $X \backslash Z$. Then $\rho_{Z}^{\Gamma}\left(g_{0}\right)=\rho_{Z}^{\Gamma}\left(g_{1}\right)$.

Under the hypotheses of Proposition 2.2.11 (respectively Corollary 2.2.12), we also have $\operatorname{Ind}_{Z}^{\Gamma}\left(X, g_{0}\right)=\operatorname{Ind}_{Z}^{\Gamma}\left(X, g_{1}\right)$. Moreover, if $A \subseteq Z$ and $g \in \mathcal{R}_{A}^{+}(X)^{\Gamma} \subseteq$ $\mathcal{R}_{Z}^{+}(X)^{\Gamma}$, then $\operatorname{Ind}_{Z / / A}^{\Gamma}(X, g)$ vanishes, as follows from exactness of the sequence in Proposition 2.1.3. We deduce:

Corollary 2.2.13. Let $g \in \mathcal{R}_{Z}^{+}(X)^{\Gamma}$ with $\operatorname{Ind}_{Z / / A}^{\Gamma}(X, g) \neq 0$. Then $g$ is not concordant relative to $Z$ to an element of $\mathcal{R}_{A}^{+}(X)^{\Gamma}$. 


\subsubsection{The coarse APS-index for partial psc at the boundary}

In the work of Piazza-Schick [PS14] and Xie-Yu [XY14b], a crucial role falls upon a "higher Atiyah-Patodi-Singer index" for manifolds with positive scalar curvature at the boundary. In these articles, its construction is based on Roe's localized coarse index. This is also the approach that we will take here. A discussion that this agrees with other constructions of this APS-type index can be found in [PS14, Subsection $2.2]$. Following our pattern of generalizing all constructions to partial positive scalar curvature, we will construct a coarse APS-index for positive scalar curvature on the boundary outside a prescribed subset.

In this subsection, we fix $Y$ to be an $(n+1)$-dimensional complete spin manifold endowed with a $\Gamma$-action and with boundary $\partial Y=X$. Moreover, we suppose that $Y$ has finite Hausdorff distance to its boundary, that is, $\sup _{y \in Y} d_{Y}(y, X)<\infty$. In other words, the inclusion $X \hookrightarrow Y$ is a coarse equivalence.

We will freely use the conventions from Subsection 1.3.3. In particular, let $Y_{\infty}$ be the spin manifold obtained from $Y$ by attaching a cylindrical end $X \times \mathbb{R}_{\geqslant 0}$.

Let $Z \subseteq X$ be some $\Gamma$-invariant subset and define $Z_{\infty}:=Y \cup\left(Z \times \mathbb{R}_{\geqslant 0}\right) \subseteq Y_{\infty}$.

Lemma 2.2.14. The inclusions $X \hookrightarrow Y \hookrightarrow Y_{\infty}$ induce isomorphisms as follows:

$$
\mathrm{K}_{*}\left(\frac{\mathrm{C}_{\Gamma}^{*}(X)}{\mathrm{C}_{\Gamma}^{*}(Z \subset X)}\right) \cong \mathrm{K}_{*}\left(\frac{\mathrm{C}_{\Gamma}^{*}(Y)}{\mathrm{C}_{\Gamma}^{*}(Z \subset Y)}\right) \cong \mathrm{K}_{*}\left(\frac{\mathrm{C}_{\Gamma}^{*}\left(Z_{\infty} \subset Y_{\infty}\right)}{\mathrm{C}_{\Gamma}^{*}\left(Z \times \mathbb{R}_{\geqslant 0} \subset Y_{\infty}\right)}\right) .
$$

Proof. The first isomorphism is a consequence of the assumption that the inclusion $X \hookrightarrow Y$ is a coarse equivalence. For the second isomorphism, it suffices to show that

$$
\begin{gathered}
\mathrm{C}_{\Gamma}^{*}\left(Z_{\infty} \subset Y_{\infty}\right)=\mathrm{C}_{\Gamma}^{*}\left(Y \subset Y_{\infty}\right)+\mathrm{C}_{\Gamma}^{*}\left(Z \times \mathbb{R}_{\geqslant 0} \subset Y_{\infty}\right), \\
\mathrm{C}_{\Gamma}^{*}\left(Y \subset Y_{\infty}\right) \cap \mathrm{C}_{\Gamma}^{*}\left(Z \times \mathbb{R}_{\geqslant 0} \subset Y_{\infty}\right)=\mathrm{C}_{\Gamma}^{*}\left(Z \subset Y_{\infty}\right) .
\end{gathered}
$$

This follows from Lemma 2.1.6.

Denote the inverse of the isomorphism from Lemma 2.2.14 by

$$
\zeta: \mathrm{K}_{*}\left(\frac{\mathrm{C}_{\Gamma}^{*}\left(Z_{\infty}\right)}{\mathrm{C}_{\Gamma}^{*}\left(Z \times \mathbb{R}_{\geqslant 0} \subset Z_{\infty}\right)}\right) \rightarrow \mathrm{K}_{*}\left(\frac{\mathrm{C}_{\Gamma}^{*}(X)}{\mathrm{C}_{\Gamma}^{*}(Z \subset X)}\right) .
$$

Definition 2.2.15. Let $g \in \mathcal{R}_{Z}^{+}(X)^{\Gamma}$. Choose an arbitrary $h \in \mathcal{R}_{Z}^{+}(Y)^{\Gamma}$ with $\partial h=g$. We then have $h_{\infty} \in \mathcal{R}_{Z_{\infty}}^{+}\left(Y_{\infty}\right)$. We define the coarse APS-index of $Y$ with respect to $g \in \mathcal{R}_{Z}^{+}(X)^{\Gamma}$ by

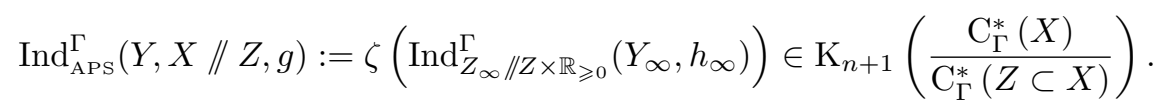

Remark 2.2.16. If $Z=\emptyset$, this yields an index $\operatorname{Ind}_{\mathrm{APS}}^{\Gamma}(Y, X, g) \in \mathrm{K}_{n+1}\left(\mathrm{C}_{\Gamma}^{*}(X)\right)$ for all $g \in \mathcal{R}^{+}(X)^{\Gamma}$; this is the index that is studied in [PS14; XY14b]. 
Remark 2.2.17. Concordance invariance (Proposition 2.2.11) implies that the coarse APS-index $\operatorname{Ind}_{\text {APS }}^{\Gamma}(Y, X / / Z, g)$ does not depend on the choice of the particular $h \in$ $\mathcal{R}_{Z}^{+}(Y)^{\Gamma}$ with $\partial h=g$. This is the case because $Z_{\infty} \supseteq Y$ and $\operatorname{Ind}_{Z_{\infty} / / Z \times \mathbb{R}_{\geqslant 0}}^{\Gamma}\left(Y_{\infty}, h_{\infty}\right)$ does not depend on the values of $h$ on $Z_{\infty}$, see Corollary 2.2.12.

Remark 2.2.18. This construction also yields an APS-index if we do not make any psc assumptions whatsoever on the boundary (that is, take $Z=X$ ). However, in this case, the receptacle of the index is the zero group $\mathrm{K}_{*}\left(\mathrm{C}_{\Gamma}^{*}(X) / \mathrm{C}_{\Gamma}^{*}(X)\right)$ and hence contains no information.

The following proposition says that non-vanishing of the APS-index is an obstruction to the existence of extensions of $g$ to $Y$ which have positive scalar curvature outside a subset that is coarsely equivalent to $Z$.

Proposition 2.2.19. Let $g \in \mathcal{R}_{Z}^{+}(X)^{\Gamma}$. Suppose that there exists a $\Gamma$-invariant subset $A \subseteq Y$ such that $\sup _{z \in Z} d(z, A)<\infty$ and $h \in \mathcal{R}_{A}^{+}(Y)^{\Gamma}$ with $\partial h=g$. Then $\operatorname{Ind}_{\mathrm{APS}}^{\Gamma}(Y, X / / Z, g)=0$.

Proof. Let $A_{\infty}:=A \cup\left(Z \times \mathbb{R}_{\geqslant 0}\right)$. Then $\operatorname{Ind}_{Z_{\infty}}^{\Gamma}\left(Y_{\infty}, h_{\infty}\right) \in \mathrm{K}_{*}\left(\mathrm{C}_{\Gamma}^{*}\left(Z_{\infty} \subset Y_{\infty}\right)\right)$ can be lifted to $\operatorname{Ind}_{A_{\infty}}^{\Gamma}\left(Y_{\infty}, h_{\infty}\right) \in \mathrm{K}_{*}\left(\mathrm{C}_{\Gamma}^{*}\left(A_{\infty} \subset Y_{\infty}\right)\right)$. The assumption on $A$ implies $\mathrm{C}_{\Gamma}^{*}\left(A_{\infty} \subset Y_{\infty}\right)=\mathrm{C}_{\Gamma}^{*}\left(Z \times \mathbb{R}_{\geqslant 0} \subset Y_{\infty}\right)$. Hence the image of $\operatorname{Ind}_{Z_{\infty}}^{\Gamma}\left(Y_{\infty}, h_{\infty}\right)$ in $\mathrm{K}_{*}\left(\mathrm{C}_{\Gamma}^{*}\left(Z_{\infty} \subset Y_{\infty}\right) / \mathrm{C}_{\Gamma}^{*}\left(\left(Z \times \mathbb{R}_{\geqslant 0}\right) \subset Y_{\infty}\right)\right)$ vanishes. Consequently, by the definition of the coarse APS-index, $\operatorname{Ind}_{\mathrm{APS}}^{\Gamma}(Y, X / / Z, g)$ also vanishes.

Corollary 2.2.20 $(Z=\emptyset)$. Let $g \in \mathcal{R}^{+}(X)^{\Gamma}$. If $\operatorname{Ind}_{\mathrm{APS}}^{\Gamma}(Y, X, g) \neq 0$, then there exists no $h \in \mathcal{R}^{+}(Y)^{\Gamma}$ with $\partial h=g$.

\subsubsection{The index difference}

Hitchin [Hit74] constructed a secondary index invariant (taking values in $\mathrm{KO}^{-n-1}(*)$ ) associated to two positive scalar curvature metrics. Variants of this "index difference" or "relative index" have been used extensively in the recent literature to study different topological aspects of the space of positive scalar curvature metrics, see for example [HSS14; BER14; XY14a; WY13; XY13]. In this subsection, we briefly present the construction of such an invariant by defining a "coarse index difference" of two Riemannian metrics in $\mathcal{R}_{Z}^{+}(X)$ based on the coarse APS-index from the previous subsection. The non-vanishing of this invariant is an obstruction to concordance relative to $Z$.

Definition 2.2.21. Let $g_{0}, g_{1} \in \mathcal{R}_{Z}^{+}(X)$. The index difference of $g_{0}$ and $g_{1}$ is

$$
\begin{array}{r}
\operatorname{Ind}_{\mathrm{diff}}^{\Gamma}\left(g_{0}, g_{1} / / Z\right):=\left(\operatorname{pr}_{X}\right)_{*} \operatorname{Ind}_{\mathrm{APS}}^{\Gamma}\left(X \times[0,1], X \times\{0,1\} / / Z \times\{0,1\}, g_{0} \sqcup g_{1}\right) \\
\in \mathrm{K}_{n+1}\left(\frac{\mathrm{C}_{\Gamma}^{*}(X)}{\mathrm{C}_{\Gamma}^{*}(Z \subset X)}\right),
\end{array}
$$

where $\operatorname{pr}_{X}: X \times\{0,1\} \rightarrow X$ is the projection onto the first coordinate.

In the case of $Z=\emptyset$, we have $\operatorname{Ind}_{\text {diff }}^{\Gamma}\left(g_{0}, g_{1}\right) \in \mathrm{K}_{n+1}\left(\mathrm{C}_{\Gamma}^{*}(X)\right)$. 
Corollary 2.2.22. If $g_{0}$ and $g_{1}$ are concordant relative to $Z$, then the index difference $\operatorname{Ind}_{\text {diff }}^{\Gamma}\left(g_{0}, g_{1} / / Z\right)$ vanishes.

Proof. The Riemannian metrics $g_{0}$ and $g_{1}$ are concordant relative to $Z$ if and only if there exists an extension of $g_{0} \sqcup g_{1}$ to a Riemannian metric $h \in \mathcal{R}_{Z \times[0,1]}^{+}(X \times[0,1])^{\Gamma}$. Therefore, the statement follows from Proposition 2.2.19.

\subsection{Product formulas for secondary invariants}

Suppose that $X_{i}, i \in\{1,2\}$, are $n_{i}$-dimensional complete spin manifolds endowed with $\Gamma_{i}$-actions. Their product $X=X_{1} \times X_{2}$ is an $n:=n_{1}+n_{2}$-dimensional spin manifold.

Fix Riemannian metrics $g_{i} \in \mathcal{R}\left(X_{i}\right)^{\Gamma}, i \in\{1,2\}$ and set $g:=g_{1} \oplus g_{2} \in \mathcal{R}(X)^{\Gamma}$. A principal $\operatorname{Spin}(n)$-bundle covering the $\mathrm{SO}(n)$-frame bundle of $X$ may be obtained as the bundle associated to $\mathrm{P}_{\text {Spin }}\left(X_{1}\right) \times_{\mathbb{Z}_{2}} \mathrm{P}_{\text {Spin }}\left(X_{2}\right)$ via the inclusion $\operatorname{Spin}\left(n_{1}\right) \times_{\mathbb{Z}_{2}} \operatorname{Spin}\left(n_{2}\right) \hookrightarrow$ $\operatorname{Spin}(n)$. In view of the isomorphism $\mathrm{Cl}_{n_{1}} \widehat{\otimes} \mathrm{Cl}_{n_{2}} \cong \mathrm{Cl}_{n}$, we may identify the Cl-spinor bundles as follows: $\Phi_{g}=\operatorname{pr}_{1}^{*} \Phi_{g_{1}} \widehat{\otimes} \mathrm{pr}_{2}^{*} \Phi_{g_{1}}$, where $\mathrm{pr}_{i}: X_{1} \times X_{2} \rightarrow X_{i}$ are the canonical projection maps. On the level of $\mathrm{L}^{2}$-sections, this means that we may identify $\mathrm{L}^{2}\left(X_{1}, \Phi_{g_{1}}\right) \widehat{\otimes} \mathrm{L}^{2}\left(X_{2}, \Phi_{g_{2}}\right)=\mathrm{L}^{2}\left(X, \Phi_{g}\right)$. Hence we can use the description of the external product from Subsection 2.1.4 in this context. The Cl-linear Dirac operators $\mathscr{D}_{g_{i}}$ and $\mathscr{D}_{g}$ on $\mathscr{\Phi}_{g_{i}}$ and $\mathscr{\Phi}_{g}, i \in\{1,2\}$, respectively, satisfy the relation $\mathscr{D}_{g}=\mathscr{D}_{g_{1}} \widehat{\otimes} \mathrm{id}+\mathrm{id} \widehat{\otimes} \mathfrak{D}_{g_{2}}$.

Theorem 2.3.1. For $i \in\{1,2\}$, let $X_{i}$ be a complete spin manifold endowed with a free and proper action of a discrete group $\Gamma_{i}$. Let $Z_{1} \subseteq X_{1}$ be some $\Gamma_{1}$-invariant subset. Set $X:=X_{1} \times X_{2}$ and $Z:=Z_{1} \times X_{2}$ and $\Gamma:=\Gamma_{1} \times \Gamma_{2}$.

Then for all $g_{1} \in \mathcal{R}_{Z_{1}}^{+}\left(X_{1}\right)^{\Gamma_{1}}$ and $g_{2} \in \mathcal{R}\left(X_{2}\right)^{\Gamma_{2}}$ with $g:=g_{1} \oplus g_{2} \in \mathcal{R}_{Z}^{+}(X)$, the following product formula holds:

$$
\rho_{Z}^{\Gamma}(g)=\rho_{Z_{1}}^{\Gamma_{1}}\left(g_{1}\right) \otimes\left[X_{2}\right]^{\Gamma_{2}} .
$$

The external product we us here has been defined in Subsection 2.1.4. Before turning to the proof, we discuss two straightforward corollaries. Firstly, by setting $Z_{1}=X_{1}$ or $Z_{1}=\emptyset$, we deduce:

Corollary 2.3.2. In the situation of Theorem 2.3 .1

$$
[X]^{\Gamma}=\left[X_{1}\right]^{\Gamma_{1}} \otimes\left[X_{2}\right]^{\Gamma_{2}} .
$$

If $g_{1} \in \mathcal{R}^{+}\left(X_{1}\right)^{\Gamma_{1}}$ and $g_{2} \in \mathcal{R}\left(X_{2}\right)^{\Gamma_{2}}$ with $g:=g_{1} \oplus g_{2} \in \mathcal{R}^{+}(X)^{\Gamma}$, then

$$
\rho^{\Gamma}(g)=\rho^{\Gamma_{1}}\left(g_{1}\right) \otimes\left[X_{2}\right]^{\Gamma_{2}} .
$$

Secondly, applying the index map to (2.3.1) yields:

Corollary 2.3.3. In the setup of Theorem 2.3.1, the product formula for the localized index

$$
\operatorname{Ind}_{Z}^{\Gamma}(X, g)=\operatorname{Ind}_{Z_{1}}^{\Gamma_{1}}\left(X_{1}, g_{1}\right) \otimes \operatorname{Ind}^{\Gamma_{2}}\left(X_{2}\right)
$$

holds for all $g_{1} \in \mathcal{R}_{Z_{1}}^{+}\left(X_{1}\right)^{\Gamma_{1}}$ and $g_{2} \in \mathcal{R}\left(X_{2}\right)^{\Gamma_{2}}$ with $g:=g_{1} \oplus g_{2} \in \mathcal{R}_{Z}^{+}(X)$. 
The main part of the proof of Theorem 2.3.1 consists of some standard computations which we indicate in the following two lemmas for the convenience of the reader.

Lemma 2.3.4 ([HKT98, Appendix A.4]). Let $D_{i}: \mathcal{H}_{i} \supseteq \operatorname{dom}\left(D_{i}\right) \rightarrow \mathcal{H}_{i}$ be odd, (unbounded) self-adjoint operators on graded Hilbert spaces $\mathcal{H}_{i}, i \in\{1,2\}$. Then we have the equality (of bounded operators on $\mathcal{H}_{1} \widehat{\otimes} \mathcal{H}_{2}$ ),

$$
\exp \left(-\left(D_{1} \widehat{\otimes} \mathrm{id}+\mathrm{id} \widehat{\otimes} D_{2}\right)^{2}\right)=\exp \left(-D_{1}^{2}\right) \widehat{\otimes} \exp \left(-D_{2}^{2}\right) .
$$

Proof. If both operators $D_{1}$ and $D_{2}$ are bounded, then the result follows immediately by the functional equation for the exponential function. The general case can be reduced to the bounded case using the spectral theorem and an approximation argument.

Lemma 2.3.5. In the setup of Theorem 2.3.1 the following diagram commutes:

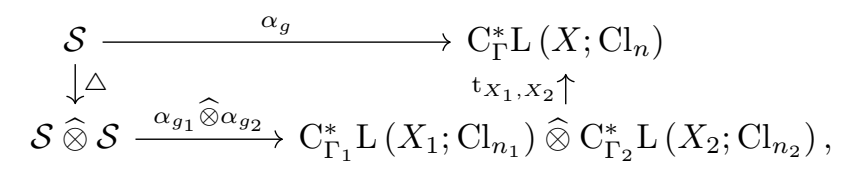

where the $*$-homomorphisms $\alpha_{g}, \alpha_{g_{i}}$ are defined according to (2.2.1).

Proof. The statement and proof are essentially the same as, for example, in the proof of [Dum05, Theorem 4.1]. It suffices to check commutativity for the generators $\mathrm{e}^{-\mathrm{x}^{2}}$ and $\mathrm{xe}^{-\mathrm{x}^{2}}$ of $\mathcal{S}$. Indeed, we have $\alpha_{g}\left(\mathrm{e}^{-\mathrm{x}^{2}}\right)(t)=\mathrm{e}^{-\frac{1}{t^{2}} \not_{g}^{2}}=\mathrm{e}^{-\left(t^{-1} \not_{g_{1}} \widehat{\otimes} 1+1 \widehat{\otimes} t^{-1} \not_{g_{2}}\right)^{2}}$ and $\mathrm{t}_{X_{1}, X_{2}}\left(\alpha_{g_{1}} \widehat{\otimes} \alpha_{g_{2}}\left(\triangle\left(\mathrm{e}^{-\mathrm{x}^{2}}\right)\right)\right)(t)=\mathrm{e}^{-\frac{1}{t^{2}} \not{D}_{g_{1}}^{2}} \widehat{\otimes} \mathrm{e}^{-\frac{1}{t^{2}} \not_{g_{2}}^{2}}$. Thus Lemma 2.3.4 with $D_{i}=\frac{1}{t} \not_{g_{i}}$ implies that $\alpha_{g}\left(\mathrm{e}^{-\mathrm{x}^{2}}\right)=\mathrm{t}_{X_{1}, X_{2}}\left(\alpha_{g_{2}} \widehat{\otimes} \alpha_{g_{2}}\left(\triangle\left(\mathrm{e}^{-\mathrm{x}^{2}}\right)\right)\right)$, as required. A similar computation shows commutativity on the generator $\mathrm{xe}^{-\mathrm{x}^{2}}$.

Proof of Theorem 2.3.1. The assumptions allow to find $\varepsilon>0$ such that $\alpha_{g_{1}}$ maps $\mathcal{S}(-\varepsilon, \varepsilon)$ to $\mathrm{C}_{\Gamma_{1}}^{*} \mathrm{~L}_{Z_{1}}\left(X_{1} ; \mathrm{Cl}_{n_{1}}\right)$ and $\alpha_{g}$ maps $\mathcal{S}(-\varepsilon, \varepsilon)$ to $\mathrm{C}_{\Gamma}^{*} \mathrm{~L}_{Z}\left(X ; \mathrm{Cl}_{n}\right)$. Choose a graded $*$-homomorphism $\psi: \mathcal{S} \rightarrow \mathcal{S}(-\varepsilon, \varepsilon)$ that is homotopy inverse to the inclusion $\iota: \mathcal{S}(-\varepsilon, \varepsilon) \hookrightarrow \mathcal{S}$ (as in Definition 2.2.6).

We then have $\rho_{Z}^{\Gamma}(g)=\left[\alpha_{g} \circ \psi\right] \in \mathrm{K}_{0}\left(\mathrm{C}_{\Gamma}^{*} \mathrm{~L}_{Z}\left(X ; \mathrm{Cl}_{n}\right)\right.$ and $\rho_{Z_{1}}^{\Gamma_{1}}\left(g_{1}\right)=\left[\alpha_{g_{1}} \circ \psi\right] \in$ $\mathrm{K}_{0}\left(\mathrm{C}_{\Gamma_{1}}^{*} \mathrm{~L}_{Z_{1}}\left(X_{1} ; \mathrm{Cl}_{n_{1}}\right)\right)$ and $\left[X_{2}\right]^{\Gamma_{2}}=\left[\alpha_{g_{2}}\right]=\left[\alpha_{g_{2}} \circ \psi\right] \in \mathrm{K}_{0}\left(\mathrm{C}_{\Gamma_{2}}^{*} \mathrm{~L}\left(X_{2} ; \mathrm{Cl}_{n_{2}}\right)\right)$. Thus, to prove the product formula, we need to show that the following diagram commutes up to homotopy:

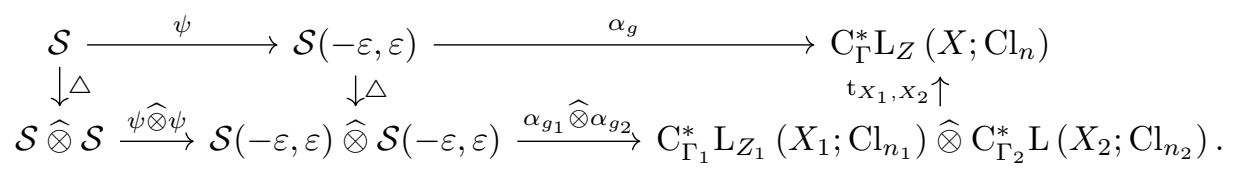

Indeed, the left square commutes up to homotopy by Corollary 1.1.5. The right square strictly commutes as it is a restriction of the diagram from Lemma 2.3.5. 
Corollary 2.3.6. In addition to the setup of Theorem 2.3.1, let $X_{1}=\partial Y_{1}$, where $Y_{1}$ is a complete spin manifold endowed with a $\Gamma_{2}$-action. Set $Y:=Y_{1} \times X_{2}$, then $\partial Y=X_{1} \times X_{2}=X$.

Then for all $g_{1} \in \mathcal{R}_{Z_{1}}^{+}\left(X_{1}\right)^{\Gamma_{1}}$ and $g_{2} \in \mathcal{R}\left(X_{2}\right)^{\Gamma_{2}}$ with $g:=g_{1} \oplus g_{2} \in \mathcal{R}_{Z}^{+}(X)$, the following product formula holds:

$$
\operatorname{Ind}_{\mathrm{APS}}^{\Gamma}(Y, X / / Z, g)=\operatorname{Ind}_{\mathrm{APS}}^{\Gamma_{1}}\left(Y_{1}, X_{1} / / Z_{1}, g_{1}\right) \otimes \operatorname{Ind}^{\Gamma_{2}}\left(X_{2}\right),
$$

where the external product (2.1.8) is used.

Proof. We have a canonical identification

$$
\begin{aligned}
Y_{\infty} & =Y \cup_{X}\left(X \times \mathbb{R}_{\geqslant 0}\right)=\left(Y_{1} \times X_{2}\right) \cup_{X}\left(X_{1} \times X_{2} \times \mathbb{R}_{\geqslant 0}\right) \\
& =\left(Y_{1} \cup_{X_{1}}\left(X_{1} \times \mathbb{R}_{\geqslant 0}\right)\right) \times X_{2} \\
& =\left(Y_{1}\right)_{\infty} \times X_{2} .
\end{aligned}
$$

Let $Z_{\infty}:=Y \cup\left(Z \times \mathbb{R}_{\geqslant 0}\right)$ and $\left(Z_{1}\right)_{\infty}:=Y_{1} \cup\left(Z_{1} \times \mathbb{R}_{\geqslant 0}\right)$ as in Subsection 2.2.3. Then $Z_{\infty}=\left(Z_{1}\right)_{\infty} \times X_{2}$ under the above identification. Let $h_{1} \in \mathcal{R}\left(Y_{1}\right)^{\Gamma_{1}}$ with $\partial h_{1}=g_{1}$ and set $h:=h_{1} \oplus g_{2} \in \mathcal{R}(Y)^{\Gamma}$. Then $\left(h_{1}\right)_{\infty}:=h_{1} \cup_{X_{1}}\left(g_{1} \oplus \mathrm{d} t^{2}\right) \in \mathcal{R}_{\left(Z_{1}\right)_{\infty}}^{+}\left(X_{1}\right)^{\Gamma_{1}}$ and $h_{\infty}:=h \cup_{X}\left(g \oplus \mathrm{d} t^{2}\right)=\left(h_{1}\right)_{\infty} \oplus g_{2} \in \mathcal{R}_{Z_{\infty}}^{+}\left(Y_{\infty}\right)^{\Gamma}$. Corollary 2.3 .3 implies that

$$
\begin{aligned}
\operatorname{Ind}_{Z_{\infty}}^{\Gamma}\left(Y_{\infty}, h_{\infty}\right)=\operatorname{Ind}_{\left(Z_{1}\right)_{\infty}}^{\Gamma}\left(\left(Y_{1}\right)_{\infty},\left(h_{1}\right)_{\infty}\right) \otimes & \operatorname{Ind}^{\Gamma_{2}}\left(X_{2}\right) \\
& \in \mathrm{K}_{n+1}\left(\mathrm{C}_{\Gamma}^{*}\left(Z_{\infty} \subset Y_{\infty}\right)\right) .
\end{aligned}
$$

Then $\operatorname{Ind}_{\mathrm{APS}}^{\Gamma}(Y, X / / Z, g)$ and $\operatorname{Ind}_{\mathrm{APS}}^{\Gamma_{1}}\left(Y_{1}, X_{1} / / Z_{1}, g_{1}\right)$ are defined by applying the isomorphism from Lemma 2.2.14 to $\operatorname{Ind}_{Z_{\infty} / / Z \times \mathbb{R}_{\geqslant 0}}\left(Y_{\infty}, h_{\infty}\right)$ and $\operatorname{Ind}_{\left(Z_{1}\right)_{\infty} / / Z_{1} \times \mathbb{R}_{\geqslant 0}}\left(\left(Y_{1}\right)_{\infty},\left(h_{1}\right)_{\infty}\right)$, respectively. Therefore, we can deduce (2.3.6) from (2.3.7) using naturality of the external product (2.1.8).

Corollary 2.3.7. In the setup of Theorem 2.3.1, let $g_{X_{1}, 0}, g_{X_{1}, 1} \in \mathcal{R}_{Z_{1}}^{+}\left(X_{1}\right)^{\Gamma_{1}}$ such that $g_{X, j}:=g_{X_{1}, j} \oplus g_{2} \in \mathcal{R}_{Z}^{+}(X)^{\Gamma}$ for $j \in\{0,1\}$. Then:

$$
\operatorname{Ind}_{\text {diff }}^{\Gamma}\left(g_{X, 1}, g_{X, 0} / / Z\right)=\operatorname{Ind}_{\text {diff }}^{\Gamma_{1}}\left(g_{X_{1}, 1}, g_{X_{1}, 0} / / Z_{1}\right) \otimes \operatorname{Ind}^{\Gamma_{2}}\left(X_{2}\right) .
$$

Proof. The statement follows from Corollary 2.3 .6 by applying it to $Y_{1}=X_{1} \times[0,1]$.

\subsection{1 "Boundary of Dirac is Dirac"}

The following lemma is a standard fact. However, we include a proof so as to demonstrate that it can be verified directly in our present setup.

Lemma 2.3.8. The boundary map $\partial_{\mathrm{MV}}: \mathrm{K}_{1}\left(\mathrm{C}^{*} \mathrm{~L}(\mathbb{R})\right) \rightarrow \mathrm{K}_{0}\left(\mathrm{C}^{*} \mathrm{~L}(\{0\})\right)$ associated to the Mayer-Vietoris sequence of the cover $\mathbb{R}=\mathbb{R}_{\geqslant 0} \cup \mathbb{R}_{\leqslant 0}$ maps $[\mathbb{R}] \in \mathrm{K}_{1}\left(\mathrm{C}^{*} \mathrm{~L}(\mathbb{R})\right)$ to the unit element $1 \in \mathrm{K}_{0}(\mathbf{C}) \cong \mathrm{K}_{0}\left(\mathrm{C}^{*} \mathrm{~L}(\{0\})\right)$. 
Proof. In our setup, we identify $[\mathbb{R}] \in \mathrm{K}_{1}\left(\mathrm{C}^{*} \mathrm{~L}(\mathbb{R})\right)$ with $\left[\alpha_{\mathbb{R}}\right] \times b \in \mathrm{K}_{0}\left(\mathrm{C}^{*} \mathrm{~L}(\mathbb{R}) \otimes \mathcal{C}_{0}(\mathbb{R})\right)$, where $b=b_{1} \in \mathrm{K}_{0}\left(\mathcal{C}_{0}(\mathbb{R}) \widehat{\otimes} \mathrm{Cl}_{1}^{*}\right)$ is the dual Dirac element, see Subsection 1.1.3, and $\alpha_{\mathbb{R}}: \mathcal{S} \rightarrow \mathrm{C}^{*} \mathrm{~L}(\mathbb{R}) \widehat{\otimes} \mathrm{Cl}_{1}$ is defined as in Subsection 2.2.1. The following diagram commutes:

$$
\begin{aligned}
\mathrm{K}_{*}\left(\mathcal{C}_{0}(\mathbb{R}) \widehat{\otimes} \mathrm{Cl}_{1}^{*}\right) \stackrel{\left[\alpha_{\mathbb{R}}\right] \times_{-}}{\longrightarrow} \mathrm{K}_{*} & \left(\mathrm{C}^{*} \mathrm{~L}(\mathbb{R}) \otimes \mathcal{C}_{0}(\mathbb{R})\right) \\
\mathrm{K}_{*}(\mathbf{C}) . & \longleftarrow \alpha_{*}
\end{aligned}
$$

Here $\alpha: \mathcal{S} \widehat{\otimes} \mathcal{C}_{0}(\mathbb{R}) \rightarrow \mathbb{K} \widehat{\otimes} \mathrm{Cl}_{1}$ and $\gamma: \mathrm{C}^{*} \mathrm{~L}(\mathbb{R}) \otimes \mathcal{C}_{0}(\mathbb{R}) \rightarrow \mathbb{K}$ are the asymptotic morphisms mentioned in Subsection 1.1.3 and Remark 2.2.4. In particular, we obtain $\gamma_{*}([\mathbb{R}])=\alpha_{*}(b)=1$.

The Mayer-Vietoris boundary map is induced by the inclusion

$$
\mathrm{C}^{*} \mathrm{~L}(\mathbb{R}) \otimes \mathcal{C}_{0}(\mathbb{R})=\mathcal{C}_{0}\left(\mathbb{R}, \mathrm{C}^{*} \mathrm{~L}(\mathbb{R})\right) \hookrightarrow \Omega\left(\mathrm{C}^{*} \mathrm{~L}(\mathbb{R}) ; \mathrm{C}^{*} \mathrm{~L}\left(\mathbb{R}_{\geqslant 0} \subset \mathbb{R}\right), \mathrm{C}^{*} \mathrm{~L}\left(\mathbb{R}_{\leqslant 0} \subset \mathbb{R}\right)\right) .
$$

We will use the symbol $\Omega$ as a shorthand to denote the latter $\mathrm{C}^{*}$-algebra. It is equal to the following sum of ideals inside $\mathrm{C}^{*} \mathrm{~L}(\mathbb{R}) \otimes \mathcal{C}([-\infty, \infty])$ :

$$
\Omega=\mathrm{C}^{*} \mathrm{~L}\left(\mathbb{R}_{\geqslant 0} \subset \mathbb{R}\right) \otimes \mathcal{C}_{0}([-\infty, \infty))+\mathrm{C}^{*} \mathrm{~L}\left(\mathbb{R}_{\leqslant 0} \subset \mathbb{R}\right) \otimes \mathcal{C}_{0}((-\infty, \infty]) .
$$

Moreover, $\gamma$ extends to an asymptotic morphism

$$
\bar{\gamma}: \mathrm{C}^{*} \mathrm{~L}(\mathbb{R}) \otimes \mathcal{C}([-\infty, \infty]) \rightarrow \mathbb{B}\left(\mathrm{L}^{2}(\mathbb{R})\right), \quad \bar{\gamma}_{t}(L \otimes f)=L(t) f,
$$

which, by (2.3.8), restricts to an asymptotic morphism $\tilde{\gamma}: \Omega \rightarrow \mathbb{K}\left(\mathrm{L}^{2}(\mathbb{R})\right)$. We obtain the following commutative diagram of (asymptotic) morphisms:

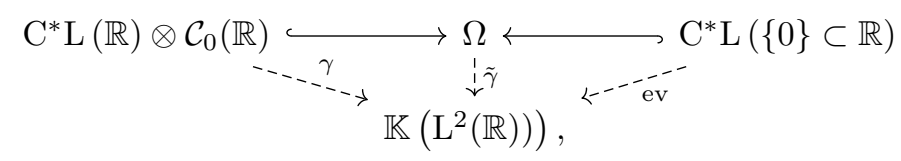

All asymptotic morphisms above induce isomorphisms on K-theory. By definition, the Mayer-Vietoris boundary map is the map $\partial_{\mathrm{MV}}: \mathrm{K}_{1}\left(\mathrm{C}^{*} \mathrm{~L}(\mathbb{R})\right)=\mathrm{K}_{0}\left(\mathrm{C}^{*} \mathrm{~L}(\mathbb{R}) \otimes \mathcal{C}_{0}(\mathbb{R})\right) \rightarrow$ $\mathrm{K}_{0}\left(\mathrm{C}^{*} \mathrm{~L}(\{0\} \subset \mathbb{R})\right) \cong \mathrm{K}_{0}\left(\mathrm{C}^{*} \mathrm{~L}(\{0\})\right) \cong \mathrm{K}_{0}(\mathbf{C})$ induced by the upper row of the diagram composed with ev. This finishes the proof since we already know that $\gamma_{*}([\mathbb{R}])=1$.

Theorem 2.3.9 (Suspension isomorphism). Let $X$ be a proper metric space endowed with a free and proper $\Gamma$-action and $Z \subseteq X$ some closed $\Gamma$-invariant subset. Then the map

$$
\mathrm{S}_{*}^{\Gamma}(X / / Z) \rightarrow \mathrm{S}_{*+1}^{\Gamma}(X \times \mathbb{R} / / Z \times \mathbb{R}), \quad x \mapsto x \otimes[\mathbb{R}],
$$

is an isomorphism. Its inverse is given by the Mayer-Vietoris boundary map

$$
\partial_{\mathrm{MV}}: \mathrm{S}_{*+1}^{\Gamma}(X \times \mathbb{R} / / Z \times \mathbb{R}) \rightarrow \mathrm{S}_{*}^{\Gamma}(X / / Z)
$$

associated to the cover $X \times \mathbb{R}=X \times \mathbb{R}_{\geqslant 0} \cup X \times \mathbb{R}_{\leqslant 0}$.

If $X$ is a complete spin manifold and $g \in \mathcal{R}_{Z}^{+}(X)^{\Gamma}$, then

$$
\left.\partial_{\mathrm{MV}}\left(\rho_{Z \times \mathbb{R}}^{\Gamma}\left(g \oplus \mathrm{d} t^{2}\right)\right)\right)=\rho_{Z}^{\Gamma}(g) .
$$


Proof. The Mayer-Vietoris boundary map $\partial_{\mathrm{MV}}$ is an isomorphism by Proposition 2.1.15. Therefore, it suffices for the first statement to show that $\partial_{\mathrm{MV}}(x \otimes[\mathbb{R}])=x$ for all $x \in \mathrm{K}_{n}\left(\mathrm{C}_{\Gamma}^{*} \mathrm{~L}_{Z}(X)\right)$. Let $W:=X \times \mathbb{R}$. From the discussion of Mayer-Vietoris sequences in Subsection 1.1.4, we obtain a commutative diagram as follows:

$$
\begin{aligned}
& \left.\mathrm{K}_{n}\left(\mathrm{C}_{\Gamma}^{*} \mathrm{~L}_{Z}(X)\right) \otimes \mathrm{K}_{1}\left(\mathrm{C}^{*} \mathrm{~L}(\mathbb{R})\right) \underset{\text { id } \otimes \partial_{\mathrm{MV}}}{\longrightarrow} \mathrm{K}_{n}\left(\mathrm{C}_{\Gamma}^{*} \mathrm{~L}_{Z}(X)\right) \otimes \mathrm{K}_{0}\left(\mathrm{C}^{*} \mathrm{~L}(\{0\} \subset \mathbb{R})\right)\right) \longleftarrow \mathrm{K}_{n}\left(\mathrm{C}_{\Gamma}^{*} \mathrm{~L}_{Z}(X)\right) \otimes \mathrm{K}_{0}\left(\mathrm{C}^{*} \mathrm{~L}(\{0\})\right) \\
& \mathrm{K}_{n+1}\left(\mathrm{C}_{\Gamma}^{*} \mathrm{~L}_{Z}(X) \widehat{\otimes} \mathrm{C}^{*} \mathrm{~L}(\mathbb{R})\right) \stackrel{\partial_{\mathrm{MV}}}{\longrightarrow} \mathrm{K}_{n}\left(\mathrm{C}_{\Gamma}^{*} \mathrm{~L}_{Z}(X) \widehat{\otimes} \mathrm{C}^{*} \mathrm{~L}(\{0\} \subset \mathbb{R})\right) \longleftarrow \mathrm{K}_{n}\left(\mathrm{C}_{\Gamma}^{*} \mathrm{~L}_{Z}(X) \widehat{\downarrow} \widehat{\otimes} \mathrm{C}^{*} \mathrm{~L}(\{0\})\right) \\
& \mathrm{K}_{n+1}\left(\mathrm{C}_{\Gamma}^{*} \mathrm{~L}_{Z \times \mathbb{R}}(W)\right) \stackrel{\partial_{\mathrm{MV}}}{\longrightarrow} \mathrm{K}_{n}\left(\mathrm{C}_{\Gamma}^{*} \mathrm{~L}_{Z \times\{0\}} \stackrel{\downarrow \mathrm{t}_{*}}{ }(X \times\{0\} \subset W)\right) \longleftarrow \stackrel{\downarrow \mathrm{t}_{*}}{\cong} \mathrm{K}_{n}\left(\mathrm{C}_{\Gamma}^{*} \mathrm{~L}_{Z}(X)\right)
\end{aligned}
$$

In view of this diagram, it is enough that $\partial_{\mathrm{MV}}([\mathbb{R}]) \in \mathrm{K}_{0}\left(\mathrm{C}^{*} \mathrm{~L}(\{0\} \subset \mathbb{R})\right)$ agrees with the unit element $1 \in \mathrm{K}_{0}(\mathbf{C}) \cong \mathrm{K}_{0}\left(\mathrm{C}^{*} \mathrm{~L}(\{0\})\right) \cong \mathrm{K}_{0}\left(\mathrm{C}^{*} \mathrm{~L}(\{0\} \subset \mathbb{R})\right)$, which is precisely the content of Lemma 2.3.8. Thus $\partial_{\mathrm{MV}}$ and taking the external product with $[\mathbb{R}]$ are mutually inverse isomorphisms.

Theorem 2.3.1 implies $\rho_{Z \times \mathbb{R}}^{\Gamma}\left(g \oplus \mathrm{d} t^{2}\right)=\rho_{Z}^{\Gamma}(g) \otimes[\mathbb{R}]$. Hence the second statement is a direct consequence of the first.

There are analogous results concerning the APS-index and index difference:

Proposition 2.3.10. Let $X$ be a proper metric space endowed with a proper and free $\Gamma$-action and $Z \subseteq X$ some closed $\Gamma$-invariant subset. Then the map

$$
\mathrm{K}_{*}\left(\frac{\mathrm{C}_{\Gamma}^{*}(X)}{\mathrm{C}_{\Gamma}^{*}(Z \subset X)}\right) \rightarrow \mathrm{K}_{*+1}\left(\frac{\mathrm{C}_{\Gamma}^{*}(X \times \mathbb{R})}{\mathrm{C}_{\Gamma}^{*}(Z \times \mathbb{R} \subset X \times \mathbb{R})}\right), \quad x \mapsto x \otimes \operatorname{Ind}(\mathbb{R}),
$$

is an isomorphism. Its inverse is given by the Mayer-Vietoris boundary map from Proposition 2.1.11,

$$
\partial_{\mathrm{MV}}: \mathrm{K}_{*+1}\left(\frac{\mathrm{C}_{\Gamma}^{*}(X \times \mathbb{R})}{\mathrm{C}_{\Gamma}^{*}(Z \times \mathbb{R} \subset X \times \mathbb{R})}\right) \rightarrow \mathrm{K}_{*}\left(\frac{\mathrm{C}_{\Gamma}^{*}(X)}{\mathrm{C}_{\Gamma}^{*}(Z \subset X)}\right)
$$

associated to the cover $X \times \mathbb{R}=X \times \mathbb{R}_{\geqslant 0} \cup X \times \mathbb{R}_{\leqslant 0}$.

Proof. By Corollary 2.1.14, $\mathrm{K}_{*}\left(\mathrm{C}_{\Gamma}^{*}\left(X \times \mathbb{R}_{\geqslant 0}\right)\right)$ and $\mathrm{K}_{*}\left(\mathrm{C}_{\Gamma}^{*}\left(Z \times \mathbb{R}_{\geqslant 0} \subset X \times \mathbb{R}_{\geqslant 0}\right)\right)$ vanish and hence $\mathrm{K}_{*}\left(\frac{\mathrm{C}_{\Gamma}^{*}\left(X \times \mathbb{R}_{\geqslant 0}\right)}{\mathrm{C}_{\Gamma}^{*}\left(Z \times \mathbb{R}_{\geqslant 0} \subset X \times \mathbb{R}\right)}\right)$ vanishes, and similarly for $X \times \mathbb{R}_{\leqslant 0}$. This implies that $\partial_{\mathrm{MV}}$ is an isomorphism. Hence it suffices to observe that $\partial_{\mathrm{MV}}(x \otimes \operatorname{Ind}(\mathbb{R}))=x$. Indeed, as in the proof of Theorem 2.3.9, we obtain:

$$
\partial_{\mathrm{MV}}(x \otimes \operatorname{Ind}(\mathbb{R}))=x \otimes \partial_{\mathrm{MV}}(\operatorname{Ind}(\mathbb{R}))=x,
$$

where the latter is the Mayer-Vietoris boundary map associated to $\mathbb{R}=\mathbb{R}_{\geqslant 0} \cup \mathbb{R}_{\leqslant 0}$ and we use that $\partial_{\mathrm{MV}}(\operatorname{Ind}(\mathbb{R}))=\operatorname{Ind}\left(\partial_{\mathrm{MV}}([\mathbb{R}])\right)=1 \in \mathrm{K}_{0}\left(\mathrm{C}^{*}(\{0\})\right) \cong \mathrm{K}_{0}(\mathbf{C})$.

Corollary 2.3.11 (see Corollary 2.3.6). Let $Y$ be a complete spin manifold endowed with a free and proper $\Gamma$-action and with boundary $\partial Y=X$. Let $Z \subseteq X$ be a $\Gamma$-invariant subset and $g \in \mathcal{R}_{Z}^{+}(X)^{\Gamma}$. Then

$$
\partial_{\mathrm{MV}}\left(\operatorname{Ind}_{\mathrm{APS}}^{\Gamma}\left(Y \times \mathbb{R}, X \times \mathbb{R} / / Z \times \mathbb{R}, g \oplus \mathrm{d} t^{2}\right)\right)=\operatorname{Ind}_{\mathrm{APS}}^{\Gamma}(Y, X / / Z, g) .
$$


Corollary 2.3.12 (see Corollary 2.3.7). Let $X$ be a complete spin manifold endowed a free and proper $\Gamma$-action. Let $Z \subseteq X$ be a $\Gamma$-invariant subset and $g_{0}, g_{1} \in \mathcal{R}_{Z}^{+}(X)$. Then:

$$
\partial_{\mathrm{MV}}\left(\operatorname{Ind}_{\mathrm{diff}}^{\Gamma}\left(g_{0} \oplus \mathrm{d} t^{2}, g_{1} \oplus \mathrm{d} t^{2} / / Z \times \mathbb{R}\right)\right)=\operatorname{Ind}_{\mathrm{diff}}^{\Gamma}\left(g_{0}, g_{1} / / Z\right) .
$$

\subsubsection{Hypereuclidean manifolds}

By the results in the previous subsection, secondary invariants can still distinguish metrics of positive scalar curvature after taking products with the real line, and by iteration, even after taking products with Euclidean space of any dimension. We can push this idea a little bit further by considering the following concept, which is originally due to Gromov [Gro93].

Definition 2.3.13. A complete Riemannian manifold $Y$ is called hypereuclidean if it admits a proper Lipschitz map $Y \rightarrow \mathbb{R}^{q}$ of degree 1 into some Euclidean space $\mathbb{R}^{q}$ (if this is the case, then of course $q=\operatorname{dim} Y$ ). Furthermore, we say that $Y$ is stably hypereuclidean if $Y \times \mathbb{R}^{k}$ is hypereuclidean for some $k \geqslant 0$.

The following result of Dranishnikov provides many examples of stably hypereuclidean manifolds:

Theorem 2.3.14 ([Dra06]). Let $B$ be a closed aspherical manifold and suppose that $\pi_{1}(B)$ has finite asymptotic dimension. Then the universal covering $\tilde{B}$ is stably hypereuclidean.

Products with stably hypereuclidean manifolds are split-injective on the partial structure groups:

Proposition 2.3.15. Let $X$ be a proper metric space endowed with a proper and free $\Gamma$-action, $Z \subset X$ a $\Gamma$-invariant subset. Suppose that $Y$ is a q-dimensional complete spin manifold that is stably hypereuclidean and endowed with a $\Lambda$-action. Then the map

$$
\mathrm{S}_{*}^{\Gamma}(X / / Z) \rightarrow \mathrm{S}_{*+q}^{\Gamma \times \Lambda}(X \times Y / / Z \times Y), \quad x \mapsto x \otimes[Y]^{\Gamma},
$$

is split-injective. Moreover, the retraction can be chosen to be natural in X.

Proof. It is enough to give a proof for the case that $Y$ is hypereuclidean and $\Lambda$ is the trivial group. This is because for each $k \geqslant 0$ we have a canonical map

$$
\mathrm{S}_{*+q}^{\Gamma \times \Lambda}(X \times Y / / Z \times Y) \rightarrow \mathrm{S}_{*+q+k}^{\Gamma}\left(X \times Y \times \mathbb{R}^{k} / / Z \times Y \times \mathbb{R}^{k}\right),
$$

which forgets $\Lambda$-equivariance and takes the external product with $\left[\mathbb{R}^{k}\right]$.

Now suppose that $f: Y \rightarrow \mathbb{R}^{q}$ is a proper Lipschitz map of degree 1 . Then the induced map on K-homology takes the fundamental class of $Y$ to the fundamental class of $\mathbb{R}^{q}$. Thus the map $\left(\operatorname{id}_{X} \times f\right)_{*}: \mathrm{S}_{*+q}^{\Gamma}(X \times Y / / Z \times Y) \rightarrow \mathrm{S}_{*+q}^{\Gamma}\left(X \times \mathbb{R}^{q} / / Z \times \mathbb{R}^{q}\right)$ takes elements of the form $x \otimes[Y]$ to $x \otimes\left[\mathbb{R}^{q}\right]$. So, it even suffices to prove the claim for $Y=\mathbb{R}^{q}$. However, for $Y=\mathbb{R}^{q}$, the result follows from (an iterated application of) Theorem 2.3.9. 
An analogous argument yields:

Proposition 2.3.16. Let $X$ be a proper metric space endowed with a proper and free $\Gamma$-action, $Z \subset X$ a $\Gamma$-invariant subset. Suppose that $Y$ is a q-dimensional complete spin manifold that is stably hypereuclidean and endowed with a $\Lambda$-action. Then the map

$$
\mathrm{K}_{*}\left(\frac{\mathrm{C}_{\Gamma}^{*}(X)}{\mathrm{C}_{\Gamma}^{*}(Z \subset X)}\right) \rightarrow \mathrm{K}_{*+q}\left(\frac{\mathrm{C}_{\Gamma}^{*}(X \times Y)}{\mathrm{C}_{\Gamma}^{*}(Z \times Y \subset X \times Y)}\right), \quad x \mapsto x \otimes \operatorname{Ind}^{\Lambda}(Y),
$$

is split-injective. Moreover, the retraction can be chosen to be natural in $X$.

\subsection{Secondary index theorems}

\subsubsection{Partitioned manifold index theorems}

In this subsection, we establish a new partitioned manifold index theorem for partial secondary invariants, which generalizes the secondary partitioned manifold index theorem [PS14, Theorem 1.22].

Definition 2.4.1. Let $W$ be a complete spin manifold. Let $X \subset W$ be a submanifold of codimension one with trivial normal bundle and a fixed tubular neighborhood $c: X \times[-1,1] \hookrightarrow W$. We suppose that $W \backslash X$ has two connected components and denote the closures of the connected components of $W \backslash X$ by $W_{-}$and $W_{+}$so that $c(X \times\{ \pm 1\}) \subseteq W_{ \pm}$. Then $W_{ \pm}$are submanifolds of $W$ with common boundary $\partial W_{ \pm}=X$. We require that the cover of $W$ by $W_{+}$and $W_{-}$is uniformly excisive with respect to the implicitly fixed proper metric $d_{W}$. If $W$ is endowed with a $\Gamma$-action, we additionally require that $X, W_{-}$and $W_{+}$are all $\Gamma$-invariant subsets.

In this situation, we say that $W$ is partitioned by $X$. Moreover, we say that a Riemannian metric $h \in \mathcal{R}(W)$ is partitioned by $g \in \mathcal{R}(X)$ if $c^{*} h \uparrow X \times(-\varepsilon, \varepsilon)=g \oplus \mathrm{d} t^{2}$ for some $\varepsilon>0$.

Definition 2.4.2. Let $W$ be partitioned by $X$. Let $Z \subset W$ be a closed subset. We say $Z$ is admissible with respect to $W_{+}$if

(i) the subset $Z$ is of product structure near $X$ with respect to the fixed tubular neighborhood, that is, $c^{-1}(Z) \cap(X \times(-\varepsilon, \varepsilon))=(Z \cap X) \times(-\varepsilon, \varepsilon)$ for some $\varepsilon>0$.

(ii) for every $R>0$ there exists $S>0$ such that

$$
\mathcal{U}_{R}\left(Z \cap W_{+}\right) \cap \mathcal{U}_{R}(X) \subseteq \mathcal{U}_{S}(Z \cap X)
$$

Example 2.4.3. If $W_{+}=X \times[0, \infty)$ and $Z \cap W_{+}=Z \cap X \times[0, \infty)$, then $Z$ is admissible with respect to $W_{+}$.

Remark 2.4.4. It follows from Lemma 2.1.10, that if $Z$ is admissible with respect to $W_{+}$, then for all $R>0$ there exists $S>0$ such that

$$
\begin{gathered}
\mathcal{U}_{R}(Z) \cap \mathcal{U}_{R}\left(W_{-}\right) \subseteq \mathcal{U}_{S}\left(Z \cap W_{-}\right), \\
\mathcal{U}_{R}\left(Z \cap W_{-}\right) \cap \mathcal{U}_{R}\left(Z \cap W_{+}\right) \subseteq \mathcal{U}_{S}(Z \cap X) .
\end{gathered}
$$


Figure 2.1: Illustration of a subset admissible with respect to $W_{+}$but not $W_{-}$

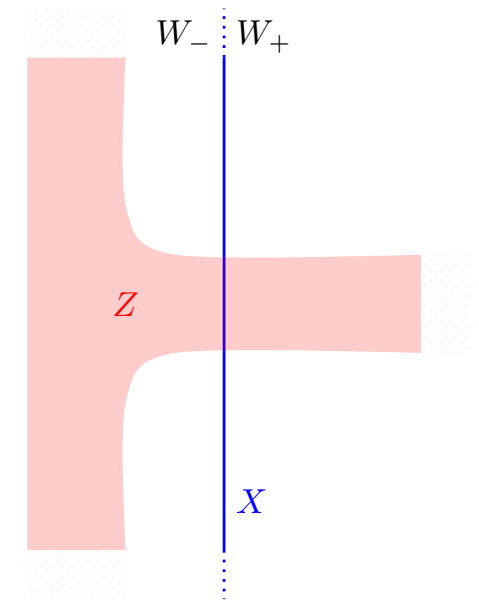

In fact, (2.4.3) says that the cover of $Z$ by $Z \cap W_{-}$and $Z \cap W_{+}$is also coarsely excisive.

Remark 2.4.5. As will become apparent in Lemmas 2.4.10 and 2.4.11, admissibility with respect to $W_{+}$is the condition that is needed to be able to replace the other half, $W_{-}$, by something else without changing the image of the partial $\rho$-invariant under the Mayer-Vietoris boundary map. This idea will be key to the proof of the main theorem below.

We have the following partitioned manifold index theorem for partial secondary invariants.

Theorem 2.4.6. Let $W$ be a complete spin manifold endowed with a free and proper $\Gamma$ action and suppose that it is partitioned by $X$. Let $Z \subseteq W$ be a closed $\Gamma$-invariant subset that is admissible with respect to $W_{+}$and suppose that $h \in \mathcal{R}_{Z}^{+}(W)^{\Gamma}$ is partitioned by $g \in \mathcal{R}_{Z \cap X}^{+}(X)^{\Gamma}$. Then the Mayer-Vietoris boundary map

$$
\partial_{\mathrm{MV}}: \mathrm{S}_{*+1}^{\Gamma}(W / / Z) \rightarrow \mathrm{S}_{*}^{\Gamma}(X / / Z \cap X)
$$

associated to the cover $W=W_{+} \cup W_{-}$satisfies

$$
\partial_{\mathrm{MV}}\left(\rho_{Z}^{\Gamma}(h)\right)=\rho_{Z \cap X}^{\Gamma}(g) \text {. }
$$

Remark 2.4.7. There is an apparent asymmetry between $W_{+}$and $W_{-}$due to the admissibility condition. This is resolved by observing that the proof of the partitioned manifold index theorem also works with the roles of $W_{-}$and $W_{+}$exchanged; so the theorem also holds if the subset is admissible with respect to $W_{-}$but not with respect to $W_{+}$. For the proof of the delocalized APS index theorem in Subsection 2.4.2 it will be crucial that we do not need simultaneous admissibility with respect to both halves of the partition. 
Before turning to the proof of Theorem 2.4.6, we discuss a few consequences. Firstly, taking $Z=\emptyset$, this recovers the secondary partitioned manifold index theorem of PiazzaSchick [PS14, Theorem 1.22] in all dimensions. Moreover, we obtain a partitioned manifold index theorem for the coarse APS-index as in the corollary below.

Corollary 2.4.8. Let $V$ be a complete Riemannian spin manifold endowed with a free and proper $\Gamma$-action and with boundary $\partial V=W$. Suppose that $V$ is partitioned by a manifold $Y$ with boundary $\partial Y=X$ such that $X \subseteq W$ and $W$ is partitioned by $X$. Let $Z \subseteq W$ be a closed $\Gamma$-invariant subset that is admissible with respect to both $W_{+}$and $W_{-}$. Let $h \in \mathcal{R}_{Z}^{+}(W)^{\Gamma}$ be partitioned by $g \in \mathcal{R}_{Z \cap X}^{+}(X)^{\Gamma}$. Then the Mayer-Vietoris boundary map

$$
\partial_{\mathrm{MV}}: \mathrm{K}_{n+2}\left(\frac{\mathrm{C}_{\Gamma}^{*}(W)}{\mathrm{C}_{\Gamma}^{*}(Z \subset W)}\right) \rightarrow \mathrm{K}_{n+1}\left(\frac{\mathrm{C}_{\Gamma}^{*}(X)}{\mathrm{C}_{\Gamma}^{*}(Z \cap X \subset X)}\right)
$$

associated to the cover $W=W_{+} \cup W_{-}$satisfies

$$
\partial_{\mathrm{MV}}\left(\operatorname{Ind}_{\mathrm{APS}}^{\Gamma}(V, W / / Z, h)\right)=\operatorname{Ind}_{\mathrm{APS}}^{\Gamma}(Y, X / / Z \cap X, g) .
$$

Proof. Granted Theorem 2.4.6, this is a straightforward generalization of Corollary 2.3.11. The fact that $Z$ is admissible with respect to both $W_{+}$and $W_{-}$is equivalent to the condition in Proposition 2.1.11; so the Mayer-Vietoris boundary map mentioned in the statement exists. Let $V_{\infty}:=V \cup_{\partial V}\left(W \times \mathbb{R}_{\geqslant 0}\right)$ and $Y_{\infty}:=Y \cup_{\partial Y}\left(X \times \mathbb{R}_{\geqslant 0}\right)$ as in the definition of the coarse APS-index, see Subsection 2.2.3. Let $h_{V} \in \mathcal{R}_{Z}^{+}(V)$ such that $\partial h_{V}=h$. We may assume that $h_{V}$ is partitioned by $h_{Y} \in \mathcal{R}_{Z \cap Y}^{+}(X)$ with $\partial h_{Y}=g$. Then $\left(h_{V}\right)_{\infty} \in \mathcal{R}_{Z_{\infty}}^{+}\left(V_{\infty}\right)^{\Gamma}$ is partitioned by $\left(h_{Y}\right)_{\infty} \in \mathcal{R}_{(Z \cap Y)_{\infty}}^{+}\left(Y_{\infty}\right)^{\Gamma}$ and it follows from Theorem 2.4.6 that

$$
\partial_{\mathrm{MV}}\left(\operatorname{Ind}_{Z_{\infty}}^{\Gamma}\left(V_{\infty},\left(h_{V}\right)_{\infty}\right)\right)=\operatorname{Ind}_{Z_{\infty} \cap Y_{\infty}}^{\Gamma_{\infty}}\left(Y_{\infty},\left(h_{Y}\right)_{\infty}\right)
$$

where $\partial_{\mathrm{MV}}: \mathrm{K}_{n+2}\left(\mathrm{C}_{\Gamma}^{*}\left(Z_{\infty} \subset V_{\infty}\right)\right) \rightarrow \mathrm{K}_{n+1}\left(\mathrm{C}_{\Gamma}^{*}\left(Z_{\infty} \cap Y_{\infty} \subset Y_{\infty}\right)\right)$. As in the proof of Corollary 2.3.11, this suffices to prove the statement by the definition of the coarse APS-index.

Corollary 2.4.9. Let $W$ be a complete spin manifold endowed with a free and proper $\Gamma$-action which is partitioned by $X \subset W$. Let $Z \subseteq W$ be a closed $\Gamma$-invariant subset that is admissible both with respect to $W_{+}$and $W_{-}$. For $i=0,1$, let $h_{i} \in \mathcal{R}_{Z}^{+}(W)^{\Gamma}$ be partitioned by $g_{i} \in \mathcal{R}_{Z \cap X}^{+}(X)^{\Gamma}$. Then the Mayer-Vietoris boundary map

$$
\partial_{\mathrm{MV}}: \mathrm{K}_{n+2}\left(\frac{\mathrm{C}_{\Gamma}^{*}(W)}{\mathrm{C}_{\Gamma}^{*}(Z \subset W)}\right) \rightarrow \mathrm{K}_{n+1}\left(\frac{\mathrm{C}_{\Gamma}^{*}(X)}{\mathrm{C}_{\Gamma}^{*}(Z \cap X \subset X)}\right)
$$

associated to the cover $W=W_{+} \cup W_{-}$satisfies

$$
\partial_{\mathrm{MV}}\left(\operatorname{Ind}_{\mathrm{diff}}^{\Gamma}\left(h_{0}, h_{1} / / Z\right)\right)=\operatorname{Ind}_{\mathrm{diff}}^{\Gamma}\left(g_{0}, g_{1} / / Z \cap X\right) .
$$


Furthermore, Theorem 2.4.6 implies the concordance invariance of partial secondary invariants which is still open from Subsection 2.2.2.

Proof of Proposition 2.2.11. Let $g_{0}, g_{1} \in \mathcal{R}_{Z}^{+}(X)^{\Gamma}$ be concordant relative to $Z$ as in Definition 1.3.5. Let $h \in \mathcal{R}_{Z}^{+}(W)$ with $W:=X \times \mathbb{R}$ such that the conditions of Definition 1.3 .5 are satisfied. We identify $X$ with (say) $X \times\{-1\} \subseteq W$ and view $(W, h)$ as being partitioned by $\left(X, g_{0}\right)$ with $W_{-}=(-\infty,-1] \times X$ and $W_{+}=[-1, \infty) \times X$. Thus by Theorem 2.4.6 implies

$$
\partial_{\mathrm{MV}} \rho_{Z \times \mathbb{R}}^{\Gamma}(h)=\rho_{Z}^{\Gamma}\left(g_{0}\right) .
$$

Then Theorem 2.3.9 implies

$$
\rho_{Z \times \mathbb{R}}^{\Gamma}(h)=\rho_{Z}^{\Gamma}\left(g_{0}\right) \otimes[\mathbb{R}] .
$$

However, we can also identify $X$ with $X \times\{2\} \subset W$ and apply the same argument again to show that

$$
\rho_{Z \times \mathbb{R}}^{\Gamma}(h)=\rho_{Z}^{\Gamma}\left(g_{1}\right) \otimes[\mathbb{R}] .
$$

By Theorem 2.3.9, these two facts imply that $\rho_{Z}^{\Gamma}\left(g_{0}\right)=\rho_{Z}^{\Gamma}\left(g_{1}\right)$.

We now proceed with technical lemmas needed for the proof of Theorem 2.4.6. If $W$ is partitioned by $X$, then a $\left(W, \Gamma, \mathrm{Cl}_{n+1}\right)$-module $\mathfrak{H}$ can be restricted to a $\left(W_{+}, \Gamma, \mathrm{Cl}_{n+1}\right)$ module $\mathfrak{H}_{+}:=\operatorname{ran}\left(\mathbf{1}_{W_{+}}\right)$using the projection in $\mathfrak{H}$ corresponding to the characteristic function of $W_{+}$. This will be used implicitly in the following.

Lemma 2.4.10. Let $Z \subseteq W$ be a closed $\Gamma$-invariant subset that is admissible with respect to $W_{+}$. Then for all $p \in \mathbb{N}$ :

(i) The inclusion $\mathfrak{H}_{+} \subset \mathfrak{H}$ induces $*$-isomorphisms:

$$
\begin{aligned}
& \frac{\mathrm{C}_{\Gamma}^{*} \mathrm{~L}_{Z \cap W_{+}}\left(W_{+} ; \mathrm{Cl}_{p}\right)}{\mathrm{C}_{\Gamma}^{*} \mathrm{~L}_{Z \cap X}\left(X \subset W_{+} ; \mathrm{Cl}_{p}\right)} \stackrel{i}{\cong} \frac{\mathrm{C}_{\Gamma}^{*} \mathrm{~L}_{Z \cap W_{+}}\left(W_{+} \subset W ; \mathrm{Cl}_{p}\right)}{\mathrm{C}_{\Gamma}^{*} \mathrm{~L}_{Z \cap X}\left(X \subset W ; \mathrm{Cl}_{p}\right)} \\
& \stackrel{j}{\cong} \frac{\mathrm{C}_{\Gamma}^{*} \mathrm{~L}_{Z}\left(W ; \mathrm{Cl}_{p}\right)}{\mathrm{C}_{\Gamma}^{*} \mathrm{~L}_{Z \cap W_{-}}\left(W_{-} \subset W ; \mathrm{Cl}_{p}\right)} .
\end{aligned}
$$

If $Z$ is also admissible with respect to $W_{-}$, then the inverse of these isomorphisms is induced by the expectation

$$
\Phi: \mathrm{C}_{\Gamma}^{*}\left(W ; \mathrm{Cl}_{p}\right) \rightarrow \mathrm{C}_{\Gamma}^{*}\left(W_{+} ; \mathrm{Cl}_{p}\right), \quad T \mapsto \mathbf{1}_{W_{+}} T \mathbf{1}_{W_{+}} .
$$

(ii) The following *-homomorphism is injective:

$$
\frac{\mathrm{C}_{\Gamma}^{*} \mathrm{~L}_{Z \cap W_{+}}\left(W_{+} ; \mathrm{Cl}_{p}\right)}{\mathrm{C}_{\Gamma}^{*} \mathrm{~L}_{Z \cap X}\left(X \subset W_{+} ; \mathrm{Cl}_{p}\right)} \hookrightarrow \frac{\mathrm{C}_{\Gamma}^{*} \mathrm{~L}\left(W_{+} ; \mathrm{Cl}_{p}\right)}{\mathrm{C}_{\Gamma}^{*} \mathrm{~L}\left(X \subset W_{+} ; \mathrm{Cl}_{p}\right)} .
$$


Proof. To simplify the exposition, we drop the Clifford algebra and the group $\Gamma$ from the notation during this proof.

The map $j$ in (i) is an isomorphism by Lemma 2.1.7. To show that the map $i$ in (i) is also an isomorphism, we need,

$$
\begin{aligned}
\mathrm{C}^{*}\left(Z \cap W_{+} \subset W_{+}\right) \cap \mathrm{C}^{*}(Z \cap X \subset W) & =\mathrm{C}^{*}\left(Z \cap X \subset W_{+}\right), \\
\mathrm{C}^{*} \mathrm{~L}\left(W_{+}\right) \cap \mathrm{C}^{*} \mathrm{~L}(X \subset W) & =\mathrm{C}^{*} \mathrm{~L}\left(X \subset W_{+}\right), \\
\mathrm{C}^{*} \mathrm{~L}_{Z \cap W_{+}}\left(W_{+}\right)+\mathrm{C}^{*} \mathrm{~L}_{Z \cap X}(X \subset W) & =\mathrm{C}^{*} \mathrm{~L}_{Z \cap W_{+}}\left(W_{+} \subset W\right) .
\end{aligned}
$$

Indeed, consider the expectation $\Phi: \mathrm{C}^{*}(W) \rightarrow \mathrm{C}^{*}\left(W_{+}\right), T \mapsto \mathbf{1}_{W_{+}} T \mathbf{1}_{W_{+}}$. Note that $\operatorname{supp}(\Phi(T)) \subseteq \operatorname{supp}(T) \cap\left(W_{+} \times W_{+}\right)$for all $T \in \mathrm{C}^{*}(W)$. In particular, $\Phi$ extends to an expectation $\mathrm{C}^{*} \mathrm{~L}(W) \rightarrow \mathrm{C}^{*} \mathrm{~L}\left(W_{+}\right)$, which we also denote by $\Phi$. We have $\Phi\left(\mathrm{C}^{*}(Z \cap X \subset W)\right)=\mathrm{C}^{*}\left(Z \cap X \subset W_{+}\right)$and $\Phi\left(\mathrm{C}^{*} \mathrm{~L}(X \subset W)\right)=\mathrm{C}^{*} \mathrm{~L}\left(X \subset W_{+}\right)$, which proves (2.4.4) and (2.4.5). To prove (2.4.6), let $L \in \mathrm{C}^{*} \mathrm{~L}_{Z \cap W_{+}}\left(W_{+} \subset W\right)$. Then $\Phi(L) \in \mathrm{C}^{*} \mathrm{~L}_{Z \cap W_{+}}\left(W_{+}\right)$and $L-\Phi(L) \in \mathrm{C}^{*} \mathrm{~L}(X \subset W)$. Moreover, we have $\operatorname{ev}_{1}(L)-$ $\Phi\left(\operatorname{ev}_{1}(L)\right) \in \mathrm{C}^{*}\left(Z \cap W_{+} \subset W\right) \cap \mathrm{C}^{*}(X \subset W)$. In addition, using the admissibility condition (2.4.1), we obtain (compare Lemma 2.1.6):

$$
\mathrm{C}^{*}\left(Z \cap W_{+} \subset W\right) \cap \mathrm{C}^{*}(X \subset W)=\mathrm{C}^{*}(Z \cap X \subset W) .
$$

Thus the decomposition $\Phi(L)+(L-\Phi(L))=L$ proves (2.4.6) and shows that the inverse of $i$ is always induced by $\Phi$ (even if $Z$ is not admissible with respect to $W_{-}$).

It does not hold in general that $\Phi\left(\mathrm{C}^{*} \mathrm{~L}_{Z \cap W_{-}}\left(W_{-} \subset W\right)\right) \subseteq \mathrm{C}^{*} \mathrm{~L}_{Z \cap X}(X \subset W)$ and $\Phi\left(\mathrm{C}^{*} \mathrm{~L}_{Z}(W)\right) \subseteq \mathrm{C}^{*} \mathrm{~L}_{Z \cap W_{+}}\left(W_{+} \subset W\right)$. However, if $Z$ is also admissible with respect to $W_{-}$, then these statements are in fact true and one can verify that $\Phi$ is inverse to $j$ (as follows from an argument using (2.4.1) to (2.4.3) with the roles of $W_{+}$and $W_{-}$ reversed).

Statement (ii) is a consequence of a version of (2.4.7) restricted to $W_{+}$, which also follows from (2.4.1).

The main ingredient to prove Theorem 2.4.6 is the following "swapping lemma", which implies that we can modify one half of a partitioned manifold without changing the image of the Mayer-Vietoris boundary map. Hence it reduces the general partitioned manifold index theorem to the product situation. This idea originated in a proof of Roe's partitioned manifold index theorem due to Higson [Hig91].

Lemma 2.4.11 (Compare [Hig91, Lemma 3.1]). Let $W$ and $\widetilde{W}$ be both partitioned by $X$. Suppose $\widetilde{W}_{+}=W_{+}$and that the restrictions of $d_{W}$ and $d_{\widetilde{W}}$ agree on $W_{+}$. Let $Z \subseteq W, \widetilde{Z} \subseteq \widetilde{W}$ be admissible with respect to $W_{+}=\widetilde{W}_{+}$with $Z \cap W_{+}=\widetilde{Z} \cap \widetilde{W}_{+}$. Let $h \in \mathcal{R}_{Z}^{+}(W)^{\Gamma}, \widetilde{h} \in \mathcal{R}_{\widetilde{Z}}^{+}(\widetilde{W})^{\Gamma}$ agree on $W_{+}$and be partitioned by some metric on $X$. Then

$$
\partial_{\mathrm{MV}}\left(\rho_{Z}^{\Gamma}(h)\right)=\partial_{\mathrm{MV}}\left(\rho_{\widetilde{Z}}^{\Gamma}(\widetilde{h})\right) \in \mathrm{S}_{n}^{\Gamma}(X / / Z \cap X) .
$$


Proof. Lemma 2.4 .10 (i) (applied to $Z=W$ ), implies isomorphisms

$$
\frac{\mathrm{C}_{\Gamma}^{*} \mathrm{~L}\left(W ; \mathrm{Cl}_{n+1}\right)}{\mathrm{C}_{\Gamma}^{*} \mathrm{~L}\left(W_{-} \subset W ; \mathrm{Cl}_{n+1}\right)} \stackrel{\Phi}{\rightarrow} \frac{\mathrm{C}_{\Gamma}^{*} \mathrm{~L}\left(W_{+} ; \mathrm{Cl}_{n+1}\right)}{\mathrm{C}_{\Gamma}^{*} \mathrm{~L}\left(X \subset W_{+} ; \mathrm{Cl}_{n+1}\right)} \stackrel{\widetilde{\Phi}}{\leftarrow} \frac{\mathrm{C}_{\Gamma}^{*} \mathrm{~L}\left(\widetilde{W} ; \mathrm{Cl}_{n+1}\right)}{\mathrm{C}_{\Gamma}^{*} \mathrm{~L}\left(\widetilde{W}-\subset \widetilde{W} ; \mathrm{Cl}_{n+1}\right)},
$$

where the maps $\Phi$ and $\widetilde{\Phi}$ are induced by $T \mapsto \mathbf{1}_{W_{+}} T \mathbf{1}_{W_{+}}$. Consider

$$
\begin{aligned}
& \alpha:=\alpha_{h}: \mathcal{S} \rightarrow \mathrm{C}_{\Gamma}^{*} \mathrm{~L}\left(W ; \mathrm{Cl}_{n+1}\right), \\
& \widetilde{\alpha}:=\alpha_{\tilde{h}}: \mathcal{S} \rightarrow \mathrm{C}_{\Gamma}^{*} \mathrm{~L}\left(\widetilde{W} ; \mathrm{Cl}_{n+1}\right),
\end{aligned}
$$

which have been defined in Subsection 2.2.1. Let

$$
\pi: \mathrm{C}_{\Gamma}^{*} \mathrm{~L}\left(W ; \mathrm{Cl}_{n+1}\right) \rightarrow \frac{\mathrm{C}_{\Gamma}^{*} \mathrm{~L}\left(W ; \mathrm{Cl}_{n+1}\right)}{\mathrm{C}_{\Gamma}^{*} \mathrm{~L}\left(W_{-} \subset W ; \mathrm{Cl}_{n+1}\right)}
$$

be the canonical quotient map and define $\widetilde{\pi}$ analogously. Some propagation speed estimates which we postpone until the end of this proof show that

$$
\mathbf{1}_{W_{+}} f\left(\frac{1}{\mathrm{t}} \mathscr{D}_{h}\right) \mathbf{1}_{W_{+}}-\mathbf{1}_{W_{+}} f\left(\frac{1}{\mathrm{t}} \mathfrak{D}_{\widetilde{h}}\right) \mathbf{1}_{W_{+}} \in \mathrm{C}_{\Gamma}^{*} \mathrm{~L}\left(X \subset W_{+} ; \mathrm{Cl}_{n+1}\right) \text {. }
$$

Thus the following $*$-homomorphisms are equal:

$$
\Phi \circ \pi \circ \alpha=\widetilde{\Phi} \circ \widetilde{\pi} \circ \widetilde{\alpha}: \mathcal{S} \rightarrow \frac{\mathrm{C}_{\Gamma}^{*} \mathrm{~L}\left(W_{+} ; \mathrm{Cl}_{n+1}\right)}{\mathrm{C}_{\Gamma}^{*} \mathrm{~L}\left(X \subset W_{+} ; \mathrm{Cl}_{n+1}\right)} .
$$

By Lemma 2.4.10 we also obtain isomorphisms,

$$
\begin{aligned}
\frac{\mathrm{C}_{\Gamma}^{*} \mathrm{~L}_{Z}\left(W ; \mathrm{Cl}_{n+1}\right)}{\mathrm{C}_{\Gamma}^{*} \mathrm{~L}_{Z \cap W_{-}}\left(W_{-} \subset W ; \mathrm{Cl}_{n+1}\right)} \stackrel{j}{\leftarrow} \frac{\mathrm{C}_{\Gamma}^{*} \mathrm{~L}_{Z \cap W_{+}}\left(W_{+} ; \mathrm{Cl}_{n+1}\right)}{\mathrm{C}_{\Gamma}^{*} \mathrm{~L}_{Z \cap X}\left(X \subset W_{+} ; \mathrm{Cl}_{n+1}\right)} \\
\stackrel{\tilde{\tilde{I}}}{\rightarrow} \frac{\mathrm{C}_{\Gamma}^{*} \mathrm{~L}_{\widetilde{Z}}\left(\widetilde{W} ; \mathrm{Cl}_{n+1}\right)}{\mathrm{C}_{\Gamma}^{*} \mathrm{~L}_{\widetilde{Z} \cap \widetilde{W}_{-}}\left(\widetilde{W}_{-} \subset \widetilde{W} ; \mathrm{Cl}_{n+1}\right)},
\end{aligned}
$$

and the map

$$
k: \frac{\mathrm{C}_{\Gamma}^{*} \mathrm{~L}_{Z \cap W_{+}}\left(W_{+} ; \mathrm{Cl}_{n+1}\right)}{\mathrm{C}_{\Gamma}^{*} \mathrm{~L}_{Z \cap X}\left(X \subset W_{+} ; \mathrm{Cl}_{n+1}\right)} \hookrightarrow \frac{\mathrm{C}_{\Gamma}^{*} \mathrm{~L}\left(W_{+} ; \mathrm{Cl}_{n+1}\right)}{\mathrm{C}_{\Gamma}^{*} \mathrm{~L}\left(X \subset W_{+} ; \mathrm{Cl}_{n+1}\right)}
$$

induced by inclusion is injective. Choose an appropriate homotopy equivalence $\psi: \mathcal{S} \rightarrow \mathcal{S}(-\varepsilon, \varepsilon)$ such that $\alpha \circ \psi$ and $\widetilde{\alpha} \circ \psi$ take values in $\mathrm{C}_{\Gamma}^{*} \mathrm{~L}_{Z}\left(W ; \mathrm{Cl}_{n+1}\right)$ and $\mathrm{C}_{\Gamma}^{*} \mathrm{~L}_{\widetilde{Z}}\left(\widetilde{W} ; \mathrm{Cl}_{n+1}\right)$, respectively. We also consider the quotient map

$$
\pi_{Z}: \mathrm{C}_{\Gamma}^{*} \mathrm{~L}_{Z}\left(W ; \mathrm{Cl}_{n+1}\right) \rightarrow \frac{\mathrm{C}_{\Gamma}^{*} \mathrm{~L}_{Z}\left(W ; \mathrm{Cl}_{n+1}\right)}{\mathrm{C}_{\Gamma}^{*} \mathrm{~L}_{Z \cap W_{-}}\left(W_{-} \subset W ; \mathrm{Cl}_{n+1}\right)},
$$


and define $\tilde{\pi}_{Z}$ analogously. We then have

$$
k \circ j^{-1} \circ \pi_{Z} \circ \varphi \circ \psi=\Phi \circ \pi \circ \varphi \circ \psi=\widetilde{\Phi} \circ \widetilde{\pi} \circ \widetilde{\varphi} \circ \psi=k \circ \tilde{j}^{-1} \circ \tilde{\pi}_{Z} \circ \widetilde{\varphi} \circ \psi
$$

Since $k$ is injective,

$$
j^{-1} \circ \pi_{Z} \circ \varphi \circ \psi=\widetilde{j}^{-1} \circ \tilde{\pi}_{Z} \circ \widetilde{\varphi} \circ \psi: \mathcal{S} \rightarrow \frac{\mathrm{C}_{\Gamma}^{*} \mathrm{~L}_{Z \cap W_{+}}\left(W_{+} ; \mathrm{Cl}_{n+1}\right)}{\mathrm{C}_{\Gamma}^{*} \mathrm{~L}_{Z \cap X}\left(X \subset W_{+} ; \mathrm{Cl}_{n+1}\right)} .
$$

This proves that

$$
\begin{aligned}
\left(j^{-1} \circ \pi_{Z}\right)_{*} \rho_{Z}^{\Gamma}(h) & =\left(\widetilde{j}^{-1} \circ \widetilde{\pi}_{Z}\right)_{*} \rho_{\widetilde{Z}}^{\Gamma}(\widetilde{h}) \\
& \in \mathrm{K}_{n+1}\left(\frac{\mathrm{C}_{\Gamma}^{*} \mathrm{~L}_{Z \cap W_{+}}\left(W_{+}\right)}{\mathrm{C}_{\Gamma}^{*} \mathrm{~L}_{Z \cap X}\left(X \subset W_{+}\right)}\right) \stackrel{\partial_{W_{+}}}{\rightarrow} \mathrm{K}_{n}\left(\mathrm{C}_{\Gamma}^{*} \mathrm{~L}_{Z \cap X}\left(X \subset W_{+}\right)\right) .
\end{aligned}
$$

This implies the desired statement, since the Mayer-Vietoris boundary maps can be computed by composing $\left(j^{-1} \circ \pi_{Z}\right)_{*}$ (respectively $\left.\left(\widetilde{j}^{-1} \circ \tilde{\pi}_{Z}\right)_{*}\right)$ with the boundary map $\partial_{W_{+}}$displayed above, see Remark 1.1.8.

Finally, to complete the proof we need to verify (2.4.9). We proceed similarly as in [PS14, p. 992]. Let $u \in \mathfrak{H}_{+}=\mathrm{L}^{2}\left(W_{+}, \Phi_{h}\right)=\mathrm{L}^{2}\left(\widetilde{W}_{+}, \Phi_{\widetilde{h}}\right)$ such that $\operatorname{supp}(u) \cap$ $\mathcal{U}_{\varepsilon}(X)=\emptyset$ for some $\varepsilon>0$. Since the Dirac wave operators have unit propagation speed and $\mathfrak{D}_{h}=\mathscr{D}_{\tilde{h}}$ on $W_{+}$, it follows that

$$
\mathrm{e}^{\mathrm{i} t \mathfrak{D}_{h}} u=\mathrm{e}^{\mathrm{i} t \not_{\tilde{h}}} u \in \mathfrak{H}_{+}
$$

for all $|t|<\varepsilon$, compare [HR00, Corollary 10.3.4]. Now let $f \in \mathcal{S}$ with compactly supported Fourier transform $\hat{f}$. Choose $R>0$ such that $\operatorname{supp}(\hat{f}) \subseteq[-R, R]$. Set $f_{t}(x):=f\left(t^{-1} x\right)$, then $\operatorname{supp}\left(\hat{f}_{t}\right) \subseteq\left[-\frac{R}{t}, \frac{R}{t}\right]$. Using the Fourier inversion formula à la [HR00, Proposition 10.3.5] together with (2.4.11), we obtain

$$
f\left(\frac{1}{t} \mathscr{D}_{h}\right) u=\int_{-R / t}^{R / t} \hat{f}_{t}(s) \mathrm{e}^{\mathrm{i} s \not_{h}} u \mathrm{~d} s=\int_{-R / t}^{R / t} \hat{f}_{t}(s) \mathrm{e}^{\mathrm{i} s \not_{\tilde{h}}} u \mathrm{~d} s=f\left(\frac{1}{t} \mathscr{D}_{\tilde{h}}\right) u,
$$

whenever $\operatorname{supp}(u) \cap \mathcal{U}_{R / t}(X)=\emptyset$. Let $N \in \mathbf{C L}\left[W_{+} ; \mathrm{Cl}_{n}\right]^{\Gamma}$ denote the family of operators defined by (2.4.9). We have just shown that $\operatorname{supp}(N(t)) \subseteq W_{+} \times \mathcal{U}_{R / t}(X)$ for all $t \geqslant 1$. Since $\operatorname{prop}(N(t)) \rightarrow 0$ as $t \rightarrow \infty$, this already proves $N \in \mathbf{C L}\left[X \subset W_{+} ; \mathrm{Cl}_{n}\right]^{\Gamma}$. Therefore (2.4.9) holds for all $f \in \mathcal{S}$ with compactly supported Fourier transform. The general statement follows since those functions are dense in $\mathcal{S}$.

Proof of Theorem 2.4.6. Applying Lemma 2.4.11 twice reduces the theorem to the product situation: Indeed, we consider a new partitioned manifold $\widetilde{W}$ with $\widetilde{W_{+}}=W_{+}$, $\widetilde{W}_{-}=X \times(-\infty, 0], \widetilde{Z} \cap W_{+}=Z \cap W_{+}, \widetilde{Z} \cap W_{-}=Z \cap X \times(-\infty, 0]$ and $h=g \oplus \mathrm{d} t^{2}$ on $W_{-}$. We also need to construct a distance function $d_{\widetilde{W}}$. To obtain that, we just glue the Euclidean product distance function on $X \times(-\infty, 0]$ to the metric on $W_{+}$ (where we use the restriction of $d_{W}$ ) as in Definition 1.3.7. Then, by Lemma 2.4.11 it 
suffices to show the partitioned manifold index theorem for $\widetilde{W}$ instead of $W$. However, in this case $\widetilde{Z}$ is also admissible with respect to $\widetilde{W}_{-}$, so we can swap the roles of $W_{+}$ and $W_{-}$in Lemma 2.4 .11 to also replace $W_{+}$by $X \times[0, \infty)$. Thus we only need to consider $W=X \times \mathbb{R}, Z=Z \cap X \times \mathbb{R}, h=g \oplus \mathrm{d} t^{2}$. This special case of the theorem was already proved in Theorem 2.3.9.

\subsubsection{The coarse APS-index theorem}

In this subsection, we derive an index theorem that relates the coarse APS-index with the partial $\rho$-invariant at the boundary together with a new $\rho$-invariant associated to a "coarse null-bordism". This constitutes a generalization of the "delocalized APS-index theorem" due to Piazza-Schick [PS14].

To begin, we suppose that we are in a geometric setup as in the definition of a coarse APS-index, see Subsection 2.2.3. That is, fix $Y$ to be an $(n+1)$-dimensional complete spin manifold with boundary $\partial Y=X$ endowed with a $\Gamma$-action. Moreover, we assume that $X \hookrightarrow Y$ is a coarse equivalence.

Lemma 2.4.12. The $\mathrm{K}$-theory of $\mathrm{C}_{\Gamma}^{*}\left(Y_{\infty}\right)$ vanishes in all degrees. In particular, there exists a unique element $\rho^{\Gamma}\left(Y_{\infty}\right) \in \mathrm{S}_{*}^{\Gamma}\left(Y_{\infty}\right)$ which maps to $\left[Y_{\infty}\right]^{\Gamma} \in \mathrm{K}_{*}^{\Gamma}\left(Y_{\infty}\right)$.

Proof. By assumption, $Y_{\infty}$ is coarsely equivalent to $X \times \mathbb{R}_{\geqslant 0}$ and thus the first statement follows from Corollary 2.1.14. The second statement is due to exactness.

Definition 2.4.13. We define

$$
\rho^{\Gamma}(Y):=\partial_{\mathrm{MV}}\left(\rho^{\Gamma}\left(Y_{\infty}\right)\right) \in \mathrm{S}_{n}^{\Gamma}(X),
$$

where $\rho^{\Gamma}\left(W_{\infty}\right)$ is as in Lemma 2.4.12 and we use the Mayer-Vietoris boundary map associated to the cover $W_{\infty}=W \cup\left(X \times \mathbb{R}_{\geqslant 0}\right)$.

Remark 2.4.14. Theorem 2.4.6 (applied to $Y_{\infty}$ with $Z=Y_{\infty}$ ) and naturality of the Mayer-Vietoris sequence show that $\rho^{\Gamma}(Y)$ maps to $[X]^{\Gamma} \in \mathrm{K}_{n}^{\Gamma}(X)$. Since the Khomology fundamental class of a spin manifold does not vanish (see for instance [HR00, Lemma 12.2.4]), $\rho^{\Gamma}(Y) \in \mathrm{S}_{n}^{\Gamma}(X)$ never vanishes either. However, $\operatorname{Ind}^{\Gamma}(X)$ vanishes by exactness. This constitutes a variant of bordism invariance for the coarse index, compare [Wul12].

We may think of the element $\rho^{\Gamma}(Y) \in \mathrm{S}_{n}^{\Gamma}(X)$ as a secondary invariant which is associated to $Y$ viewed as a null-bordism for $X$.

For each $g \in \mathcal{R}_{Z}^{+}(X)^{\Gamma}$, there is a secondary invariant $\rho_{Z}^{\Gamma}(g) \in \mathrm{S}_{n}^{\Gamma}(X / / Z)$. In the following, we will identify the difference between $\rho_{Z}^{\Gamma}\left(g_{X}\right)$ and $\rho^{\Gamma}(Y)$ as determined by the coarse APS-index from Subsection 2.2.3.

Theorem 2.4.15. For every $g \in \mathcal{R}_{Z}^{+}(X)^{\Gamma}$ the following identity holds:

$$
\partial_{/ / Z}^{\Gamma}\left(\operatorname{Ind}_{\mathrm{APS}}^{\Gamma}(Y, X / / Z, g)\right)=\rho_{Z}^{\Gamma}(g)-q_{0, Z}^{\Gamma}\left(\rho^{\Gamma}(Y)\right) \in \mathrm{S}_{n}^{\Gamma}(X / / Z) .
$$

Here we have used the notation from Corollary 2.1.4. In particular, for $Z=\emptyset$ : 
Corollary 2.4.16. For every $g \in \mathcal{R}^{+}(X)^{\Gamma}$ the following identity holds:

$$
\partial^{\Gamma}\left(\operatorname{Ind}_{\mathrm{APS}}^{\Gamma}(Y, X, g)\right)=\rho^{\Gamma}(g)-\rho^{\Gamma}(Y) \in \mathrm{S}_{n}^{\Gamma}(X) .
$$

Proof of Theorem 2.4.15. As a minor deviation from the setup of Subsection 2.2.3, we redefine $Z_{\infty}$ as

$$
Z_{\infty}:=(Y \backslash(X \times(-\varepsilon, 0])) \cup(Z \times(-\varepsilon, \infty)) \subseteq Y_{\infty},
$$

where we implicitly use the fixed collar neighborhood of $X$ and some sufficiently small $\varepsilon>0$. This does not change any of the invariants since it is coarsely equivalent to the variant of $Z_{\infty}$ defined in Subsection 2.2.3 but has the advantage that it is admissible with respect to $X \times \mathbb{R}_{\geqslant 0}$ in the partitioned manifold $Y_{\infty}=Y \cup X \times \mathbb{R}_{\geqslant 0}$. In particular, we will be able to apply Theorem 2.4.6 to this situation.

Choose $h \in \mathcal{R}_{Z}^{+}(Y)^{\Gamma}$ with $\partial h=g$. Lemma 2.4 .12 gives maps

$$
\begin{gathered}
r: \mathrm{S}_{*}^{\Gamma}\left(Y_{\infty} / / Z_{\infty}\right) \rightarrow \mathrm{K}_{*}^{\Gamma}\left(Y_{\infty}\right) \cong \mathrm{S}_{*}^{\Gamma}\left(Y_{\infty}\right), \\
s: \mathrm{K}_{*}\left(\mathrm{C}_{\Gamma}^{*}\left(Z_{\infty} \subset W\right)\right) \cong \mathrm{K}_{*+1}\left(\frac{\mathrm{C}_{\Gamma}^{*}\left(Y_{\infty}\right)}{\mathrm{C}_{\Gamma}^{*}\left(Z_{\infty} \subset Y_{\infty}\right)}\right) \stackrel{\delta}{\rightarrow} \mathrm{S}_{*}^{\Gamma}\left(Y_{\infty} / / Z_{\infty}\right),
\end{gathered}
$$

where $\delta=\partial_{\| / Z_{\infty}}^{\Gamma}$. By construction, $r\left(\rho_{Z_{\infty}}^{\Gamma}\left(h_{\infty}\right)\right)=\rho^{\Gamma}\left(Y_{\infty}\right)$ and $\operatorname{Ind}_{Z_{\infty}}^{\Gamma}\left(\rho_{Z_{\infty}}^{\Gamma}\left(h_{\infty}\right)\right)=$ $\operatorname{Ind}_{Z_{\infty}}^{\Gamma}\left(Y_{\infty}, h_{\infty}\right)$. Let $i:=q_{0, Z_{\infty}}^{\Gamma}: \mathrm{S}_{*}^{\Gamma}\left(Y_{\infty}\right) \rightarrow \mathrm{S}_{*}^{\Gamma}\left(Y_{\infty} / / Z_{\infty}\right)$ and $j:=q_{0, Z}^{\Gamma}: \mathrm{S}_{*}^{\Gamma}(X) \rightarrow$ $\mathrm{S}_{*}^{\Gamma}(X / / Z)$. We are in the following situation:

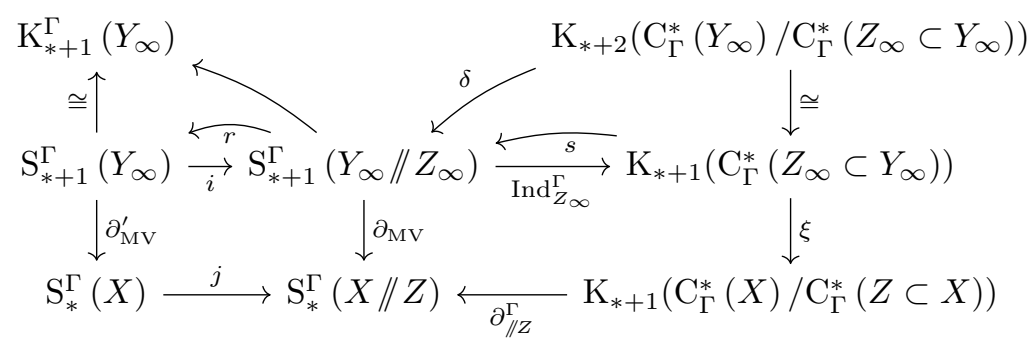

Here $\xi:=\zeta \circ \pi_{Z \times \mathbb{R}_{\geqslant 0}}$, where $\pi_{Z \times \mathbb{R}_{\geqslant 0}}$ is induced by the quotient map $\mathrm{C}_{\Gamma}^{*}\left(Z_{\infty}\right) \rightarrow$ $\mathrm{C}_{\Gamma}^{*}\left(Z_{\infty}\right) / \mathrm{C}_{\Gamma}^{*}\left(Z \times \mathbb{R}_{\geqslant 0} \subset Z_{\infty}\right)$ and $\zeta$ is defined as in Subsection 2.2.3.

One can verify that

$$
r \circ i=\mathrm{id}, \quad \operatorname{Ind}_{Z_{\infty}}^{\Gamma} \circ s=\mathrm{id}, \quad r \circ s=0 .
$$

Hence $s$ (respectively $r$ ) splits the long exact sequence $\mathrm{S}_{*}^{\Gamma}\left(Y_{\infty}\right) \rightarrow \mathrm{S}_{*}^{\Gamma}\left(Y_{\infty} / / Z_{\infty}\right) \rightarrow$ $\mathrm{K}_{*}\left(\mathrm{C}_{\Gamma}^{*}\left(Z_{\infty} \subset Y_{\infty}\right)\right)$. In particular, $i \circ r+s \circ \operatorname{Ind}_{Z_{\infty}}^{\Gamma}=\mathrm{id}$ and thus

$$
\rho_{Z_{\infty}}^{\Gamma}\left(h_{\infty}\right)=i\left(\rho^{\Gamma}\left(Y_{\infty}\right)\right)+s\left(\operatorname{Ind}_{Z_{\infty}}^{\Gamma}\left(Y_{\infty}, h_{\infty}\right)\right) .
$$

Additional diagram chases show that

$$
\begin{aligned}
& \partial_{\mathrm{MV}} \circ i=j \circ \partial_{\mathrm{MV}}^{\prime}, \\
& \partial_{\mathrm{MV}} \circ s=\partial_{/ / Z}^{\Gamma} \circ \xi,
\end{aligned}
$$


Figure 2.2: Diagram needed to verify (2.4.12)

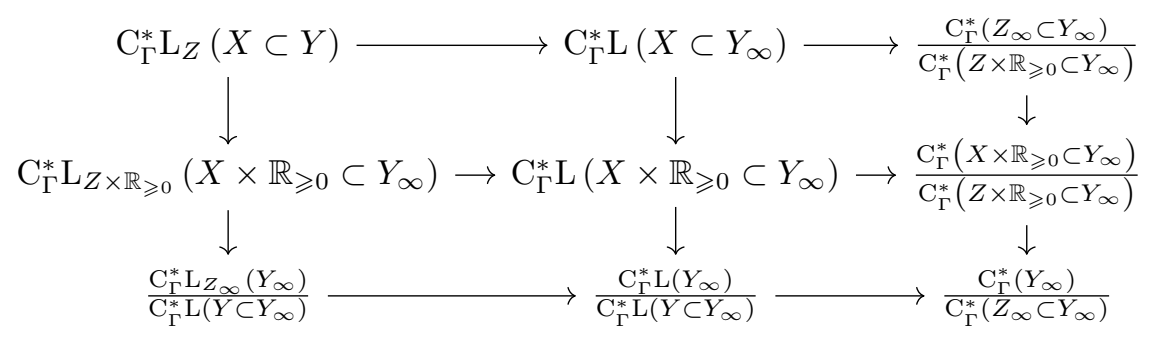

where the latter can be proved using the grid of exact sequences displayed in Figure 2.2. Hence

$$
\begin{aligned}
\partial_{\mathrm{MV}}\left(\rho_{Z_{\infty}}^{\Gamma}\left(h_{\infty}\right)\right) & =\left(j \circ \partial_{\mathrm{MV}}^{\prime}\right)\left(\rho^{\Gamma}\left(Y_{\infty}\right)\right)+\left(\partial_{/ / Z}^{\Gamma} \circ \xi\right)\left(\operatorname{Ind}_{Y}^{\Gamma}\left(Y_{\infty}, h_{\infty}\right)\right. \\
& =j\left(\rho^{\Gamma}(Y)\right)+\partial_{/ / Z}^{\Gamma}\left(\operatorname{Ind}_{\mathrm{APS}}^{\Gamma}(Y, X / / Z, g)\right) .
\end{aligned}
$$

Finally, we apply Theorem 2.4 .6 to $Y_{\infty}$ partitioned by $X$ and with $Z=Z_{\infty}$ to obtain $\partial_{\mathrm{MV}}\left(\rho_{Y}^{\Gamma}\left(h_{\infty}\right)\right)=\rho_{Z}^{\Gamma}\left(g_{X}\right)$. This concludes the proof.

Corollary 2.4.17. Let $\iota: X \hookrightarrow Y$ denote the inclusion map. Then

$$
\iota_{*} \partial_{/ / Z}^{\Gamma}\left(\operatorname{Ind}_{\mathrm{APS}}^{\Gamma}(Y, X / / Z, g)\right)=\iota_{*} \rho_{Z}^{\Gamma}(g) \in \mathrm{S}_{n}^{\Gamma}(Y / / Z) .
$$

Proof. By construction, $\rho^{\Gamma}(Y)$ lies in the kernel of $\iota_{*}: \mathrm{S}_{n}^{\Gamma}(X) \rightarrow \mathrm{S}_{n}^{\Gamma}(Y)$ and thus the corollary is a consequence of Theorem 2.4.15.

Corollary 2.4.18 ([PS14]). Suppose that $Z=\emptyset$ and let $\iota: X \hookrightarrow Y$ denote the inclusion map. Then:

$$
\iota_{*} \partial^{\Gamma}\left(\operatorname{Ind}_{\mathrm{APS}}^{\Gamma}(Y, X, g)\right)=\iota_{*} \rho^{\Gamma}(g) \in \mathrm{S}_{n}^{\Gamma}(Y) .
$$

Corollary 2.4.19. Let $g_{0}, g_{1} \in \mathcal{R}_{Z}^{+}(X)^{\Gamma}$. Then

$$
\partial_{/ / Z}^{\Gamma}\left(\operatorname{Ind}_{\text {diff }}^{\Gamma}\left(g_{0}, g_{1} / / Z\right)\right)=\rho_{Z}^{\Gamma}\left(g_{0}\right)-\rho_{Z}^{\Gamma}\left(g_{1}\right) \in \mathrm{S}_{n}^{\Gamma}(X / / Z) .
$$

Proof. It follows from the definition of the index difference that

$$
\operatorname{Ind}_{\text {diff }}^{\Gamma}\left(g_{0}, g_{1} / / Z\right)=\left(\operatorname{pr}_{X}\right)_{*} \iota_{*} \operatorname{Ind}_{\text {APS }}^{\Gamma}\left(X \times[0,1], X \times\{0,1\} / / Z \times\{0,1\}, g_{0} \sqcup g_{1}\right) .
$$

Hence Corollary 2.4.17 gives

$$
\begin{aligned}
\partial_{X / / Z}^{\Gamma} \operatorname{Ind}_{\mathrm{diff}}^{\Gamma}\left(g_{0}, g_{1} / / Z\right) \\
=\left(\operatorname{pr}_{X}\right)_{*} \iota_{*} \partial_{X \times\{0,1\} / / Z \times\{0,1\}}^{\Gamma}\left(\operatorname{Ind}_{\mathrm{APS}}^{\Gamma}\left(X \times[0,1], X \times\{0,1\} / / Z \times\{0,1\}, g_{0} \sqcup g_{1}\right)\right) \\
=\left(\operatorname{pr}_{X}\right)_{*} \iota_{*} \rho_{Z \times\{0,1\}}^{\Gamma}\left(g_{0} \sqcup g_{1}\right)=\rho_{Z}^{\Gamma}\left(g_{0}\right)-\rho_{Z}^{\Gamma}\left(g_{1}\right) .
\end{aligned}
$$


The next corollary shows that the partial $\rho$-invariant is a bordism invariant in a suitable sense.

Corollary 2.4.20. In the situation of Theorem 2.4.15, suppose that there exists a $\Gamma$-invariant subset $A \subseteq Y$ such that $\sup _{z \in Z} d_{Y}(z, A)<\infty$ and $h \in \mathcal{R}_{A}^{+}(Y)^{\Gamma}$ with $\partial h=g$. Then

$$
\iota_{*} \rho_{Z}^{\Gamma}(g)=0 \in \mathrm{S}_{n}^{\Gamma}(Y / / Z) .
$$

Proof. This is an immediate consequence of Proposition 2.2.19 and Corollary 2.4.17.

\subsection{Explicit descriptions in terms of projections and unitaries}

In this section, we focus on the complex case. We now use the notation $K U_{j}(A)$, $j \in \mathbb{Z}_{2}$, to denote "ordinary" complex K-theory of an ungraded complex $\mathrm{C}^{*}$-algebra $A$ defined in terms of projections and unitaries (as a reference see for example [Weg93]).

We describe the partial $\rho$-invariants in $K U_{0}\left(\mathrm{C}_{\Gamma}^{*} \mathrm{~L}_{Z}(X)\right)$ and $K U_{1}\left(\mathrm{C}_{\Gamma}^{*} \mathrm{~L}_{Z}(X)\right)$. This is done in essentially the same way as Xie-Yu [XY14b] define the "local index class" and the $\rho$-invariant. We show that this agrees with the elements defined in Subsection 2.2.2 up to a sign and a natural isomorphism $\mathrm{K}_{0}\left(A \widehat{\otimes} \mathbb{C l}_{n}\right) \cong K U_{n}(A)$ for ungraded $\mathrm{C}^{*}$ algebras $A$.

We drop the group action to simplify the notation, but it would not entail any additional technical difficulties to include it.

\subsubsection{K-theory of trivially graded $\mathrm{C}^{*}$-algebras}

We need an explicit isomorphism between (the complex version of) the picture of K-theory explained in Section 1.1 and complex K-theory for trivially graded algebras defined in terms of projections and unitaries.

Proposition 2.5.1 ([HG04, p.149f]). For every trivially graded $\mathrm{C}^{*}$-algebra $A$ there are natural isomorphisms $\Theta_{2 k}: \mathrm{K}_{0}\left(A \widehat{\otimes} \mathbb{C l}_{2 k}\right) \rightarrow K U_{0}(A)$ and $\Theta_{2 k+1}: \mathrm{K}_{0}\left(A \widehat{\otimes} \mathbb{C l}_{2 k+1}\right) \rightarrow$ $K U_{1}(A)$ such that the following diagram of isomorphisms commutes,

$$
\begin{gathered}
\mathrm{K}_{0}\left(A \widehat{\otimes} \mathbb{C l}_{2 k+1}\right) \stackrel{\times b}{\longrightarrow} \mathrm{K}_{0}\left(\mathcal{C}_{0}(\mathbb{R}) \otimes A \widehat{\otimes} \mathbb{C l}_{2 k}\right) \\
\downarrow \Theta_{2 k+1} \\
K U_{1}(A) \stackrel{\delta}{\longrightarrow} K_{0}\left(\mathcal{C}_{0}(\mathbb{R}) \otimes A\right),
\end{gathered}
$$

where $\delta$ is the standard suspension isomorphism in K-theory and $\times b$ is the Bott isomorphism from Subsection 1.1.3.

Proof. Since by (formal) periodicity $\mathrm{K}_{0}\left(A \widehat{\otimes} \mathbb{C l}_{2 k}\right)=\mathrm{K}_{0}(A)$ and $\mathrm{K}_{0}\left(A \widehat{\otimes} \mathbb{C l}_{2 k+1}\right)=$ $\mathrm{K}_{0}\left(A \widehat{\otimes} \mathbb{C l}_{1}\right)$, we can restrict ourselves to the case $k=0$. Let $\varphi: \mathcal{S} \rightarrow A \widehat{\otimes} \mathbb{K}$ be given and consider the unitary,

$$
U_{\varphi}:=\varphi^{+}\left(\frac{\mathrm{x}-\mathrm{i}}{\mathrm{x}+\mathrm{i}}\right) \in(A \widehat{\otimes} \mathbb{K})^{+},
$$


where $(A \widehat{\otimes} \mathbb{K})^{+}$denotes the unitization of $A \widehat{\otimes} \mathbb{K}$. Observe that $U_{\varphi}$ is equal to the identity modulo $A \widehat{\otimes} \mathbb{K}$. Since $A$ is trivially graded, the ungraded tensor product $A \otimes \mathbb{K}$ coincides with $A \widehat{\otimes} \mathbb{K}$ when we neglect the grading. We will use the symbol $A \otimes \mathbb{K}$ if we wish to consider it as an ungraded algebra, and $A \widehat{\otimes} \mathbb{K}$ if we want to emphasize the grading. Using this convention, the graded $\mathrm{C}^{*}$-algebra $A \widehat{\otimes} \mathbb{K}$ can be identified with the matrix algebra $\mathrm{M}_{2}(A \otimes \mathbb{K})$, where the grading automorphisms is conjugation with the multiplier

$$
\epsilon=\left(\begin{array}{cc}
1 & 0 \\
0 & -1
\end{array}\right)
$$

Then we have

$$
\epsilon U_{\varphi} \epsilon=U_{\varphi}^{*}
$$

In particular, $\epsilon U_{\varphi}$ is a self-adjoint unitary in $\mathrm{M}_{2}\left((A \otimes \mathbb{K})^{+}\right)$eqal to $\epsilon$ modulo $A \otimes \mathbb{K}$. As a consequence, $\mathrm{P}_{\varphi}:=\frac{1}{2}\left(1+\epsilon U_{\varphi}\right)$ is a projection in $\mathrm{M}_{2}\left((A \otimes \mathbb{K})^{+}\right)$equal to $\mathrm{P}_{\epsilon}:=$ $\frac{1}{2}(1+\epsilon)$ modulo $A \otimes \mathbb{K}$. We define $\Theta_{0}([\varphi]):=\left[P_{\varphi}\right]-\left[P_{\epsilon}\right] \in K U_{0}(A \otimes \mathbb{K})=K U_{0}(A)$.

Similarly, if we have a $*$-homomorphism $\varphi: \mathcal{S} \rightarrow A \widehat{\otimes}\left(\mathbb{C l}_{1} \widehat{\otimes} \mathbb{K}\right)$, we again form the unitary $U_{\varphi}=\varphi^{+}\left(\frac{\mathrm{x}-\mathrm{i}}{\mathrm{x}+\mathrm{i}}\right) \in\left(A \widehat{\otimes} \mathbb{C l}_{1} \widehat{\otimes} \mathbb{K}\right)^{+}$. Using the explicit description of the Clifford algebra $\mathbb{C l}_{1} \cong \mathbb{C} \oplus \mathbb{C}$, we may consider $U_{\varphi, 1}=\operatorname{pr}_{1}^{+}\left(U_{\varphi}\right)$, a unitary in $(A \otimes \mathbb{K})^{+}$. We define $\Theta_{1}([\varphi]):=\left[U_{\varphi, 1}\right] \in K U_{1}(A \otimes \mathbb{K})=K U_{1}(A)$.

The maps $\Theta_{i}$ defined above are well-defined because if $\varphi$ and $\psi$ are homotopic, then so are $U_{\varphi}$ and $U_{\psi}$. Since the additive structure on $\mathrm{K}_{0}$ is essentially defined by block sum inside $A \widehat{\otimes} \mathbb{K}$, the maps $\Theta_{n}$ are additive. Moreover, it follows from [HG04, Lemma 1.4] that these maps are isomorphisms.

Finally, due to naturality, it suffices to consider the case $A=\mathcal{C}_{0}(\mathbb{R})$ to show that the diagram in the proposition commutes. The group $K U_{0}\left(\mathcal{C}_{0}(\mathbb{R}) \widehat{\otimes} \mathbb{C l}_{1}\right)$ is generated by the Bott element $b$ which is represented by the $*$-homomorphism $\beta: \mathcal{S} \rightarrow \mathcal{C}_{0}\left(\mathbb{R}, \mathbb{C l} l_{1}\right) \cong$ $\mathcal{C}_{0}(\mathbb{R}) \oplus \mathcal{C}_{0}(\mathbb{R}), f \mapsto(x \mapsto(f(x), f(-x)))$. Thus $\Theta_{1}(b)$ is represented by the unitary $\frac{\mathrm{x}-\mathrm{i}}{\mathrm{x}+\mathrm{i}}$ in $\mathcal{C}_{0}(\mathbb{R})^{+}$which has winding number +1 and thus represents the standard generator of $K U_{1}\left(\mathcal{C}_{0}(\mathbb{R})\right)$. Thus $\delta\left(\Theta_{1}(b)\right) \in K U_{0}\left(\mathcal{C}_{0}\left(\mathbb{R}^{2}\right)\right)$ is represented by the Bott projection. The element $b \times b \in \mathrm{K}_{0}\left(\mathcal{C}_{0}\left(\mathbb{R}^{2}\right)\right)$ is represented by the $*$-homomorphism

$$
\varphi: \mathcal{S} \rightarrow \mathcal{C}_{0}\left(\mathbb{R}^{2}, \mathrm{M}_{2}(\mathbb{C})\right), \quad f \mapsto f\left(\left(\begin{array}{cc}
0 & \mathrm{x}+\mathrm{iy} \\
\mathrm{x}-\mathrm{iy} & 0
\end{array}\right)\right) .
$$

A direct computation shows that $P_{\varphi}$ is also the Bott projection, hence the diagram commutes.

\subsubsection{Reduced spinor bundles}

So far, we have worked with the $\mathbb{C l}_{n}$-linear (or $n$-multi-graded in the terminology of [HR00]) spinor bundle and Dirac operator. Here we review the equivalent viewpoint using irreducible Clifford modules. If $n$ is even, there is up to isomorphism only one irreducible Clifford module, which we denote by $\$(n)$. It automatically carries a grading $\$(n)=\$^{(0)}(n) \oplus \$^{(1)}(n)$. If $n$ is odd, there are two irreducible Clifford 
modules $\$_{+}(n)$ and $\$_{-}(n)$ which are ungraded. Let $X$ be a spin manifold with Riemannian metric $g$. Then the reduced spinor bundle is the associated bundle $\$_{g}=\mathrm{P}_{\text {Spin }}\left(\mathrm{T}^{*} X\right) \times_{\operatorname{Spin}(n)} \$(n)$. In the odd-dimensional case the representations of $\operatorname{Spin}(n)$ coming from the two irreducible Clifford modules are isomorphic, so it does not matter which we choose. We may realize $\$(n)$ concretely as a left ideal inside $\mathbb{C l}_{n}$, so that $\$_{g}$ is a sub-bundle of $\Phi(X)$ which is $\mathbb{C l}\left(\mathrm{T}^{*} X\right)$-invariant. In particular, the $\mathbb{C l}_{n}$-linear Dirac operator $\not{D}$ restricts to the spinor Dirac operator $\not D$ on $\$_{g}$. Let $\mathfrak{H}:=\mathrm{L}^{2}\left(X, \Phi_{g}\right)$ and $\mathcal{H}:=\mathrm{L}^{2}\left(X, \$_{g}\right)$. The first is always a graded Hilbert $\mathbb{C l}_{n}$-module whereas the latter is a Hilbert space, furnished with a grading if and only if $n$ is even.

Suppose $n=2 k>0$. Then $\operatorname{End}_{\mathbb{C}}(\$(n))=\mathbb{C l}_{n}$ and there is a one to one correspondence between (possibly unbounded) $\mathbb{C l}_{n}$-linear operators on $\mathfrak{H}$ and $\mathbb{C}$-linear operators on $\mathcal{H}$. Indeed, every $\mathbb{C l}_{n}$-linear operator on $\mathfrak{H}$ keeps $\mathcal{H}$ invariant and is uniquely determined by its restiction to $\mathcal{H}$. On the level of Roe algebras this yields a canonical isomorphism (of graded $\mathrm{C}^{*}$-algebras),

$$
\mathrm{C}^{*}\left(X, \mathfrak{H} ; \mathbb{C l}_{2 k}\right)=\mathrm{C}^{*}(X, \mathcal{H}) \text {. }
$$

In the odd-dimensional case $n=2 k+1$, we have $\mathbb{C l}_{n}=\operatorname{End}_{\mathbb{C}}\left(\$_{+}(n)\right) \oplus \operatorname{End}_{\mathbb{C}}\left(\$_{-}(n)\right)$. A similar argument as above yields,

$$
\mathrm{C}^{*}\left(X, \mathfrak{H} ; \mathbb{C l}_{2 k+1}\right)=\mathrm{C}^{*}(X, \mathcal{H}) \oplus \mathrm{C}^{*}(X, \mathcal{H}) .
$$

The identifications (2.5.2) and (2.5.3) hold analogously for the structure algebra $\mathrm{D}^{*}(X)$ and the all localization algebras and $\mathrm{C}^{*} \mathrm{~L}_{Z}(X)$ and $\mathrm{D}^{*} \mathrm{~L}(X)$.

\subsubsection{Local index classes in terms of projections and unitaries}

A normalizing function is a continuous odd non-decreasing function $\chi: \mathbb{R} \rightarrow[-1,1]$ such that $\lim _{x \rightarrow \pm \infty} \chi(x)= \pm 1$. Let $L_{\not D}:[1, \infty) \rightarrow \mathbf{D}[X, \mathcal{H}], L_{\not D}(t)=\chi\left(\frac{1}{t} \not D\right)$, where $\chi$ is a normalizing function. Let $Z \subseteq X$ be a closed subset (possibly $Z=\emptyset$ or $Z=X$ ) such that the scalar curvature function on $X$ is uniformly positive outside $Z$. Then Lemma 2.2.5 implies $L_{\not D}^{2}-1 \in \mathrm{C}^{*} \mathrm{~L}_{Z}(X, \mathcal{H})$ provided that we have chosen $\chi$ such that $\chi^{2}=1$ outside $(-\varepsilon, \varepsilon)$.

If $n>0$ is even, then $\mathcal{H}$ is graded and $L_{\not D}(t)$ is an odd operator for all $t$, that is, with respect to the grading $\mathcal{H}=\mathcal{H}^{(0)} \oplus \mathcal{H}^{(1)}$, we have

$$
L_{\not D}(t)=\left(\begin{array}{cc}
0 & L_{\not D}^{-}(t) \\
L_{\not D}^{+}(t) & 0
\end{array}\right) .
$$

Let $v: \mathcal{H}^{(0)} \rightarrow \mathcal{H}^{(1)}$ be a unitary which intertwines the $\mathcal{C}_{0}(X)$-representations (for instance, take $v$ to be Clifford multiplication with a measurable unit co-vector field). Then $v^{*} L_{\not D}^{+} \in \mathrm{D}^{*} \mathrm{~L}\left(X, \mathcal{H}^{(0)}\right)$ is a unitary modulo $\mathrm{C}^{*} \mathrm{~L}_{Z}\left(X, \mathcal{H}^{(0)}\right)$ and hence defines a class $\left[v^{*} L_{\not D}^{+}\right] \in K U_{1}\left(\mathrm{D}^{*} \mathrm{~L}(X) / \mathrm{C}^{*} \mathrm{~L}_{Z}(X)\right)$. We let

$$
\operatorname{Ind}_{\mathrm{L}, \mathrm{Z}}(\not D):=\partial\left[v^{*} L_{\not D}^{+}\right] \in K U_{0}\left(\mathrm{C}^{*} \mathrm{~L}_{Z}(X)\right)
$$


If $n$ is odd, then $\frac{1}{2}\left(1+L_{\not D}\right)$ is a projection modulo $\mathrm{C}^{*} \mathrm{~L}_{Z}(X, \mathcal{H})$, and we set

$$
\operatorname{Ind}_{\mathrm{L}, \mathrm{Z}}(\not D):=\partial\left[\frac{1}{2}\left(1+L_{\not D}\right)\right] \in K U_{1}\left(\mathrm{C}^{*} \mathrm{~L}_{Z}(X)\right) .
$$

Remark 2.5.2. For $Z=X$ (respectively $Z=\emptyset$ ), the element $\operatorname{Ind}_{\mathrm{L}, Z}(\not D)$ agrees with the local index class (respectively the $\rho$-invariant) defined in $[\mathrm{XY} 14 \mathrm{~b}]$. This can be proved using that the map $\mathrm{D}^{*} \mathrm{~L}(X) / \mathrm{C}^{*} \mathrm{~L}(X) \rightarrow \mathrm{D}^{*}(X) / \mathrm{C}^{*}(X)$ (respectively $\left.\mathrm{D}^{*} \mathrm{~L}(X) / \mathrm{C}^{*} \mathrm{~L}_{0}(X) \rightarrow \mathrm{D}^{*}(X)\right)$ defined by evaluation at $1 \in[1, \infty)$ is a $K U$-theory isomorphism, see [QR10].

Theorem 2.5.3. The isomorphism $\Theta_{n}$ from Proposition 2.5.1,

$$
\Theta_{n}: \mathrm{K}_{0}\left(\mathrm{C}^{*} \mathrm{~L}_{Z}\left(X ; \mathbb{C l}_{n}\right)\right)=\mathrm{K}_{0}\left(\mathrm{C}^{*} \mathrm{~L}_{Z}(X) \widehat{\otimes} \mathbb{C l}_{n}\right) \rightarrow K U_{n}\left(\mathrm{C}^{*} \mathrm{~L}_{Z}(X)\right),
$$

takes $\rho_{Z}(g)$ as defined in Subsection 2.2 .2 to $-\operatorname{Ind}_{\mathrm{L}, \mathrm{Z}}(\not D)$.

Proof. Let $\varepsilon>0$ such that $f(\not{D})$ and $f(\not D)$ lie in $\mathrm{C}^{*}(Z \subset X)$ for $f \in \mathcal{C}_{0}((-\varepsilon, \varepsilon))$ and choose a homotopy inverse $\psi: \mathcal{S} \rightarrow \mathcal{S}(-\varepsilon, \varepsilon)$ to the inclusion $\mathcal{S}(-\varepsilon, \varepsilon) \hookrightarrow \mathcal{S}$. Then $\operatorname{Ind}_{\mathrm{L}, \mathrm{Z}}(\mathscr{D})=\left[\varphi_{\not{D}} \circ \psi\right]$. We can choose $\psi$ in such a way that it extends to a homotopy equivalence $\bar{\psi}: \mathcal{C}([-\infty, \infty]) \rightarrow \mathcal{C}([-\varepsilon, \varepsilon])$, where we identify $\mathcal{C}([-\varepsilon, \varepsilon])$ with the subspace of $\mathcal{C}([-\infty, \infty])$ consisting of those functions which are constant on $[-\infty,-\varepsilon]$ as well as on $[\varepsilon, \infty]$. Let $\tilde{\chi}$ be the normalizing function $\tilde{\chi}(x):=\frac{x}{\sqrt{1+x^{2}}}$. We can assume that $\chi:=\bar{\psi}(\tilde{\chi})$ is still a normalizing function which by construction satisfies $\chi^{2}=1$ outside $(-\varepsilon, \varepsilon)$. Let $\tilde{\tau}(x):=\frac{x-\mathrm{i}}{\sqrt{1+x^{2}}}$, then $\tilde{\tau}(x)=\tilde{\chi}(x)-\frac{\mathrm{i}}{\sqrt{1+x^{2}}}$, so $\tilde{\chi} \equiv \tilde{\tau}$ modulo $\mathcal{C}_{0}(\mathbb{R})$. Set $\tau:=\bar{\psi}(\tilde{\tau})$, then $\chi \equiv \tau$ modulo $\mathcal{C}_{0}((-\varepsilon, \varepsilon))$. The unitary $U:=U_{\varphi_{\not \supset} \circ \psi}$ from Proposition 2.5.1 $1^{1}$ is given by $\varphi_{\not \supset}^{+}\left(\tau^{2}\right)$ since $\tilde{\tau}^{2}=\frac{\mathrm{x}-\mathrm{i}}{\mathrm{x}+\mathrm{i}}$. In particular, $U=T^{2}$, where $T(t):=\tau\left(\frac{1}{t} \mathfrak{D}\right)$ is a unitary in $\mathrm{D}^{*} \mathrm{~L}\left(X, \mathfrak{H} ; \mathrm{Cl}_{n}\right)$. Furthermore, $T(t)-\chi\left(\frac{1}{t} \mathfrak{D}\right)=(\tau-\chi)\left(\frac{1}{t} \mathfrak{D}\right)$, so $T-L_{\not{D}} \in \mathrm{C}^{*} \mathrm{~L}_{Z}\left(X, \mathfrak{H} ; \mathbb{C l}_{n}\right)$, where $L_{\mathfrak{D}}(t)=\chi\left(\frac{1}{t} \mathfrak{D}\right)$.

Suppose that $n>0$ is even. By (2.5.2), we have $\mathrm{C}^{*} \mathrm{~L}_{Z}\left(X, \mathfrak{H} ; \mathbb{C l}_{n}\right)=\mathrm{C}^{*} \mathrm{~L}_{Z}(X, \mathcal{H})$ and $L_{\not \supset}=L_{\not \supset} \in \mathrm{D}^{*} \mathrm{~L}\left(X, \mathfrak{H} ; \mathrm{Cl}_{n}\right)=\mathrm{D}^{*} \mathrm{~L}(X, \mathcal{H})$. Let $V:=\left(\begin{array}{cc}0 & v^{*} \\ v & 0\end{array}\right)$ and $W:=V T \in$ $\mathrm{D}^{*} \mathrm{~L}(X, \mathcal{H})$. Then $W$ is a unitary, which is equal to $V L_{\not D}=\left(\begin{array}{cc}v^{*} L_{\not D}^{+} & 0 \\ 0 & v L_{\not D}^{-}\end{array}\right)$modulo $\mathrm{C}^{*} \mathrm{~L}_{Z}(X, \mathcal{H})$. The definition of the boundary map in K-theory ([Weg93, Chapter 8.1]) gives

$$
\operatorname{Ind}_{\mathrm{L}, \mathrm{Z}}(\not D)=\partial\left[v^{*} L_{\not D}^{+}\right]=\left[W\left(\begin{array}{ll}
1 & 0 \\
0 & 0
\end{array}\right) W^{*}\right]-\left[\left(\begin{array}{ll}
1 & 0 \\
0 & 0
\end{array}\right)\right] \in K U_{0}\left(\mathrm{C}^{*} \mathrm{~L}_{Z}(X)\right) .
$$

Let $\epsilon=\left(\begin{array}{cc}1 & 0 \\ 0 & -1\end{array}\right)$ be the grading operator on $\mathcal{H}=\mathcal{H}^{(0)} \oplus \mathcal{H}^{(1)}$ and $P_{\epsilon}=\frac{1}{2}(1+\epsilon)=\left(\begin{array}{ll}1 & 0 \\ 0 & 0\end{array}\right)$. Since the complex conjugate of $\tilde{\tau}(x)$ is $-\tilde{\tau}(-x)$, we have $\epsilon T=-T^{*} \epsilon$. A direct

\footnotetext{
${ }^{1}$ We have enough room to carry out the construction of $\Theta_{n}$ from the proof of Proposition 2.5.1 inside $\mathrm{C}^{*} \mathrm{~L}_{Z}\left(X, \mathfrak{H} ; \mathbb{C l}_{n}\right)$ (without taking the tensor product with the compact operators) and we shall do so here.
} 
computation using this fact together with $U^{2}=T$ shows that $\frac{1}{2}(1+\epsilon U)=1-\epsilon T P_{\epsilon} T^{*} \epsilon$. Then we have

$$
\begin{aligned}
\Theta_{n}\left(\left[\varphi_{\not \supset} \circ \psi\right]\right) & =\left[\frac{1}{2}(1+\epsilon U)\right]-\left[P_{\epsilon}\right] \\
& =\left[1-\epsilon T P_{\epsilon} T^{*} \epsilon\right]-\left[P_{\epsilon}\right] \\
& =\left[P_{\epsilon}\right]-\left[\epsilon T P_{\epsilon} T^{*} \epsilon\right] \\
& =\left[P_{\epsilon}\right]-\left[W P_{\epsilon} W^{*}\right] \quad(\text { by conjugation with } V \epsilon) \\
& \left.=-\partial\left[v^{*} L_{\not D}^{+}\right]=-\operatorname{Ind}_{\mathrm{L}, \mathrm{Z}}(\not D) \quad \text { (by }(2.5 .4)\right) .
\end{aligned}
$$

If $n$ is odd, then $(2.5 .3)$ gives $\mathrm{C}^{*} \mathrm{~L}_{Z}\left(X, \mathfrak{H} ; \mathbb{C l}_{n}\right)=\mathrm{C}^{*} \mathrm{~L}_{Z}(X, \mathcal{H}) \oplus \mathrm{C}^{*} \mathrm{~L}_{Z}(X, \mathcal{H})$ and $\operatorname{pr}_{1}\left(L_{\not \supset}\right)=L_{\not D}$. Then

$$
\operatorname{Ind}_{\mathrm{L}, \mathrm{Z}}(\not D)=\partial\left[\frac{1}{2}\left(1+L_{\not \supset}\right)\right]=\left[\mathrm{e}^{-2 \pi \mathrm{i} \frac{1}{2}\left(1+\operatorname{pr}_{1}\left(L_{\not)}\right)\right)}\right]=\left[\operatorname{pr}_{1}^{+} \varphi_{\not \supset}^{+}\left(\mathrm{e}^{-\pi \mathrm{i}(1+\chi)}\right)\right] .
$$

Here we have used the explicit description of the boundary map in terms of the exponential function (see [Weg93, Exercise 9.E]). The unitary $\mathrm{e}^{-\pi \mathrm{i}(1+\chi)}=-\mathrm{e}^{-\pi \mathrm{i} \bar{\psi}(\tilde{\chi})}$ in $\mathcal{C}_{0}((-\varepsilon, \varepsilon))^{+} \cong \mathcal{C}\left(S^{1}\right)$ has winding number -1 , whereas $\bar{\psi}\left(\frac{\mathrm{x}-\mathrm{i}}{\mathrm{x}+\mathrm{i}}\right)$ has winding number +1 . Consequently,

$$
-\operatorname{Ind}_{\mathrm{L}, \mathrm{Z}}(\not D)=\left[\operatorname{pr}_{1}^{+} \varphi_{\not \supset}^{+}\left(\bar{\psi}\left(\frac{\mathrm{x}-\mathrm{i}}{\mathrm{x}+\mathrm{i}}\right)\right)\right]=\left[\operatorname{pr}_{1}^{+}(U)\right]=\Theta_{n}\left(\left[\varphi_{\not{D}} \circ \psi\right]\right) .
$$





\section{Secondary invariants on compact manifolds}

\subsection{Mapping positive scalar curvature to analysis}

In this section, we discuss higher secondary invariants of psc metrics on closed spin manifolds and as a sample application, we demonstrate how to derive the main result of [PS14; XY14b] using the theory we have developed in Chapter 2.

We begin with some preliminary definitions. Let $\Gamma$ be a countable discrete group and fix a model for the classifying space $\mathrm{B} \Gamma$ as a locally finite simplicial complex. Denote its universal covering by EГ. By endowing each simplex with the Euclidean metric, turn $\mathrm{B} \Gamma$ and $\mathrm{E} \Gamma$ into a locally compact geodesic metric spaces, see [BH99]. We will use this metric structure in our constructions below.

Definition 3.1.1. (1) The K-homology of $\Gamma$ is

$$
\mathrm{K}_{*}(\Gamma):=\operatorname{colim}_{\tilde{Z} \subset \mathrm{E} \Gamma} \mathrm{K}_{*}^{\Gamma}(\tilde{Z}),
$$

(2) the structure group of $\Gamma$ is

$$
\mathrm{S}_{*}(\Gamma):=\underset{\tilde{Z} \subset \mathrm{E} \Gamma}{\operatorname{colim}} \mathrm{S}_{*}^{\Gamma}(\tilde{Z})
$$

where in both cases the colimits range over $\Gamma$-invariant cocompact subsets of $\mathrm{E} \Gamma$.

If $X$ is a cocompact free $\Gamma$-space, then the $\mathrm{K}$-theory of $\mathrm{C}_{\Gamma}^{*}(X)$ is canonically isomorphic to the K-theory of the reduced group $C^{*}$-algebra $\mathrm{C}_{\mathrm{r}}^{*} \Gamma$, see [HR00, Lemma 12.5.3]. Thus Corollary 2.1.4 implies that we have a long exact sequence as follows:

$$
\cdots \rightarrow \mathrm{K}_{*+1}\left(\mathrm{C}_{\mathrm{r}}^{*} \Gamma\right) \stackrel{\partial^{\Gamma}}{\rightarrow} \mathrm{S}_{*}(\Gamma) \stackrel{q^{\Gamma}}{\rightarrow} \mathrm{K}_{*}(\Gamma) \stackrel{\alpha^{\Gamma}}{\rightarrow} \mathrm{K}_{*}\left(\mathrm{C}_{\mathrm{r}}^{*} \Gamma\right) \rightarrow \cdots
$$

Definition 3.1.2. Let $M$ be a closed $n$-dimensional spin manifold together with a continuous map $u: M \rightarrow \mathrm{B} \Gamma$. Let $g \in \mathcal{R}^{+}(M)$. Let $\bar{M} \rightarrow M$ be the $\Gamma$-covering classified by $u$ and denote the lift of $g$ to $\bar{M}$ by $\bar{g}$. The higher $\rho$-invariant of $g$ is defined as follows:

$$
\rho^{u}(g):=\bar{u}_{*} \rho^{\Gamma}(\bar{g}) \in \mathrm{S}_{n}(\Gamma),
$$

where $\rho^{\Gamma}(\bar{g}) \in \mathrm{S}_{n}^{\Gamma}(\bar{M})$ is the equivariant $\rho$-invariant of $\bar{g}$.

If $\Gamma=\pi_{1}(M)$ and $u$ the map that classifies the universal covering of $M$, then we will write $\rho^{\Gamma}(g)$ for $\rho^{u}(g)$.

If $\Gamma$ is torsion-free, then the Baum-Connes conjecture is equivalent to vanishing of $\mathrm{S}_{*}(\Gamma)$. As currently there is no known counterexample to the Baum-Connes conjecture, we need to work with groups that have torsion in order to find non-zero higher $\rho$ invariants in $\mathrm{S}_{*}(\Gamma)$. If $\Gamma$ has torsion, then injectivity of the Baum-Connes assembly 
map (or even slightly weaker assumptions) can be used to show the existence of many examples of pairs of psc metrics with different higher $\rho$-invariants, see for instance [WY13; XY13].

Definition 3.1.3. Let $W$ be a compact $n+1$-dimensional spin manifold with boundary together with a continuous map $u: W \rightarrow \mathrm{B} \Gamma$. Let $g \in \mathcal{R}^{+}(M)$. Let $\bar{W} \rightarrow W$ be the $\Gamma$-covering classified by $u$ and denote the lift of $g$ to $\bar{M}$ by $\bar{g}$. The higher APS-index of $(W, g)$ is defined as follows:

$$
\alpha_{\mathrm{APS}}^{u}(W, g):=\operatorname{Ind}_{\mathrm{APS}}^{\Gamma}(\bar{W}, \bar{M}, \bar{g}) \in \mathrm{K}_{n+1}\left(\mathrm{C}_{\Gamma}^{*}(\bar{M})\right)=\mathrm{K}_{n+1}\left(\mathrm{C}_{\mathrm{r}}^{*} \Gamma\right),
$$

where we use the coarse APS-index from Subsection 2.2.3.

Moreover, if $M$ is a closed spin manifold, we will write $\alpha^{u}(M)$ for $\alpha_{\mathrm{APS}}^{u}(M, \emptyset)=$ $\operatorname{Ind}^{\Gamma}(\bar{M})$ (where we view $M$ as a manifold with empty boundary). In this case, if $\Gamma=\pi_{1}(M)$ and $u$ is the map that classifies the universal covering of $M$, then we will write $\alpha^{\Gamma}(M)$ for $\alpha^{u}(M)$.

Similarly as in Subsection 2.2.4, we have a higher index difference:

Definition 3.1.4. Let $M$ be a closed $n$-dimensional spin manifold together with a continuous map $u: M \rightarrow \mathrm{B} \Gamma$. Let $g_{0}, g_{1} \in \mathcal{R}^{+}(M)$. Let $\bar{M} \rightarrow M$ be the $\Gamma$-covering classified by $u$ and denote the lift of $g$ to $\bar{M}$ by $\bar{g}$. The higher index difference of $g_{0}$ and $g_{1}$ is defined as follows:

$$
\alpha_{\text {diff }}^{u}\left(g_{0}, g_{1}\right)=\alpha_{\mathrm{APS}}^{u}\left(M \times[0,1], g_{0} \sqcup g_{1}\right) \in \mathrm{K}_{n+1}\left(\mathrm{C}_{\mathrm{r}}^{*} \Gamma\right) .
$$

We will briefly review the definition of $\mathrm{P}_{*}^{\text {spin }}(\mathrm{B} \Gamma)$, a bordism group due to Stolz consisting of psc metrics on spin manifolds with $\Gamma$-covering. We will also consider the group $\mathrm{R}_{*}^{\text {spin }}(\mathrm{B} \Gamma)$, which serves as a relative term for the forgetful map from of $\mathrm{P}_{*}^{\text {spin }}(\mathrm{B} \Gamma)$ to spin bordism $\Omega_{*}^{\text {spin }}(\mathrm{B} \Gamma)$. In addition, the relative group $\mathrm{R}_{n}^{\text {spin }}(\mathrm{B} \Gamma)$ contains all the information on the existence problem of psc metrics on closed spin $n$-manifolds with fundamental group $\Gamma$, and $\mathrm{R}_{n+1}^{\text {spin }}(\mathrm{B} \Gamma)$ contains all the information on the concordance classification of psc metrics on closed spin $n$-manifolds with fundamental group $\Gamma$. For more details, we refer to the survey article [RS01].

Definition 3.1.5. Given two closed spin manifolds with continuous maps $u_{i}: M_{i} \rightarrow \mathrm{B} \Gamma$, endowed with $g_{i} \in \mathcal{R}^{+}\left(M_{i}\right), i=0,1$, we say that $\left(M_{0}, u_{0}, g_{0}\right)$ and $\left(M_{1}, u_{1}, g_{1}\right)$ are bordant if there exists a compact spin manifold together with a map $v: W \rightarrow \mathrm{B} \Gamma$ and boundary $\partial W=M_{0} \sqcup\left(-M_{1}\right)$ such that there exists $h \in \mathcal{R}^{+}(W)$ with $\partial h=g_{0} \sqcup g_{1}$.

The group of such bordism classes is denoted by $\mathrm{P}_{n}^{\text {spin }}(\mathrm{B} \Gamma)$.

Definition 3.1.6. Let $W_{i}$ be a compact $(n+1)$-dimensional spin manifold with boundary together with continuous maps $u_{i}: W_{i} \rightarrow \mathrm{B} \Gamma$ and $g_{i} \in \mathcal{R}^{+}\left(\partial W_{i}\right), i=0,1$, as in Definition 3.1.3. Then we say that $\left(W_{0}, g_{0}\right)$ and $\left(W_{1}, g_{1}\right)$ are bordant if

(i) $\left(\partial W_{0}, u_{0} \uparrow \partial W_{0}, g_{0}\right)$ and $\left(\partial W_{1}, u_{1} \uparrow \partial W_{1}, g_{1}\right)$ are bordant as in Definition 3.1.5 via an $n+1$-spin manifold $Y$ with boundary $\partial Y=\partial W_{0} \sqcup\left(-\partial W_{1}\right)$ and $h \in \mathcal{R}^{+}(Y)$ with $\partial h=g_{0} \sqcup g_{1}$. 
(ii) There exists an $(n+2)$-dimensional compact spin manifold $V$ (possibly with corners of codimension up to 2) such that $\partial Z=Y$.

The group of such bordism classes is denoted by $\mathrm{R}_{n+1}^{\mathrm{spin}}(\mathrm{В} \Gamma)$.

The following statements are direct consequences of the theory developed in Chapter 2:

Corollary 3.1.7 (see Corollary 2.2.22). If $g_{0}, g_{1} \in \mathcal{R}^{+}(M)$ are concordant, then $\alpha_{\text {diff }}^{u}\left(g_{0}, g_{1}\right)=0$.

Corollary 3.1.8 (see Corollary 2.4.18). We have $\partial^{\Gamma}\left(\alpha_{\mathrm{APS}}^{u}(W, g)\right)=\rho^{u}(g)$.

Corollary 3.1.9 (see Corollary 2.4.19). We have $\partial^{\Gamma}\left(\alpha_{\text {diff }}^{u}\left(g_{0}, g_{1}\right)\right)=\rho^{u}\left(g_{0}\right)-\rho^{u}\left(g_{1}\right)$.

Corollary 3.1.10 (see Corollary 2.4.20 for $Z=\emptyset$ ). If $\left(M_{0}, u_{0}, g_{0}\right)$ and $\left(M_{1}, u_{1}, g_{1}\right)$ are bordant, then $\rho^{u_{1}}\left(g_{1}\right)=\rho^{u_{2}}\left(g_{2}\right)$.

Corollary 3.1.11. If $\left(W_{0}, g_{0}\right)$ and $\left(W_{1}, g_{1}\right)$ are bordant, then $\alpha_{\mathrm{APS}}^{u}\left(W_{0}, g_{0}\right)$ is equal to $\alpha_{\mathrm{APS}}^{u}\left(W_{1}, g_{1}\right)$.

Proof of Corollary 3.1.11. Let $Y, h$ and $V$ be as in Definition 3.1.6. For $i \in\{0,1\}$, set $\left(W_{i}\right)_{\infty}:=W_{i} \cup_{\partial W_{i}} \partial W_{i} \times \mathbb{R}_{\geqslant 0}$ and $\tilde{V}_{\infty}:=V \cup_{Y} Y \times \mathbb{R}_{\geqslant 0}$. Then $\partial \tilde{V}_{\infty}=\left(-\left(W_{0}\right)_{\infty}\right) \sqcup$ $\left(W_{1}\right)_{\infty}$. We can extend $h$ to $h_{\infty} \in \mathcal{R}_{V}^{+}\left(V_{\infty}\right)^{\Gamma}$. Let $\iota_{i}:\left(\tilde{W}_{i}\right)_{\infty} \hookrightarrow \tilde{V}_{\infty}$ be the inclusion. It follows from Corollary 2.4.20 (applied to $\left(V_{\infty}, \partial V_{\infty} / / W_{0} \sqcup W_{1}, h_{\infty}\left\lceil\partial V_{\infty}\right)\right)$ that

$$
0=\left(\iota_{0}\right)_{*} \rho_{W_{0}}^{\Gamma}\left(\left(W_{0}\right)_{\infty}\right)-\left(\iota_{1}\right)_{*} \rho_{W_{1}}^{\Gamma}\left(\left(W_{1}\right)_{\infty}\right) \in \mathcal{R}_{V}^{+}\left(V_{\infty}\right)^{\Gamma} .
$$

By definition of the APS-index (see Subsection 2.2.3), this implies that

$$
\begin{aligned}
\left(j_{0}\right)_{*} \operatorname{Ind}_{\mathrm{APS}}^{\Gamma}\left(\tilde{W}_{0}, \partial \tilde{W}_{0}, g_{0}\right)=\left(j_{1}\right)_{*} \operatorname{Ind}_{\mathrm{APS}}^{\Gamma}\left(\tilde{W}_{1}, \partial \tilde{W}_{1}, g_{1}\right) & \\
& \in \mathrm{K}_{n+1}\left(\mathrm{C}_{\Gamma}^{*}(\tilde{Y})\right) \cong \mathrm{K}_{n+1}\left(\mathrm{C}_{\mathrm{r}}^{*} \Gamma\right),
\end{aligned}
$$

where $j_{i}: \partial \tilde{W}_{i} \hookrightarrow \tilde{Y}$ are the inclusion maps. By Definition 3.1.3, this completes the proof.

Remark 3.1.12. While Corollaries 3.1.8 to 3.1 .10 only rely on the version of the delocalized APS index theorem due to Piazza-Schick [PS14] (that is, Corollary 2.4.18), the proof of Corollary 3.1.11 utilizes the full power of Corollary 2.4.20 (and hence Theorem 2.4.15) in its form for partial secondary invariants.

The groups $\mathrm{R}_{*}^{\text {spin }}(\mathrm{B} \Gamma)$ and $\mathrm{P}_{*}^{\text {spin }}(\mathrm{B} \Gamma)$ fit into a long exact sequence, Stolz' positive scalar curvature sequence, together with the spin bordism group $\Omega_{*}^{\text {spin }}(\mathrm{B} \Gamma)$. Corollaries 3.1.8, 3.1.10 and 3.1.11 imply that there exists a transformation from the Stolz sequence to the Higson-Roe sequence (3.1.1):

Theorem 3.1.13 ([PS14; XY14b]). We have a well-defined commutative diagram:

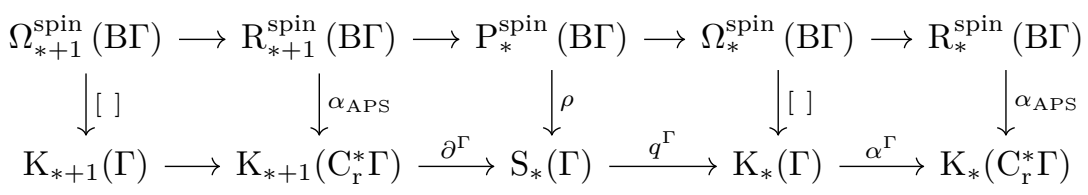




\subsection{Stability of higher secondary invariants}

As an application of Proposition 2.3.15, we prove the following results, which say that if the higher $\rho$-invariant can distinguish two psc metrics, then it can still distinguish them after taking the product with certain aspherical manifolds.

Proposition 3.2.1. Let $N$ be a closed aspherical spin q-manifold such that $\Lambda=\pi_{1}(N)$ has finite asymptotic dimension. Let $\Gamma$ be a countable discrete group. Then the map $\mathrm{S}_{*}(\Gamma) \rightarrow \mathrm{S}_{*+q}(\Gamma \times \Lambda)$ given by external product with the fundamental class $[\tilde{N}]^{\Lambda} \in \mathrm{K}_{q}^{\Lambda}(\tilde{N})$ is split-injective.

Corollary 3.2.2. Let $M_{i}$ be closed spin, $u_{i}: M_{i} \rightarrow \mathrm{B} \Gamma$ and $g_{i} \in \mathcal{R}^{+}\left(M_{i}\right), i=0,1$, such that $\rho^{u_{0}}\left(g_{0}\right) \neq \rho^{u_{1}}\left(g_{1}\right)$. Let $N$ be a closed aspherical spin q-manifold such that $\Lambda=\pi_{1}(N)$ has finite asymptotic dimension. Let $g_{N} \in \mathcal{R}(N)$ such that $g_{i} \oplus g_{N} \in$ $\mathcal{R}^{+}(M \times N)$ for $i=0,1$. Then $\rho^{u_{0} \times \operatorname{id}_{N}}\left(g_{0} \oplus g_{N}\right) \neq \rho^{u_{1} \times \operatorname{id}_{N}}\left(g_{1} \oplus g_{N}\right)$.

In particular, these assumptions imply that $\left(M_{0} \times N, u_{0} \times \mathrm{id}_{N}, g_{0} \oplus g_{N}\right)$ and $\left(M_{1} \times\right.$ $\left.N, u_{1} \times \operatorname{id}_{N}, g_{1} \oplus g_{N}\right)$ are not bordant.

Proof of Proposition 3.2.1. Since $N$ is aspherical, we may choose $\mathrm{E}(\Gamma \times \Lambda)=\mathrm{E} \Gamma \times \tilde{N}$, where $\tilde{N}$ is the universal covering of $N$. Then

$$
\mathrm{S}_{*}(\Gamma \times \Lambda)=\underset{\tilde{X} \subset \mathrm{E} \Gamma}{\operatorname{colim}} \mathrm{S}_{*}^{\Gamma \times \Lambda}(\tilde{X} \times \tilde{N}) .
$$

Since $\pi_{1}(N)$ has finite asymptotic dimension, Dranishnikov's theorem (Theorem 2.3.14) implies that $\tilde{N}$ is stably hypereuclidean. For each $\tilde{X} \subset \mathrm{E} \Gamma$, Proposition 2.3.15 gives a map $r_{\tilde{X}}: \mathrm{S}_{*+q}^{\Gamma \times \Lambda}(X \times \tilde{N}) \rightarrow \mathrm{S}_{*}^{\Gamma}(X)$ left-inverse to taking the external product with $[\tilde{N}]^{\Lambda}$. Moreover, these maps can be chosen to be natural in $\tilde{X}$ and hence define a left-inverse on the colimit. This proves the proposition.

Remark 3.2.3. Stability results concerning the index difference $\alpha_{\text {diff }}^{\Gamma}\left(g_{0}, g_{1}\right)$ analogous to Corollary 3.2.2 can be obtained in a similar fashion, for instance, by applying Proposition 2.3.15 to the partial secondary invariant $\rho_{\bar{M} \times[0,1]}^{\Gamma_{1}}(h)$ for an appropriate $h \in \mathcal{R}_{\bar{M} \times[0,1]}^{+}(\bar{M} \times \mathbb{R})$.

The manifold $N$ itself does not admit a psc metric because it is aspherical and its fundamental group has finite asymptotic dimension. In fact, if we take $N$ to be a psc manifold, then the analogue of Corollary 3.2.2 is false:

Lemma 3.2.4. Let $M$ and $N$ be closed spin manifolds which both admit psc metrics individually. Then any two product metrics which have psc on $M \times N$ are concordant.

Here we say that a Riemannian metric $h$ on $M \times N$ is a product metric if it can be written as $h=g_{M} \oplus g_{N}$ for some metrics $g_{M}, g_{N}$ on $M$, respectively $N$. 
Proof. Let $g_{M}$ and $g_{N}$ be psc metrics on $M$, respectively $N$. Denote the product metric by $h=g_{M} \oplus g_{N}$. Suppose that $\tilde{h}=\tilde{g}_{M} \oplus \tilde{g}_{N}$ is another product metric which has psc on $M \times N$. We will now show that $h$ and $\tilde{h}$ are isotopic as psc metrics. This is enough since isotopy implies concordance, see [GL80, Lemma 3]. We have the formula

$$
\operatorname{scal}_{\tilde{h}}(x, y)=\operatorname{scal}_{\tilde{g}_{M}}(x)+\operatorname{scal}_{\tilde{g}_{N}}(y)
$$

for all $x \in M, y \in N$. Thus, since $\tilde{h}$ has psc by assumption, either $\tilde{g}_{M}$ or $\tilde{g}_{N}$ has psc. We assume w.l.o.g. that it is $\tilde{g}_{M}$. By compactness we may find $\varepsilon>0$ such that $\varepsilon \tilde{g}_{M} \oplus\left(t g_{N}+(1-t) \tilde{g}_{N}\right)$ has psc for all $t \in[0,1]$. By inserting appropriate rescalings of $\tilde{g}_{M}$, this implies that $\tilde{g}_{M} \oplus \tilde{g}_{N}$ and $\tilde{g}_{M} \oplus g_{N}$ are isotopic psc metrics. Applying the same argument again, now $g_{N}$ playing the role of $\tilde{g}_{M}$, shows that $\tilde{g}_{M} \oplus g_{N}$ is isotopic to $g_{M} \oplus g_{N}$.

In particular:

Proposition 3.2.5. Let $M$ and $N$ be closed spin manifolds which both admit psc metrics individually. If $h_{0}, h_{1} \in \mathcal{R}^{+}(M \times N)$ such that $\alpha_{\mathrm{diff}}^{v}\left(h_{0}, h_{1}\right) \neq 0$ for some $v: M \times N \rightarrow \mathrm{B} \Gamma$, then at least one of $h_{0}$ and $h_{1}$ is not concordant to a product metric.

\subsection{From closed manifolds to non-compact complete manifolds}

In this section, we demonstrate how the theory we have developed so far can be applied to construct examples of complete upsc metrics on non-compact manifolds which are distinguished by certain partial secondary invariants. As input for the following constructions we will always start with psc metrics on closed manifolds which can be distinguished by the higher $\rho$-invariant. Such examples can be obtained, for example, from the methods of Weinberger-Yu [WY13] and Xie-Yu [XY13].

We start with a corollary of the secondary partitioned manifold index theorem, Theorem 2.4.6.

Corollary 3.3.1. Let $M$ be a closed spin manifold together with a map $u: M \rightarrow \mathrm{B} \Gamma$ and $g_{0}, g_{1} \in \mathcal{R}^{+}(M)$ such that $\rho^{u}\left(g_{0}\right) \neq \rho^{u}\left(g_{1}\right)$. Let $W$ be a complete spin manifold with $h_{0}, h_{1} \in \mathcal{R}^{+}(W)$ such that $W$ is partitioned by $M$ and $h_{i}$ is partitioned by $g_{i}$, $i=0,1$ (see Definition 2.4.1). Suppose that $u$ extends to a map $W \rightarrow \mathrm{B} \Gamma$. Then $h_{0}$ and $h_{1}$ are not concordant relative to $W_{-}\left(\right.$or $\left.W_{+}\right)$, where $W_{ \pm}$are the connected components of $W \backslash M$.

Proof. Let $\bar{W} \rightarrow W$ be the $\Gamma$-covering of $W$ corresponding to the map $W \rightarrow \mathrm{B} \Gamma$. Then $\bar{W}$ is partitioned by $\bar{M}$ and $\bar{h}_{i}$ by $\bar{g}_{i}$, where $\bar{M}$ is the restriction of $\bar{W}$ to $M$ and $\bar{h}_{i}$, $\bar{g}_{i}$ are the corresponding lifts of the Riemannian metrics. Theorem 2.4.6 implies that $\rho_{\bar{W}_{-}}^{\Gamma_{-}}\left(h_{0}\right) \neq \rho_{\bar{W}_{-}}^{\Gamma_{-}}\left(h_{1}\right)$ The corollary follows from this and Proposition 2.2.11.

Of course, this applies in particular to $W=M \times \mathbb{R}$. However, using our stability result about products with hypereuclidean manifolds (Proposition 2.3.15), we can generalize the product situation to higher codimensions, as will be explained in the following. 
Definition 3.3.2. Let $X, Y$ be proper metric spaces, both of which are endowed with a free and proper isometric $\Gamma$-action. We say that a $\Gamma$-equivariant coarse map $f: X \rightarrow Y$ is coarsely negligible if there exist $\Gamma$-equivariant maps $f^{\prime}: X \rightarrow X^{\prime}, f^{\prime \prime}: X^{\prime} \rightarrow Y$ such that $X^{\prime}$ is flasque and $f$ is coarsely equivalent to $f^{\prime \prime} \circ f^{\prime}$.

We say a subset $Z \subseteq Y$ is coarsely negligible in $Y$ if the inclusion map $Z \hookrightarrow Y$ is coarsely negligible. ${ }^{1}$

If $f$ is coarsely negligible, then it follows from functoriality of the Roe algebra that the map $f_{*}: \mathrm{K}_{*}\left(\mathrm{C}_{\Gamma}^{*}(X)\right) \rightarrow \mathrm{K}_{*}\left(\mathrm{C}_{\Gamma}^{*}(Y)\right)$ is zero. Moreover, if $f: Z \rightarrow Y$ is coarsely negligible, then so is $\operatorname{id}_{X} \times f: X \times Z \rightarrow X \times Y$ for any proper metric space $X$.

Example 3.3.3. If $Z \subseteq Y$ is a compact subset of a non-compact complete Riemannian manifold $Y$, then $Z$ is coarsely negligible in $Y$. To prove this, one uses that $Z$ is contained in a bounded neighborhood of some geodesic ray, compare [HPS15, Proposition 3.10]. Example 3.3.4. If $Y$ is an arbitrary proper metric space, then $Y \times[0, \infty)$ is coarsely negligible in $Y \times \mathbb{R}($ since $Y \times[0, \infty)$ is itself flasque).

Lemma 3.3.5. Let $Z \subseteq X$ be a coarsely negligible $\Gamma$-invariant subset. Then the following maps are injective:

$$
\begin{aligned}
\mathrm{K}_{*}\left(\mathrm{C}_{\Gamma}^{*}(X)\right) & \rightarrow \mathrm{K}_{*}\left(\frac{\mathrm{C}_{\Gamma}^{*}(X)}{\mathrm{C}_{\Gamma}^{*}(Z \subset X)}\right), \\
\mathrm{S}_{*}^{\Gamma}(X) & \rightarrow \mathrm{S}_{*}^{\Gamma}(X / / Z) .
\end{aligned}
$$

Proof. Since $Z$ is coarsely negligible, the map $\mathrm{K}_{*}\left(\mathrm{C}_{\Gamma}^{*}(Z \subset X)\right) \rightarrow \mathrm{K}_{*}\left(\mathrm{C}_{\Gamma}^{*}(X)\right)$ vanishes. Using the long exact sequence associated to $0 \rightarrow \mathrm{C}_{\Gamma}^{*}(Z \subset X) \rightarrow \mathrm{C}_{\Gamma}^{*}(X) \rightarrow$ $\mathrm{C}_{\Gamma}^{*}(X) / \mathrm{C}_{\Gamma}^{*}(Z \subset X) \rightarrow 0$, we conclude that the first map is injective. Moreover, the boundary map $\partial_{Z}^{\Gamma}: \mathrm{K}_{*+1}\left(\mathrm{C}_{\Gamma}^{*}(Z \subset X)\right) \rightarrow \mathrm{S}_{*}^{\Gamma}(X)$ from Corollary 2.1.4 factors through $\mathrm{K}_{*}\left(\mathrm{C}_{\Gamma}^{*}(Z \subset X)\right) \rightarrow \mathrm{K}_{*}\left(\mathrm{C}_{\Gamma}^{*}(X)\right)$ and therefore vanishes as well. Using exactness again (of the appropriate sequence from Corollary 2.1.4), we deduce injectivity of the second map.

Corollary 3.3.6. Let $M$ be a closed spin manifold together with a map $u: M \rightarrow \mathrm{B} \Gamma$ and $g_{0}, g_{1} \in \mathcal{R}^{+}(M)$. Moreover, let $Y$ be a complete spin manifold, $g_{Y} \in \mathcal{R}(Y)$ and $Z \subseteq Y$ some subset. Suppose that

(i) $\alpha_{\text {diff }}^{u}\left(g_{0}, g_{1}\right) \neq 0 \in \mathrm{K}_{n+1}\left(\mathrm{C}_{\mathrm{r}}^{*} \Gamma\right)$,

(ii) $g_{i} \oplus g_{Y} \in \mathcal{R}^{+}(M \times Y)$ for $i=0,1$,

(iii) $\left(Y, g_{Y}\right)$ is stably hypereuclidean,

(iv) $Z$ is coarsely negligible in $Y$.

Then the metrics $g_{0} \oplus g_{Y}$ and $g_{1} \oplus g_{Y}$ are not concordant on $M \times Y$ relative to $M \times Z$.

\footnotetext{
${ }^{1}$ Our notion of a coarsely negligible subset is different and more geometric than the concept of a "coarsely A-negligible subset" from [HPS15, Definition 3.9].
} 
Example 3.3.7. The theorem applies to $Y=\mathbb{R}^{q}$ and $Z=[0, \infty) \times \mathbb{R}^{q-1}$.

Proof. Suppose without loss of generality that $d_{Y}=d_{g_{Y}}$. Let $X=\bar{M}$, the covering of $M$ classified by $u$. If $g_{0} \oplus g_{Y}$ and $g_{1} \oplus g_{Y}$ were concordant on $M \times Y$ relative to $M \times Z$, then the lifted metrics $\bar{g}_{0} \oplus g_{Y}, \bar{g}_{1} \oplus g_{Y}$ would be concordant on $X \times Y$ relative to $X \times Z$. Thus, due to Corollary 2.2.22, it suffices to show that $\operatorname{Ind}_{\text {diff }}^{\Gamma}\left(\bar{g}_{0} \oplus g_{Y}, \bar{g}_{1} \oplus g_{Y} \| X \times Z\right) \neq 0$. Indeed, since $Y$ is stably hypereuclidean, Proposition 2.3.16 (applied with $Z=\emptyset$ ) shows that $\operatorname{Ind}_{\text {diff }}^{\Gamma}\left(\bar{g}_{0} \oplus g_{Y}, \bar{g}_{1} \oplus g_{Y}\right) \neq 0$. Moreover, since $Z$ is coarsely negligible in $Y$, the $\Gamma$-invariant subset $X \times Z$ is coarsely negligible in $X \times Y$. By definition, $\operatorname{Ind}_{\text {diff }}^{\Gamma}\left(\bar{g}_{0} \oplus g_{Y}, \bar{g}_{1} \oplus g_{Y}\right)$ maps to $\operatorname{Ind}_{\text {diff }}^{\Gamma}\left(\bar{g}_{0} \oplus g_{Y}, \bar{g}_{1} \oplus g_{Y}\right)$ under the map

$$
\mathrm{K}_{*}\left(\mathrm{C}_{\Gamma}^{*}(X)\right) \rightarrow \mathrm{K}_{*}\left(\frac{\mathrm{C}_{\Gamma}^{*}(X)}{\mathrm{C}_{\Gamma}^{*}(Z \subset X)}\right)
$$

The proof is complete by Lemma 3.3.5, which states that this map is injective. 



\section{Obstructions via submanifolds}

In [HPS15], Hanke-Pape-Schick have shown that, under suitable conditions, the $\alpha$-invariant of a codimension-two submanifold is an obstruction to positive scalar curvature on the ambient closed spin manifold. In this chapter, we discuss a variety of generalizations of this result: For instance, in Subsection 4.1.2 we establish a secondary codimension-two obstruction using the method of proof as in [HPS15] together with the secondary partitioned manifold index theorem (Theorem 2.4.6). Moreover, we attempt to generalize both primary and secondary obstructions to codimensions other than two. In fact, the picture is most complete for codimension one, see Subsection 4.1.1. For arbitrary codimensions, we obtain a primary result for fiber bundles over certain aspherical manifolds based on a new variant of the primary multi-partitioned manifold index theorem, see Section 4.2. The approach to the primary multi-partitioned manifold index theorem and the primary result on codimension one have been previously made available on the arXiv [Zei15]. In Section 4.3, we demonstrate that, for codimensions greater than two, there cannot exist a general secondary multi-partitioned manifold index theorem that is naively analogous to the partitioned manifold index theorem.

\subsection{Secondary obstructions via submanifolds of low codimension}

\subsubsection{Codimension one}

Theorem 4.1.1. Let $M$ be a closed spin manifold and $\Gamma=\pi_{1} M$. Let $N \subset M$ be a closed submanifold of codimension 1 with trivializable normal bundle. Suppose that the inclusion induces an injection $\pi_{1} N=: \Lambda \hookrightarrow \Gamma$. Fix a tubular neighborhood $\iota: N \times(-\varepsilon, \varepsilon) \hookrightarrow M$. There exists a commutative diagram,

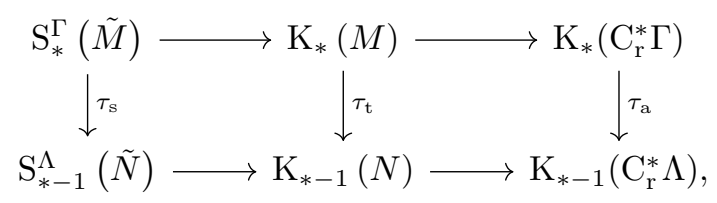

with the following properties:

(i) $\tau_{\mathrm{t}}([M])=[N]$,

(ii) $\tau_{\mathrm{a}}\left(\alpha^{\Gamma}(M)\right)=\alpha^{\Lambda}(N)$,

(iii) $\tau_{\mathrm{s}}\left(\rho^{\Gamma}(\tilde{g})\right)=\rho^{\Lambda}\left(\tilde{g}_{N}\right)$ for all $g_{M} \in \mathcal{R}^{+}(M)$ with producture structure $\iota^{*} g=g_{N} \oplus \mathrm{d} t^{2}$ on the tubular neighborhood of $N$,

(iv) $\tau_{\mathrm{a}}\left(\alpha_{\text {diff }}^{\Gamma}\left(g_{0}, g_{1}\right)\right)=\alpha_{\text {diff }}^{\Lambda}\left(g_{N, 0}, g_{N, 1}\right)$ for all $g_{0}, g_{1} \in \mathcal{R}^{+}(M)$ with product structure $\iota^{*} g_{i}=g_{N, i} \oplus \mathrm{d} t^{2}, i=0,1$, on the tubular neighborhood of $N$. 
Proof. We consider the covering $\bar{M} \rightarrow M$ such that $\pi_{1} \bar{M}=\Lambda$. With the right choice of base points it is possible to lift the inclusion $N \hookrightarrow M$ to an embedding $N \hookrightarrow \bar{M}$. Since $N \hookrightarrow \bar{M}$ has codimension one with trivial normal bundle and is an isomorphism on $\pi_{1}$, it follows that $\bar{M} \backslash N$ has precisely two connected components.

Let $\tilde{M}$ be the universal covering of $M$ and $q: \tilde{M} \rightarrow \bar{M}$ the intermediate covering. It follows that $\tilde{N}:=q^{-1}(N)$ is the universal covering of $N$ and $\tilde{N}$ is a $\Lambda$-invariant subset of $\tilde{M}$. Moroever, each component of $\tilde{M} \backslash \tilde{N}$ is $\Lambda$-invariant as well. Consider the following diagram:

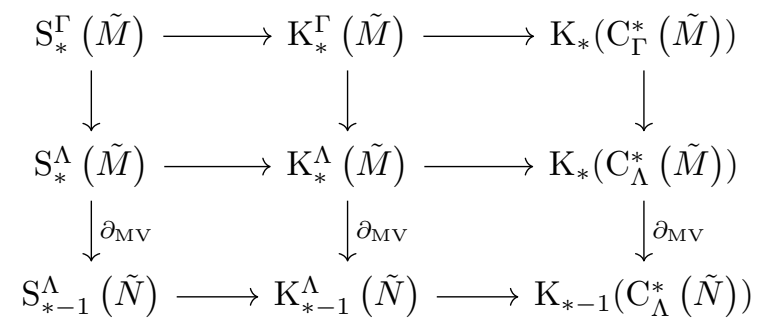

Here the top vertical arrows are the maps which forget $\Gamma$-equivariance and just remember $\Lambda$-equivariance. The bottom vertical maps are the Mayer-Vietoris boundary maps for the structure group, K-homology and Roe algebra, respectively, which are associated to the $\Lambda$-invariant cover of $\tilde{M}$ by the closures of the components of $\tilde{M} \backslash \tilde{N}$.

The maps $\tau_{x}, x \in\{\mathrm{s}, \mathrm{t}, \mathrm{a}\}$, are defined as the vertical compositions in the diagram together with the canonical identifications $\mathrm{K}_{*}^{\Gamma}(\tilde{M}) \cong \mathrm{K}_{*}(M), \mathrm{K}_{*}^{\Lambda}(\tilde{N}) \cong \mathrm{K}_{*}(N)$ and $\mathrm{K}_{*}\left(\mathrm{C}_{\Gamma}^{*}(\tilde{M})\right) \cong \mathrm{K}_{*}\left(\mathrm{C}_{\mathrm{r}}^{*} \Gamma\right), \mathrm{K}_{*}\left(\mathrm{C}_{\Lambda}^{*}(\tilde{N})\right) \cong \mathrm{K}_{*}\left(\mathrm{C}_{\mathrm{r}}^{*} \Lambda\right)$.

To prove the properties (i) to (iii), it suffices to show that $\partial_{\mathrm{MV}}[\tilde{M}]^{\Lambda}=[\tilde{N}]^{\Lambda}$ and $\partial_{\mathrm{MV}} \rho^{\Lambda}(\tilde{g})=\rho^{\Lambda}\left(\tilde{g}_{N}\right)$. Both of these statements follow from Theorem 2.4.6. For Property (iv) it suffices to show $\partial_{\mathrm{MV}} \operatorname{Ind}_{\text {diff }}^{\Lambda}\left(\tilde{g}_{0}, \tilde{g}_{1}\right)=\operatorname{Ind}_{\text {diff }}^{\Lambda}\left(\tilde{g}_{N, 0}, \tilde{g}_{N, 1}\right)$. This follows from Corollary 2.4.9.

Corollary 4.1.2. Suppose that the hypotheses of Theorem 4.1.1 hold. Then:

- If $\alpha^{\Lambda}(N) \neq 0$ in $\mathrm{K}_{*}\left(\mathrm{C}_{\mathrm{r}}^{*} \Lambda\right)$, then $\alpha^{\Gamma}(M) \neq 0$ and $M$ does not admit a metric of positive scalar curvature.

For $i \in\{0,1\}$ given $g_{i} \in \mathcal{R}^{+}(M)$ with product structure $g_{i}=g_{N, i} \oplus \mathrm{d} t^{2}, i=0,1$, near $N$, we deduce:

- If $\alpha_{\mathrm{diff}}^{\Lambda}\left(g_{N, 0}, g_{N, 1}\right) \neq 0$, then $\alpha_{\mathrm{diff}}^{\Gamma}\left(g_{M, 0}, g_{M, 1}\right) \neq 0$ and $g_{0}$ is not concordant to $g_{1}$ on $M$.

- If $\rho^{\Lambda}\left(g_{N, 0}\right) \neq \rho^{\Lambda}\left(g_{N, 1}\right)$, then $\rho^{\Gamma}\left(g_{0}\right) \neq \rho^{\Gamma}\left(g_{1}\right)$.

\subsubsection{Codimension two}

Theorem 4.1.3 below is a secondary analogue of the main result of [HPS15] involving the $\rho$-invariant. However, we have to add restrictions on the shape of the metrics near the submanifold which are not necessary for the primary theorem. 
We say that a Riemannian metric on the $\varepsilon$-disk $\mathrm{D}_{\varepsilon}$ in $\mathbb{R}^{2}$ is cylindrical near the boundary if in polar coordinates $(r, \theta) \in[0, \varepsilon] \times \mathrm{S}^{1}$ it is of the form $\mathrm{d} r^{2}+\mathrm{d} \theta^{2}$ in a neighborhood of $\{\varepsilon\} \times \mathrm{S}^{1}$.

Theorem 4.1.3. Let $M$ be a closed spin manifold and $N \subseteq M$ a closed submanifold of codimension two with trivial normal bundle. Fix a tubular neighborhood $t: N \times \mathrm{D}_{\varepsilon} \hookrightarrow M$. Suppose that the inclusion induces an injection $\Lambda:=\pi_{1} N \hookrightarrow \pi_{1} M=: \Gamma$ and a surjection $\pi_{2} N \rightarrow \pi_{2} M$. Let $g_{0}, g_{1} \in \mathcal{R}^{+}(M)$ such that $t^{*}\left(g_{i}\right)=g_{N, i} \oplus g_{\mathrm{D}, i}$, where $g_{\mathrm{D}, i}$ is cylindrical near the boundary for $i \in\{0,1\}$. If $\rho^{\Lambda}\left(g_{N, 0}\right) \neq \rho^{\Lambda}\left(g_{N, 1}\right)$ in $\mathrm{S}_{n-2}(\Lambda)$, then $g_{0}$ and $g_{1}$ are not concordant on $M$.

The following technical lemma appears in slightly more general form in [HPS15]; we exhibit this version here for the convenience of the reader.

Lemma 4.1.4 (compare [HPS15, Theorem 4.3]). Let $X$ be a connected manifold and let $N \subset X$ be a connected submanifold of codimension two with trivial normal bundle. Suppose that the inclusion $\iota: N \hookrightarrow X$ is 2-connected, that is, $\pi_{1}(\iota)$ is an isomorphism and $\pi_{2}(\iota)$ is surjective. Fix a tubular neighborhood $N \times \mathbb{R}^{2} \subset X$ and let $Y:=X \backslash\left(N \times \mathrm{B}_{1}(0)\right)$.

Then the map $\pi_{1}(i): \pi_{1}\left(N \times \mathrm{S}^{1}\right) \rightarrow \pi_{1}(Y)$ which is induced by the inclusion of the boundary $i: N \times \mathrm{S}^{1}=\partial Y \hookrightarrow Y$ is split-injective. In particular, every continuous map $\partial Y \rightarrow \mathrm{B}\left(\pi_{1}(N) \times \mathbb{Z}\right)$ extends to a continuous map $Y \rightarrow \mathrm{B}\left(\pi_{1} N \times \mathbb{Z}\right)$.

Proof. Since $\iota$ is 2-connected, it follows from the relative Hurewicz theorem that $\mathrm{H}_{k}(X, N)=0$ for $0 \leqslant k \leqslant 2$. By excision, this implies that $\mathrm{H}_{k}(Y, \partial Y)=0$ for $0 \leqslant k \leqslant 2$. In particular, we have an isomorphism $\mathrm{H}_{1}(i): \mathrm{H}_{1}\left(N \times \mathrm{S}^{1}\right) \stackrel{\cong}{\rightarrow} \mathrm{H}_{1}(Y)$. We have the following diagram,

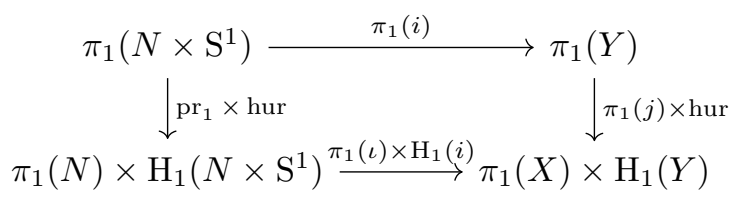

Here hur denotes the Hurewicz homomorphism. Note that $\pi_{1}\left(N \times \mathrm{S}^{1}\right)=\pi_{1}(N) \times \mathbb{Z}$ and $\mathrm{H}_{1}\left(N \times \mathrm{S}^{1}\right)=\mathrm{H}_{1}(N) \times \mathbb{Z}$; we deduce that $\mathrm{pr}_{1} \times$ hur is injective. The map $\pi_{1}(\iota) \times \mathrm{H}_{1}(i)$ is an isomorphism. Then $\left(\pi_{1}(\iota) \times \mathrm{H}_{1}(i)\right)^{-1} \circ\left(\pi_{1}(j) \times\right.$ hur $)$ maps $\pi_{1}(Y)$ onto the image of $\operatorname{pr}_{1} \times$ hur in $\pi_{1}(N) \times \mathrm{H}_{1}\left(N \times \mathrm{S}^{1}\right)$. Hence we can define

$$
r: \pi_{1}(Y) \rightarrow \pi_{1}\left(N \times \mathrm{S}^{1}\right), \quad r:=\left(\operatorname{pr}_{1} \times \operatorname{hur}\right)^{-1} \circ\left(\pi_{1}(\iota) \times \mathrm{H}_{1}(i)\right)^{-1} \circ\left(\pi_{1}(j) \times \operatorname{hur}\right)
$$

A little diagram chase implies $r \circ \pi_{1}(i)=\mathrm{id}$, which completes the proof.

Proof of Theorem 4.1.3. Let $\bar{M} \rightarrow M$ be the covering of $M$ with $\pi_{1} \bar{M}=\Lambda$. Then the inclusion $N \hookrightarrow M$ can be lifted to an embedding $\iota: N \hookrightarrow \bar{M}$. Similarly, the tubular neighborhood of $N$ lifts to $\bar{t}: N \times \mathrm{D}_{\varepsilon} \hookrightarrow \bar{M}$. By assumption, $\iota$ is a 2-equivalence. Define $Y:=\bar{M} \backslash\left(\bar{t}\left(N \times \mathrm{B}_{\varepsilon}(0)\right)\right)$. Lemma 4.1 .4 implies that the universal covering of 
$N \times \mathrm{S}^{1}=\partial Y$ extends to a $(\Lambda \times \mathbb{Z})$-covering $\tilde{Y} \rightarrow Y$ of $Y$. Note that this implies that $Y$ must be non-compact. Indeed, otherwise it would follow from Corollary 3.1.10 that $\rho^{\Lambda \times \mathbb{Z}}\left(g_{N, 0} \oplus \mathrm{d} t^{2}\right)=\rho^{\Lambda \times \mathbb{Z}}\left(g_{N, 1} \oplus \mathrm{d} t^{2}\right)=0 \in \mathrm{S}_{n-2}(\Lambda \times \mathbb{Z})$. However, Theorem 2.3.9 implies that $\rho^{\Lambda}\left(g_{N, 0}\right) \otimes[\mathbb{R}]^{\mathbb{Z}} \neq \rho^{\Lambda}\left(g_{N, 1}\right) \otimes[\mathbb{R}]^{\mathbb{Z}}$, a contradiction due to the product formula and suspension isomorphism.

Let $W=Y \cup_{\partial Y} Y$ be the double of $Y$ along its boundary and $\tilde{W}=\tilde{Y} \cup_{\partial \tilde{Y}} \tilde{Y}$ the corresponding $(\Lambda \times \mathbb{Z})$-covering. Denote by $\tilde{W}_{-}=\tilde{Y} \backslash((-\varepsilon, 0] \times \partial \tilde{Y}) \subseteq \tilde{W}$, that is, one half of the double with a collar neighborhood of the boundary removed. Then there is a Mayer-Vietoris boundary map

$$
\partial_{\mathrm{MV}}: \mathrm{S}_{n}^{\Lambda \times \mathbb{Z}}\left(\tilde{W} / / \tilde{W}_{-}\right) \rightarrow \mathrm{S}_{n-1}^{\Lambda \times \mathbb{Z}}(\tilde{N} \times \mathbb{R})
$$

associated to the cover $\tilde{W}=\tilde{Y} \cup_{\partial \tilde{Y}} \tilde{Y}$.

Let $i \in\{0,1\}$. Let $\bar{g}_{i}$ denote the restriction to $\bar{Y}$ of the lift of $g_{i}$ to $\bar{M}$. Due to the cylindrical shape of $g_{i}$ near $N \times \varepsilon \mathrm{S}^{1}$, the lift of $g_{i}$ to $\bar{M}$ restricts to a metric with product structure near $\partial Y=N \times \mathrm{S}^{1}$. Hence it can be extended to a metric $\bar{g}_{i} \in \mathcal{R}^{+}(\bar{W})$ which is partitioned by $\bar{g}_{N, i} \oplus \mathrm{d} \theta^{2} \in \mathcal{R}^{+}\left(N \times \mathrm{S}^{1}\right)$ in the sense of Definition 2.4.1. Denote its lift to $\tilde{W}$ by $\tilde{g}_{i}$. Now it follows from Theorem 2.4.6 that

$$
\partial_{\mathrm{MV}}\left(\rho_{\tilde{W}_{-}}^{\Lambda \times \mathbb{Z}}\left(\tilde{g}_{i}\right)\right)=\rho^{\Lambda}\left(\tilde{g}_{N, i}\right) \otimes[\mathbb{R}]^{\mathbb{Z}}
$$

for $i \in\{0,1\}$. We deduce that $\rho_{\tilde{W}_{-}}^{\Lambda \times \mathbb{Z}}\left(\tilde{g}_{0}\right) \neq \rho_{\tilde{W}_{-}}^{\Lambda \times \mathbb{Z}}\left(\tilde{g}_{1}\right)$.

Finally, suppose that $g_{0}$ and $g_{1}$ are concordant on $M$. Then $\bar{g}_{0}$ and $\bar{g}_{1}$ are concordant on $\tilde{W}$ relative to a bounded neighborhood of $\partial Y=N \times \mathrm{S}^{1}$ in $\bar{W}$ (we have to avoid a neighborhood of $N \times \mathrm{S}^{1}$ because we did not assume that the concordance preserves the special shape along the way). This implies that $\tilde{g}_{0}$ and $\tilde{g}_{1}$ are concordant on $\tilde{W}$ relative to a bounded neighborhood of $\partial \tilde{Y}=\tilde{N} \times \mathbb{R}$. Since any bounded neighborhood of $\partial \tilde{Y}$ is contained in a bounded neighborhood of $\tilde{W}_{-}$, we conclude that $\rho_{\tilde{W}_{-}}^{\Lambda \times \mathbb{Z}}\left(\tilde{g}_{0}\right)=\rho_{\tilde{W}_{-}}^{\Lambda \times \mathbb{Z}}\left(\tilde{g}_{1}\right)$, a contradiction.

\subsection{A multi-partitioned manifold index theorem}

In this section, we discuss the multi-partitioned manifold index theorem. Variants of this theorem have been obtained by Siegel [Sie12b] and Schick-Zadeh [SZ13] but neither provide precisely the level of generality that we need for our application to obstructions on fiber bundles (Theorem 4.2.9). Therefore, we provide a new proof in Subsection 4.2.1 below.

In the following we introduce some notation which will feature in our formulation of the multi-partitioned manifold index theorem. Let $\Gamma$ be a countable discrete group and fix a model for the classifying space $\mathrm{B} \Gamma$ as a locally finite simplicial complex. As usual, we denote its universal covering by $\mathrm{E} \Gamma$. Let $Y$ be a proper metric space. A closed subset $Z \subseteq \mathrm{B} \Gamma \times Y$ is $Y$-proper if the projection onto the second factor $\operatorname{pr}_{2} \uparrow Z: Z \rightarrow Y$ is proper. 
Definition 4.2.1. Define

$$
\begin{gathered}
\Gamma \mathrm{K}_{*}(Y):=\underset{Z}{\operatorname{colim}} \mathrm{K}_{*}(Z), \\
\Gamma \mathcal{C}_{*}(Y):=\operatorname{colim}_{Z} \mathrm{~K}_{*}\left(\mathrm{C}_{\Gamma}^{*}(\tilde{Z})\right),
\end{gathered}
$$

where the colimits range over the $Y$-proper subsets $Z \subseteq \mathrm{B} \Gamma \times Y$ and $\tilde{Z}$ denotes the lift of $Z$ to $\mathrm{E} \Gamma \times Y$.

Roughly speaking, $\Gamma \mathrm{K}_{*}(Y)$ behaves like locally finite K-homology in $Y$ and like ordinary K-homology in the "BГ-slot".

The equivariant coarse index map (compare Subsection 2.1.1) induces a map in the limit

$$
\operatorname{Ind}^{\Gamma}: \Gamma_{*}(Y) \rightarrow \Gamma \mathcal{C}_{*}(Y),
$$

which is natural in $Y$ with respect to continuous coarse maps.

Example 4.2.2. Taking $Y=*$ to be a point, $\Gamma \mathrm{K}_{*}(*)=\mathrm{K}_{*}(\Gamma)$ and $\Gamma \mathcal{C}_{*}(*) \cong \mathrm{K}_{*}\left(\mathrm{C}_{\mathrm{r}}^{*} \Gamma\right)$.

The external product in K-homology induces an external product,

$$
\Gamma \mathrm{K}_{n}(X) \otimes \mathrm{K}_{d}(Y) \stackrel{\otimes}{\rightarrow} \Gamma \mathrm{K}_{n+d}(X \times Y) .
$$

Proposition 4.2.3 (Suspension isomorphism). Let $Y$ be a proper metric space. There are isomorphisms $s$ and $\sigma$ which make the following diagram commutative,

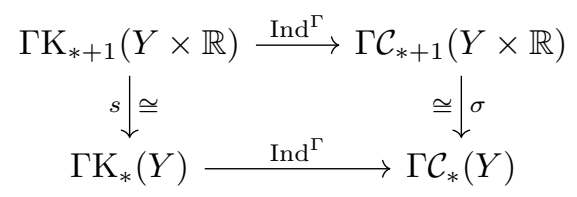

such that $s(x \otimes[\mathbb{R}])=x$ for all $x \in \Gamma \mathrm{K}_{*}(Y)$.

Proof. Let $\mathcal{Z}$ denote the directed set of all $Y \times \mathbb{R}$-proper subsets of $\mathrm{B} \Gamma \times Y \times \mathbb{R}$. Let $\mathcal{Z}_{0}$ be the collection of those $Z \in \mathcal{Z}$ such that there exists an increasing family $\left(K_{n}\right)_{n \in \mathbb{N}}$ of $Y$-proper subsets of $\mathrm{B} \Gamma \times Y$ with $Z=\bigcup_{n \in \mathbb{N}} K_{n} \times[-n, n]$. Then $\mathcal{Z}_{0}$ is a directed subset of $\mathcal{Z}$ and we will show that it satisfies the following properties:

(i) $\mathcal{Z}_{0}$ is cofinal in $\mathcal{Z}$,

(ii) For all $Z \in \mathcal{Z}_{0}$, the cover of $Z$ by $Z_{+}:=Z \cap\left(\mathrm{B} \Gamma \times Y \times \mathbb{R}_{\geqslant 0}\right)$ and $Z_{-}:=$ $Z \cap\left(\mathrm{B} \Gamma \times Y \times \mathbb{R}_{\leqslant 0}\right)$ is uniformly excisive with $Z_{0}:=Z_{+} \cap Z_{-}=Z \cap(\mathrm{B} \Gamma \times Y \times\{0\})$.

(iii) For all $Z \in \mathcal{Z}_{0}$, the $\mathrm{K}$-homology of $Z_{ \pm}$and the $\mathrm{K}$-theory of $\mathrm{C}_{\Gamma}^{*}\left(\tilde{Z}_{ \pm}\right)$vanishes.

Before verifying these properties, we show how they are used to prove the lemma: By (i), it suffices to construct isomorphisms $s_{Z}: \mathrm{K}_{*+1}(Z) \rightarrow \mathrm{K}_{*}\left(Z_{0}\right)$ and 
$\sigma_{Z}: \mathrm{K}_{*+1}\left(\mathrm{C}_{\Gamma}^{*}(\tilde{Z})\right) \rightarrow \mathrm{K}_{*}\left(\mathrm{C}_{\Gamma}^{*}\left(\tilde{Z}_{0}\right)\right)$ for each $Z \in \mathcal{Z}_{0}$ that are natural in $Z$ and so that the following diagram is commutative:

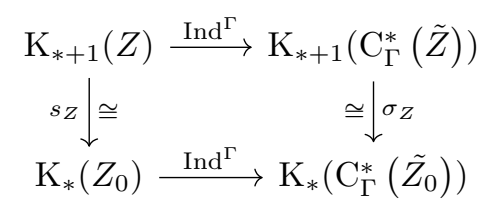

By (ii) and (iii), the respective Mayer-Vietoris boundary maps associated to the cover $Z=Z_{+} \cup_{Z_{0}} Z_{-}$do the job. The claim $s(x \otimes[\mathbb{R}])=x$ for all $x \in \Gamma K_{*}(Y)$ is then a standard fact of K-homology (see Theorem 2.3.9).

To verify (i), let $Z \in \mathcal{Z}$ be arbitrary. Then we define for each $n \in \mathbb{N}$,

$$
K_{n}:=\operatorname{pr}_{1,2}\left(Z \cap \operatorname{pr}_{3}^{-1}([-n, n])\right):=\{(b, y) \in \mathrm{B} \Gamma \times Y \mid \exists t \in[-n, n]:(b, y, t) \in Z\} .
$$

If $L \subseteq Y$ is compact, then $K_{n} \cap(\mathrm{B} \Gamma \times L) \subseteq \operatorname{pr}_{1,2}(Z \cap(\mathrm{B} \Gamma \times L \times[-n, n]))$ and the latter is compact since $Z$ is $Y \times \mathbb{R}$-proper. This shows that $K_{n}$ is $Y$-proper for all $n \in \mathbb{N}$. By construction, $Z \subseteq \bigcup_{n \in \mathbb{N}} K_{n} \times[-n, n] \in \mathcal{Z}_{0}$ and so it follows that $\mathcal{Z}_{0}$ is cofinal.

To see (ii), let $Z=\bigcup_{n \in \mathbb{N}} K_{n} \times[-n, n] \in \mathcal{Z}_{0}$ and observe that $Z_{+}=\bigcup_{n \in \mathbb{N}} K_{n} \times[0, n]$ and $Z_{-}=\bigcup_{n \in \mathbb{N}} K_{n} \times[-n, 0]$.

For (iii), we will only deal with $Z_{+}$. If $Z \in \mathcal{Z}_{0}$, then $(b, y, t) \mapsto(b, y, t+1)$ yields a well-defined map $\tau: Z_{+} \rightarrow Z_{+}$which is properly homotopic to the identity on $Z_{+}$; an Eilenberg swindle involving this map shows $\mathrm{K}_{*}\left(Z_{+}\right)=0$. Similarly, this idea also shows that $\tilde{Z}_{+}$is flasque in the sense of Definition 2.1.12 and hence $\mathrm{K}_{*}\left(\mathrm{C}_{\Gamma}^{*}\left(\tilde{Z}_{+}\right)\right)=0$.

Corollary 4.2.4. For every $\varepsilon>0$, we have

$$
\Gamma \mathrm{K}_{*}\left(\mathbb{R}^{q}\right) \cong \operatorname{colim}_{K \subset \mathrm{B} \Gamma} \mathrm{K}_{*}\left(K \times \mathbb{R}^{q}\right) \stackrel{\iota^{!}}{\cong} \operatorname{colim}_{K \subset \mathrm{B} \Gamma} \mathrm{K}_{*}\left(K \times B_{\varepsilon}(0)\right),
$$

where the colimit ranges over compact subsets $K \subseteq \mathrm{B} \Gamma$ and the second isomorphism is induced by the inclusion of the open ball $\iota: B_{\varepsilon}(0) \hookrightarrow \mathbb{R}^{q}$.

Proof. Since for a compact subset $K \subseteq \mathrm{B} \Gamma$ the set $K \times \mathbb{R}^{q}$ is $\mathbb{R}^{q}$-proper, we obtain a canonical map $J: \operatorname{colim}_{K \subset \mathrm{B} \Gamma} \mathrm{K}_{*}\left(K \times \mathbb{R}^{q}\right) \rightarrow \Gamma \mathrm{K}_{*}\left(\mathbb{R}^{q}\right)$. The $q$-fold iteration of the suspension isomorphism from Proposition 4.2.3 yields an isomorphism $s^{q}: \Gamma K_{*}\left(\mathbb{R}^{q}\right) \cong$ $\mathrm{K}_{*-q}(\mathrm{B \Gamma})$. The suspension isomorphism in $\mathrm{K}$-homology yields $t$ : $\operatorname{colim}_{K \subset \mathrm{B} \Gamma} \mathrm{K}_{*}(K \times$ $\left.\mathbb{R}^{q}\right) \cong \operatorname{colim}_{K \subset \mathrm{B} \Gamma} \mathrm{K}_{*-1}\left(K \times \mathbb{R}^{q-1}\right)$. Iteration gives $t^{q}: \operatorname{colim}_{K \subset \mathrm{B} \Gamma}\left(K \times \mathbb{R}^{q}\right) \cong \mathrm{K}_{*-q}(\mathrm{~B} \Gamma)$ such that $t^{q}=s^{q} \circ J$. In particular, this shows that $J$ must be an isomorphism.

For each $K \subseteq \mathrm{B} \Gamma$, the restriction $\iota^{!}: \mathrm{K}_{*}\left(K \times \mathbb{R}^{q}\right) \rightarrow \mathrm{K}_{*}\left(K \times B_{\varepsilon}(0)\right)$ is induced by the map on $K \times \mathbb{R}^{q}$ that is the identity on $K \times B_{\varepsilon}(0)$ and takes $K \times\left(\mathbb{R}^{q} \backslash B_{\varepsilon}(0)\right)$ to infinity in the one-point compactification of $K \times B_{\varepsilon}(0)$. Since this map induces a homotopy equivalence between the one-point compactifications, $\iota^{!}: \mathrm{K}_{*}\left(K \times \mathbb{R}^{q}\right) \rightarrow \mathrm{K}_{*}\left(K \times B_{\varepsilon}(0)\right)$ is an isomorphism. 
Corollary 4.2.4 implies that classes in $\Gamma K_{*}\left(\mathbb{R}^{q}\right)$ (and thus their images in $\Gamma \mathcal{C}_{*}\left(\mathbb{R}^{q}\right)$ ) depend only on the restrictions to arbitrarily small open subsets. A very similar localization property was exhibited by Schick-Zadeh [SZ13] and is at the heart of their approach to the multi-partitioned manifold index theorem. Analogously, our approach to the theorem in the next subsection crucially relies on Corollary 4.2.4.

\subsubsection{Multi-partitioned manifolds}

Let $f: X \rightarrow Y$ be a proper map, let $u: X \rightarrow$ B classify a covering $p: \tilde{X} \rightarrow X$. Then the image of $(u \times f): X \rightarrow \mathrm{B} \Gamma \times Y$ is $Y$-proper. Hence it induces a map

$$
(u \times f)_{*}: \mathrm{K}_{*}(X) \rightarrow \Gamma \mathrm{K}_{*}(Y) .
$$

If $f$ is also coarse, then the $\Gamma$-equivariant map $\tilde{u} \times(f \circ p): \tilde{X} \rightarrow \mathrm{E} \Gamma \times Y$ induces a map

$$
(\tilde{u} \times(f \circ p))_{*}: \mathrm{K}_{*}\left(\mathrm{C}_{\Gamma}^{*}(\tilde{X})\right) \rightarrow \Gamma \mathcal{C}_{*}(Y) .
$$

Definition 4.2.5. A complete Riemannian manifold $X$ is called $q$-multi-partitioned by a closed submanifold $M \subseteq X$ via a continuous coarse map $f: X \rightarrow \mathbb{R}^{q}$ if $f$ is smooth near $f^{-1}(0)$ such that $0 \in \mathbb{R}^{q}$ is a regular value with $f^{-1}(0)=M$.

Definition 4.2.6. Let $X$ be a complete spin $m$-manifold that is $q$-multi-partitioned by $M \subseteq X$ via $f: X \rightarrow \mathbb{R}^{q}$. Fix a $\Gamma$-covering $p: \tilde{X} \rightarrow X$ which is classified by a map $u: X \rightarrow \mathrm{B} \Gamma$. Consider the lifted map $\tilde{u}: \tilde{X} \rightarrow \mathrm{E} \Gamma$. Then we define the higher partitioned manifold index of $X$ to be

$$
\alpha_{\mathrm{PM}}^{f, u}(X):=(\tilde{u} \times(f \circ p))_{*}\left(\operatorname{Ind}^{\Gamma}(\tilde{X})\right) \in \Gamma \mathcal{C}_{m}\left(\mathbb{R}^{q}\right) .
$$

Theorem 4.2.7 (Multi-partitioned manifold index theorem). In the setup of Definition 4.2.6 we have

$$
\sigma^{q}\left(\alpha_{\mathrm{PM}}^{f, u}(X)\right)=\alpha^{\left.u\right|_{M}}(M) \in \mathrm{K}_{m-q}\left(\mathrm{C}_{\mathrm{r}}^{*} \Gamma\right),
$$

where $\sigma^{q}: \Gamma \mathcal{C}_{*}\left(\mathbb{R}^{q}\right) \rightarrow \mathrm{K}_{*-q}\left(\mathrm{C}_{\mathrm{r}}^{*} \Gamma\right)$ is the q-fold iteration of the suspension isomorphism from Proposition 4.2.3.

Proof. We have

$$
\sigma^{q}\left(\alpha_{\mathrm{PM}}^{f, u}(X)\right)=\sigma^{q}(\tilde{u} \times(f \circ p))_{*}\left(\operatorname{Ind}^{\Gamma}(\tilde{X})\right)=\operatorname{Ind}^{\Gamma}\left(s^{q}(u \times f)_{*}([X])\right) .
$$

We first deal with the product situation $X=M \times \mathbb{R}^{q}$ and $u=v \circ \mathrm{pr}_{1}$. In this special case, we have $[X]=[M] \otimes\left[\mathbb{R}^{q}\right]$, and the statement follows from (an iterated application of) the product formula from Proposition 4.2.3:

$$
\sigma^{q}\left(\alpha_{\mathrm{PM}}^{f, u}(X)\right)=\operatorname{Ind}^{\Gamma}\left(s^{q}\left(v_{*}([M]) \otimes\left[\mathbb{R}^{q}\right]\right)\right)=\operatorname{Ind}^{\Gamma}\left(v_{*}([M])\right)=\alpha^{v}(M) .
$$

In the general case, we may assume (after possibly modifying the metric near $\left.M=f^{-1}(0)\right)$ that there exists $\varepsilon>0$ such that $f^{-1}\left(B_{\varepsilon}(0)\right) \cong N \times B_{\varepsilon}(0)$ isometrically. 
Furthermore, we consider the following commutative diagram where we set $v:=\left.u\right|_{M}$ and make use of Proposition 4.2.3 and Corollary 4.2.4:

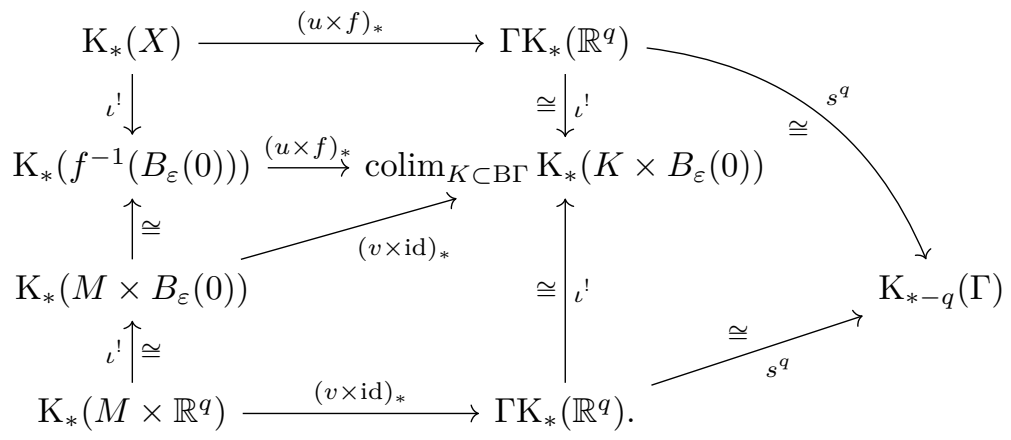

Since $f^{-1}\left(B_{\varepsilon}(0)\right) \cong M \times B_{\varepsilon}(0)$, the class $[X] \in \mathrm{K}_{m}(X)$ goes to $[M] \otimes\left[\mathbb{R}^{q}\right] \in \mathrm{K}_{m}\left(M \times \mathbb{R}^{q}\right)$ following the left vertical maps in the diagram from top to bottom. Thus the diagram implies $(u \times f)_{*}([X])=v_{*}([M]) \otimes\left[\mathbb{R}^{q}\right] \in \Gamma K_{m}\left(\mathbb{R}^{q}\right)$. This reduces the general case to the product situation which has already been established.

Corollary 4.2.8. If $\alpha^{\left.u\right|_{M}}(M) \neq 0$ in the setup of Definition 4.2.6, then $\operatorname{Ind}^{\Gamma}(\tilde{X}) \neq 0$.

\subsubsection{Fiber bundles over aspherical manifolds}

Theorem 4.2.9. Suppose that $N \stackrel{\iota}{\hookrightarrow} M \stackrel{\pi}{\rightarrow} B$ is a fiber bundle of closed spin manifolds, where $B$ is aspherical and $\pi_{1}(B)=\Gamma / \Lambda$ has finite asymptotic dimension. If $\alpha^{\Lambda}(N) \neq$ $0 \in \mathrm{K}_{n-q}\left(\mathrm{C}_{\mathrm{r}}^{*} \Lambda\right)$, then $\alpha^{\Gamma}(M) \neq 0 \in \mathrm{K}_{n}\left(\mathrm{C}_{\mathrm{r}}^{*} \Gamma\right)$. In particular, $M$ does not admit positive scalar curvature in this case.

Proof. By Theorem 2.3.14, we may assume that there exists a proper Lipschitz map $g: \tilde{B} \rightarrow \mathbb{R}^{q}$ of degree 1 (if necessary, replace the entire bundle by its product with the $k$-torus $\left.S^{1} \times \cdots \times S^{1}\right)$. Since $g$ is homotopic to a smooth map by standard approximation results [Hir94, Chapter 2], we may assume without loss of generality that $g$ is smooth. By Sard's theorem [Hir94, Chapter 3], we may further assume that 0 is a regular value. Now consider the covering $\bar{M} \rightarrow M$ with $\pi_{1}(\bar{M})=\Lambda=\pi_{1}(N)$. The bundle projection $\pi: M \rightarrow B$ lifts to a $\Gamma / \Lambda$-equivariant smooth map $\bar{\pi}: \bar{M} \rightarrow \tilde{B}$. Let $N^{\prime}:=(g \circ \bar{\pi})^{-1}(0)$. Then $\bar{M}$ is $q$-multi-partitioned by $N^{\prime}$ via $f:=g \circ \bar{\pi}$. Let $u: \bar{M} \rightarrow \mathrm{B} \Lambda$ be the map that classifies the $\Lambda$-covering $p: \tilde{M} \rightarrow \bar{M}$, where $\tilde{M}$ is the universal covering of $M$. Since $g$ has degree 1 and each fiber of $\bar{\pi}$ is a copy of $N$ inside $\bar{M}$ over each of which $p$ restricts to the universal covering, we have that $\alpha^{\left.u\right|_{N^{\prime}}}\left(N^{\prime}\right)=\alpha^{\Lambda}(N) \in \mathrm{K}_{n-q}\left(\mathrm{C}_{\mathrm{r}}^{*} \Lambda\right)$. Now consider the homomorphism $\tau: \mathrm{K}_{*}\left(\mathrm{C}_{\mathrm{r}}^{*} \Gamma\right) \rightarrow \mathrm{K}_{*-q}\left(\mathrm{C}_{\mathrm{r}}^{*} \Lambda\right)$ given by the following composition

$$
\tau: \mathrm{K}_{*}\left(\mathrm{C}_{\mathrm{r}}^{*} \Gamma\right) \cong \mathrm{K}_{*}\left(\mathrm{C}_{\Gamma}^{*}(\tilde{M})\right) \rightarrow \mathrm{K}_{*}\left(\mathrm{C}_{\Gamma}^{*}(\tilde{M})\right) \stackrel{\tilde{u} \times(f \circ p)}{\longrightarrow} \Lambda \mathcal{C}_{*}\left(\mathbb{R}^{q}\right) \stackrel{\sigma^{q}}{\longrightarrow} \mathrm{K}_{*-q}\left(\mathrm{C}_{\mathrm{r}}^{*} \Lambda\right)
$$


where the second map is induced by the inclusion $\mathrm{C}_{\Gamma}^{*}(\tilde{M}) \subseteq \mathrm{C}_{\Lambda}^{*}(\tilde{M})$ that just forgets part of the equivariance. We have

$$
\tau\left(\alpha^{\Gamma}(M)\right)=\sigma^{q}\left(\alpha_{\mathrm{PM}}^{f, u}(\bar{M})\right)=\alpha^{\left.u\right|_{N^{\prime}}}\left(N^{\prime}\right)=\alpha^{\Lambda}(N),
$$

where the first equality is by definition of $\alpha_{\mathrm{PM}}^{f, u}(\bar{M})$ and the second equality is due to Theorem 4.2.7 applied to $f=g \circ \bar{\pi}: \bar{M} \rightarrow \mathbb{R}^{q}$ and $u: \bar{M} \rightarrow \mathrm{B} \Lambda$. Since $\tau$ is a homomorphism this concludes the proof.

\subsection{The failure of secondary multi-partitioned manifold index theorems}

In view of the (primary) multi-partitioned manifold index theorem, Theorem 4.2.7, and the fact that there exists a secondary (1-)partitioned manifold index theorem, compare Theorem 2.4.6, we might also expect a secondary multi-partitioned manifold index theorem to hold. As a simple base case of such a theorem, one would predict the following localization principle for $\rho$-invariants of psc metrics on manifolds of the type $X \times \mathbb{R}^{d}:$ If $h \in \mathcal{R}^{+}\left(X \times \mathbb{R}^{d}\right)^{\Gamma}$ has product structure on a neighborhood of $X \times\{0\}$, then the iterated suspension isomorphism $\mathrm{S}_{n+d}^{\Gamma}\left(X \times \mathbb{R}^{d}\right) \cong \mathrm{S}_{n}^{\Gamma}(X)$ takes $\rho^{\Gamma}(h)$ to $\rho^{\Gamma}(h\lceil X \times\{0\})$. However, as the main result of this subsection shows, this property fails at least for all $d \geqslant 3$. As the proof of the theorem below will show, the reason for this failure of localization is that $\mathbb{R}^{d}$ for $d \geqslant 3$ can support positive scalar curvature on its own.

Theorem 4.3.1. Let $M$ be a closed manifold and $g_{0}, g_{1} \in \mathcal{R}^{+}(M)$. Let $d \in \mathbb{N}, d \geqslant 3$ and $R>0$. Then there exists a metric $h \in \mathcal{R}^{+}\left(M \times \mathbb{R}^{d}\right)$ such that

(i) the restriction of $h$ to $M \times B_{R}^{\text {eukl }}(0)$ agrees with $g_{0} \oplus g_{\mathbb{R}^{q}}$, where $g_{\mathbb{R}^{q}}$ is the Euclidean metric;

(ii) for some $S>R$, the restriction of $h$ to $M \times\left(\mathbb{R}^{d} \backslash \mathrm{B}_{S}^{\text {eukl }}(0)\right)$ agrees with $g_{1} \oplus g_{\mathbb{R}^{q}}$. Here $\mathrm{B}_{S}^{\text {eukl }}$ denotes the open ball with respect to the Euclidean metric.

Before moving on to the proof, we deduce the following corollary:

Corollary 4.3.2. Let $M$ be a closed spin manifold with $\Gamma=\pi_{1} M$ and let $g_{0}, g_{1} \in$ $\mathcal{R}^{+}(M)$ be arbitrary. Let $d \in \mathbb{N}, d \geqslant 3$ and $R>0$. Then there exists $h \in \mathcal{R}^{+}\left(M \times \mathbb{R}^{d}\right)$ such that $h\left\lceil M \times \mathrm{B}_{R}^{\text {eukl }}(0)=g_{0} \oplus g_{\mathbb{R}^{d}}\right.$ but $\operatorname{Ind}_{\text {diff }}^{\Gamma}\left(\tilde{h}, \tilde{g}_{1} \oplus g_{\mathbb{R}^{d}}\right)=0$ and $\rho^{\Gamma}(\tilde{h})=$ $\rho^{\Gamma}\left(\tilde{g}_{1}\right) \otimes\left[\mathbb{R}^{d}\right]$.

Proof. Consider $h$ as in Theorem 4.3.1. Then $\tilde{h}$ agrees with $\tilde{g}_{1} \oplus g_{\mathbb{R}^{d}}$ outside $Z:=$ $\tilde{M} \times \mathrm{B}_{S}^{\text {eukl }}(0)$. This implies that $\operatorname{Ind}_{\text {diff }}^{\Gamma}\left(\tilde{h}, \tilde{g}_{1} \oplus g_{\mathbb{R}^{d}} / / Z\right)=0$ and $\rho_{Z}^{\Gamma}(\tilde{h})=\rho_{Z}^{\Gamma}\left(\tilde{g}_{1} \oplus g_{\mathbb{R}^{d}}\right)$. But $Z$ is a coarsely negligible subset by Example 3.3.3. Therefore the result follows from Lemma 3.3.5.

By applying the corollary to $g_{0}, g_{1}$ with $\rho^{\Gamma}\left(\tilde{g}_{0}\right) \neq \rho^{\Gamma}\left(\tilde{g}_{1}\right) \in \mathrm{S}_{n}^{\Gamma}(\tilde{M})$, we derive the failure of the secondary multi-partitioned manifold index theorem that has been alluded to in the discussion before the theorem. 
Lemma 4.3.3. Let $d \geqslant 3, R>0$ and $\varepsilon>0$. Then there exist real numbers $r_{0}, r_{1}, r_{2}, S$ such that $R+1<r_{0}<r_{0}+2<r_{2}, S>R$, together with a smooth function $\varphi:(0, \infty) \rightarrow(0, \infty)$, satisfying the following conditions.

(i) The warped product metric on $(0, \infty) \times S^{d-1}$ given by $\mathrm{d} r^{2}+\varphi^{2} g_{S^{d-1}}$ has scalar curvature everywhere bounded below by $-\varepsilon$.

(ii) $\varphi(r)=r$ for all $r \leqslant R$,

(iii) $\varphi(r)=\varepsilon$ for $r \in\left[r_{0}-1, r_{0}+2\right]$,

(iv) $\varphi(r)=S+r-r_{2}$ for $r \geqslant r_{2}$.

Figure 4.1: Schematic plot of $\varphi$ from Lemma 4.3.3

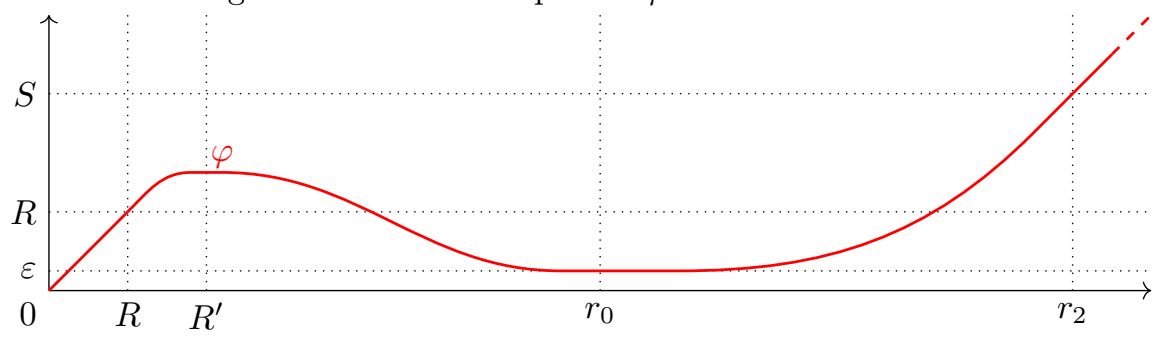

Proof. The scalar curvature of such a warped product is given by

$$
\text { scal }=-2(d-1) \frac{\varphi^{\prime \prime}}{\varphi}+(d-1)(d-2) \frac{1-\left(\varphi^{\prime}\right)^{2}}{\varphi^{2}},
$$

see for instance [Pet06, p. 69].

We begin by setting $\varphi(r):=r$ for $r \in(0, R]$. Pick any $R^{\prime}>R$ and extend $\varphi$ smoothly to the intervall $\left(0, R^{\prime}\right]$ such that $\varphi$ is constant in a neighborhood of $R^{\prime}$ and $0 \leqslant \varphi^{\prime}(r) \leqslant 1$ and $\varphi^{\prime \prime}(r) \leqslant 0$ for all $r \in\left(0, R^{\prime}\right.$. This ensures that scal $\geqslant 0$ on $\left(0, R^{\prime}\right) \times S^{d-1}$. It follows from (4.3.1) that if $\varphi$ is bounded below from zero and both $\varphi^{\prime}$ and $\varphi^{\prime \prime}$ are sufficiently small in absolute value, then scal remains positive. Thus, after picking $r_{0}$ sufficiently large, we may extend $\varphi$ smoothly to the intervall $\left(0, r_{0}+2\right]$ such that (iii) is satisfied and scal $\geqslant 0$ on $\left(0, r_{0}+2\right) \times S^{d-1}$. Finally, we extend smoothly to the intervall $\left(0, r_{2}\right]$ for some $r_{2}>r_{0}+2$ such that $\varphi$ is a linear function of slope one in a neighborhood of $r_{2}$. If $r_{2}$ is sufficiently large, this can be achieved in such a way that $\varphi \geqslant \varepsilon, 0 \leqslant \varphi^{\prime} \leqslant 1$ and $\varphi^{\prime \prime}$ is arbitarily small in absolute value on $\left(r_{0}+2, r_{2}\right)$. In particular, we can achieve scal $\geqslant-\varepsilon$. The proof is completed by extending $\varphi$ linearly on $\left(r_{2}, \infty\right)$ and, if necessary, increasing $r_{2}$ such that $S:=\varphi\left(r_{2}\right)>R$.

Proof of Theorem 4.3.1. Let $\left(g_{t}\right)_{t \in \mathbb{R}}$ be a smooth family of Riemannian metrics on $M$ such that $g_{t}=g_{0}$ for $t \leqslant 0$ and $g_{t}=g_{1}$ for $t \geqslant 1$. Let $\bar{g}$ be the resulting metric $g_{t}+\mathrm{d} t^{2}$ 
on $M \times \mathbb{R}$. Let $\varepsilon>0$ such that $-\frac{d(d-1)}{2 \varepsilon^{2}}<\operatorname{scal}(\bar{g})$ and $2 \varepsilon<\operatorname{scal}\left(g_{i}\right)$ for $i \in\{0,1\}$ and choose a function $\varphi$ as in Lemma 4.3.3.

Define a Riemannian metric on $M \times(0, \infty) \times S^{d-1}$ by

$$
\tilde{h}=g_{r-r_{0}}+\mathrm{d} r^{2}+\varphi(r)^{2} g_{S^{d-1}} .
$$

Note that $\tilde{h}$ has uniformly positive scalar curvature bounded below by $\varepsilon$ on $M \times\left(0, r_{0}\right) \times$ $S^{d-1}$ since there $\tilde{h}$ is a direct sum of the metrics $g_{0}$ on $M$ and $\mathrm{d} r^{2}+\varphi(r)^{2} g_{S^{d-1}}$ on $S^{d-1}$, the first of which has scalar curvature $>2 \varepsilon$ and the latter $>-\varepsilon$. An analogous argument applies on $M \times\left(r_{0}+1, \infty\right) \times S^{d-1}$. Finally, on $M \times\left(r_{0}, r_{0}+1\right) \times S^{d-1}$, the metric is a direct sum of $\bar{g}$ on $M \times\left(r_{0}, r_{0}+1\right)$, the scalar curvature of which is bounded below by $-\frac{d(d-1)}{2 \varepsilon^{2}}$, and the metric $\varepsilon^{2} g_{S^{d-1}}$ on $S^{d-1}$ with scalar curvature equal to $\frac{d(d-1)}{\varepsilon^{2}}$.

Let $\psi:(0, \infty) \rightarrow(0, \infty)$ be a diffeomorphism such that $\psi(r)=r$ for $r \leqslant R$ and $\psi(r)=S+r-r_{2}$ for $r \geqslant r_{2}$. Define the metric $h$ as the pushforward of $\tilde{h}$ under the diffeomorphism $M \times(0, \infty) \times S^{d-1} \rightarrow M \times\left(\mathbb{R}^{d} \backslash\{0\}\right),(x, r, \xi) \mapsto(x, \psi(r) \xi)$. 



\section{Bibliography}

[ABS64] M. F. Atiyah, R. Bott, and A. Shapiro. Clifford modules. Topology 3.suppl. 1 (1964), 3-38.

[BER14] B. Botvinnik, J. Ebert, and O. Randal-Williams. Infinite loop spaces and positive scalar curvature. 2014. arXiv: 1411.7408 [math.AT].

[BH99] M. R. Bridson and A. Haefliger. Metric spaces of non-positive curvature. Vol. 319. Grundlehren der Mathematischen Wissenschaften. Springer-Verlag, Berlin (1999), xxii+643.

[Del+11] I. Dell'Ambrogio, H. Emerson, T. Kandelaki, and R. Meyer. A functorial equivariant K-theory spectrum and an equivariant Lefschetz formula. 2011. arXiv: 1104.3441 [math.KT].

[DG15] R. J. Deeley and M. Goffeng. Realizing the analytic surgery group of Higson and Roe geometrically, part I: the geometric model. Journal of Homotopy and Related Structures (2015). DOI: 10.1007/s40062-015-0123-x.

[Dra06] A. N. Dranishnikov. On hypereuclidean manifolds. Geom. Dedicata 117 (2006), 215-231. DOI: $10.1007 / \mathrm{s} 10711-005-9025-0$.

[Dum05] D. Dumitraşcu. Asymptotic morphisms, K-homology and Dirac operators. Pacific J. Math. 218.1 (2005), 53-74. DOI: 10.2140/pjm.2005.218.53.

[GHT00] E. Guentner, N. Higson, and J. Trout. Equivariant E-theory for $C^{*}$-algebras. Mem. Amer. Math. Soc. 148.703 (2000), viii+86. DOI: 10.1090/memo/0703.

[GL80] M. Gromov and H. B. Lawson Jr. The classification of simply connected manifolds of positive scalar curvature. Ann. of Math. (2) 111.3 (1980), 423-434. DOI: 10.2307/ 1971103.

[GL83] M. Gromov and H. B. Lawson Jr. Positive scalar curvature and the Dirac operator on complete Riemannian manifolds. Inst. Hautes Études Sci. Publ. Math. 58 (1983), 83-196 (1984).

[Gro93] M. Gromov. Asymptotic invariants of infinite groups. In: Geometric group theory, Vol. 2 (Sussex, 1991). Vol. 182. London Math. Soc. Lecture Note Ser. Cambridge Univ. Press, Cambridge (1993), 1-295.

[HG04] N. Higson and E. Guentner. Group $C^{*}$-algebras and K-theory. In: Noncommutative geometry. Vol. 1831. Lecture Notes in Math. Springer, Berlin (2004), 137-251. DOI: 10.1007/978-3-540-39702-1_3.

[Hig91] N. Higson. A note on the cobordism invariance of the index. Topology 30.3 (1991), 439-443. DOI: 10.1016/0040-9383(91) 90024-X.

[Hir94] M. W. Hirsch. Differential topology. Vol. 33. Graduate Texts in Mathematics. Corrected reprint of the 1976 original. Springer-Verlag, New York (1994), x+222.

[Hit74] N. Hitchin. Harmonic spinors. Advances in Math. 14 (1974), 1-55. 
[HKT98] N. Higson, G. Kasparov, and J. Trout. A Bott periodicity theorem for infinitedimensional Euclidean space. Adv. Math. 135.1 (1998), 1-40. DOI: 10.1006/aima. 1997.1706.

[HPS15] B. Hanke, D. Pape, and T. Schick. Codimension two index obstructions to positive scalar curvature. Ann. Inst. Fourier (Grenoble) 65.6 (2015), 2681-2710.

[HR00] N. Higson and J. Roe. Analytic K-homology. Oxford Mathematical Monographs. Oxford University Press, Oxford (2000), xviii+405.

[HR05] N. Higson and J. Roe. Mapping surgery to analysis. I. Analytic signatures. KTheory 33.4 (2005), 277-299. DOI: 10.1007/s10977-005-1561-8.

[HR10] N. Higson and J. Roe. K-homology, assembly and rigidity theorems for relative eta invariants. Pure Appl. Math. Q. 6.2, Special Issue: In honor of Michael Atiyah and Isadore Singer (2010), 555-601. DOI: 10.4310/PAMQ.2010.v6.n2.a11.

[HRY93] N. Higson, J. Roe, and G. Yu. A coarse Mayer-Vietoris principle. Math. Proc. Cambridge Philos. Soc. 114.1 (1993), 85-97. DOI: 10.1017/S0305004100071425.

[HSS14] B. Hanke, T. Schick, and W. Steimle. The space of metrics of positive scalar curvature. Publ. Math. Inst. Hautes Études Sci. 120 (2014), 335-367. DOI: 10.1007/ s10240-014-0062-9.

[KW75] J. L. Kazdan and F. W. Warner. Scalar curvature and conformal deformation of Riemannian structure. J. Differ. Geom. 10 (1975), 113-134.

[Lic63] A. Lichnerowicz. Spineurs harmoniques. C. R. Acad. Sci. Paris 257 (1963), 7-9.

[LM89] H. B. Lawson Jr. and M.-L. Michelsohn. Spin geometry. Vol. 38. Princeton Mathematical Series. Princeton University Press, Princeton, NJ (1989), xii+427.

[Mur90] G. J. Murphy. $C^{*}$-algebras and operator theory. Academic Press, Inc., Boston, MA (1990), $\mathrm{x}+286$.

[Pet06] P. Petersen. Riemannian geometry. Vol. 171. Graduate Texts in Mathematics. Springer, New York (2006), xvi+401.

[PS14] P. Piazza and T. Schick. Rho-classes, index theory and Stolz' positive scalar curvature sequence. J. Topol. 7.4 (2014), 965-1004. DOI: 10.1112/jtopol/jtt048.

[QR10] Y. Qiao and J. Roe. On the localization algebra of Guoliang Yu. Forum Math. 22.4 (2010), 657-665. DOI: 10.1515/FORUM.2010.036.

[Roe03] J. Roe. Lectures on coarse geometry. Vol. 31. University Lecture Series. American Mathematical Society, Providence, RI (2003), viii+175.

[Roe16] J. Roe. Positive Curvature, Partial Vanishing Theorems and Coarse Indices. Proc. Edinburgh Math. Soc. 59 (2016), 223-233. DOI: 10.1017/S0013091514000236.

[Roe96] J. Roe. Index theory, coarse geometry, and topology of manifolds. Vol. 90. CBMS Regional Conference Series in Mathematics. Published for the Conference Board of the Mathematical Sciences, Washington, DC; by the American Mathematical Society, Providence, RI (1996), x+100. DOI: 10.1090/cbms/090.

[Ros83] J. Rosenberg. $C^{*}$-algebras, positive scalar curvature, and the Novikov conjecture. Inst. Hautes Études Sci. Publ. Math. 58 (1983), 197-212 (1984).

[RS01] J. Rosenberg and S. Stolz. Metrics of positive scalar curvature and connections with surgery. In: Surveys on surgery theory, Vol. 2. Vol. 149. Ann. of Math. Stud. Princeton Univ. Press, Princeton, NJ (2001), 353-386. 
[Sie12a] P. Siegel. Homological calculations with the analytic structure group. PhD thesis. Pennsylvania State University (2012).

[Sie12b] P. Siegel. The Mayer-Vietoris Sequence for the Analytic Structure Group. 2012. arXiv: 1212.0241 [math.KT].

[SZ13] T. Schick and M. E. Zadeh. Large scale index of multi-partitioned manifolds. 2013. arXiv: 1308.0742 [math.KT].

[Tro00] J. Trout. On graded $K$-theory, elliptic operators and the functional calculus. Illinois J. Math. 44.2 (2000), 294-309.

[Weg93] N. E. Wegge-Olsen. K-theory and $C^{*}$-algebras. A friendly approach. Oxford Science Publications. The Clarendon Press, Oxford University Press, New York (1993), xii +370 .

[Wul12] C. Wulff. Bordism invariance of the coarse index. Proc. Am. Math. Soc. 140.8 (2012), 2693-2697. DOI: 10.1090/S0002-9939-2012-11546-1.

[WY13] S. Weinberger and G. Yu. Finite part of operator K-theory for groups finitely embeddable into Hilbert space and the degree of non-rigidity of manifolds. 2013. arXiv: 1308.4744 [math.0A].

[XY13] Z. Xie and G. Yu. Higher rho invariants and the moduli space of positive scalar curvature metrics. 2013. arXiv: 1310.1136 [math.0A].

[XY14a] Z. Xie and G. Yu. A relative higher index theorem, diffeomorphisms and positive scalar curvature. Adv. Math. 250 (2014), 35-73. DOI: 10.1016/j. aim.2013.09.011.

[XY14b] Z. Xie and G. Yu. Positive scalar curvature, higher rho invariants and localization algebras. Adv. Math. 262 (2014), 823-866. DOI: 10.1016/j. aim.2014.06.001.

[Yu97] G. Yu. Localization algebras and the coarse Baum-Connes conjecture. K-Theory 11.4 (1997), 307-318.

[Zei15] R. Zeidler. Index obstructions to positive scalar curvature via submanifolds. 2015. arXiv: 1512.06781 [math.KT].

[Zei16] R. Zeidler. Positive scalar curvature and product formulas for secondary index invariants. Journal of Topology (2016). DOI: 10.1112/jtopol/jtw005.

[Zen14] V. F. Zenobi. Mapping the surgery exact sequence for topological manifolds to analysis. 2014. arXiv: 1405.6323 [math.KT]. 